\title{
Development of Improved Metrics for Predicting Brain Strain in Diverse Impacts.
}

\author{
A Dissertation \\ Presented to \\ the faculty of the School of Engineering and Applied Science \\ University of Virginia
}

\author{
in partial fulfillment \\ of the requirements for the degree \\ Doctor of Philosophy \\ by \\ Lee F. Gabler
}

December 2017 


\section{APPROVAL SHEET}

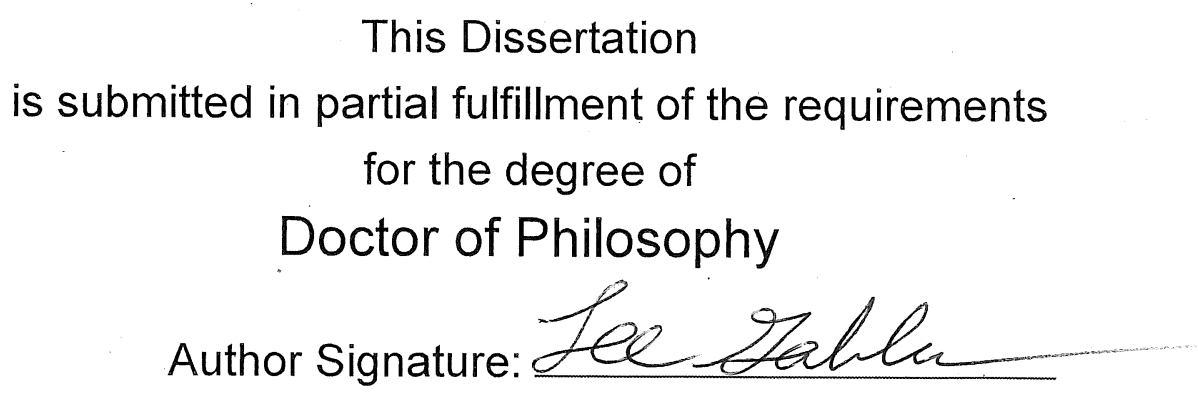

This Dissertation has been read and approved by the examining committee:

Advisor: Jeff Crandall

Committee Member: Matthew Panzer

Committee Member: Jason Kerrigan

Committee Member: Jacob Resch

Committee Member: Carlos Arregui

Committee Member:

Accepted for the School of Engineering and Applied Science:

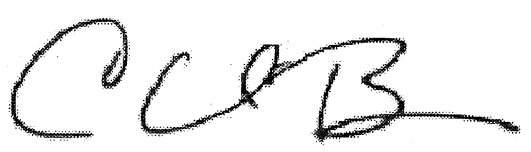

Craig $\mathrm{H}$. Benson, School of Engineering and Applied Science

December 2017 


\section{Abstract}

An estimated 3.8 million concussions are believed to occur annually in the United States, resulting in a serious concern for helmet and automotive safety manufacturers. Tissue-level deformation is believed to be the primary mechanism for concussion; however, existing dummies used in helmet and crash testing do not directly measure brain strain. Instead, brain injury assessments are made using head kinematics.

Kinematic brain injury criteria utilize a metric which relates head impact severity to a mathematical function of the velocity and/or acceleration components of translational and/or rotational head motion. Existing metrics used in helmet and crash testing are based on translational kinematics which were developed for skull fracture assessment; however, rotational motion of the head is believed to be the primary mechanism for brain strain. Although numerous rotational metrics have been proposed, many were developed using empirical methods based on limited datasets, and do not represent the mechanical principles that govern brain deformation. As a result, this renders most rotational brain injury criteria ineffective for application in a broad range of head impacts.

This dissertation presents the development of two new metrics for brain injury criteria. These metrics were developed through several steps: First, existing kinematicbased metrics were compared with finite element $(\mathrm{FE})$ model brain strains from simulations of head kinematics from football impacts and car crash tests. Correlations between brain strain and rotational metrics were highest, while translational metrics were least correlative. The Brain Injury Criterion (BrIC), an angular velocity-based metric proposed for government crashworthiness assessments had the highest overall correlation with FE 
strains; however, its performance was limited in longer duration events. Results from this study suggest that brain injury metrics use only on rotational head kinematics.

Based on the correlation analysis, a parametric study was performed using idealized head motions and an FE model to study physics of brain deformation to rotational head motion. Results from this study revealed a resonance behavior of the brain, which was adequately described using a second order system: For short duration head motions, brain deformation was proportional to maximum angular velocity, for motions of long duration brain deformation was proportional to maximum angular acceleration. For head motions near system resonance $(30-40) \mathrm{ms}$, where typical head impacts occur, brain deformations depended on both velocity and acceleration. These results explain limitations with existing rotational metrics, and were used as a basis for formulating improved metrics.

The first metric presented in this dissertation is the Universal Brain Injury Criterion (UBrIC). UBrIC was formulated based on deformation response from a second order system and uses the magnitudes of head angular velocity and acceleration to predict strainbased brain injury indicators (MPS and CSDM). Relative to existing metrics, UBrIC was a better predictor of brain strains using the football and crash test data; $\mathrm{R}^{2}=0.93$ with MPS for UBrIC vs. 0.84 for BrIC. In addition to UBrIC, a MB model analog for was developed to predict brains strains under more complicated head motions. Relative to the BrIC and UBrIC, the MB model had higher correlation with MPS overall $\left(\mathrm{R}^{2}=0.97\right)$ and performed better in nearly every impact condition assessed. This dissertation provides metrics for improved prediction of brain strains in a broad range of head impact conditions including those in football and car crashes. These metrics can be used in helmet and crash safety evaluations, and can discriminate the efficacy of improved countermeasures. 


\section{Dedication}

To Mom, Dad, Sarah, and Colden. Thank you for your love and support throughout this endeavor. 


\section{Contents}

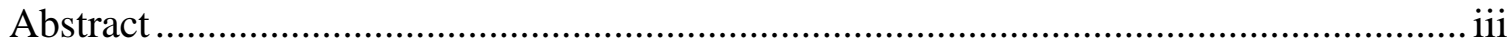

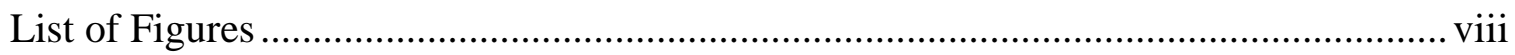

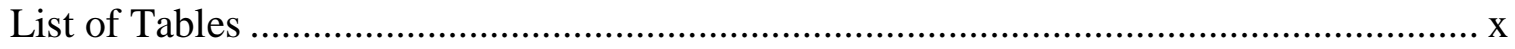

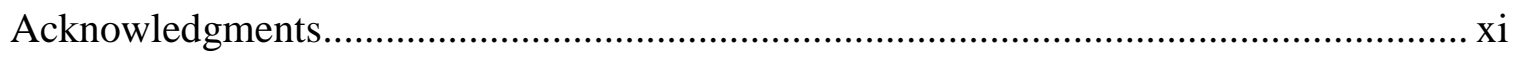

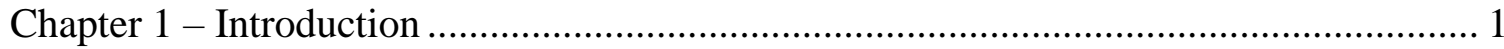

1.1 Statement of Problem ......................................................................... 1

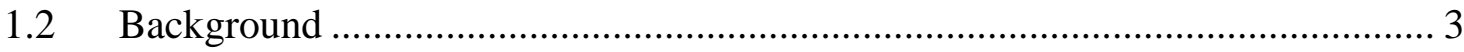

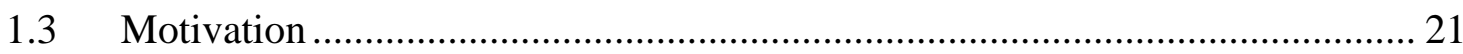

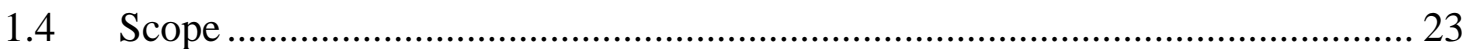

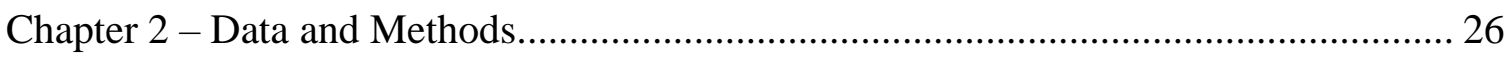

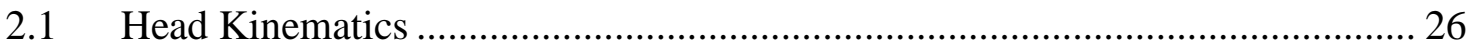

$2.2 \quad$ Finite Element Modeling ................................................................................ 33

Chapter 3 - Assessment of Kinematic-based Metrics ................................................ 38

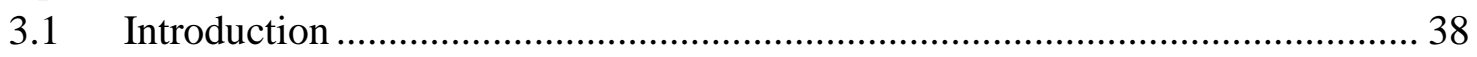

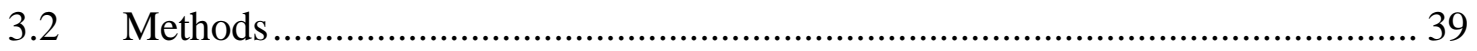

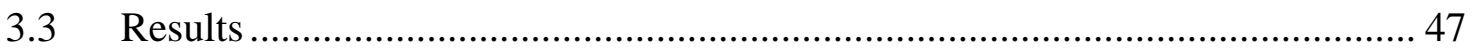

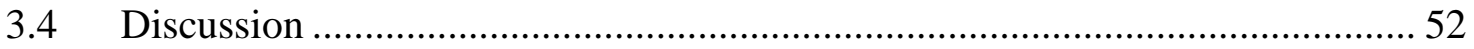

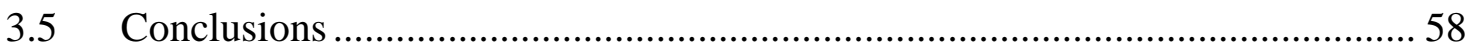

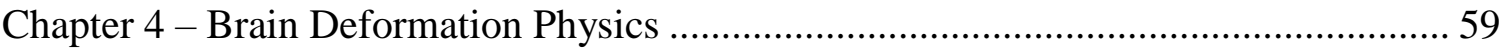

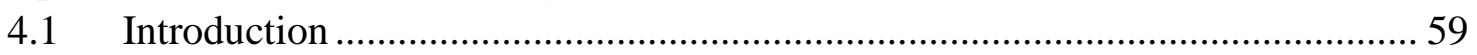

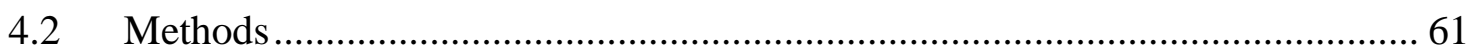

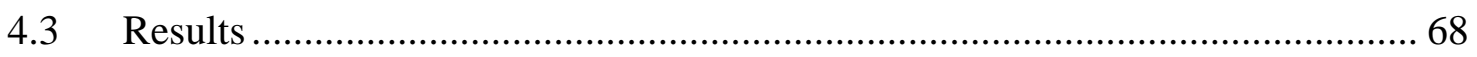

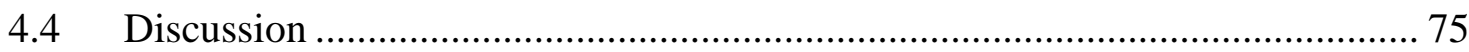

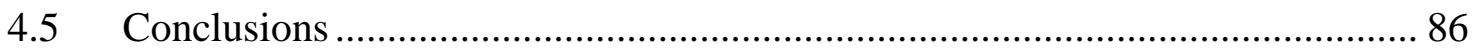

Chapter 5 - Universal Brain Injury Criterion .......................................................... 87

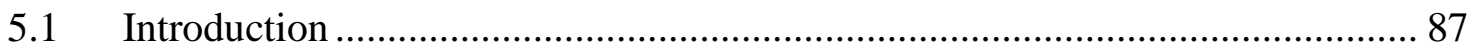

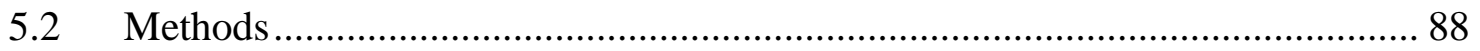

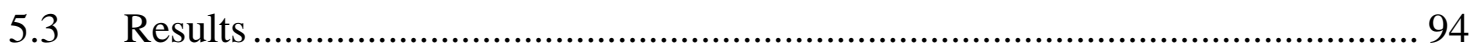

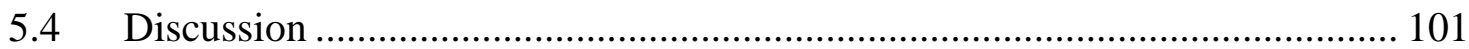

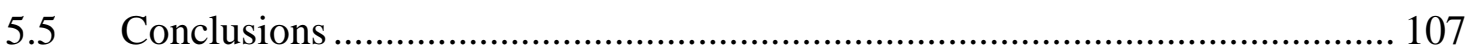

Chapter 6 - Multibody Model for the Human Brain ................................................... 108

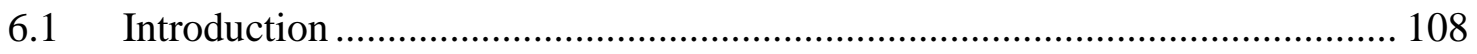

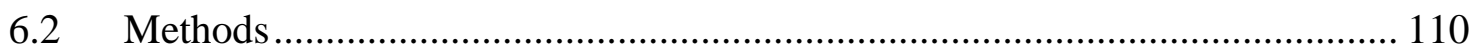

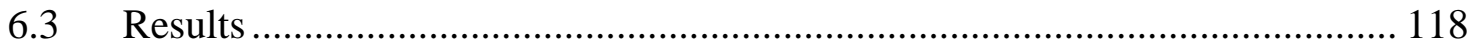

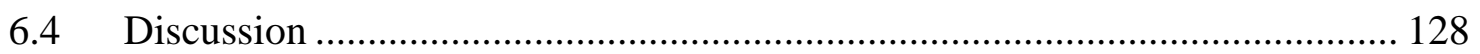

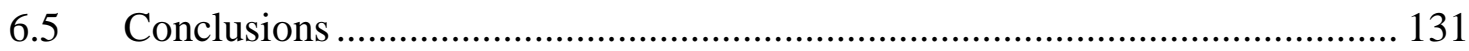

Chapter 7 - Conclusions ................................................................................... 133

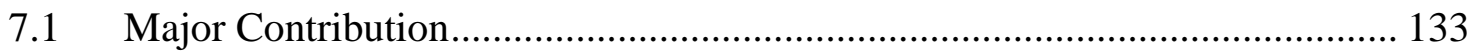




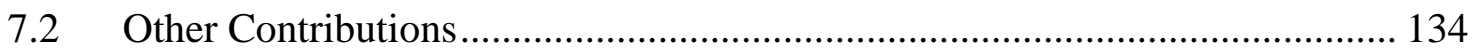

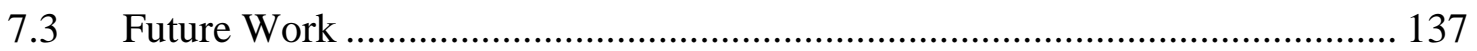

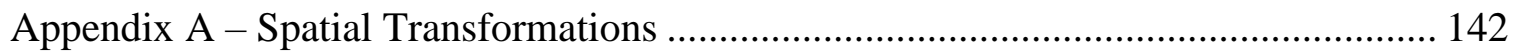

A.1 Rigid Body Kinematics ........................................................................ 142

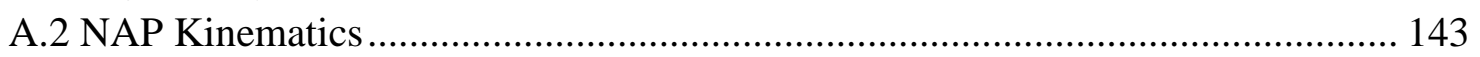

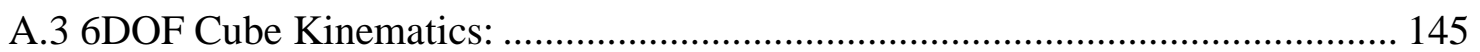

Appendix B - Scaling Head Kinematics ................................................................. 149

Appendix C - Filtering Sensitivity Study Results ...................................................... 150

Appendix D - FE Human Brain Model Specifications …………………………….... 153

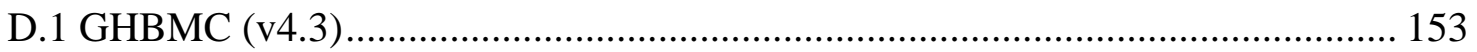

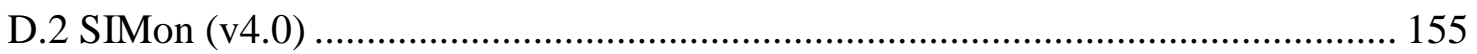

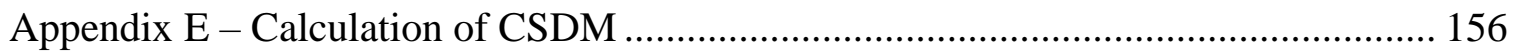

Appendix F - Solutions to the SDOF mechanical system........................................... 157

F.1 Equation of Motion of the sDOF Model ..................................................... 157

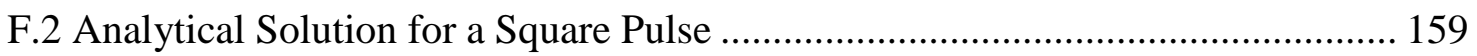

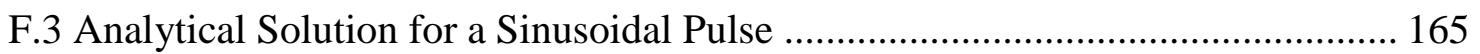

Appendix G - Solution to the mDOF mechanical system ............................................ 168

Appendix H - Supplemental Results from Chapter 5 .................................................... 169

Appendix I - List of Head Impacts from the Database ................................................ 172

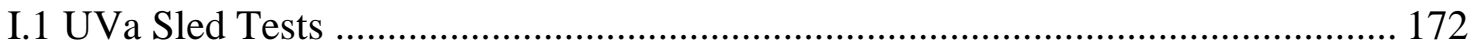

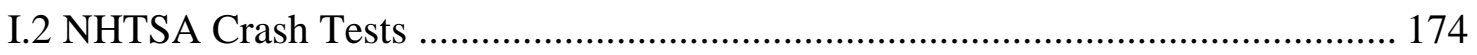

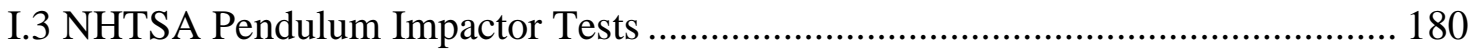

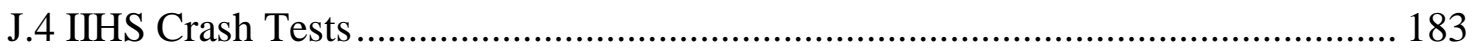

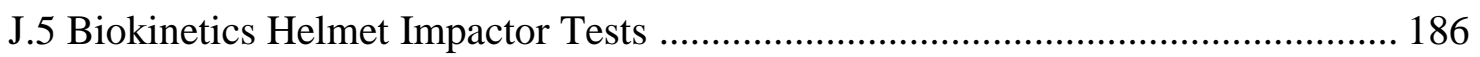

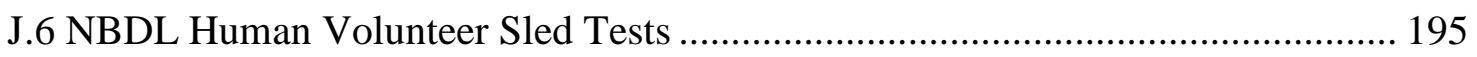

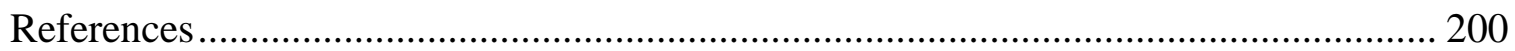

List of Published Work and Contributions ............................................................. 212 


\section{List of Figures}

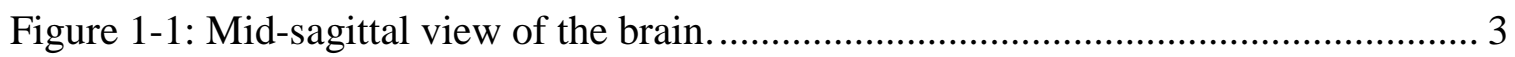

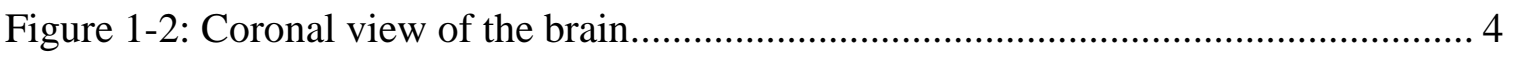

Figure 1-3: Illustrations of a neuron, and glial cell types. ............................................ 6

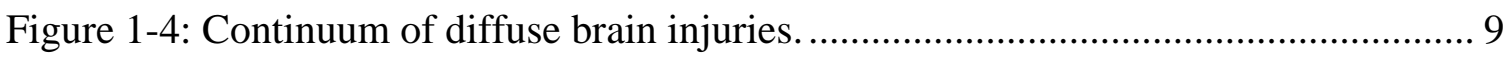

Figure 1-5: Mid Sagittal view of the GHBMC FE brain model. ...................................... 16

Figure 1-6: MB model used for the Mean Strain Criterion. ........................................... 19

Figure 1-7: Tools for modeling brain deformation mechanics....................................... 20

Figure 1-8: Approach for developing kinematic-based brain injury criteria. ................... 25

Figure 2-1: Examples of impact conditions included in this dissertation......................... 29

Figure 2-2: Work flow for processing head kinematics data.......................................... 31

Figure 2-3: Distribution of head kinematic parameters from the database....................... 32

Figure 2-4: Detailed descriptions of the GHBMC and SIMon FE brain models.............. 34

Figure 2-5: Maximum principal brain strains for several example head impacts............. 35

Figure 2-6: Description of MPS and CSDM calculations. ........................................... 36

Figure 2-7: Distribution of strain-based metrics for GHBMC and SIMon....................... 37

Figure 3-1: Kinematic-to-strain metric correlations for GHBMC and SIMon $(n=660) .48$

Figure 3-2: Scatter plots showing correlations between strain-based metrics from GHBMC

and several kinematics-based metrics.......................................................................... 49

Figure 3-3: Correlations between kinematic and strain-based metrics for various loading

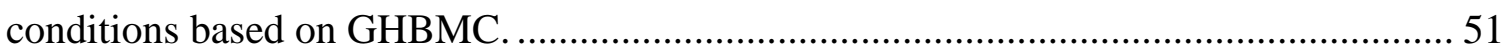

Figure 3-4: Brain strain sensitivity to translation-only, rotation-only, and combined impact

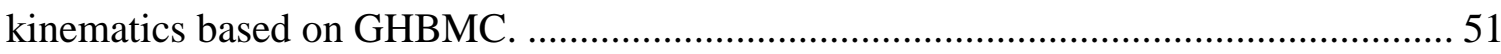

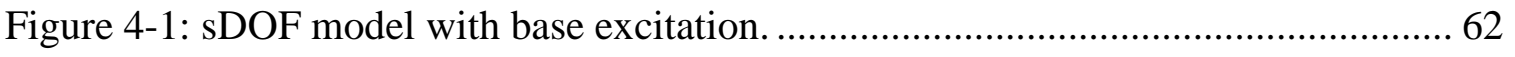

Figure 4-2: Idealized kinematic pulse applied to the FE and sDOF models................... 63

Figure 4-3: Design space for simulations used in uniaxial the parametric study. ............ 64

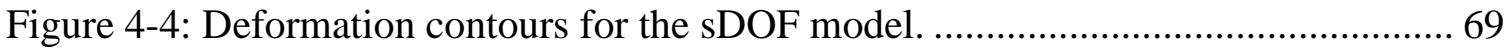

Figure 4-5: MPS contours GHBMC and SIMon. ……….......................................... 71

Figure 4-6: Sensitivity of strain-based metrics to rotational motions about $\pm y$ axis. ...... 72

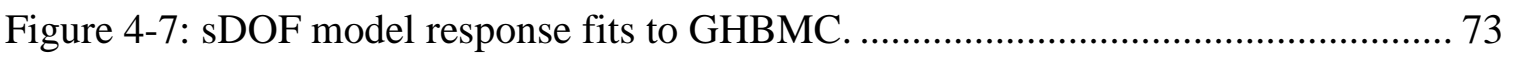

Figure 4-8: Comparison between fitted sDOF model contours and FE MPS contours... 73

Figure 4-9: Correlations between existing rotational-based metrics and the sDOF model,

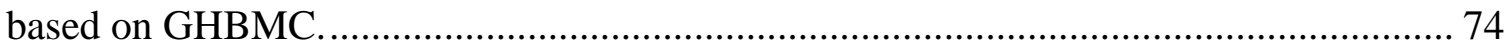

Figure 4-10: Contour plots for existing kinematic-based metrics. ................................... 75

Figure 4-11: Experimental human brain injury tolerances............................................ 79 
Figure 5-1: Deformation contours from the sDOF model used to develop UBrIC. 89

Figure 5-2: Correlations and metric-accuracy for kinematic-to-strain metric fits from GHBMC using 1595 head impacts.

Figure 5-3: Scatter plots for top performing fitted metrics to MPS and CSDM from GHBMC using 1595 head impacts. 96

Figure 5-4: Performance of top performing fitted metrics and BrIC assessed by impact condition based on GHBMC. 99

Figure 5-5: Comparison of MPS contours predicted by top performing kinematic-based metrics relative to MPS from GHBMC. 100

Figure 5-6: Performance of top performing fitted metrics and BrIC assessed for correlation and metric accuracy using NHTSA's OMDB and full frontal crash tests. 102

Figure 6-1: MB model analog for maximum brain deformation. 111

Figure 6-2: Method for choosing pulse parameters for the multiaxial parametric study.115 Figure 6-3: Comparison of FE model MPS from the biaxial loading cases. 121 Figure 6-4: Comparison of FE model MPS from the biaxial loading cases (fitted).... 123 Figure 6-5: Scatter plots showing the prediction of MPS based on the metrics developed in this dissertation using the database $(n=1747)$. 125 Figure 6-6: Performance of MB models and kinematic-based metrics assessed by impact condition based on GHBMC.

Figure 6-7: Comparison of uncoupled and coupled MB models assessed by impact condition based on metric accuracy for the res-max and max-res MB models. 128 Figure A-1: Rigid body under arbitrary three dimensional motion. 142

Figure A-2: NAP configuration. 143

Figure C-1: MPS and CSDM sensitivity to low pass filter frequency........................ 152

Figure C-2: Percent error in strain-based metrics at CFC 60 relative to CFC $1000 \ldots . . .152$

Figure F-1: sDOF mechanical system and free body diagram. ............................... 157

Figure F-2: Unit step acceleration pulse.

Figure F-3: Contours of maximum deformation for the damped and undamped sDOF model subjected to a broad range of square pulses.

Figure F-4: Half and Full cycle sinusoid acceleration pulses. 165

Figure H-1: Performance of fitted metrics assessed by impact condition based on MPS $\left(50^{\text {th }}\right.$ percentile) from GHBMC.

Figure H-2: Performance of fitted metrics assessed by impact condition based on CSDM (25\% MPS threshold) from GHBMC. 


\section{List of Tables}

Table 2-1: Summary of the impact conditions included in this dissertation..................... 30

Table 3-1: Summary of the impact conditions used in Chapter 3................................. 40

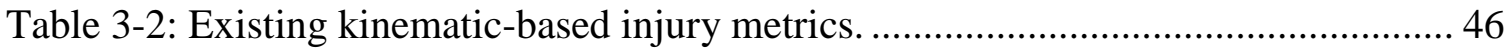

Table 3-3: Overall metric ranking by $\mathrm{R}^{2}$ based on 660 head impacts. ........................... 52

Table 3-4: Regression results using 660 head impacts and GHBMC............................... 54

Table 4-1: Range of parametric simulations used for sDOF model fits. ......................... 67

Table 4-2: Fitted parameters for the uniaxial sDOF models........................................ 74

Table 4-3: Regression results for 660 head impacts (Figure 4-9) ................................. 76

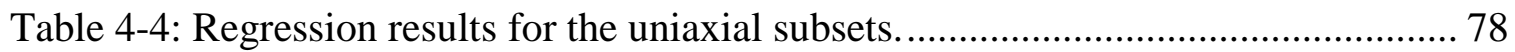

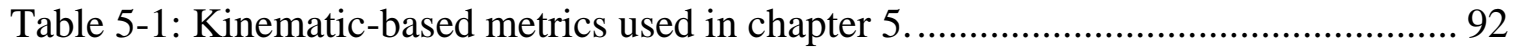

Table 5-2: Critical values for top performing kinematic metrics $(\mathrm{n}=1595) \ldots \ldots \ldots \ldots \ldots \ldots . . . . . . .97$

Table 6-1: Simulations for the multiaxial parametric study ..................................... 116

Table 6-2: Unique head motion combinations for the multiaxial parametric study...... 117

Table 6-3: Results for the proportional damping fits to the uniaxial data..................... 119

Table 6-4: Coupling conditions for the GHBMC model .......................................... 120

Table 6-5: Coupling coefficients from the regression analysis using GHBMC............ 123

Table 6-6: MB specifications and performance with the database $(n=1747) \ldots \ldots \ldots \ldots . . . . .126$

Table 7-1. Summary of the metrics developed in this dissertation............................... 134

Table A-1: Dimensions for NAP arm lengths and seismic standoffs. .......................... 148

Table C-1: Tests used in the filtering sensitivity study........................................... 150

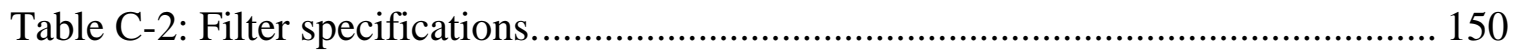

Table D-1: Mesh, mass, and density properties for GHBMC ..................................... 153

Table D-2: Material property definitions for GHBMC ............................................... 154

Table D-3: Contact and boundary definitions for GHBMC. ...................................... 154

Table D-4: Mesh, mass, and density properties for SIMon. ....................................... 155

Table D-5: Material property definitions for SIMon. ............................................... 155

Table D-6: Contact and boundary definitions for SIMon....................................... 155

Table E-1: FE model brain volume specifications for CSDM calculation.................... 156 


\section{Acknowledgments}

First, I would to thank my advisors Drs. Matthew Panzer and Jeff Crandall for their guidance and support during the course of completing this dissertation. It is a privilege to have worked with some of the greatest minds in the field, and I'm forever grateful for the opportunities they have given me. I would also like to thank my dissertation committee members: Drs. Jason Kerrigan, Jacob Resch, and Carlos Arregui for their time and effort serving on my committee; their expertise has been invaluable.

There are several other people that I would like to acknowledge for their support during my time as a graduate student. First, I would like to thank Dr. Robert Salzar. Words cannot express how grateful I am for his friendship, advice, and the opportunities he has given me. I would also like to thank my fellow graduate students Annie Bailey-Good, John Christopher, Brandon Perry, and Daniel Perez. Their friendship and support throughout graduate school has been immense, and it has been a pleasure being part of their lives. I would also like thank Thomas Adam and Mike Smayda for their friendship and support outside of graduate school. Our common interest in running made my transition to Charlottesville much easier.

Finally, I would like to thank the members of the CAB. Having a good relationship with the people you work around is as important as the work you are doing, and I feel that I've "lucked out" on that one. 


\section{Chapter 1 - Introduction}

\subsection{Statement of Problem}

Remarkable strides have been made in reducing the number of serious and fatal injuries occurring in the United States (US). Although several factors have played a role in this success, a key contributor has been the ability to leverage an improved understanding of human injury biomechanics combined with regulatory compliances to develop effective safety countermeasures and protective equipment. For example, thanks to the mandatory and voluntary requirements for automobile crashworthiness, fatality rates in motor vehicle collisions (MVC) have dropped by more than half since 1980 (NHTSA, 2014), while injury-related fatalities that were once occurring in sport and military training exercises have been substantially reduced due to use of certified personal protective equipment (Kucera et al., 2013; Pascrell, 2009).

Despite these remarkable accomplishments, TBI remains a serious global health problem (Taylor, 2017). According to the World Health Organization, TBI is expected to become the third leading cause of death by 2020 (Meaney et al., 2014). In the US, an estimated 2.8 million hospital visits resulting in TBI diagnosis occurred in 2013; an increase of almost $90 \%$ from 2001, and nearly $16 \%$ of all injury-related hospitalizations, and one-third of injury-related deaths include TBI as either a primary or secondary diagnosis (Taylor, 2017). When analyzed by principal injury mechanism, falls and struck by/against were found to be the leading causes; approximately $47 \%$ and $15 \%$, respectively of all cases each year. While MVCs were the third highest source of TBI (14\%), they were found to be the leading cause of TBI-related death among people age 5-24 years. 
The vast majority of TBI cases ( 90\%) result from closed-head impacts, i.e., without skull fracture (Santiago et al., 2012), and span a spectrum of mild-to-severe types including diffuse: concussion and diffuse axonal injury (DAI), and focal injuries: contusion, laceration, and hemorrhage (Gennarelli et al., 1985). However, the figures mentioned above represent only the civilian population, and do not account for those receiving care at a federal facility, e.g., service members, nor do they include the alarming number of unreported cases that occur annually in contact sports (Langlois et al., 2006; McCrea et al., 2004) and military training exercises (Tanielian et al., 2008). While the true burden of TBI is unknown, epidemiologists estimate that undocumented cases account for around half the total number of cases that occur annually in the US (Coronado et al., 2012).

While the mandatory and voluntary requirements for automobile countermeasures and head protective equipment have had led to a significant reduction in the number fatal injuries and skull fractures, the number of survivable TBIs spanning mild to severe are believed to be rising (Daneshvar et al., 2011; Takhounts et al., 2013; Taylor, 2017). This increase may be a result of several competing factors: clinical diagnosis of TBI has improved; the definition of milder forms of TBI such as concussion has broadened to include more symptoms; more people are surviving with milder injuries from impacts that were once fatal; and/or a heighted awareness of the potentially devastating, long term consequences associated with the disease has led to more attention resulting in a higher frequency of hospital visits and diagnoses (Taylor, 2017). Regardless, these studies show an alarming trend, and necessitate the development of improved safety countermeasures and head protective equipment that are more effective at preventing TBI. 


\subsection{Background}

This section provides background research for the content developed in this dissertation. Topics include a review of human brain anatomy and composition, brain material properties; injury mechanisms; and injury criteria, in addition to computational approaches for modeling brain mechanics during head impact. Each subsection contributes to the understanding of brain injury biomechanics in closed-head impact scenarios.

\subsubsection{Human Brain Anatomy}

The brain is the primary organ of the nervous system and serves as the control center for all bodily functions; from neuromuscular control to cognition and emotion. An adult human brain weighs, on average, $1.5 \mathrm{~kg}$ (3.3 lbs.), and is comprised of (75-80) \% water (Azevedo et al., 2009). The brain is located within the cranial cavity, and is protected by the skull; a hard structure comprised of cortical and trabecular bone layers (Figure 1-1).

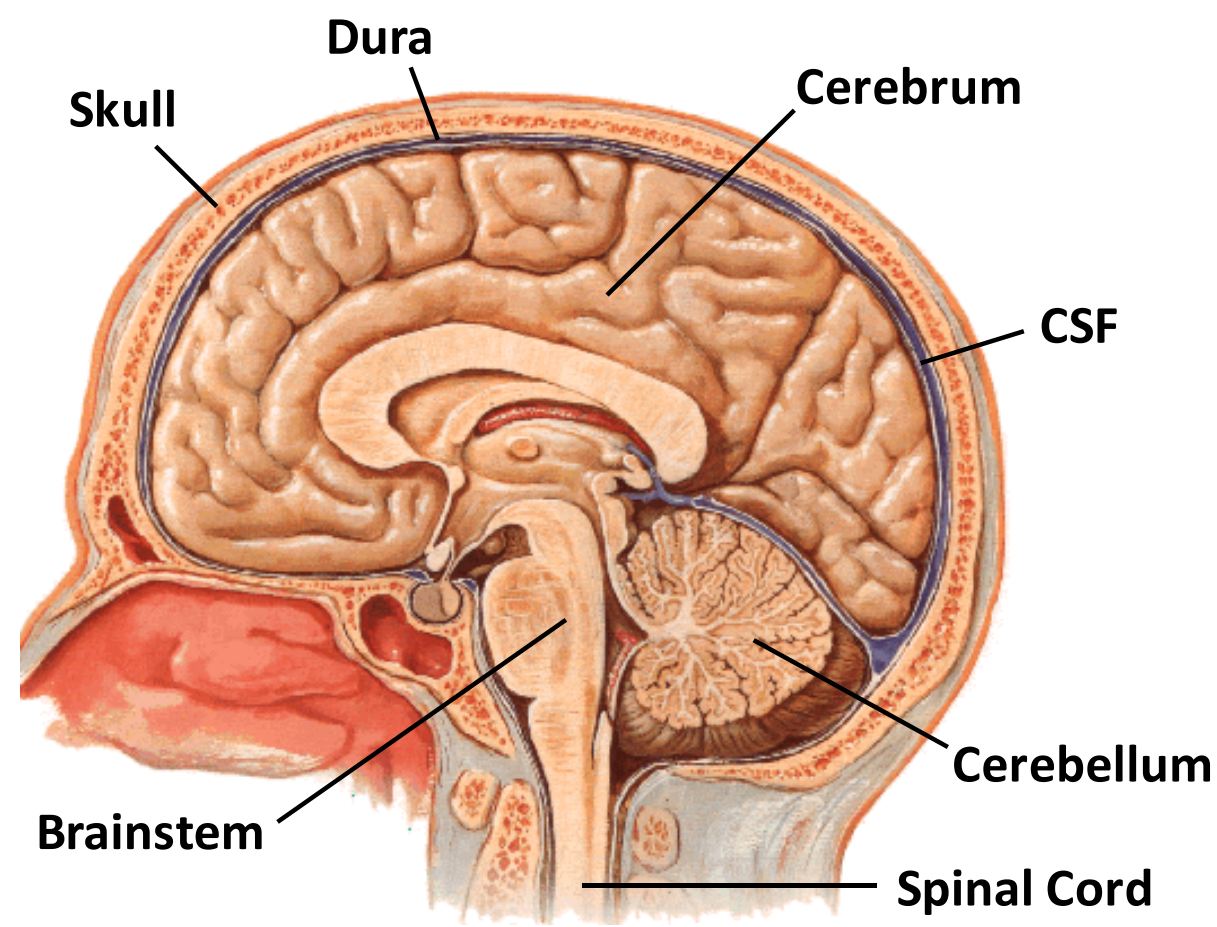

Figure 1-1: Illustration of a mid-sagittal view of the brain (Netter). 
The space between the brain and skull is occupied by cerebrospinal fluid (CSF), blood vessels, and several layers of membranes called the meninges. The dura mater (dura) is the outermost layer of the meninges, and attaches to the inner cortical layer of the skull. The brain consists of numerous structures with varying composition and function; the three largest being the cerebrum, the cerebellum, and the brainstem (Sekhar et al., 2012).

The cerebrum is the largest structure of the brain, and is located anteriorly in the cranial cavity and superior to the cerebellum and brainstem. It is responsible for processing sensory information and initiating motor function, in addition to conducting higher order thought processes including memory, learning, reasoning, and executive function (Sekhar et al., 2012). The surface of the cerebrum is convoluted and characterized by ridges (gyri) and valleys (sulci) that extend the area of the cerebral cortex, the outermost layer (Figure 1-2). The cortex is separated into four regions (lobes) based on their functions; the frontal lobe, the parietal lobe, the occipital lobe, and the temporal lobe.

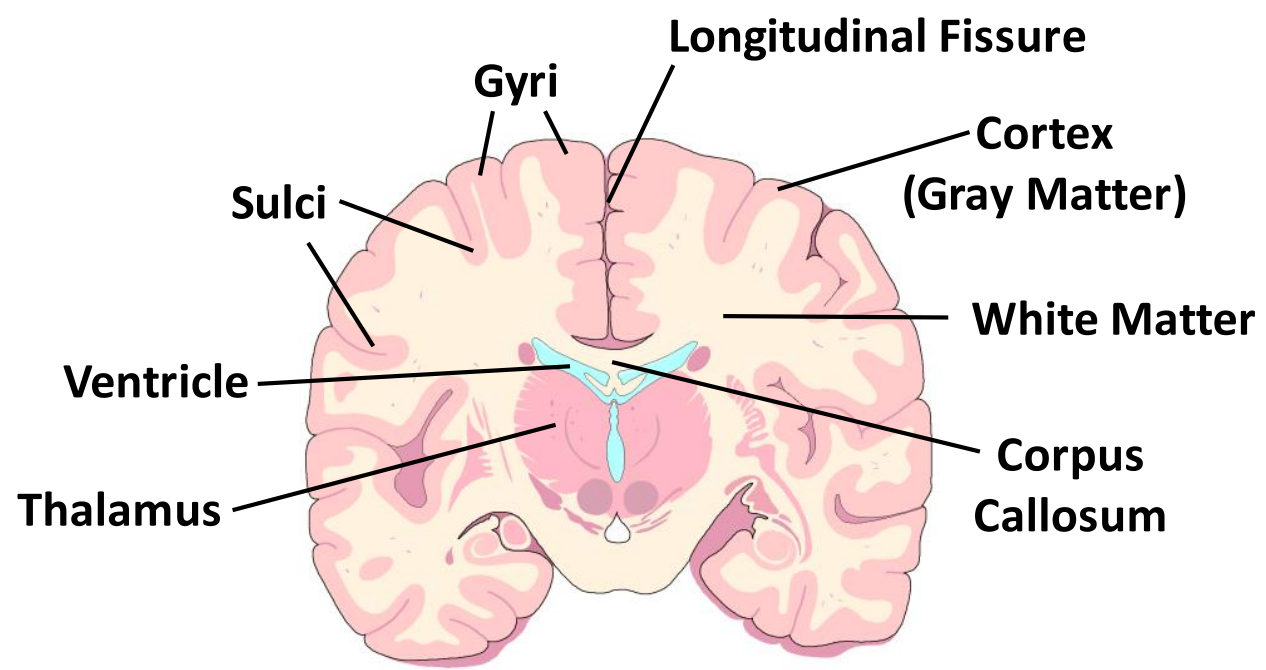

Figure 1-2: Illustration of a coronal view of the brain (LifeART).

The longitudinal fissure extends along the mid sagittal plane from the dura to the corpus callosum, and divides the cerebrum into left and right hemispheres. This space is 
occupied by CSF, and a stiff membrane that attaches to the dura called the falx cerebri (falx). The corpus callosum is a thick dense band of commissural fibers that connect the right and left hemispheres of the brain at the base of the longitudinal fissure, and transmits signals between hemispheres (Sekhar et al., 2012). Within the cerebrum there are four interconnected cavities called ventricles that supply blood to the brain. Other deep structures within the brain include the basal ganglia, thalamus, and hypothalamus, each responsible for relaying information from the cerebral cortex to the brainstem.

The cerebellum, meaning "little brain" in Latin, is located posteriorly in the cranial cavity inferior to the cerebrum, and attaches directly to the brainstem through the cerebellar peduncle. It functions to relay and coordinate motor signals between the cerebrum and body (Sekhar et al., 2012). The surface of the cerebellum differs from that of the cerebrum, and is characterized by small grooves that run parallel along the cerebellar cortex; however, there are still exist a number fissures that divide the cerebellum into different regions much like the cortex. The space between the cerebellum and cortex is filled with CSF and another membrane that connects to the dura called the tentorium. There are three lobes within the cerebellum: the anterior lobe, the posterior lobe, and the flocculonodular lobe.

The brainstem is the connection between the brain and spinal cord, and is located inferiorly to both the cerebrum and cerebellum. It serves as a conduit for passing electrical signals between the brain and body, and controls various vital biological processes including cardiac and respiratory functions as well as eating and sleeping (Sekhar et al., 2012). The brainstem consists of several substructures including the midbrain, the pons, and the medulla oblongata. The midbrain is the superior most substructure and connects 
the brainstem and cerebrum. The pons is inferior to the midbrain and connects with medulla oblongata, which transitions into the spinal cord.

Neural tissue or brain parenchyma is comprised of roughly equal numbers (120 billion) of two cell types: neurons and neuroglia (Azevedo et al., 2009). Neurons consist of a cell body and several neuronal processes called axons and dendrites, which transmit and receive electrochemical signals, respectively (Figure 1-3, A). Neuroglia (glial cells) consist of numerous different types and are believed to play a protective and supporting role in the function of the neurons (Figure 1-3, B and C). Neurons and neuroglia can be found in different proportions in different brain structures (Azevedo et al., 2009).

(A)

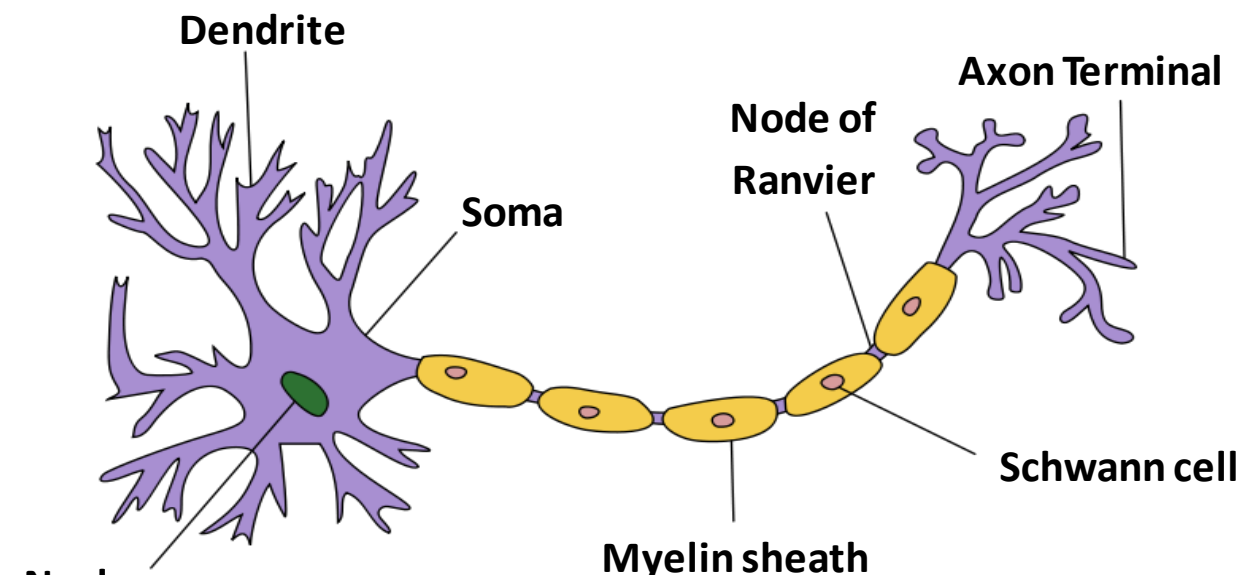

Nucleus

(B)

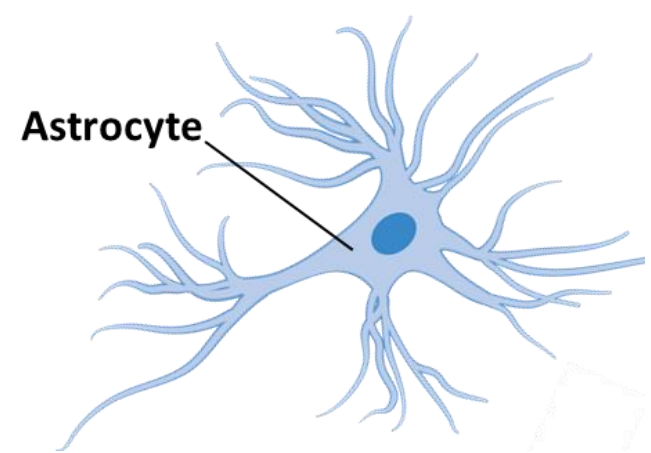

(C)

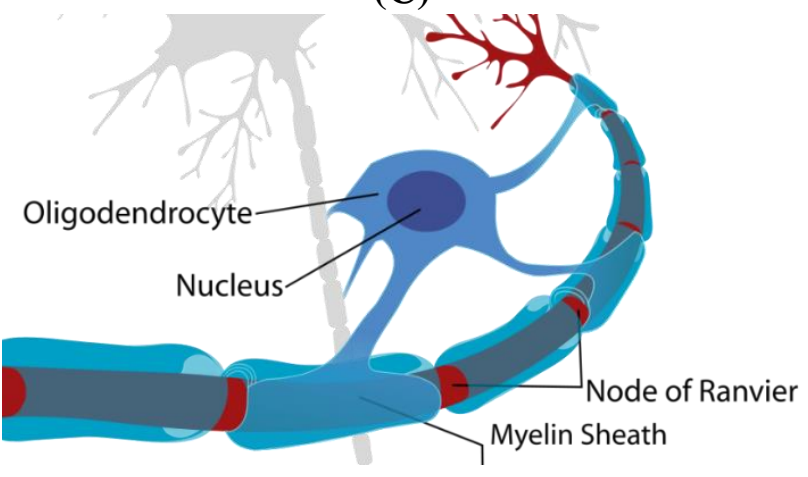

Figure 1-3: Illustrations of a neuron (A, Creative Commons), and two glial cell types (Wikimedia Commons): Astrocyte (B) and Oligodendrocyte. (C). 
White matter brain tissue consists of neurons that have myelinated axons which act as insulation for signal transmission (Sekhar et al., 2012). This tissue can be found in varying proportions with unmyelinated axons, or gray matter tissue, throughout the brain. The corpus callosum has the highest concentration of white matter neurons, while regions in the cerebrum and brainstem include a mix of both tissue types. Regions that are predominantly grey matter include the cortex, thalamus, and basal ganglia.

\subsubsection{Brain Material Properties}

While the anatomy and functional processes of the brain are complex, so is the mechanical behavior of the tissue. Brain tissue has been shown to be viscoelastic; both spatially and temporally nonlinear (Darvish and Crandall, 2001; Takhounts et al., 2003a), anisotropic (Arbogast and Margulies, 1998; Prange and Margulies, 2002), age-dependent (Gefen et al., 2003; Thibault and Margulies, 1998), and nearly incompressible, i.e., negligible volume change when deformed (McElhaney et al., 1976). Although many studies have characterized the mechanical response, no set of material properties has been universally accepted for the human brain (Hrapko et al., 2008).

The uncertainty in brain material properties is evident by the large range of values reported in the literature, e.g., the magnitude of the complex shear modulus spans nearly three orders of magnitude (Meaney et al., 2014). This variation can be attributed to several factors including interregional differences in the composition of the tissue, experimental methods used for testing, and the constitutive models used to characterize the mechanical response. For example, white matter regions are highly organized with preferred fiber directions, e.g., corpus callosum, making their response stiffer and anisotropic when compared to most gray matter regions which tend to be softer and isotropic (Hrapko et al., 
2008). Differences in experimental test methods have also affected the measurement of material properties, these include the extent, rate, and mode of mechanical loading (shear, compression, indentation, electrography), pre-compression, pre-conditioning, ambient temperature, and perfusion of the tissue (Hrapko et al., 2008). Furthermore, nerve tissue autolyzes within hours of death, which limits the time brain material properties can be reliably measured (Fountoulakis et al., 2001).

In regards to constitutive models, some have used linear viscoelastic formulations to characterize the mechanical behavior of brain under small deformations, while other have used quasilinear to fully nonlinear models for larger deformations. For example, Takhounts et al., 2003, proposed 20\% Lagrangian shear strain as an upper limit for applicability of a linear viscoelastic model, up to $50 \%$ strain for a quasilinear model, and for strains above $50 \%$, a fully nonlinear model was needed. However, despite the complexity of the brain and the variation of material properties, many have been able to validate FE brain models against limited datasets using linear viscoelastic and isotropic constitutive models, e.g., (Kimpara et al., 2006; Mao et al., 2013; Takhounts et al., 2008).

\subsubsection{Brain Injuries and Mechanisms}

This subsection provides background on various brain injury types, and their hypothesized mechanisms. Focus was placed on diffuse brain injuries (concussion and DAI). Only injuries due to impact or inertial loading of the head are discussed; this review does not include blast brain injuries or injuries involving skull fracture. Several studies are recommended for a more comprehensive review of brain injury mechanisms: (Meaney et al., 2014; Ommaya, 1984; Pudenz and Shelden, 1946). 
Brain injury is a type of traumatic head injury that consists of two primary classifications: closed and penetrating (Santiago et al., 2012). While penetrating brain injuries are accompanied by skull fracture, closed-head injuries involve little to no deformation of the skull. Closed-head injuries can be categorized into either focal or diffuse types: Focal injuries consist of regions that have highly localized patterns of brain damage. These injuries include contusions, lacerations, and hemorrhage (hematoma), and typically result from blunt head trauma (Santiago et al., 2012). Diffuse injuries are characterized by patterns of axonal damage spread across regions or throughout the entire brain (Gennarelli et al., 1982). These injuries include concussion and DAI, and are believed to result from rapid rotational motion of the head (Gennarelli et al., 1982). While focal injuries are easily identified under most imaging modalities, due to gross disruption of the tissue, diffuse injuries are more challenging to detect because the damage is primarily microscopic (Smith et al., 2003). Relative to focal injuries, which are typically severe, diffuse brain injuries span a continuum of severities from mild-to-severe (Figure 1-4).

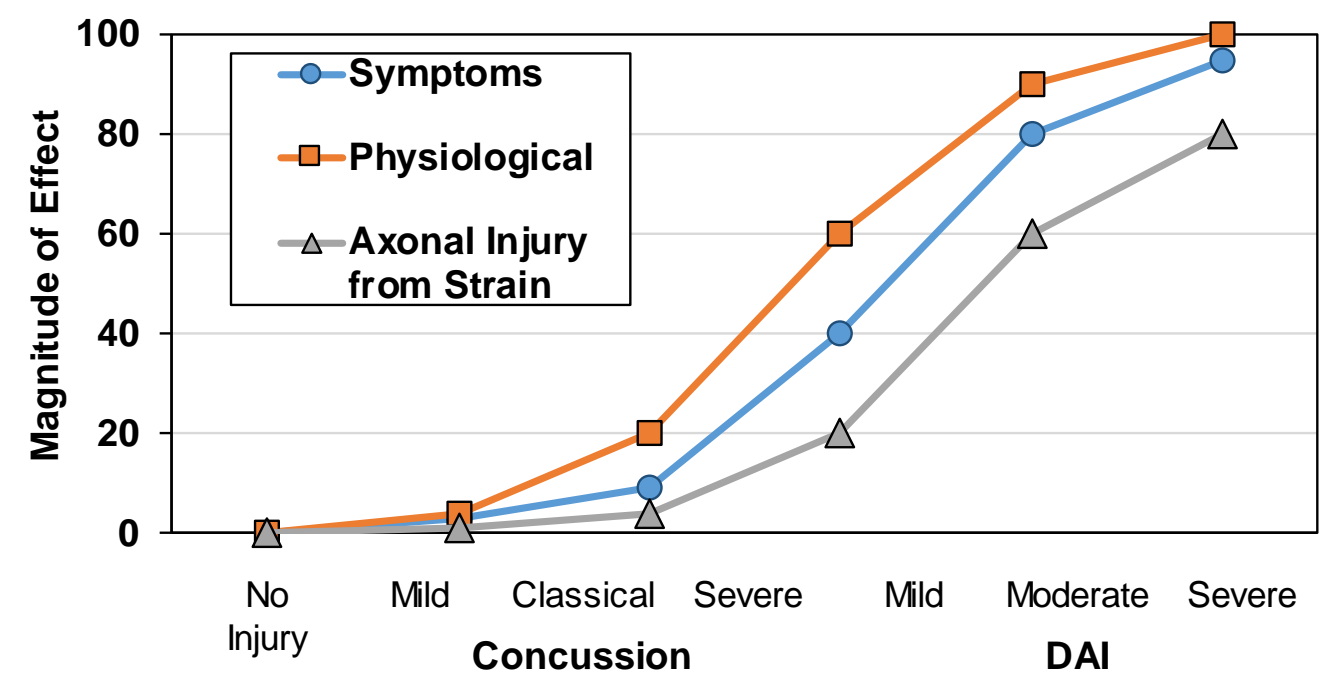

Figure 1-4: Continuum of diffuse brain injury (originally from Gennarelli 2015; IRCOBI Angular Head Motions Workshop). Lines plotted show the relative increase in magnitude of effect due to increase in injury severity. 
The earliest known brain injuries were recorded by the philosophers of ancient Greece. Most notably was the work of Hippocrates who wrote on his observations of concussion (i.e., to shake violently in Latin), stating that “...In cerebral concussion, whatever the cause, the patient becomes speechless...falls down immediately...cannot see or hear..." (McCrory and Berkovic, 2001). Although the definition of concussion has changed since then, the general symptoms that were described by Hippocrates, e.g., altered consciousness, remains a characteristic that defines the injury today (McCrory et al., 2017). Other early definitions of concussion were based primarily on observations of the patient's behavior following a blow. In the $16^{\text {th }}$ century, the term concussion or "commotion cerebri", was used to describe the symptoms of any brain injury in the absence of skull fracture, i.e., altered or loss of consciousness (Pare 1579). Then in the $19^{\text {th }}$ century the term was modified to distinguish it from more severe forms of traumatic cerebral injury involving bleeding (hemorrhages), which were believed to be related to prolonged symptoms and death (Hewitt 1861).

In the mid- $20^{\text {th }}$ century the terms acceleration, cerebral, compression, and focal concussion were proposed each with distinct mechanical mechanisms, clinical symptoms, and tissue-level injury patterns (Denny-Brown and Russell, 1941). These descriptions were intended to distinguish from "pure" or traditional concussion which was considered to be a mild injury only consisting of altered or loss of consciousness lasting less than one hour. While focal and compression concussions were used to describe lesions and hematomas (asymmetric), acceleration and cerebral concussions were terms used primarily to describe diffuse (symmetric) brain injuries (Ommaya and Gennarelli, 1974). In 1940, Goggio proposed a pressure gradient theory for coup-contrecoup brain injury (Goggio, 1941). This 
theory modeled the brain as column of fluid, which when impacted resulted in maximum hydrostatic pressure at one end causing the coup injury, and minimum pressure at the opposite, causing the contrecoup injury. Although this theory sparked a number of experimental and computation investigations, no evidence has been found to suggest that pressure results in injury to the brain (Nusholtz et al., 1984). One explanation for this finding is that the pressures required to alter functional capacity of neural tissue, without causing permanent damage ( 100MPa), far exceeds the pressures that have been measured in the human brain during impact (less than 1MPa) (Nahum et al., 1977; Trosseille et al., 1992; Zhao and Ji, 2016a).

In 1943, Holbourn proposed one of the most comprehensive theories for a brain injury mechanism. He stated that brain injuries were governed by Newton's laws of motion and the physical properties of the brain and skull (Holbourn, 1943). Using fundamental mechanics principles and a physical model of the head made of gelatin and wax, Holbourn argued that concussive brain injuries were caused by tissue-level deformation (shear strains) generated primarily by rotational head motion. Since the brain consists of mostly water, it behaves as a nearly incompressible material; the bulk modulus $\left(\sim 10^{9} \mathrm{~Pa}\right)$ is on the order of one million times larger than the shear modulus $\left(\sim 10^{3} \mathrm{~Pa}\right)$. As a result, shear strain would be the dominate mode of brain deformation, and shear strain is caused primarily by rotational head motion. Among his conclusions, Holbourn stated that "For blows of long duration, the shear-strains in the brain are proportional to the force..." (i.e., acceleration), while "...for very short duration blows the injury is proportional to the force multiplied by the time for which it acts." (i.e., change in velocity). This statement is a subject of investigation in the following chapters of this dissertation. 
Numerous studies attempted to confirm Holbourn's hypothesis, following his seminal paper in 1943, e.g., (Gennarelli et al., 1971; Ommaya and Gennarelli, 1974; Ommaya et al., 1964; Pudenz and Shelden, 1946). Most notably was work by Ommaya et al., 1964 and Gennarelli et al., 1971, which provided experimental evidence that supported Holbourn's theory, and suggested rotational head motion was the primary mechanism for cerebral concussion. Later, in 1974, Ommaya and Gennarelli proposed what would become known as the centripetal theory of concussion (CTC), which was defined as "... a graded set of clinical syndromes...caused by mechanically induced strains affecting the brain in a centripetal sequence of disruptive effect on function and structure." They then hypothesized shear strain caused by rotational head motion to be the mechanism of cerebral concussion, where strains are initiated at the periphery (cortex) and propagate inward to the center of the brain (Ommaya and Gennarelli, 1974).

Today, the definition of concussion has broadened considerably. Prior to the 1970s, concussion was defined symptomatically as a loss of conscious (Ommaya and Gennarelli, 1974); however, modern definitions no longer require loss of consciousness, and include any state of altered consciousness following impact; drowsiness, headache, irritability, etc. (McCrory et al., 2017). Unfortunately, the lack of a concise definition for concussion further complicates an already challenging problem for clinicians, researchers, and engineers. Sport-related concussion is defined as "a traumatic brain injury induced by biomechanical forces" that may be caused by either a direct or indirect blow to the head, and often "results in the rapid onset of short-lived impairment of neurological function that resolves spontaneously" (McCrory et al., 2017). Concussions are typically identified using a battery of tests that assess symptoms, neurocognitive capacity, and motor function; some 
examples include the Glasgow Coma Scale (Teasdale and Jennett, 1974), the Standardized Assessment of Concussion (McCrea et al., 1998), and the Immediate Postconcussion and Cognitive Testing battery (ImPACT). These tests have shown to be practical and effective for screening of a suspected concussion; however, their efficacy may be improved with an appropriate biomechanical assessment. This could be accomplished using wearable sensors, which would allow for real-time screening of concussion. Existing tolerance criteria for wearable sensors are based on head kinematic measurements that have been linked to clinical outcomes identified via neurocognitive assessments and imaging (Camarillo et al., 2013; Elliott et al., 2015). Thus, this approach could be used to better identify concussed players for subsequent clinical evaluation and treatment.

\subsubsection{Brain Injury Criteria}

Injury criteria are used to evaluate the efficacy of safety countermeasures and protective equipment. Injury criteria relate biomechanical parameters of impact typically measured using an anthropometric test device (ATD or dummy) to a probability of injury. For example, US government regulatory crash tests use a standardized $\left(50^{\text {th }}\right.$ percentile $)$ human male test dummy (Hybrid-III) for evaluating the crashworthiness of automobiles (NHTSA). Injury criteria are typically developed based on human injury tolerances derived from experimental data. However, limitations with testing human tissues may make it challenging to obtain adequate data for proper development. Under these circumstances, alternative approaches involving scaling of animal data and/or computational modeling are used. Injury criteria for various body regions have been proposed, e.g., head, brain, neck, thorax, and leg, and several are currently used in regulatory and consumer testing. 
Brain injury criteria are responsible for assessing brain injury risk during head impact. They are used in testing with dummies to evaluate the safety of countermeasures and head protective equipment e.g., (National Operating Committee on the Standards for Athletic Equipment (NOCSAE), 2012; NHTSA, 2015; Rowson and Duma, 2011); however, they have also been used to investigate brain injury mechanisms in living humans using wearable sensors (Allison et al., 2014, 2015; Bartsch et al., 2014; Duma et al., 2005; Wu et al., 2016). Brain injury criteria consist of a biomechanical metric and an injury risk function. The metric summarizes the severity of head impact, and is a mathematical function consisting of one or more biomechanical response variables. Metrics used for brain injury criteria can be categorized into two types: kinematic-based and tissue-levelbased. Kinematic metrics are based on rigid body parameters of the head, while tissue-level metrics are based on mechanics of the parenchyma. The risk function is a probabilistic model that relates the metric to brain injury likelihood. While the risk curve is necessary for predicting brain injury, the underlying metric is responsible for representing the injury mechanism and relative severity of head impact.

\subsubsection{Translational Kinematic Metrics}

Head kinematics have been the basis for most head and brain injury metrics. This is due to the feasibility of measuring and summarizing head kinematic response, either on a dummy or a volunteer, relative to measuring brain tissue response. Head kinematics can be separated into two different types of motion: translational and rotational. Early metrics were formulated using only translational parameters of head motion, and brain injuries were inferred using head injury criteria, which were developed for assessment of skull fracture and facial injuries. For example, NOCSAE utilizes a metric that is based on the 
time history of resultant linear head acceleration, the severity index (Gadd, 1966), for helmet certification, while the National Highway Traffic Safety Administration (NHTSA) uses a modified version of the severity index for regulating automobile crashworthiness, the Head Injury Criterion (HIC) (Versace, 1971), for (Department of Transportation NHTSA Docket Number 69-7, Notice 19). While translational kinematic parameters may be good indicators of head impact severity and skull fracture risk, they are not suitable for assessing all types of brain injury (Margulies and Thibault, 1992; Takhounts et al., 2013).

\subsubsection{Rotational Kinematic Metrics}

Given Holbourn's hypothesis and the physical properties for the brain-skull system, rotational head kinematics seem well suited for studying brain injuries due to closed-head impact. Since 1943, numerous studies have focused on developing rotational brain injury tolerances using experimental data and/or computational models, e.g., (Kimpara and Iwamoto, 2012; Ommaya and Hirsch, 1971; Rowson et al., 2012; Takhounts et al., 2013). Some of the first advanced brain injury criteria that included rotational kinematic parameters in the mathematical form of the metric were accompanied by translation parameters. These metrics were often based on a combination of resultant linear and resultant angular head acceleration (Newman, 1986; Newman et al., 2000; Rowson and Duma, 2013), while some included empirically derived combinations of rotational parameters and HIC (Greenwald et al., 2008; Kleiven, 2007). As experimental and computational evidence mounted supporting rotational head motion as a brain injury mechanism, translational kinematic parameters were excluded from the mathematical form of the metric, and brain injury criteria were based solely on rotational kinematics (Kimpara and Iwamoto, 2012; Takhounts et al., 2013; Yanaoka et al., 2015). 


\subsubsection{Finite Element Brain Models}

While direct measurement of tissue-level brain mechanics remains challenging, the use of validated, anatomically detailed finite element (FE) brain models provides a useful alternative. A number of FE brain models have been developed from various research groups; some examples are cited (Kleiven, 2007; Mao et al., 2013; Takhounts et al., 2008). Although many were validated against the same experimental dataset, evidence suggests that the tissue-level responses between different FE models can vary substantially (Ji et al., 2014). Factors that may explain this variation include differences in material properties, differences in geometry and mesh size/quality, in addition to the limited amount of data that is currently available for FE model validation.

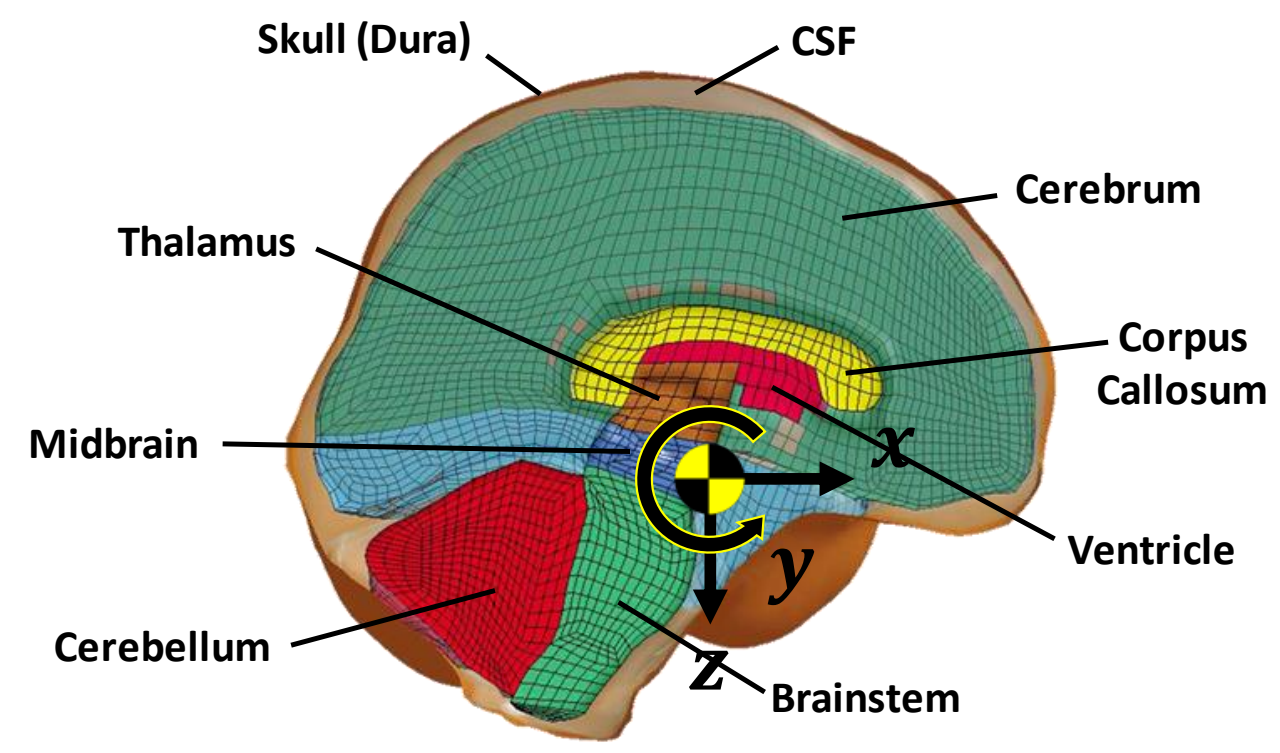

Figure 1-5: Mid sagittal plane view of the Global Human Body Models Consortium (GHBMC) FE brain model, and its local head coordinate system. Bridging veins, ventricles and meninges are not shown.

A typical FE model head impact simulation involves applying six degree-offreedom (6DOF) head kinematics to an anatomically derived coordinate system that is coupled to a rigid skull (Figure 1-5). The resulting rigid body motion of the head causes 
the brain elements to deform, allowing for measurement of tissue-level mechanical responses. The advantage of using an FE model over a kinematic-based metric for brain injury assessment is the FE models ability to measure the complex spatial and temporal mechanical response of the brain during the impact, whereas a kinematic-based metric uses peaks from the time history to estimate brain responses. To summarize FE model output for injury assessment, several metrics based on tissue-level responses have been proposed, e.g., maximum hydrostatic pressure, stress (von Mises), strain, strain-rate, and their product. Although these metrics are simulation-based, they have been used extensively in TBI-related biomechanics research, and thus FE brain models are considered to be stateof-the-art injury risk assessment tools (Deck and Willinger, 2009).

One current limitation of FE models is their long computation time relative to kinematic-based metrics. FE simulations require several hours to simulate a single head impact $(\sim 100 \mathrm{~ms})$, which make them impractical for rapid risk assessment in regulatory testing or in on-field evaluations using wearable sensors. Ideally, a brain injury criterion should be able to predict tissue-level response with the accuracy of an FE model, while retaining the computation time of a kinematic-based metric.

\subsubsection{Brain Injury Criterion (BrIC)}

Recently, several studies have leveraged FE brain models to develop kinematicbased metric-formulations for brain injury criteria (Kleiven, 2007; Takhounts et al., 2013; Yanaoka et al., 2015). Most notably was the development of the Brain Injury Criterion (BrIC) by Takhounts et al., 2013. This seminal study was the culmination of more than a decade of research and development on brain injury criteria, resulting in multiple publications (Takhounts et al., 2003b, 2008, 2011). Motivation for the development of 
BrIC emerged out of a need for supplementing existing head injury risk assessments based solely on translational head motion (HIC) with a rotational criterion for use in crashworthiness evaluation (NHTSA, 2015). Despite safety requirements used in the design of automobiles, i.e., HIC, brain injuries remain one of the most frequently injured and cost incurring body regions in car crashes (Eigen and Martin, 2005). While Takhounts proposed several reasons for this phenomenon, he believed that the interpretation of the dummy's measurements was insufficient to capture all types of TBI occurring in the field (Takhounts et al., 2013). The development of BrIC was a process involving two steps:

The first step involved making use of available experimental brain injury data from animal studies. Using established scaling techniques, animal head kinematics measured during impact were scaled to their human equivalent. The data was then simulated in FE brain models to obtain tissue-level responses; Maximum Principal Strain (MPS) and Cumulative Strain Damage Measure (CSDM). These two metrics, which were previously established as indicators of DAI and severe concussion (Bandak, 1995; Takhounts et al., 2008), were used as predictor variables in two separate injury risk functions.

The second step involved correlation of MPS and CSDM with head kinematic parameters obtained from a dataset of more than 400 occupant crash and impactor tests performed with various dummies. Correlations with both rotational and translational parameters of head motion (velocity and acceleration) were investigated. The metric that established the highest correlation between kinematics, and MPS and CSDM was BrIC; a mathematical function of the directionally dependent magnitudes of the three axis head angular velocities, which were each weighted by critical value that was based on a FE brain 
model. The link between BrIC and brain injury risk was then established through linear regressions with strain-based brain injury criteria (MPS and CSDM).

\subsubsection{Multibody models}

Multibody (MB) models have been used extensively in biomechanics research to study human tolerance to impact, e.g., (Brinkley and Shaffer, 1971; Kim et al., 2013; Lobdell et al., 1973). These models consist of mass $\left(m_{i}\right)$, stiffness $\left(k_{i}\right)$, and damping $\left(c_{i}\right)$ elements, which are excited by motion variables $\left(\ddot{x}_{i}, \dot{x}_{i}, x_{i}\right)$, and have been used to obtain mechanical response correlates for injury prediction (Figure 1-6, $i=1,2$ ). Although early head and brain injury tolerances were based on correlations with MB response (Brinn and Staffeld, 1970; Fan, 1971; Slattenschek and Tauffkirchen, 1970; Stalnaker et al., 1971), they have not been leveraged in the development of more recent brain injury criteria. It is possible that this overlook was due to the adoption of HIC in 1971 by NHTSA, who, given their leadership in biomechanics research at the time may have steered the field toward the development and use of empirically derived brain injury metrics (Department of Transportation NHTSA Docket Number 69-7, Notice 19).

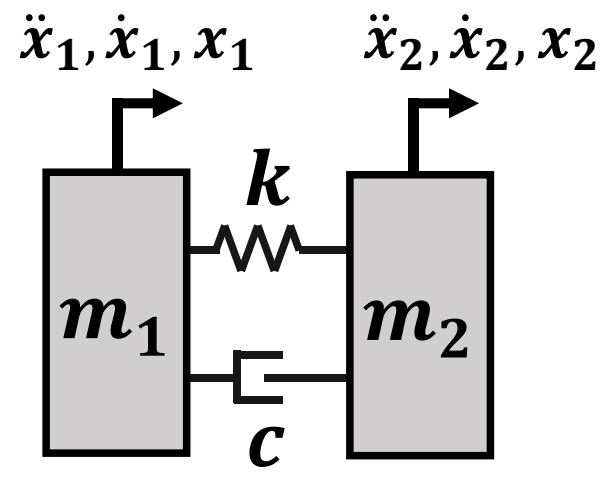

Figure 1-6: MB model used for the Mean Strain Criterion (Stalnaker et al., 1971).

In regards to brain injury criterion development, $\mathrm{MB}$ models may be the most effective tools for rapidly estimating brain deformations during impact. Although tissue- 
level metrics are preferred for brain injury assessment, they require FE model simulation, and are thus the most time-consuming to obtain. On the other hand, kinematic-based metrics are less-time consuming but also less accurate than tissue-level metrics. MB models provide an intermediate approach, and can be used to rapidly estimate FE model response over a broad range of head impacts (Figure 1-7).

\section{$R^{2}=1$}

\section{Correlation with Brain Strain}

\section{$R^{2}<1$}

FE Models

MB Models

Kinematic Metrics

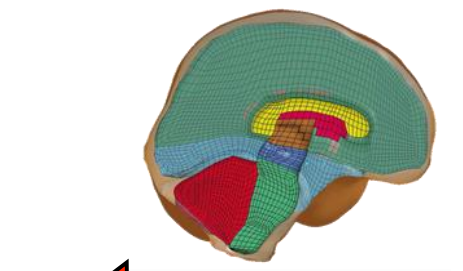

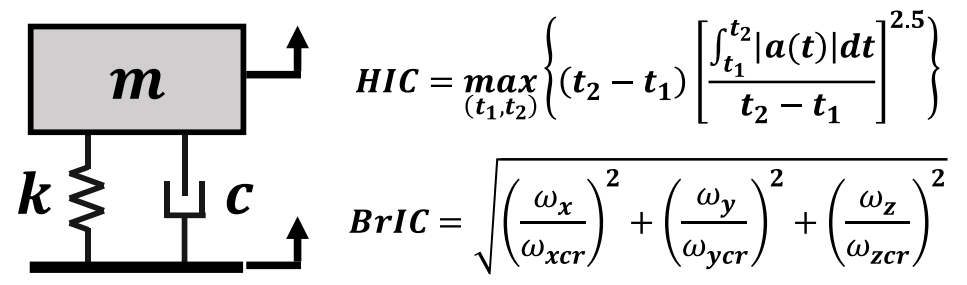

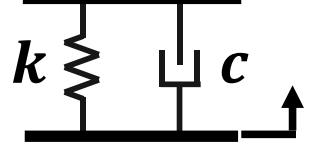

\section{Hours}

Computation Time Instantaneous

Figure 1-7: Tools that can be used to model brain deformation mechanics, and their relative location on the spectrum of accuracy versus computational cost.

\subsubsection{Summary}

The following points are intended to summarize the key observations from the background research review:

- $\quad$ TBI is a leading cause of injury, and is increasing globally and in the US.

- Closed-head impacts account for most TBIs, and consist of diffuse and focal types.

- Diffuse brain injuries include concussion and DAI, are currently the most common brain injury types sustained in automotive-and sport-related impacts.

- Head and brain injury criteria assess the efficacy of safety countermeasures and head protective equipment, and include a biomechanical metric and an injury risk function. 
- Metrics can be categorized into two types; kinematic-based and tissue-level-based.

- Existing kinematic-based metrics used in crashworthiness assessment and helmet certification are based on translational kinematics only.

- Brain deformation is the primary mechanism for closed-head impact brain injuries.

- Direct measurement of brain strain is not possible with existing ATDs and requires FE model simulation of head impact, which incur substantial computational cost.

- Rotational, not translational head motion is the mechanism for brain strain.

- Correlations between rotational kinematic parameters and FE brain strains have been used to develop brain injury criteria targeting tissue level injuries.

- Existing strain-based metrics depend on the direction of head impact and show high correlation with angular velocity, and low correlation with linear acceleration.

- MB models have been used to study human injury tolerances, including skull fracture, and may be useful for developing effective brain injury criteria.

\subsection{Motivation}

\subsubsection{Limitations with Existing Brain Injury Criteria}

Although BrIC was shown to be a good predictor of strain-based metrics in the occupant crash test environment, it's efficacy over a broader range of loading conditions raises concern. Currently, the mathematical form of BrIC includes head angular velocity only. While several experimental and computation studies suggest that angular velocity is an important parameter for predicting brain injury, many of these studies also suggest that, in addition to angular velocity, brain injury tolerance depends on angular acceleration and/or the duration of head impact e.g., (Kimpara and Iwamoto, 2012; Margulies and Thibault, 1992; Ommaya et al., 1967; Yanaoka et al., 2015). Injury tolerances in other body 
regions, including the skull, lower limb, spine, pelvis, muscle, ligament, etc. have also demonstrated a dependence on both the magnitude and duration of loading, and in some cases injury criteria have been developed to reflect these mechanical dependencies. Thus, both the magnitude and duration of a rotational head impact pulse should be investigated for potential inclusion in the mathematical form of a brain injury criterion metric.

Angular acceleration and/or impact duration have been considered in the development of several previous brain injury criteria. For example, a preliminary version of BrIC involved a linear combination of the maximum resultant angular velocity and angular acceleration (Takhounts et al., 2011), while several other metrics maximize an integration over the time history of angular acceleration (similar to HIC) to establish brain injury predictors (Kimpara and Iwamoto, 2012; Yanaoka et al., 2015). Although each of these metrics have demonstrated efficacy for predicting brain injury responses within the dataset used for their development, their performance over a broader range of head impact conditions remains questionable. Furthermore, with an increased focus on developing advanced helmet technologies, integrated safety systems, and autonomous vehicles, limitations with existing brain injury criteria will be tested by pushing their use into loading scenarios where they may be less accurate. In these situations, existing criteria my not affect design as intended, or in a potentially worst-case scenario, promote improper design.

\subsubsection{Need for a Universally Applicable Brain Injury Criterion}

Given the various kinematic-based head and brain injury criteria that have been proposed, it is not always clear which metric should be used to assess the severity of a head impact for a particular loading condition. The metric that establishes brain injury risk is some mathematical combination of the velocity and/or acceleration components of 
translational and/or rotational head motion. While Holbourn's theory supports the use of rotational head kinematics for brain injury assessment, the relationship between angular velocity, angular acceleration, and/or duration in the mathematical form of the metric is not clear. Furthermore, most existing kinematic brain injury criteria utilize empiricallyderived metrics based on subsets of head impact data. Although empirical approaches are favored for their ability to quickly describe trends in the data, they are often developed from the "best fit" of one or a combination of input variables, which may be highly correlated within a limited dataset. However, these correlations may not hold outside the experimental dataset, and an empirically-derived injury criterion can lead to nonsensical predictions when extrapolated beyond the range of data used to fit the model. Ideally, a universally applicable brain injury criterion should be derived from fundamental mechanical principles first, and then verified with a broad range of experimental data, rather than being developed from subsets of data using statistical models.

\subsection{Scope}

\subsubsection{Goal and Aims}

The goal of this dissertation is to develop improved metrics for brain injury criteria that are universally applicable in a broad range of closed-head impact conditions. The proposed metrics will be formulated using fundamental mechanics principles, and capable of predicting strain-based brain injury responses (MPS and CSDM) from FE model simulation of a broad range of head impacts. The aims of this dissertation are:

1. Assemble a large database of head impact kinematics and brain strain responses from diverse impact conditions. 
2. Assess existing kinematic-based metrics to identify key head kinematic parameters for predicting brain strain response in various impact modes.

3. Develop a theoretical framework to establish a relationship between key head kinematic parameters and brain deformation.

4. Develop and assess kinematic-based and MB model-based metrics for rapid prediction of FE brain strain response to head impact.

\subsubsection{Disclaimer}

This dissertation focuses on the development of the biomechanical metric only (Figure 1-8; Kinematics to Strain, Section 1.2.8); no new brain injury risk functions have been developed. Currently there is a lack of well characterized human brain injury datasets, i.e., existing datasets are garnered from either scaled animal data, or from reconstructions and/or wearable sensors involving humans, which make it difficult to develop injury criteria with reasonable confidence (Sanchez et al., 2017). While methods to acquire more reliable data from these sources are improving, the process may take considerable time. For this dissertation strain-based brain injury metrics (MPS and CSDM) predicted by the proposed brain injury criterion are assumed to be representative of (well correlated with) diffuse brain injury types that occur in closed-head, inertial impact scenarios. These injuries include mild-to-severe concussion and DAI (Figure 1-4), which are believed to be caused by excessive brain deformation (Gennarelli et al., 1998), and have been shown to be the most common brain injury types sustained in automotive (Gennarelli, 1993; Kameyama et al., 2008) and sport-related (Harmon et al., 2013) head impact environments. Thus, the proposed metrics are intended to be universally applicable for assessing diffuse brain injury types from head impacts occurring in these loading environments. 


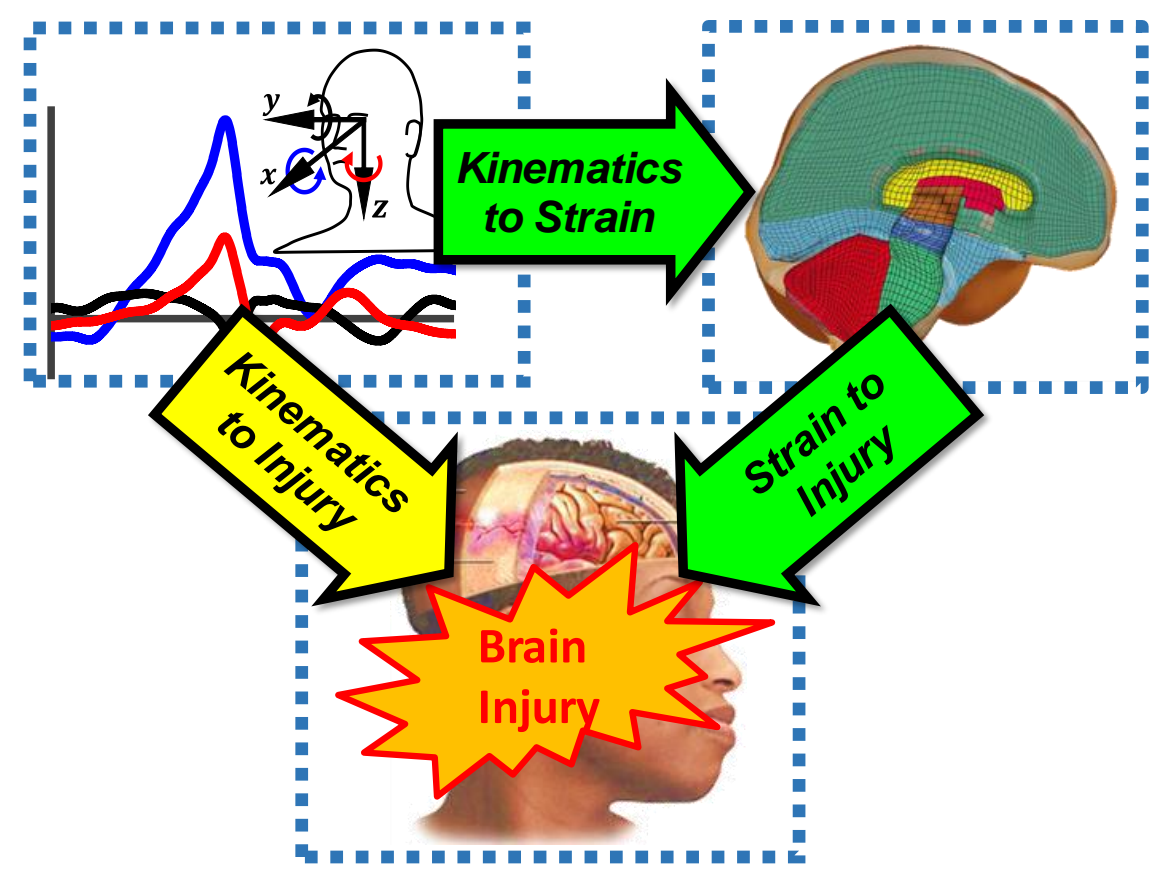

Figure 1-8: Approach for developing kinematic-based brain injury criteria based on tissue-level responses, e.g., Takhounts et al., 2013. The link between head kinematics and brain injury (yellow arrow) is established through correlations between head kinematic parameters and FE brain strains (Kinematics to Strain), and FE brain strains and brain injuries (Strain to Injury) (green arrows). This dissertation focuses on improving the Kinematics to Strain relationship. Bottom image was obtained from (LifeART).

\subsubsection{Approach and Chapters}

The metrics proposed in this dissertation will be developed through an approach involving several steps, each presented in a separate chapter. An overview of the data and methods used throughout this dissertation is provided in Chapter 2 (aim 1). Existing kinematic-based metrics are assessed in Chapter 3 (aim 2). The theoretical framework used for developing the brain injury criterion metrics is presented in Chapter 4 (aim 3). The development and validation of the proposed kinematic and MB model-based metrics are presented in Chapters 5 and 6, respectively (aim 4). Additional data, methods, results, and analysis can be found in the appendices. 


\section{Chapter 2 - Data and Methods}

This chapter provides details on the data collected and methods used for this dissertation. A description of the impact conditions and head kinematics data is provided in Section 2.1. Details on the finite element human brain models, head impact simulations, and strain-based metrics are described in Section 2.2.

\subsection{Head Kinematics}

\subsubsection{Head Impact Database}

A comprehensive database of timed, 6DOF head impact kinematics was assembled for use in this dissertation. The database consists head impacts from automotive sled and crash tests involving dummies and cadavers used as occupants and pedestrians, impactor tests performed at multiple locations on both helmeted (American football) and unhelmeted dummies, and head motions from human volunteer response to sled acceleration (Figure 2-1). A brief description of each impact condition is provided below and summarized in Table 2-1. A list of the specific crash and volunteer tests used is provided in Appendix I. Data was obtained either from published studies or publically available databases. In total, 1747 head impacts have been collected and used for this dissertation.

Frontal sled tests: Head kinematics from front driver and rear passenger dummy sled tests were collected from studies investigating occupant response in frontal impact. The front driver tests were part of the first and second universal gold standard series used to evaluate dummy biofidelity in frontal impact (Crandall, 2013), while occupant sensitivity to various restraints was the focus of the rear passenger tests (Forman Jl et al., 2013). Head motion in these events was primarily inertia based, since neither airbag nor compartment interactions occurred. 
Frontal crash tests: Dummy head kinematics were collected from NHTSA's frontal NCAP, and the Insurance Institute for Highway Safety's (IIHS) small and moderate overlap impact crash test programs. The NCAP tests involved collision of a passenger vehicle, head on, into a rigid barrier at $35 \mathrm{mph}$, while the IIHS tests consisted of a vehicleinto rigid barrier at $40 \mathrm{mph}$ with either a $25 \%$ (small) or $40 \%$ (moderate) overlap. Head kinematics were measured using the Hybrid-III $50^{\text {th }}$ male test dummy.

Oblique sled tests: Sled tests performed on cadavers and dummies in $20^{\circ}$ and $60^{\circ}$ far side oblique configurations were collected. Surrogates used in the $20^{\circ}$ condition were tested in a 2014 Hyundai Elantra Buck (Crandall, 2014), while a custom sled-buck was used for the $60^{\circ}$ condition (Forman Jl et al., 2013). Test subjects were exposed to simulated vehicle closing speeds between (9-35) mph. In the $20^{\circ}$ condition, occupants sustained impacts to the head through airbag and compartment interactions, while no head impacts occurred in the $60^{\circ}$ condition.

Oblique crash tests: A number of frontal offset (oblique) crash tests from NHTSA's research and development $(\mathrm{RD})$ program were collected. These include both vehicle-tovehicle and oblique moving deformable barrier (OMDB) crashes (Saunders et al., 2015). Tests consisted of varying front end offset angles and overlaps with closing speeds between (55-70) mph. The OMDB test configuration has been proposed in a recent update to the New Car Assessment Program (NCAP) and will include BrIC for brain injury risk assessment (NHTSA, 2015). Head kinematics were measured using the Test device for Human Occupant Restrain (THOR) $50^{\text {th }}$ male test dummy.

Side sled tests: Fourteen sled tests were performed on three cadavers in a pure lateral $\left(90^{\circ}\right)$ far side condition (Forman $\mathrm{Jl}$ et al., 2013). The sled buck and test conditions 
used were similar to those from the $60^{\circ}$ far side oblique configuration with simulated closing speeds between (10-20) mph. No direct head impacts occurred in these tests.

Side crash tests: Crash tests from NHTSA's NCAP, and IIHS's side impact programs were collected. The NCAP condition involved both moving deformable barrier and rigid pole tests, while the IIHS crash tests were all moving barrier tests. Impacts were performed at $0^{\circ}$ and $25^{\circ}$ orientations relative to a vector normal to the side of the vehicle. Head kinematics were measured using the World-SID and Euro-SID $50^{\text {th }}$ male and $5^{\text {th }}$ female test dummies.

Pedestrian sled tests: Head kinematics from 28 sled tests were collected from three separate studies investigating vehicle-to-pedestrian interaction in frontal impact (Kerrigan et al., 2012, 2008; Subit et al., 2008). Stationary cadavers and dummies were impacted laterally in a standing posture by a sled-buck that was modified with various passenger vehicle front ends (Sedan and SUV). Closing speeds were varied between (20-35) mph.

Un-helmeted impactor tests: The un-helmeted impactor (Pendulum) tests were obtained from Takhounts et al., 2013 where several different crash test dummy head forms were rigidly mounted to a fixed support at the base of the neck, and impacted at various angles and energies using a pneumatically driven impactor (Takhounts et al., 2013). These tests were organized into the separate impact conditions based on the impact angle relative to the dummy head, where $0^{\circ}$ impacts were considered frontal, $30^{\circ}$ and $60^{\circ}$ considered oblique, and $90^{\circ}$ considered side impacts.

Helmeted impactor tests: Of the more than 1800 helmeted impactor tests conducted by Viano et al., 2012, a subset of 576 impacts at 5.5, 7.4, and $9.3 \mathrm{~m} / \mathrm{s}$ at eight locations on twenty-four different helmets were used in this dissertation. Helmet impact locations and 
speeds were based on video analysis of professional football players; where $9.3 \mathrm{~m} / \mathrm{s}$ was the average on-field closing speed for concussion (Viano et al., 2012).

Human Volunteer tests: The human volunteer data were obtained from a study which performed an evaluation of brain injury criteria using 335 separate tests involving twenty adult male volunteers subjected to low-level sled acceleration (Sanchez et al., 2017). Volunteers were tested in three different configurations: front-facing rearward acceleration at 0 , oblique at 45 , and pure lateral (side) at $90^{\circ}$. The data were originally obtained from a much larger test series performed by the Naval Biodynamics Laboratory (NBDL) (Ewing and Thomas, 1972).

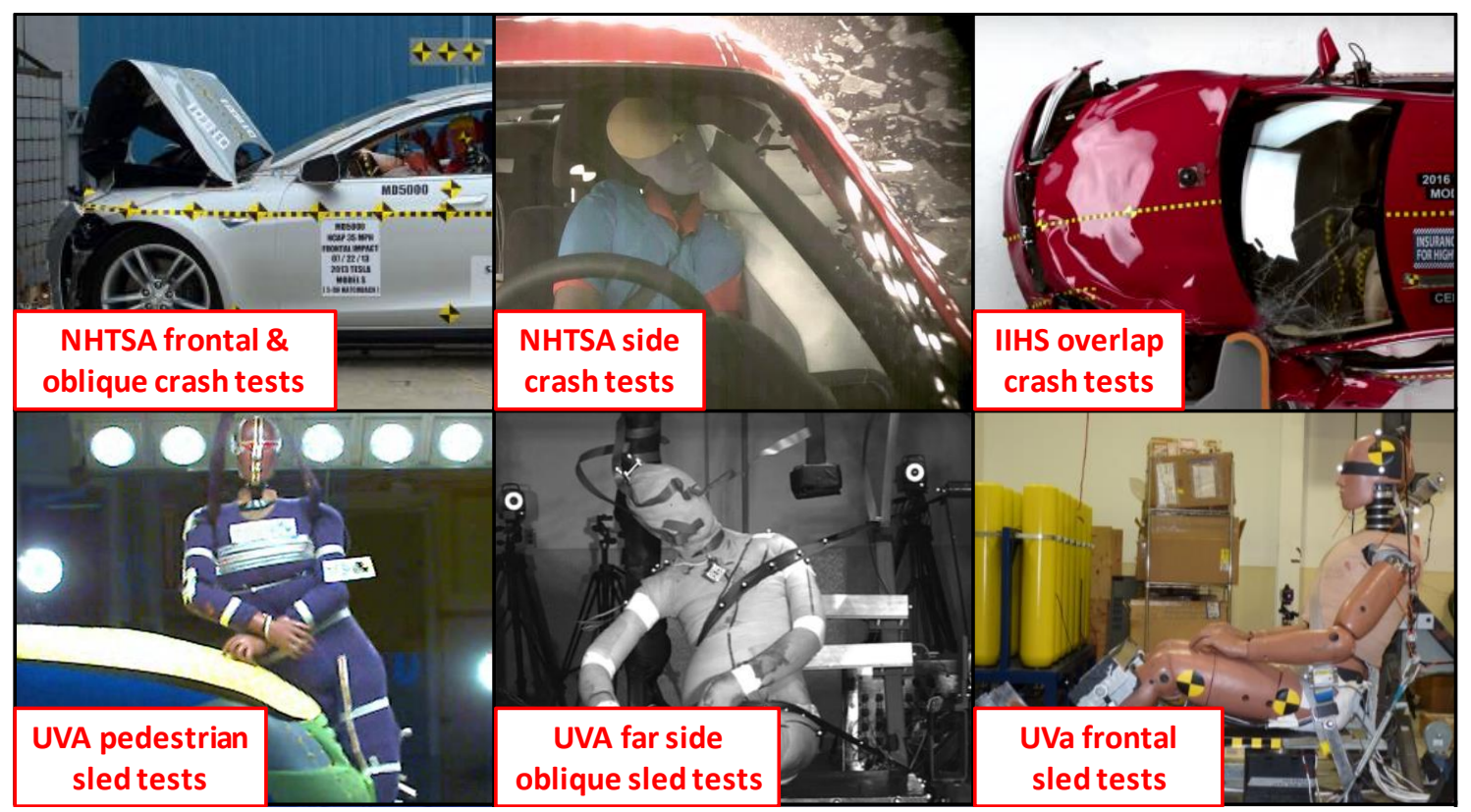

Figure 2-1: Examples of impact conditions included in this dissertation. 
Table 2-1: Summary of the impact conditions included in this dissertation.

\begin{tabular}{|c|c|c|}
\hline $\begin{array}{l}\text { Impact } \\
\text { category } \\
\text { (sample size) }\end{array}$ & $\begin{array}{l}\text { Impact condition } \\
\text { (sample size) }\end{array}$ & Test details (sample size) \\
\hline \multirow{4}{*}{$\begin{array}{l}\text { Sled tests } \\
\quad(445)\end{array}$} & $\begin{array}{l}\text { Frontal (FRT) } \\
\qquad(169)\end{array}$ & $\begin{array}{l}\text { UVa driver side universal gold standard (13) } \\
\text { UVa rear seat occupant full frontal (24) } \\
\text { NBDL volunteer } 0^{\circ} \text { rearward acceleration (132) }\end{array}$ \\
\hline & $\begin{array}{l}\text { Oblique (OBL) } \\
\quad(128)\end{array}$ & $\begin{array}{l}\left.\text { UVa } 20^{\circ} / 60^{\circ} \text { far side oblique ( } 31\right) \\
\text { NBDL volunteer } 45^{\circ} \text { oblique acceleration (97) }\end{array}$ \\
\hline & $\begin{array}{l}\text { Side (SID) } \\
\quad(120)\end{array}$ & $\begin{array}{l}\mathrm{UVa} 90^{\circ} \text { far side pure lateral }(14) \\
\text { NBDL volunteer } 90^{\circ} \text { pure lateral acceleration (106) }\end{array}$ \\
\hline & $\begin{array}{l}\text { Pedestrian (PED) } \\
\quad(28)\end{array}$ & UVa vehicle buck laterally into pedestrian (28) \\
\hline \multirow{3}{*}{$\begin{array}{l}\text { Crash tests } \\
\quad(533)\end{array}$} & $\begin{array}{l}\text { FRT } \\
(260)\end{array}$ & $\begin{array}{l}\text { NHTSA NCAP driver (46) } \\
\text { NHTSA NCAP front passenger (35) } \\
\text { NHTSA RD driver and rear passenger (28) } \\
\text { IIHS small \& moderate overlap impact driver (151) }\end{array}$ \\
\hline & $\begin{array}{l}\text { OBL } \\
(184)\end{array}$ & $\begin{array}{l}\text { NHTSA RD driver near and far side (102) } \\
\text { NHTSA RD front passenger near and far side }(57) \\
\text { NHTSA RD rear passenger near and far side }(25)\end{array}$ \\
\hline & $\begin{array}{l}\text { SID } \\
(89)\end{array}$ & $\begin{array}{l}\text { NHTSA NCAP driver near side (33) } \\
\text { NHTSA NCAP rear passenger near side (10) } \\
\text { NHTSA RD driver and rear passenger near side (25) } \\
\text { IIHS driver and rear passenger near side (21) }\end{array}$ \\
\hline $\begin{array}{l}\text { Impactor } \\
\quad \text { tests } \\
(769)\end{array}$ & $\begin{array}{l}\text { Frontal (74) } \\
\text { Oblique (78) } \\
\text { Side (41) } \\
\text { A (72) } \\
\text { B (72) } \\
\text { C (72) } \\
\text { D (72) } \\
\text { F (72) } \\
\text { R (72) } \\
\text { UT (72) } \\
\text { AP (72) }\end{array}$ & $\begin{array}{l}\text { NHTSA } 0^{\circ} \text { pendulum into head CG/Forehead } \\
\text { NHTSA } 30^{\circ} / 60^{\circ} \text { pendulum into head CG/Forehead } \\
\text { NHTSA } 90^{\circ} \text { pendulum into head CG } \\
\text { Biokinetics oblique facemask } \\
\text { Biokinetics upper oblique facemask } \\
\text { Biokinetics side of facemask } \\
\text { Biokinetics rear boss of shell } \\
\text { Biokinetics front of shell } \\
\text { Biokinetics rear of shell } \\
\text { Biokinetics side of shell } \\
\text { Biokinetics lower central facemask }\end{array}$ \\
\hline
\end{tabular}

NHTSA impactor tests were unhelmet; Biokinetics tests were helmeted; University of Virginia (UVa). Head kinematics for the crash tests are available to the public by download at (NHTSA.gov) and (techdata.iihs.org). Center of gravity (CG). 


\subsubsection{Head Kinematic Parameters}

Head kinematics were processed for calculation of kinematic-based metrics and FE model head impact simulations (Figure 2-2, A). Processed 6DOF time histories in the form of three linear accelerations and three angular velocities were determined from the raw sensor data in the local head anatomical coordinate system with an origin fixed at the head center-of-gravity (CG) and orientations defined by SAE-J211 (SAE International): $x$ pointing posterior to anterior, $y$ pointing left to right, $+z$ distal to proximal (Figure 2-2, B).

(A)

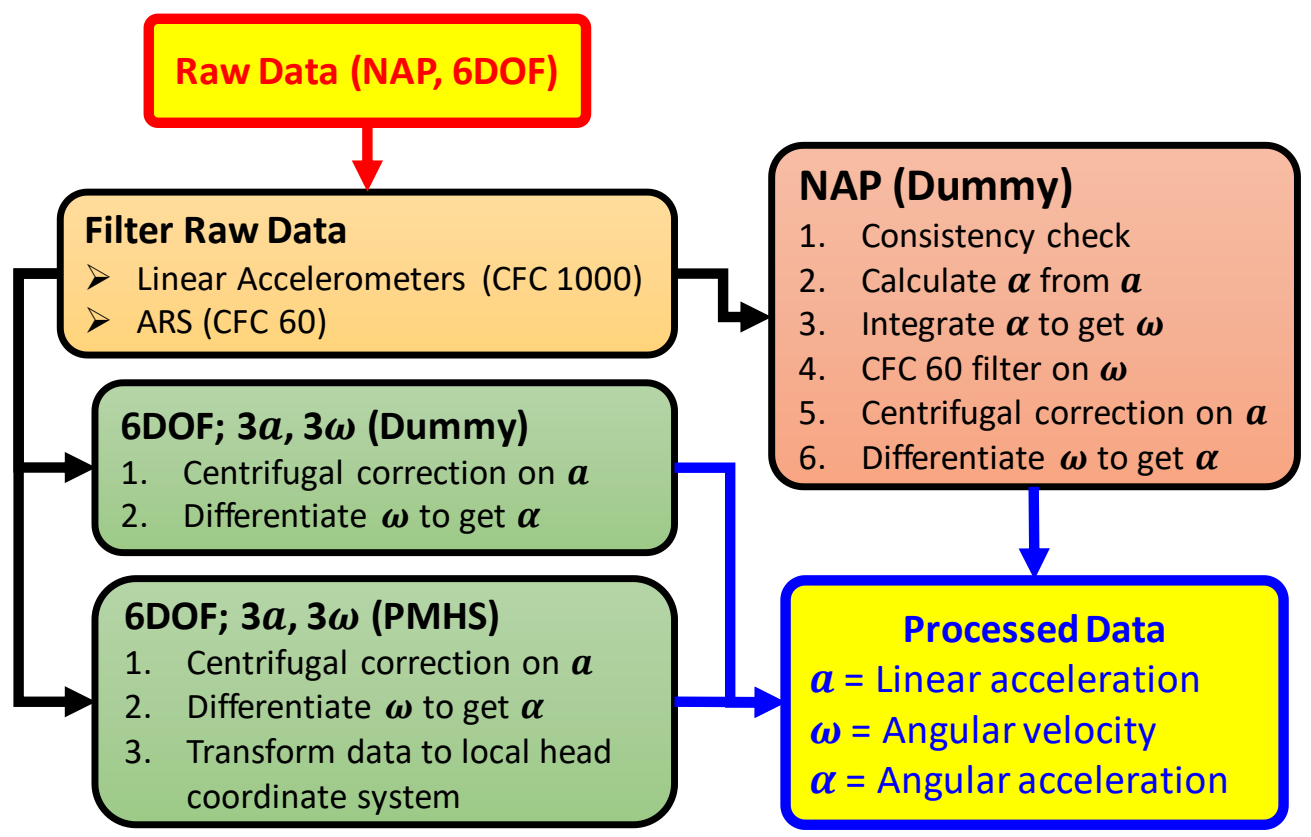

(B)

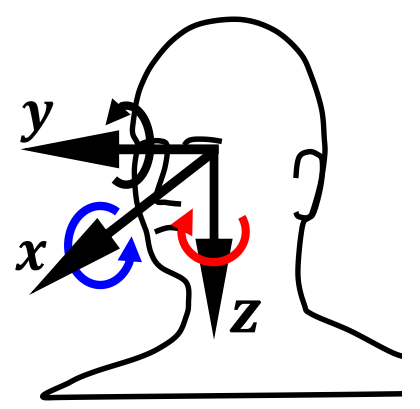

Figure 2-2: Work flow for processing head kinematics data from the raw sensor measurements (A), and the SAE-J211 local head coordinate system (B). Data from cadavers were processed under Post Mortem Human Surrogates (PMHS) .

Head kinematics measured from dummies with internally-mounted sensor packages; Nine Accelerometer Package (NAP) and Angular Rate Sensors (ARS) were 
processed using established techniques (Padgaonkar et al., 1975; Takhounts et al., 2009). Data from externally mounted sensor packages involving human cadavers underwent rigid body and coordinate system transformations to obtain kinematic measurements in the local head coordinate system (Robbins, 1983). Linear accelerations and angular velocities were filtered at channel frequency class (CFC) $1000(1650 \mathrm{~Hz})$ and CFC $60(100 \mathrm{~Hz})$, respectively using a zero-phase shift, digital 8 pole IIR Butterworth filter (NHTSA, 2015). Angular accelerations were obtained by differentiating the filtered angular velocity data using a first order method. Additional details on the techniques used to transform the data to the local head coordinate system are included in Appendix A. Details on scaling the $5^{\text {th }}$ percentile female head kinematics to a $50^{\text {th }}$ male are provided in Appendix B. Distributions for the maximum resultant kinematic parameters are shown in Figure 2-3.
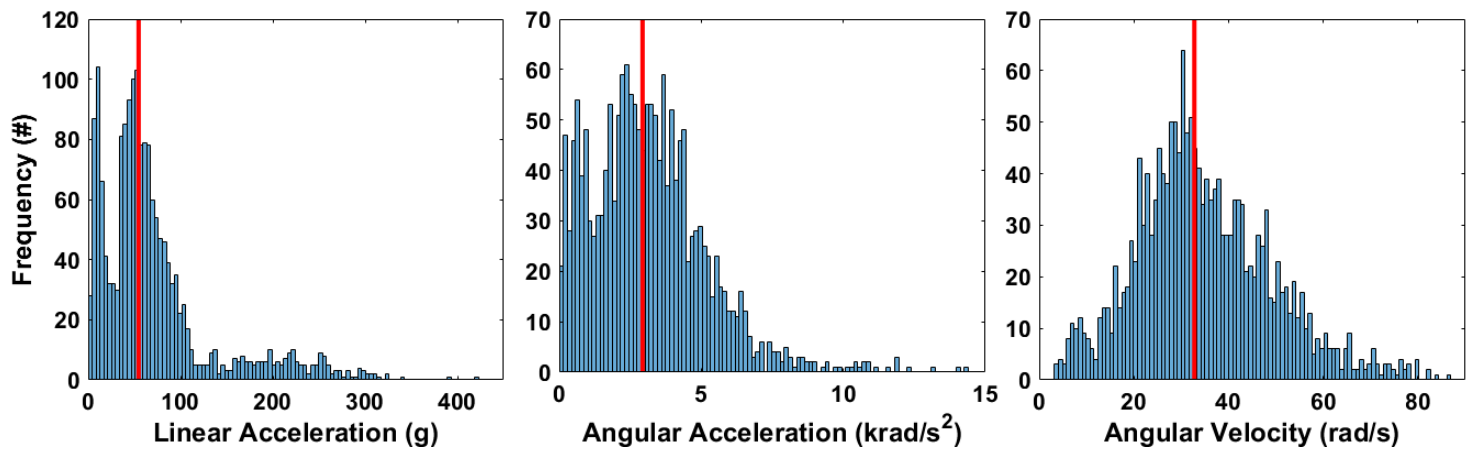

Figure 2-3: Distribution of maximum resultant linear acceleration (left), angular acceleration (middle), and angular velocity (right) for the database $(n=1747)$.

Solid red lines indicate the median values from each distribution: $55 \mathrm{~g}, 2.9$ $\mathrm{krad} / \mathrm{s}^{2}$, and $33 \mathrm{rad} / \mathrm{s}$, respectively.

Brain strain sensitivity to head kinematic frequency content was investigated prior to FE model simulation of the database to determine an appropriate low pass filter. Filter frequencies for ten head impacts that included different severities, sensor types, and surrogates were varied and simulated in the FE brain models. Negligible attenuation of 
brain strain was observed for angular velocity signals filtered down to CFC 60; specification for BrIC, which was then used for all head impacts prior to FE model simulations. Results for the filter sensitivity study are provided in Appendix C.

\subsection{Finite Element Modeling}

\subsubsection{FE Brain Models}

Two different FE models of the human head were used in this dissertation: The Global Human Body Models Consortium-owned (GHBMC) 50th percentile male (M50) detailed seated occupant (v4.3) head model and the Simulated Injury Monitor (SIMon, v4.0), a 50th percentile male human head model developed and distributed by NHTSA (Figure 2-4). The SIMon model is a brain-skull-only model, while the GHBMC head was extracted from the whole body and simulated independently. Both models consist of major brain structures including the cerebrum, cerebellum, and brain stem, and have been used previously in a number of computational brain injury research studies, e.g., (Ji et al., 2014; Mao et al., 2013; Miller et al., 2017; Takhounts et al., 2008). The brain parts were modeled as Kelvin-Maxwell viscoelastic materials, while membranes were modeled as elastic materials. The skull was modeled as a rigid structure, which allowed for direct application of the 6DOF head kinematics in the SAE-J211 local head coordinate system. Since the skull $(\mathrm{GPa})$ is much stiffer than the brain $(\mathrm{kPa})$, it will have negligible deformation in most closed-head inertial impacts, thus it reasonable to assume the skull behaves as a rigid body.

The GHBMC brain model consists of 65,628 elements with an average \pm S.D. volume of $16.7 \pm 12.5 \mathrm{~mm}^{3}$ and runs 2.3 times longer than the SIMon model which has a coarser brain mesh totaling 30,130 elements with an average volume of $34.7 \pm 30.1 \mathrm{~mm}^{3}$. Relative to SIMon, GHBMC is more anatomically detailed and consists of several 
additional substructures including the corpus callosum, thalamus, basal ganglia, and midbrain. The GHBMC also distinguishes between white and gray matter regions, and the layers of meninges, whereas SIMon does not. Both models have been validated for various intracranial responses to head impact including relative brain skull motion (Hardy et al., 2001, 2007) and intracranial pressure (Nahum et al., 1977; Trosseille et al., 1992). This dissertation focuses primarily on results from the GHBMC head model, since it is more detailed; SIMon was used to verify results from GHBMC. A summary of the material properties and brain parts used for both FE models is provided in Appendix D.

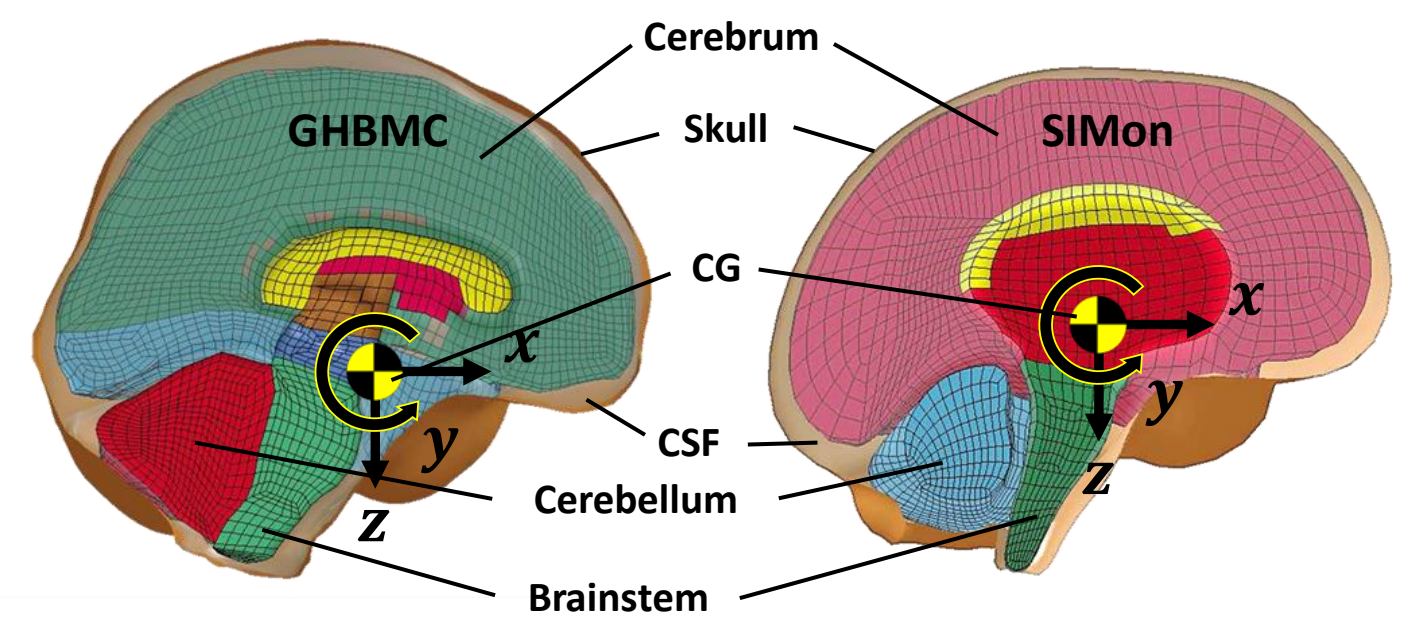

Figure 2-4: Mid sagittal plane view of the GHBMC (left) and SIMon (right) FE brain models, and their respective local head coordinate systems. The SIMon local coordinate system was setup by NHTSA, while the GHBMC local coordinate system was verified for consistency with the kinematic measurements using anatomical landmarks from the skull. Head kinematics were applied to the dura (Skull) in GHBMC, since its nodes were originally connected to the inner skull.

\subsubsection{FE Head Impact Simulations}

Head impact simulations were performed by prescribing the processed 6DOF head kinematics; directionally dependent linear acceleration and annular velocity time histories, directly to the rigid skull of the FE models in the local head coordinate system. The time 
history of brain tissue response was calculated and maximum principal strains were extracted for each element from the simulation output (Figure 2-5). All FE simulations were performed using LS-DYNA (LSTC, v971 R7.1.1, double precision). Computation times were 1.05 and 0.45 minutes of real time per millisecond of simulation time for GHBMC and SIMon, respectively, with parallel processing on a 12-core Linux machine (Dual AMD Opteron $3.2 \mathrm{GHz}, 64 \mathrm{~GB}$ ).
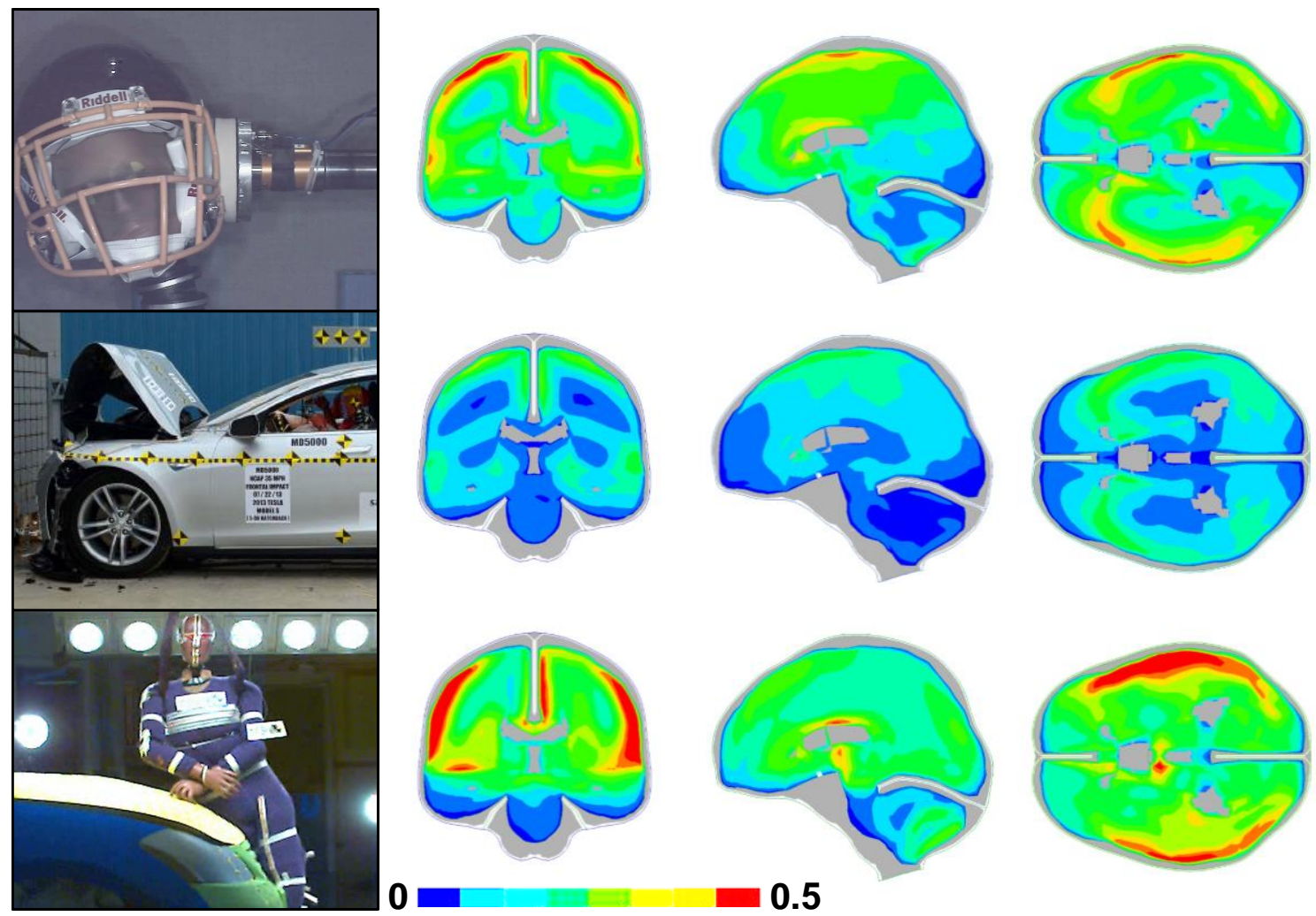

Figure 2-5: Maximum principal strain distributions from GHBMC for several example head impacts. Brain cross sections were taken from the coronal (left column), sagittal (middle column), and axial (right column) planes. Void (gray) regions represent CSF, membranes, and ventricles, which are not shown, and not included in the calculation of the strain-based metrics. Bottom left image was adapted from Sanchez et al., 2017.

\subsubsection{Strain-based Metrics}

Two separate strain-based brain injury metrics were calculated from the FE model simulation outputs: Maximum Principal Strain (MPS) and Cumulative Strain Damage 
Measure (CSDM). Both metrics were developed in previous studies, e.g., (Bandak, 1995; Kleiven, 2007; Takhounts et al., 2003b, 2008), and are considered to be tissue-level predictors of diffuse brain injury. In addition, both metrics have been used in the development of brain injury risk functions for humans based on either football reconstructions (Kleiven, 2007) or scaled animal injury data (Takhounts et al., 2013).

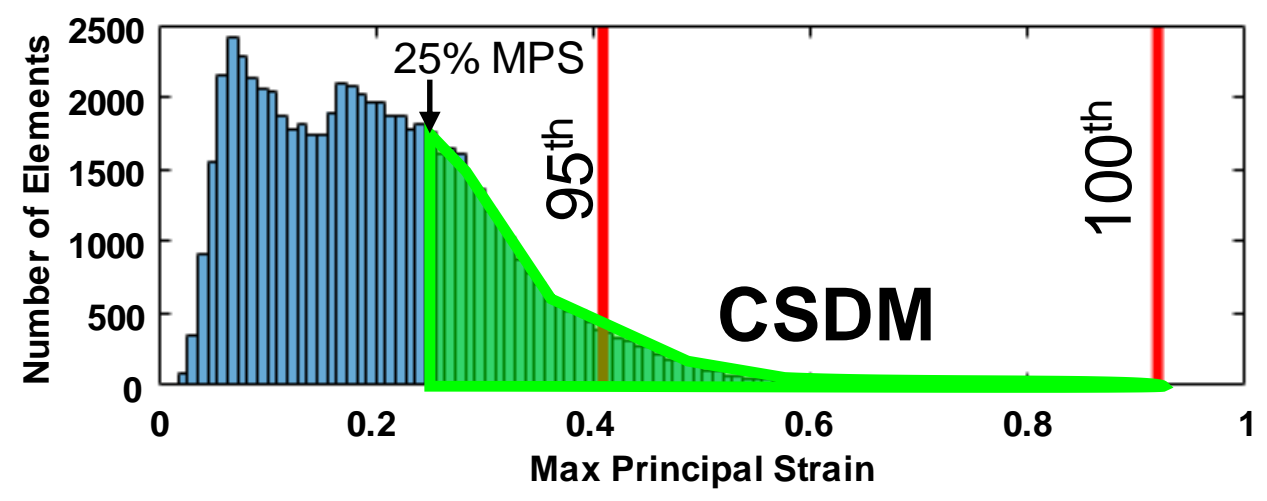

Figure 2-6: Distribution of the time history maximum element-wise MPS from all brain elements for a single head impact. The $9^{\text {th }}$ and $100^{\text {th }}$ percentile element MPS are shown by the solid red lines. The green highlighted region indicates the elements that have achieved a MPS $\geq 25 \%$ during the impact. These elements are considered to be damaged, and are included in the CSDM calculation.

MPS is calculated as the maximum value of maximum principal strain occurring over all elements in the brain, and over the entire impact time history (Takhounts et al., 2008). Many studies have used MPS based on the maximum element value (Kleiven, 2007; Takhounts et al., 2013; Yanaoka et al., 2015). For this dissertation, the $95^{\text {th }}$ percentile value (ranked by element) was used as a surrogate for maximum brain strain to avoid instabilities that may arise with the maximum element (Panzer et al., 2012) (Figure 2-6). The 50 percentile value (ranked by element) was also used. While $95^{\text {th }}$ percentile MPS may be a better indicator of focal brain injury types, the $50^{\text {th }}$ percentile value, being an average measure of brain deformation, may be more appropriate for assessing diffuse brain injuries. 
CSDM is the cumulative volume fraction of elements that incur maximum principal strain that exceeds a predefined threshold value. A threshold of $25 \%$ element-wise MPS was used for CSDM, since it was recently shown to be the best indicator of DAI based on survival analysis using scaled animal data in SIMon and GHBMC (Takhounts et al., 2013) (Figure 2-6). While the results for this dissertation focus on a 25\% threshold for CSDM, additional thresholds, e.g. $20 \%$, and $30 \%$ were calculated to check consistency of the results. Further information regarding the mathematical calculation of CSDM can be found in Appendix E. Distributions for strain-based metrics from the FE model simulations of the head impact database are shown in Figure 2-7.
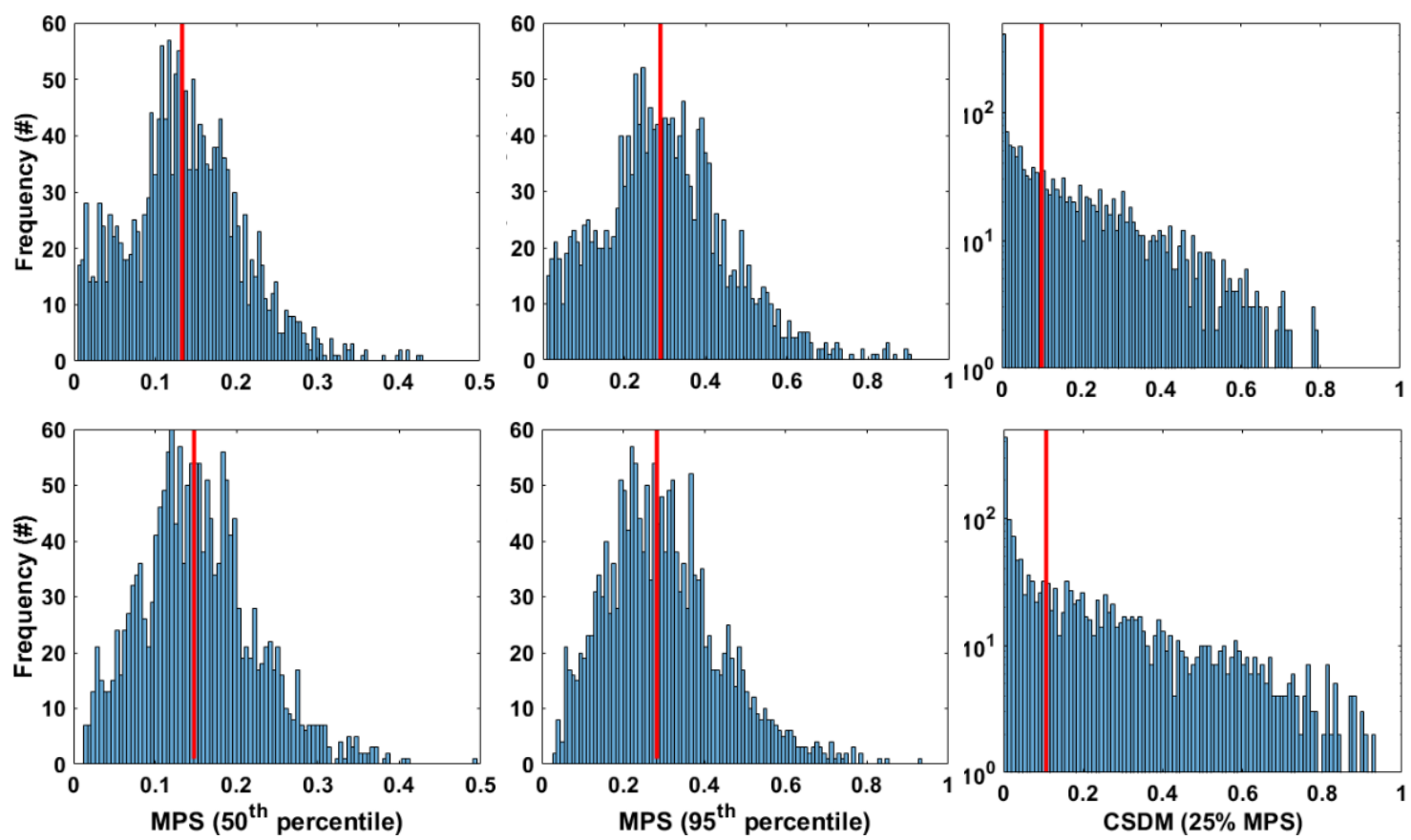

Figure 2-7: Distribution of strain-based metrics for GHBMC (top row) and SIMon (bottom row); MPS 50 ${ }^{\text {th }}$ percentile (left), MPS 95 ${ }^{\text {th }}$ percentile (middle), and CSDM (25\% MPS) (right) for the database $(n=1747)$. Solid red lines indicate the median values from each distribution (GHBMC; SIMon): $(0.13 ; 0.15),(0.29$;

$0.28)$, and $(0.10 ; 0.11)$, respectively. 


\section{Chapter 3 - Assessment of Kinematic-based Metrics}

Numerous injury criteria have been developed to predict brain injury using the kinematic response of the head during impact. Each criterion utilizes a metric that is some mathematical combination of the velocity and/or acceleration components of translational and/or rotational head motion. Despite the large number of metrics that have been proposed, no single metric has been universally accepted for a broad range of head impacts. In this chapter, existing kinematic-based metrics are assessed for their capability to predict strain-based responses from the FE brain models. The objective of this chapter is to identify key head kinematic parameters for predicting brain strain in various impact conditions. This chapter was published previously (Gabler et al., 2016. Assessment of kinematic brain injury metrics for predicting strain responses in diverse automotive impact conditions. Annals of Biomedical Engineering, 44(12), 3705-3718; DOI: 10.1007/s10439-016-16970 ), and was adapted for this dissertation with permission from the publisher.

\subsection{Introduction}

Brain injury risk assessments have typically focused on the resulting kinematics of the head during impact. Traditionally, these assessments have been achieved through an evaluation of resultant linear head acceleration, e.g., NHTSA 1996; NOCSAE, 2012; and (Rowson and Duma, 2011). However, despite widespread use of translational-based metrics for head injury assessment, many question whether they are suitable for assessing brain injuries, e.g., (Gennarelli et al., 1971; Margulies and Thibault, 1992; Ommaya and Gennarelli, 1974). Considering Holbourn's 1943 hypothesis, which states that brain injuries are governed by the fundamental laws of motion and the physical properties of the brain-skull system, rotational kinematics seem most suitable for assessing brain injuries in 
clos impact events. Although numerous head injury metrics have been proposed, it remains unclear as to which metric should be used for assessing brain injury severity in a particular impact condition.

In this chapter, existing kinematic-based metrics were assessed for their capability to predict strain-based responses from FE model simulation of a diverse dataset of head impacts. A total of fifteen different kinematic-based metrics, including fundamental parameters of motion in addition to metrics from common injury criteria, were evaluated. A subset of head impacts from the database (Section 2.1.1) including sled, crash, and impactor tests were simulated in the FE human head models, and used for the analysis. Assessments were made by comparing correlations between kinematic metrics, which were calculated directly from the head impact data, and strain-based metrics, which were

obtained through the FE model simulations. These results identify best performing kinematic-correlates for the prediction of brain tissue strains, and highlight instances where certain injury criteria may not be suitable.

\subsection{Methods}

\subsubsection{Head Impact Data}

Timed 6DOF head kinematics for 660 cases from the database (Section 2.1.1) were used in this assessment. These impacts include sled tests from UVa, crash and pendulum impactor tests from NHTSA, and crash tests from IIHS. A summary of the impact conditions included in this chapter is shown in Table 3-1, and a detailed list of the head impacts used is provided in Appendix I. All head kinematic data were processed and prepared for calculation of kinematic and strain-based injury metrics according to the methods described in Chapter 2 and Appendix A. 
Table 3-1: Summary of the impact conditions used in Chapter 3

\begin{tabular}{cc}
\hline $\begin{array}{c}\text { Impact condition } \\
\text { (sample size) }\end{array}$ & Test type (sample size) \\
\hline $\begin{array}{c}\text { Frontal (FRT) } \\
(325)\end{array}$ & UVa driver side universal gold standard sled tests (13) \\
& NHTSA NCAP/RD driver \& front pass. crash tests (87) \\
NHTSA $0^{\circ}$ pendulum into head CG/Forehead (74) & IIHS small \& moderate overlap impact driver crash tests (151) \\
\hline $\begin{array}{c}\text { Oblique (OBL) } \\
(163)\end{array}$ & UVa $20^{\circ} / 60^{\circ}$ far side oblique sled tests (31) \\
& NHTSA RD driver \& rear pass. near side crash tests (54) \\
NHTSA $30^{\circ} / 60^{\circ}$ pendulum into head CG/Forehead (78)
\end{tabular}

\subsubsection{Kinematic-based Metrics}

Fifteen kinematic-based head and brain injury metrics were evaluated in this study. These metrics were selected based on their inclusion of a variety of translational and rotational head kinematic parameters consisting of linear and angular velocities and accelerations. Most metrics were formulated based on empirical fits using experimental datasets, while others were developed using fundamental mechanics theories or computational approaches. Several of these metrics have been used in the development of head and brain injury risk functions. A brief description of each metric is provided below and summarized in Table 3-2. Head kinematics are described by the directionally dependent, time history vectors of linear acceleration, $\boldsymbol{a}(t)$, angular acceleration, $\boldsymbol{\alpha}(t)$, and angular velocity, $\boldsymbol{\omega}(t)$. Subscripts in the equations below indicate the different directional components $(x, y, z)$ and critical values (cr), which are specified in Table 3-2. 
Maximum resultant acceleration and velocity: The most fundamental kinematic parameters used in head and brain injury risk assessments are the maximum values of the resultant directionally dependent head angular velocity, and linear and angular acceleration time histories. They are defined as follows:

$z_{m}=\max _{t}\{|\boldsymbol{z}(t)|\}$,

Equation 3-1

where $\mathbf{z}$ is equal to $\boldsymbol{a}, \boldsymbol{\alpha}$, or, $\boldsymbol{\omega}$, and $z_{m}$ is maximum value of the resultant kinematic variable taken over the entire time history of the event.

Gadd Severity Index (GSI): In 1966, Gadd proposed the severity index as a means of incorporating impact duration into the assessment of head and brain injury potential (Gadd, 1966). The GSI was formulated to approximate the Wayne State Tolerance Curve (WSTC); an experimentally derived threshold for skull fracture based on an inverse relationship between linear head acceleration and impact duration (Patrick et al., 1963): $G S I=\int|\boldsymbol{a}(t)|^{2.5} d t$,

Equation 3-2

where the acceleration units are (g). The GSI was calculated according to NOCSAE standards, where the integral is evaluated from when $|\boldsymbol{a}(t)|$ first rises above $4 \mathrm{~g}$ to when it falls below 4g following the maximum value (NOCSAE 2012).

Head Injury Criterion (HIC): In 1971, Versace adapted the GSI to include a timeaveraging component, which became known as HIC. HIC is the most commonly used metric for evaluating head injury risk, and is currently the only federally-mandated head injury assessment used in motor vehicle regulation (Department of Transportation NHTSA Docket Number 69-7, Notice 19).

$H I C=\max _{\left(t_{1}, t_{2}\right)}\left\{\left(t_{2}-t_{1}\right)\left[\frac{1}{t_{2}-t_{1}} \int_{t_{1}}^{t_{2}}|\boldsymbol{a}(t)| d t\right]^{2.5}\right\}$,

Equation 3-3 
where, the units of acceleration are $(\mathrm{g})$ and $t_{1}, t_{2}$ are selected from the time domain in (s) such that the value of HIC is maximized. Based on current standards, $t_{2}-t_{1} \leq 15 \mathrm{~ms}$.

Generalized Acceleration Model for Brain Injury Threshold(GAMBIT): One of the earliest efforts to combine translational and rotational kinematics for brain injury risk assessment was the development of GAMBIT (Newman, 1986). GAMBIT is based on a kinematic analog to maximum shear stress/strain theory, where the relationship between linear and angular head acceleration is assumed analogous to that of the normal and shear components of maximum in-plane shear, respectively.

$G A M B I T=\max _{t}\left\{\left[\left(\frac{|\boldsymbol{a}(t)|}{a_{c r}}\right)^{n}+\left(\frac{|\boldsymbol{\alpha}(t)|}{\alpha_{c r}}\right)^{m}\right]^{1 / s}\right\}$

Equation 3-4

where the exponents, $n, m, s$ are empirically determined constants that were set to two based on the original formulation of the metric.

Head Impact Power (HIP): In 2000, Newman et al. proposed another combined kinematic brain injury metric, HIP, which is based on the hypothesis that head injury severity correlates with maximum head impact power (Newman et al., 2000). The formulation for HIP is based on the rate of change of kinetic energy entering a rigid body with mass and inertia properties based on a $50^{\text {th }}$ percentile adult, human male:

$H I P=\max _{t}\left\{m \sum a_{i}(t) \int a_{i}(t) d t+\sum I_{i i} \alpha_{i}(t) \int \alpha_{i}(t) d t\right\}$

Equation 3-5

where $I_{i i}$ are the principal moments of inertia of the head, with mass $m$, about the anatomical axes $(i=x, y, z)$.

Kleiven's Linear Combination (KLC): In 2007, Kleiven et al. found that a linear combination of HIC and $\omega_{m}$ correlated well with brain MPS obtained from FE simulation of head impacts sustained by American professional football players (Kleiven, 2007). 
$K L C=\beta_{1} \omega_{m}+\beta_{2} H I C_{36}$,

Equation 3-6

where $\beta_{i}$ are the regression coefficients, and the subscript on HIC represents the constraint that $t_{2}-t_{1} \leq 36 \mathrm{~ms}$.

Principal Component Score (PCS): Another empirically derived model used for concussion assessment in professional football players was developed by Greenwald et al. using principal component analysis (Greenwald et al., 2008). This metric is a linear combination of several kinematic metrics mentioned above:

$P C S=\beta_{o}+\beta_{1} \overline{a_{m}}+\beta_{2} \overline{G S I}+\beta_{3} \overline{H I C_{15}}+\beta_{4} \overline{\alpha_{m}}$,

Equation 3-7

where $\bar{X}=(X-\mu) / \sigma$ is the standardized value for each kinematic metric. Values for $\mu$ and $\sigma$ were based on the 660 head impacts used in this chapter.

Combined Probability of Concussion (CP): CP was developed by Rowson et al., 2013 to predict concussion risk experienced by collegiate football players following helmet impact (Rowson and Duma, 2013). The actual kinematic metric is the basis of a logistic function taken from an injury risk curve that relates maximum resultant linear and angular head acceleration to concussion risk,

$C P=\beta_{0}+\beta_{1} a_{m}+\beta_{2} \alpha_{m}+\beta_{3} a_{m} \alpha_{m}$,

Equation 3-8 where $\beta_{i}$ were obtained from a multivariate logistic regression analysis.

Power Rotational Head Injury Criterion (PRHIC): Beginning in 2011, Kimpara et al., began modifying HIC with rotational kinematic parameters (Kimpara et al., 2011). The first of two proposed rotational brain injury criteria from this group, PRHIC was developed by replacing the linear acceleration term of HIC with the rotational component of HIP.

PRHIC $=\max _{\left(t_{1}, t_{2}\right)}\left\{\left(t_{2}-t_{1}\right)\left[\frac{1}{t_{2}-t_{1}} \int_{t_{1}}^{t_{2}}\left|H I P_{\text {rot }}(t)\right| d t\right]^{2.5}\right\}$,

Equation 3-9

where, 
$H I P_{\text {rot }}(t)=\sum I_{i i} \alpha_{i}(t) \int \alpha_{i}(t) d t$

Equation 3-10

Rotational Injury Criterion (RIC): The second rotational brain injury metric proposed by Kimpara et al., 2012, was RIC (Kimpara and Iwamoto, 2012). RIC is formulated in a similar manner to PRHIC, but with the linear acceleration term in HIC replaced with angular acceleration in $\left(\mathrm{rad} / \mathrm{s}^{2}\right)$.

$R I C=\max _{\left(t_{1}, t_{2}\right)}\left\{\left(t_{2}-t_{1}\right)\left[\frac{1}{t_{2}-t_{1}} \int_{t_{1}}^{t_{2}}|\alpha(t)| d t\right]^{2.5}\right\}$,

Equation 3-11 where a constraint of $t_{2}-t_{1} \leq 36 \mathrm{~ms}$ was proposed for both PRHIC and RIC.

Kinematic Rotational Brain Injury Criterion (BRIC): BRIC was proposed by Takhounts et al., 2011 to assess brain injury risk due to DAI in occupant crash tests that otherwise may not have been detected by HIC (Takhounts et al., 2011). A preliminary form of the metric was proposed in 2011 and included angular velocity and acceleration:

$B R I C=\frac{\omega_{m}}{\omega_{c r}}+\frac{\alpha_{m}}{\alpha_{c r}}$.

Equation 3-12

Brain Injury Criterion (BrIC): In 2013, Takhounts et al., proposed an update to the original BRIC formulation after finding that angular velocity alone was sufficient to predict FE model strains in pendulum and several occupant crash test modes (Takhounts et al., 2013). The updated metric, BrIC, is formulated using the maximum magnitudes of the three orthogonal head angular velocity components:

$B r I C=\sqrt{\left(\frac{\omega_{x}}{\omega_{x c r}}\right)^{2}+\left(\frac{\omega_{y}}{\omega_{y c r}}\right)^{2}+\left(\frac{\omega_{z}}{\omega_{z c r}}\right)^{2}}$,

Equation 3-13

where $\omega_{i c r}$ are directionally dependent critical values that were determined using the SIMon FE model.

Rotational Velocity Change Index (RVCI): In 2015, Yanaoka et al. proposed RVCI to improve correlations between head kinematics and FE model strains for pedestrian 
impacts (Yanaoka et al., 2015). RVCI was formulated by assuming that brain strain was analogous to deformation from a simple spring mass model:

$R V C I=\max _{\left(t_{1}, t_{2}\right)} \sqrt{R_{x}\left(\int_{t_{1}}^{t_{2}} \alpha_{x} d t\right)^{2}+R_{y}\left(\int_{t_{1}}^{t_{2}} \alpha_{y} d t\right)^{2}+R_{z}\left(\int_{t_{1}}^{t_{2}} \alpha_{z} d t\right)^{2}}, \quad$ Equation 3-14 where $R_{i}$ are weighting factors about each orthogonal axis determined using the GHBMC FE model. A duration constraint of $t_{2}-t_{1} \leq 10 \mathrm{~ms}$ was imposed since it resulted in the highest correlation with strain-based metrics for occupant and pedestrian crash simulations.

\subsubsection{FE Model Simulations and Statistics}

MPS and CSDM were calculated for each of the 660 head impacts by the applying 6DOF kinematics to the GHBMC and SIMon FE head models (Section 2.2). Simple linear regression using ordinary least squares was used to assess the capability of each kinematicbased metric to predict MPS and CSDM:

$Y_{j}=\beta_{0}+\beta_{1} X_{j}+\epsilon_{j}$

Equation 3-15

where $Y_{j}$ is the $j^{\text {th }}$ strain-based outcome for a particular kinematic-based metric, $X_{j}$, and residual (error), $\epsilon_{j}$. Regression coefficients include the intercept, $\beta_{0}$, and regressor, $\beta_{1}$.

An overall assessment was made using the 660 head impacts and simulation results from both FE models. In an additional assessment, head impacts were categorized into four separate impact conditions: frontal, oblique, side, and pedestrian (Table 3-1). Kinematicto-strain-metric correlations were assessed using the coefficient of determination $\left(\mathrm{R}^{2}\right)$ (Milton and Arnold, 2002):

$R^{2}=\frac{\left[\sum_{j=1}^{n}\left(X_{j}-\bar{X}\right)\left(Y_{j}-\bar{Y}\right)\right]^{2}}{\sum_{j=1}^{n}\left(X_{j}-\bar{X}\right)^{2} \cdot \sum_{j=1}^{n}\left(Y_{j}-\bar{Y}\right)^{2}}$

where $n$ is the sample size. Test for significance was evaluated at the 5\% level $(\alpha=0.05)$. 
Table 3-2: Existing kinematic-based injury metrics.

\begin{tabular}{|c|c|c|c|c|}
\hline Metric & Type & $\begin{array}{c}\text { Primary } \\
\text { method of } \\
\text { development }\end{array}$ & $\begin{array}{l}\text { Injury } \\
\text { assessment } \\
\text { type }\end{array}$ & Constraints and critical values \\
\hline$G S I$ & $\mathrm{~T}$ & Experimental & $\begin{array}{l}\text { Skull } \\
\text { Fracture, } \\
\text { TBI }\end{array}$ & $\begin{array}{l}\text { 4g threshold } \\
\text { (Equation 3-2) }\end{array}$ \\
\hline HIC & $\mathrm{T}$ & Experimental & $\begin{array}{l}\text { Skull } \\
\text { Fracture, } \\
\text { TBI }\end{array}$ & $\begin{array}{c}t_{2}-t_{1} \leq 15 \mathrm{~ms} \\
\text { (Equation 3-3) }\end{array}$ \\
\hline GAMBIT & $\mathrm{T}, \mathrm{R}$ & Theoretical & Concussion & $a_{c r}=\underset{(\text { Equation 3-4) }}{250 \mathrm{~g}, \alpha_{c r}=25 \mathrm{krad} / \mathrm{s}^{2}}$ \\
\hline$H I P$ & $\mathrm{~T}^{\mathrm{c}}, \mathrm{R}^{\mathrm{c}}$ & Theoretical & Concussion & $\begin{array}{c}m=4.5 \mathrm{~kg}, I_{x x}=1.6 \mathrm{E}-2 \mathrm{Nms}^{2}, I_{y y} \\
=2.4 \mathrm{E}-2 \mathrm{Nms}^{2}, I_{z z}=2.2 \mathrm{E}-2 \mathrm{Nms}^{2} \\
(\text { Equation } 3-5)^{2}\end{array}$ \\
\hline$K L C$ & $\mathrm{~T}, \mathrm{R}$ & Computational & Concussion & $\begin{array}{c}\beta_{1}=4.72 \mathrm{E}-3 \mathrm{rad}^{-1} \mathrm{~s} \\
\beta_{2}=2.24 \mathrm{E}-4, t_{2}-t_{1} \leq 36 \mathrm{~ms} \\
\text { (Equation } 3-6)\end{array}$ \\
\hline$P C S$ & $\mathrm{~T}, \mathrm{R}$ & Experimental & Concussion & $\begin{array}{c}\beta_{o}=20, \beta_{1}=4.336 \mathrm{~g}^{-1} \\
\beta_{2}=4.718, \beta_{3}=4.742 \\
\beta_{4}=2.164 \mathrm{rad}^{-1} \mathrm{~s}^{2} \\
\text { (Equation 3-7) }\end{array}$ \\
\hline$C P$ & $\mathrm{~T}, \mathrm{R}$ & Experimental & Concussion & $\begin{array}{c}\beta_{0}=-10.2, \beta_{1}=4.33 \mathrm{E}-2 \mathrm{~g}^{-1}, \\
\beta_{2}=8.73 \mathrm{E}-4 \mathrm{rad}^{-1} \mathrm{~s}^{2}, \\
\beta_{3}=-9.20 \mathrm{E}-7 \mathrm{~g}^{-1} \mathrm{rad}^{-1} \mathrm{~s}^{2} \\
\text { (Equation 3-8) }\end{array}$ \\
\hline $\begin{array}{l}P R H I C, \\
\text { RIC }\end{array}$ & $\mathrm{R}$ & Experimental & Concussion & $\begin{array}{c}t_{2}-t_{1} \leq 3 \mathrm{~ms} \\
\text { (Equations 3-9-3-11) }\end{array}$ \\
\hline BRIC & $\mathrm{R}$ & Computational & DAI & $\begin{array}{c}\mathrm{H}-\mathrm{III}{ }^{\mathrm{a}}: \omega_{c r}=46.41 \mathrm{rad} / \mathrm{s} \\
\alpha_{c r}=39.77 \mathrm{krad} / \mathrm{s}^{2} \\
\mathrm{ES}-2 \mathrm{re}: \omega_{c r}=65.68 \mathrm{rad} / \mathrm{s} \\
\alpha_{c r}=23.06 \mathrm{krad} / \mathrm{s}^{2} \\
\mathrm{WSID}^{\mathrm{b}}: \omega_{c r}=153.18 \mathrm{rad} / \mathrm{s} \\
\alpha_{c r}=11.53 \mathrm{krad} / \mathrm{s}^{2} \\
\text { (Equation } 3-12)\end{array}$ \\
\hline BrIC & $\mathrm{R}^{\mathrm{c}}$ & Computational & DAI & $\begin{array}{c}\omega_{x c r}=66.25 \mathrm{rad} / \mathrm{s}, \omega_{y c r}= \\
56.45 \mathrm{rad} / \mathrm{s}, \omega_{z c r}=42.87 \mathrm{rad} / \mathrm{s} \\
(\text { Equation } 3-13)\end{array}$ \\
\hline$R V C I$ & $\mathrm{R}^{\mathrm{c}}$ & Computational & DAI & $\begin{array}{l}R_{i}=(\mathrm{MPS}, \mathrm{CSDM}): R_{x}=1,1 \\
R_{y}=1,2.29 ; R_{z}=1.17,1.98 \\
\left.t_{2}-t_{1} \leq 10 \mathrm{~ms} \text { (Equation } 3-14\right)\end{array}$ \\
\hline
\end{tabular}

${ }^{a}$ Critical values were used for oblique impacts and tests using the THOR dummy.

${ }^{\mathrm{b}}$ Critical values were used in side and pedestrian impacts and tests using the SID dummy.

${ }^{\mathrm{c}}$ Indicates that the metric is directionally dependent $(i=x, y, z)$.

$\mathrm{T}=$ Translational; $\mathrm{R}=$ Rotational. 


\subsubsection{Isolated Head Motions}

In addition to the assessments above, FE simulations for several head impacts were performed by prescribing separately the rotational and the translational components of the 6DOF kinematics. Head impacts selected for this assessment included some of the highest maximum resultant linear acceleration and HIC values from the database. Strain-based metrics from the isolated head motions were compared to the combined (6DOF) result to isolate the relative contribution of pure rotational and translational head motion on the development of brain strain.

\subsection{Results}

\subsubsection{Overall Assessment}

The assessment using 660 head impacts and GHBMC revealed better correlation between rotational-based kinematic metrics and FE strains when compared to translationalbased metrics (Figure 3-1, A). BrIC was the overall best correlate with MPS $\left(\mathrm{R}^{2}=0.778\right.$; Figure 3-2, A), while RVCI correlated slightly better with CSDM $\left(\mathrm{R}^{2}=0.797\right.$; Figure 3-2, B). Of the combined rotation-translation metrics, which generally correlated better when compared to translation-only metrics, KLC had the highest correlation $\left(\mathrm{R}^{2}=0.402-0.443\right.$; Figure 3-1, A), while GAMBIT had the lowest correlation $\left(\mathrm{R}^{2}=0.032-0.041\right.$; Figure 3-1, A). Kinematic metrics including maximum angular velocity and angular acceleration had similar correlation with both MPS and CSDM $\left(\mathrm{R}^{2}=0.549-0.595\right.$; Figure 3-1, A), while maximum linear acceleration had the lowest overall correlation $\left(\mathrm{R}^{2}=0.017\right.$, Figure $3-2$, C). Correlation between HIC and FE strains was also very low $\left(\mathrm{R}^{2}=0.146\right.$, Figure 3-2, D).

Trends between SIMon and GHBMC were similar; however, correlations between kinematic metrics and FE strains were slightly higher using SIMon (Figure. 3-1, B). Table 
3-3 shows the relative ranking of kinematic-based metrics with respect to $R^{2}$ from correlations with MPS and CSDM. Results from the regression analysis using GHBMC are provided in Table 3-4.

(A) Correlations based on GHBMC

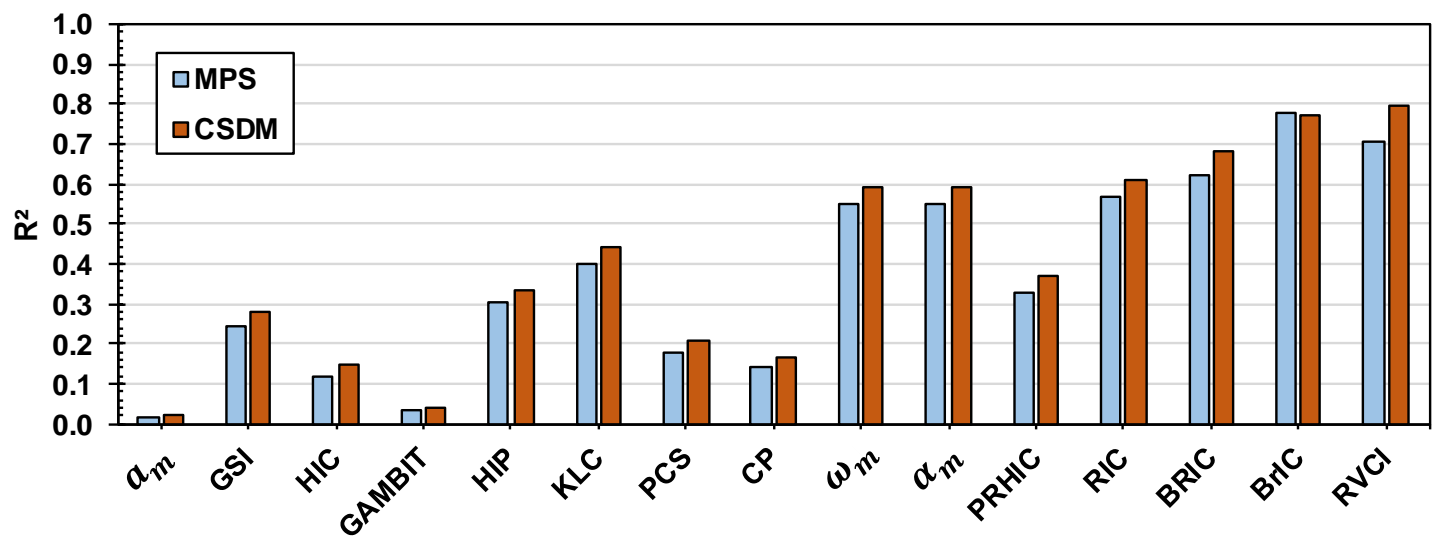

(B) Correlations based on SIMon

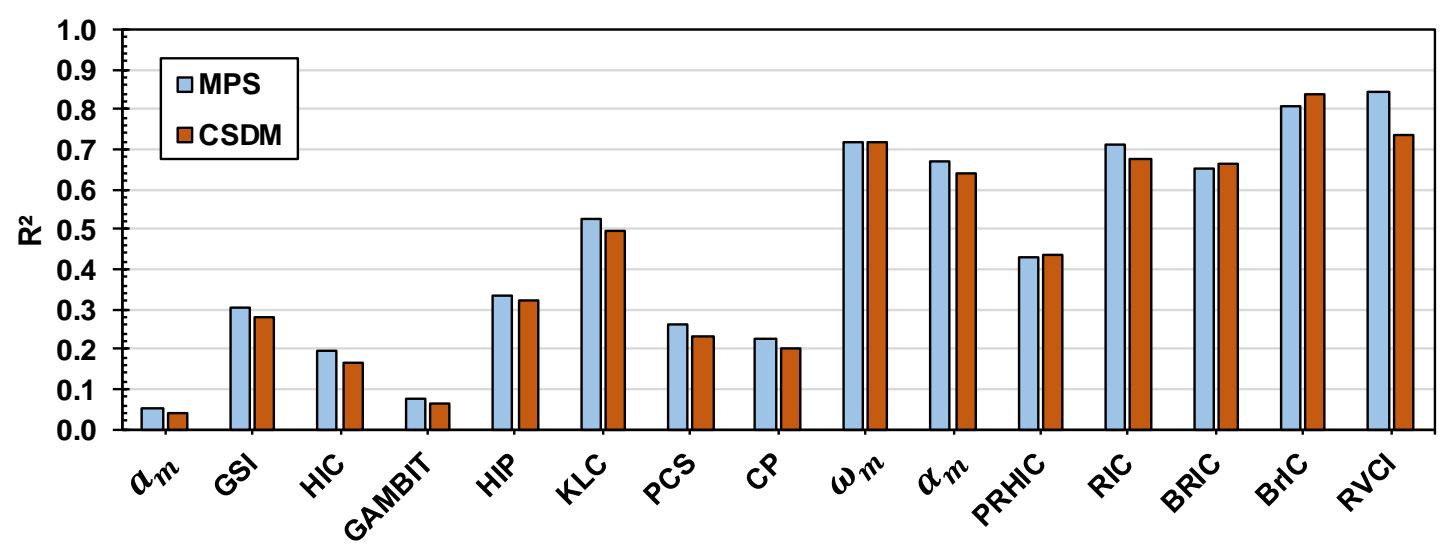

Figure 3-1: Overall correlations between kinematic and strain-based metrics for (A) GHBMC and (B) SIMon. Sample size $n=660$.

\subsubsection{Assessment by Impact Condition}

When separated by impact condition, trends were similar to those observed in the overall assessment. In frontal impacts, BrIC had the highest correlation with FE strains ( $\mathrm{R}^{2}$ $=0.730,0.705)$ for MPS and CSDM, respectively (Figure 3-3, A). In oblique impacts, BrIC had the highest correlation with MPS $\left(\mathrm{R}^{2}=0.793\right)$, while RVCI was highest with CSDM 
$\left(\mathrm{R}^{2}=0.840\right.$; Figure 3-3, B). In side impacts, BrIC had the highest correlation with MPS $\left(R^{2}=0.652\right)$, and RVCI was highest with CSDM $\left(R^{2}=0.739\right.$; Figure 3-3, C). Finally, for pedestrian impacts, RVCI correlated best with both MPS and CSDM $\left(\mathrm{R}^{2}=0.730,0.705\right)$, respectively, followed by RIC (Figure 3-3, D).

(A)

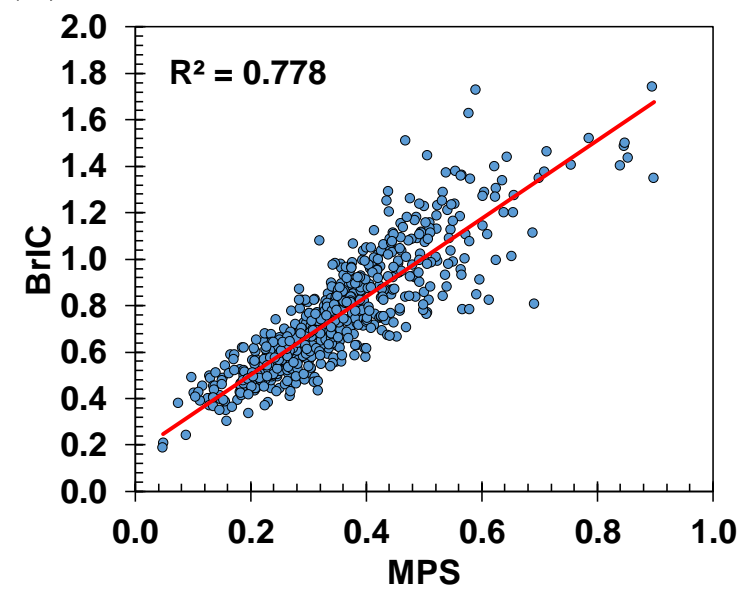

(C)

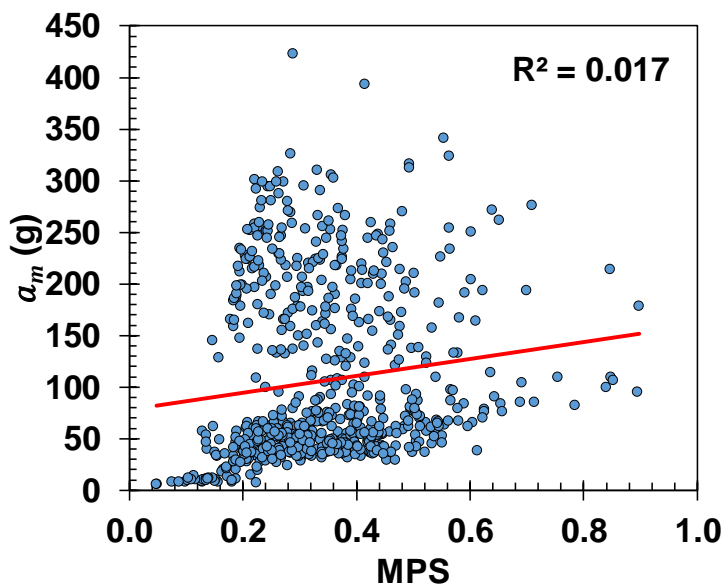

(B)

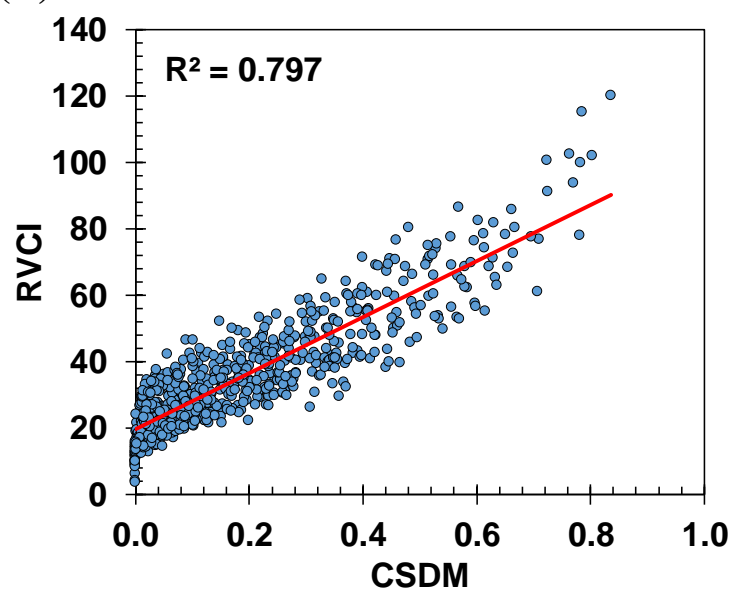

(D)

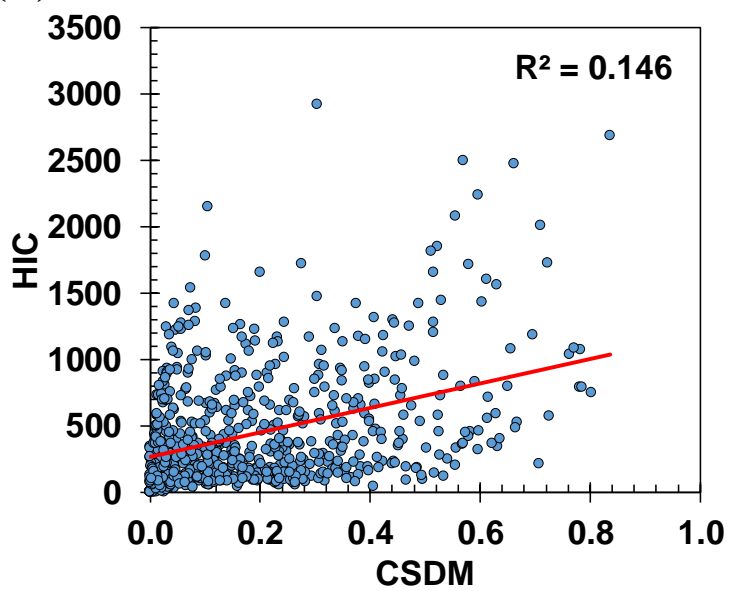

Figure 3-2: Scatter plots showing correlations between strain-based metrics from GHBMC and (A) BrIC, (B) RVCI, (C) $a_{m}$, and (D) HIC. Sample size $n=660$.

\subsubsection{Brain Strain Sensitivity to Isolated Head Motions}

When only the translational components of head kinematics were prescribed for the seven representative cases, brain strains were relatively low and, in some cases, non- 
existent (CSDM). However, when only the rotational components were prescribed, strain responses were nearly identical to those from the combined 6DOF motions (Figure 3-4).

(A) Correlations based on frontal impacts

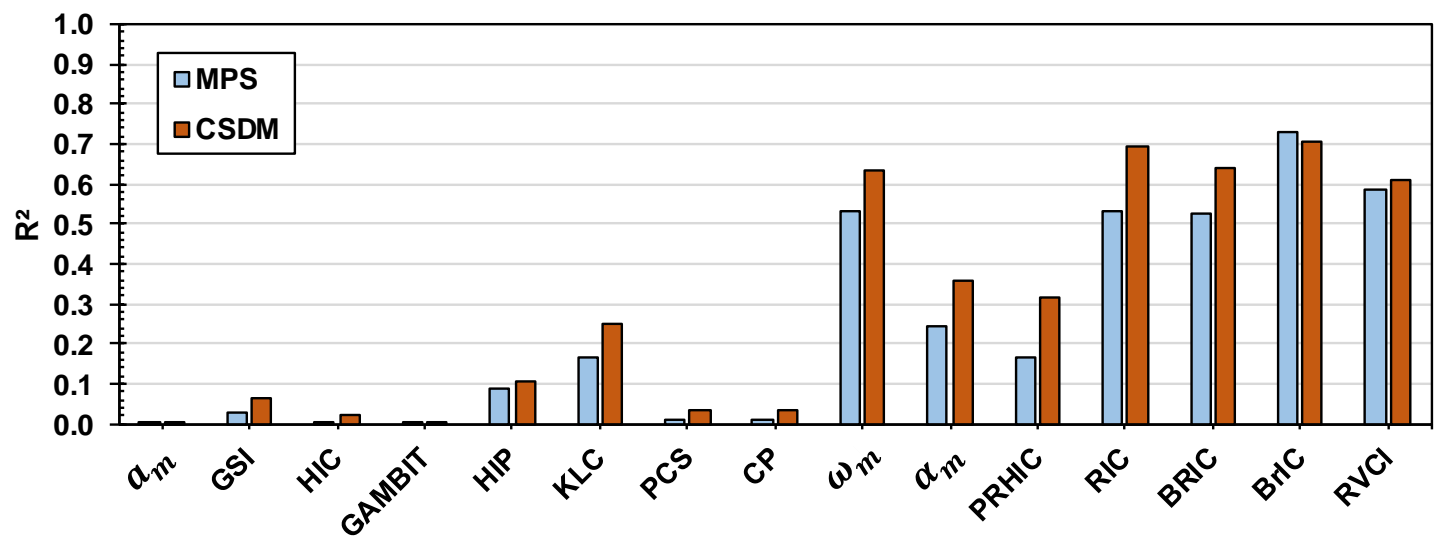

(B) Correlations based on oblique impacts

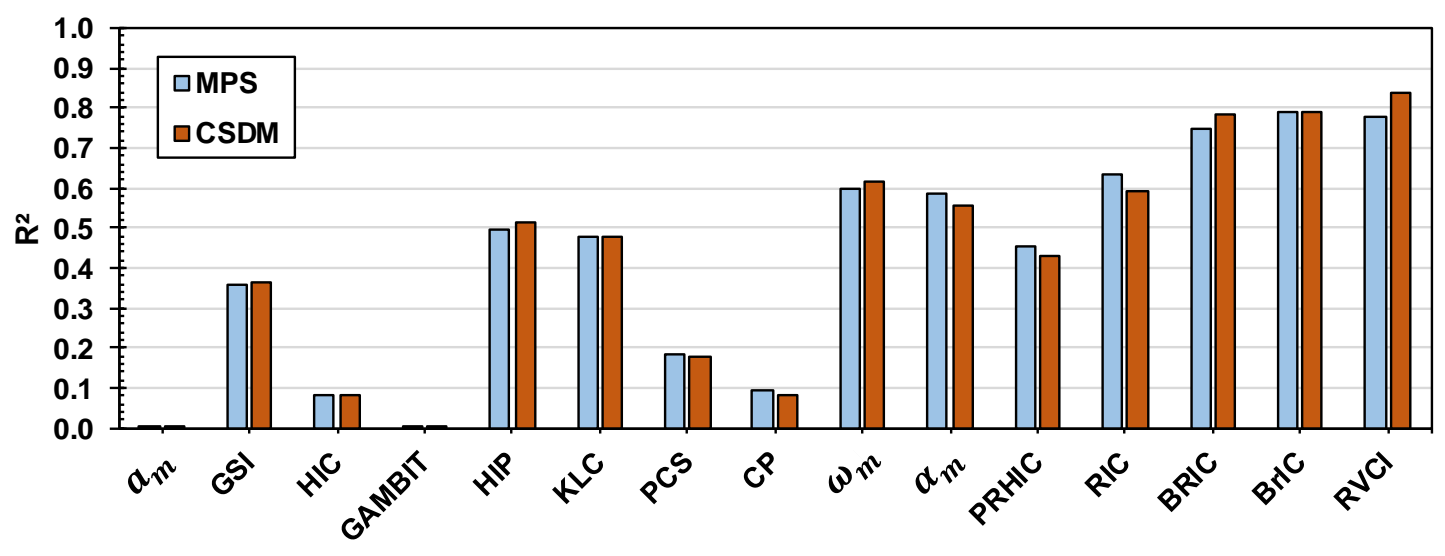

(C) Correlations based on side impacts

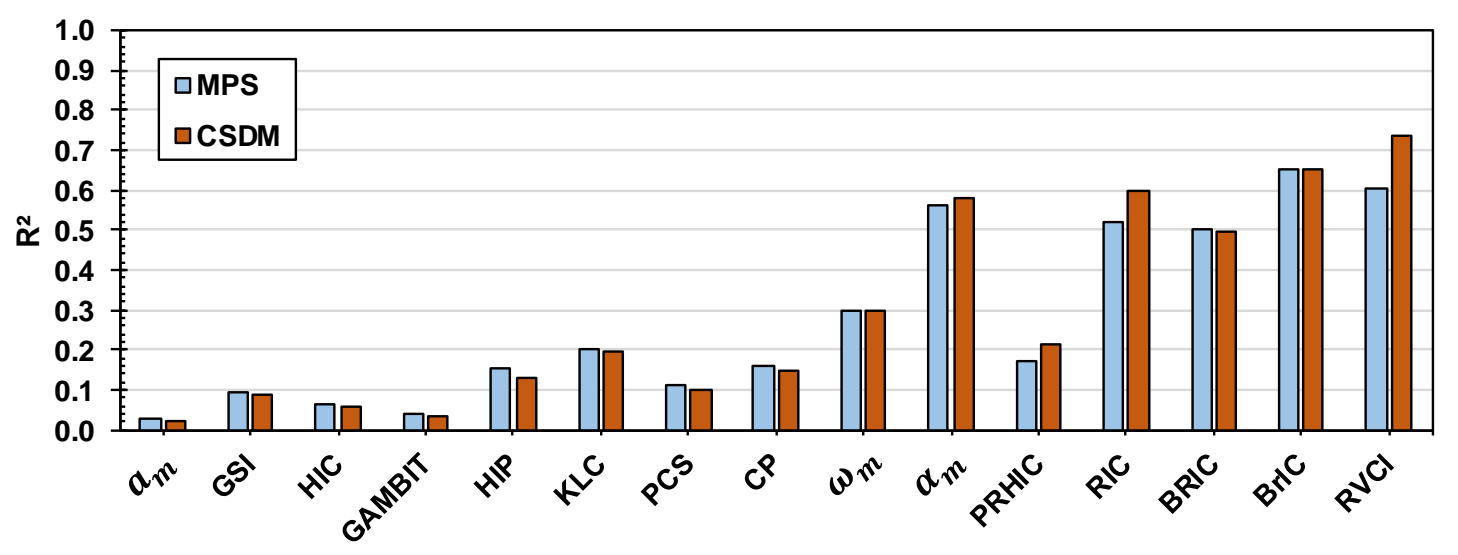


(D) Correlations based on pedestrian impacts

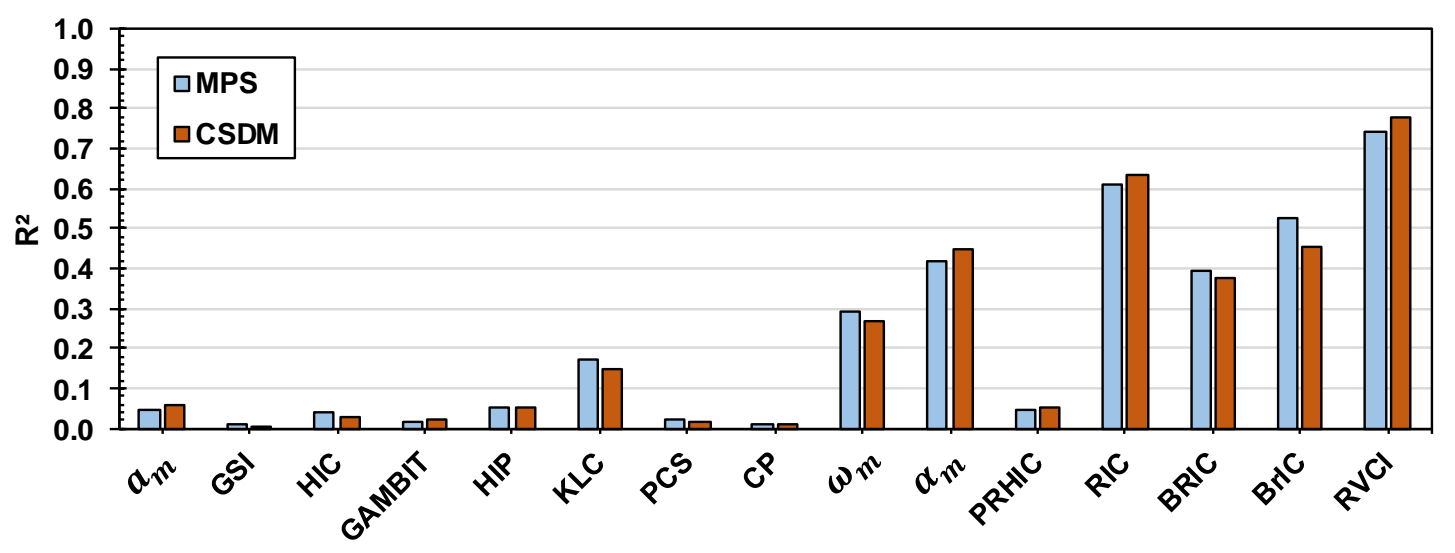

Figure 3-3: Correlations between kinematic and strain-based metrics for (A) frontal $(n=325)$, (B) oblique $(n=163)$, (C) side $(n=144)$, and (D) pedestrian $(n=$ 28) impact conditions. Results are based on GHBMC.

(A) MPS $\left(95^{\text {th }}\right.$ percentile $)$

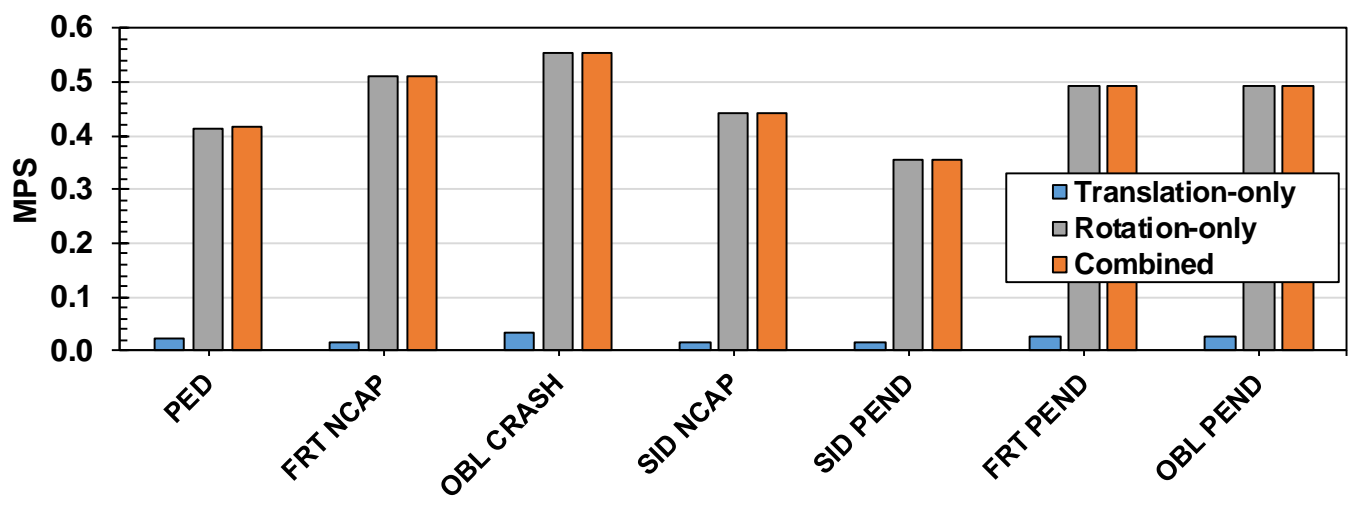

(B) $\operatorname{CSDM~(25\% ~MPS)~}$

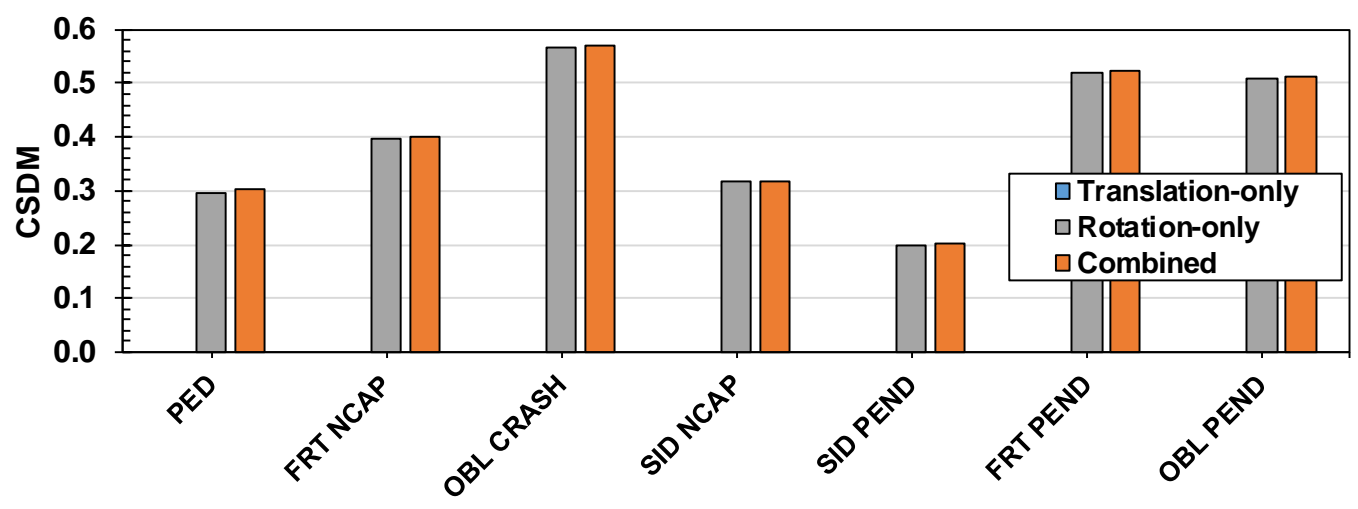

Figure 3-4: Brain strain sensitivity to translation-only, rotation-only, and combined head impact kinematics for seven cases with the highest severity HIC and resultant linear acceleration in the database; MPS $\left(95^{\text {th }}\right.$ percentile) (A), CSDM (25\% MPS) (B). Results are based on GHBMC. 
Table 3-3: Overall metric ranking by $R^{2}$ based on 660 head impacts.

\begin{tabular}{ccccc}
\hline \multirow{2}{*}{ Rank } & \multicolumn{2}{c}{ MPS } & \multicolumn{2}{c}{ CSDM } \\
\cline { 2 - 5 } & $($ GHBMC $)$ & (SIMon $)$ & $($ GHBMC $)$ & (SIMon) \\
\hline 1 & BrIC & RVCI & RVCI & BrIC \\
2 & RVCI & BrIC & BrIC & RVCI \\
3 & BRIC & $\omega_{m}$ & BRIC & $\omega_{m}$ \\
4 & RIC & RIC & RIC & RIC \\
5 & $\omega_{m}$ & $\alpha_{m}$ & $\alpha_{m}$ & BRIC \\
6 & $\alpha_{m}$ & BRIC & $\omega_{m}$ & $\alpha_{m}$ \\
7 & KLC & KLC & KLC & KLC \\
8 & PRHIC & PRHIC & PRHIC & PRHIC \\
9 & HIP & HIP & HIP & HIP \\
10 & GSI & GSI & GSI & GSI \\
11 & PCS & PCS & PCS & PCS \\
12 & CP & CP & CP & CP \\
13 & HIC & HIC & HIC & HIC \\
14 & GAMBIT & GAMBIT & GAMBIT & GAMBIT \\
15 & $a_{m}$ & $a_{m}$ & $a_{m}$ & $a_{m}$ \\
\hline
\end{tabular}

\subsection{Discussion}

The kinematic metric that establishes the severity of a head impact is some mathematical combination of the velocity and/or acceleration components of translational and/or rotational head motion. Although numerous metrics have been proposed for use in brain injury criteria, none have been universally accepted for a broad range of head impacts. In this chapter, the performance of fifteen kinematic-based metrics was assessed using FE model-predicted brain strains from simulations involving a broad range of head impacts taken from various head impact conditions. The relative performance of each kinematic metric was evaluated based on correlations with strain-based predictors of brain injury, MPS and CSDM from two different FE models.

Efficacy of MPS and CSDM as brain injury predictors is a vital assumption of this research. Several experimental studies have identified strain-based measures to be good predictors of diffuse brain injury and functional impairment using animal models (Bain 
and Meaney, 2000; MEANEY et al., 1995; Thibault et al., 1990). Although experimentally measured brain-tissue strains are preferred, they are difficult to obtain due to complexity of the brain and challenges associated with testing. Other studies have leveraged FE brain models to measure various mechanical variables including strain, strain rate, stress, and pressure for correlations with brain injury data from simulated impacts involving animal injuries or field studies involving humans (Bandak et al., 2001; Kleiven, 2007; Marjoux et al., 2008; Takhounts et al., 2008, 2013; Zhang et al., 2004). For example, Takhounts et al., 2008 and Kleiven 2007 found that metrics based on strain predicted diffuse brain injuries better when compared to other tissue-level responses (pressure). Although animal data offers potentially useful information, the assumptions associated with scaling brain deformations and injury tolerances to humans are problematic (Panzer et al., 2014). Furthermore, existing field impact databases are dominated by non-injury data, underreporting of concussion injuries, and rely on kinematics data from sensor systems that are not rigidly coupled to the head, e.g., (Rowson and Duma, 2013).

In this assessment, MPS was defined as the $95^{\text {th }}$ percentile (by element) maximum principal strain of all the elements in the brain rather than the $100^{\text {th }}$ percentile value as in previous studies (Kleiven, 2007; Takhounts et al., 2008, 2013). This was done to alleviate simulation results of the entire brain from being driven by the response of a single element, which may lead to erroneous results (Panzer et al., 2012). The overall correlation between many of the fifteen kinematic-based metrics and $95^{\text {th }}$ percentile MPS was higher in both FE models than with the $100^{\text {th }}$ response ( $\mathrm{R}^{2}$ values were nearly 0.1 higher); however, trends in the relative performance of kinematic metrics were not affected using the $100^{\text {th }}$ percentile MPS. Furthermore, the $95^{\text {th }}$ percentile MPS correlated better with CSDM $\left(\mathrm{R}^{2}=0.939\right.$ vs. 
0.806 for the $100^{\text {th }}$ percentile value). Based on these results, future work should focus on the $95^{\text {th }}$ percentile peak MPS response.

Table 3-4: Linear regression results using 660 head impacts and GHBMC.

\begin{tabular}{|c|c|c|c|c|c|c|}
\hline \multirow[b]{2}{*}{ Metric } & \multicolumn{3}{|c|}{ MPS } & \multicolumn{3}{|c|}{ CSDM } \\
\hline & $\begin{array}{c}\text { Intercept } \\
\left(\beta_{0}\right)\end{array}$ & $\begin{array}{l}\text { Slope } \\
\left(\beta_{1}\right)\end{array}$ & $\mathrm{R}^{2}$ & $\begin{array}{c}\text { Intercept } \\
\left(\beta_{0}\right)\end{array}$ & $\begin{array}{l}\text { Slope } \\
\left(\beta_{1}\right)\end{array}$ & $\mathrm{R}^{2}$ \\
\hline$a_{m}$ & $3.20 \mathrm{E}-1$ (8.33E-3) & $2.08 \mathrm{E}-4(6.18 \mathrm{E}-5)$ & 0.017 & $1.61 \mathrm{E}-1(1.14 \mathrm{E}-2)$ & $3.41 \mathrm{E}-4(8.43 \mathrm{E}-5)$ & 0.024 \\
\hline GSI & $2.66 \mathrm{E}-1(6.86 \mathrm{E}-3)$ & $1.06 \mathrm{E}-4(7.25 \mathrm{E}-6)$ & 0.246 & $8.69 \mathrm{E}-2$ (9.19E-3) & $1.55 \mathrm{E}-4(9.72 \mathrm{E}-6)$ & 0.279 \\
\hline $\mathrm{HIC}$ & $2.95 \mathrm{E}-1(6.99 \mathrm{E}-3)$ & $1.04 \mathrm{E}-4(1.11 \mathrm{E}-5)$ & 0.119 & $1.26 \mathrm{E}-1(9.43 \mathrm{E}-3)$ & $1.59 \mathrm{E}-4(1.50 \mathrm{E}-5)$ & 0.146 \\
\hline GAMBIT & $3.11 \mathrm{E}-1(8.49 \mathrm{E}-3)$ & $7.10 \mathrm{E}-2(1.53 \mathrm{E}-2)$ & 0.032 & 1.48E-1 (1.16E-2) & $1.11 \mathrm{E}-1(2.09 \mathrm{E}-2)$ & 0.041 \\
\hline HIP & $2.52 \mathrm{E}-1(6.84 \mathrm{E}-3)$ & $2.84 \mathrm{E}-6(1.67 \mathrm{E}-7)$ & 0.307 & $6.80 \mathrm{E}-2(9.19 \mathrm{E}-3)$ & 4.07E-6 (2.24E-7) & 0.334 \\
\hline KLC & $1.73 \mathrm{E}-1(8.98 \mathrm{E}-3)$ & $5.71 \mathrm{E}-1(2.71 \mathrm{E}-2)$ & 0.402 & $-4.58 \mathrm{E}-2(1.19 \mathrm{E}-2)$ & $8.21 \mathrm{E}-1(3.59 \mathrm{E}-2)$ & 0.443 \\
\hline PCS & $2.65 \mathrm{E}-1(7.99 \mathrm{E}-3)$ & $3.84 \mathrm{E}-3(3.23 \mathrm{E}-4)$ & 0.177 & $8.34 \mathrm{E}-2(1.07 \mathrm{E}-2)$ & $5.69 \mathrm{E}-3(4.34 \mathrm{E}-4)$ & 0.207 \\
\hline $\mathrm{CP}$ & $3.75 \mathrm{E}-1(5.74 \mathrm{E}-3)$ & $1.13 \mathrm{E}-2(1.09 \mathrm{E}-3)$ & 0.141 & $2.45 \mathrm{E}-1(7.76 \mathrm{E}-3)$ & $1.67 \mathrm{E}-2(1.47 \mathrm{E}-3)$ & 0.164 \\
\hline$\omega_{m}$ & 7.96E-2 (9.82E-3) & 7.02E-3 (2.46E-4) & 0.554 & $-1.75 \mathrm{E}-1(1.29 \mathrm{E}-2)$ & $9.95 \mathrm{E}-3(3.22 \mathrm{E}-4)$ & 0.593 \\
\hline$\alpha_{m}$ & $1.78 \mathrm{E}-1(6.78 \mathrm{E}-3)$ & $4.52 \mathrm{E}-2(1.60 \mathrm{E}-3)$ & 0.549 & $-3.73 \mathrm{E}-2(8.80 \mathrm{E}-3)$ & $6.44 \mathrm{E}-2(2.07 \mathrm{E}-3)$ & 0.595 \\
\hline PRHIC & $3.09 \mathrm{E}-1(4.65 \mathrm{E}-3)$ & $1.82 \mathrm{E}-8(1.01 \mathrm{E}-9)$ & 0.328 & $1.49 \mathrm{E}-1(6.18 \mathrm{E}-3)$ & 2.64E-8 (1.35E-9) & 0.367 \\
\hline RIC & $2.69 \mathrm{E}-1(4.22 \mathrm{E}-3)$ & $8.12 \mathrm{E}-9$ (2.76E-10) & 0.569 & $9.31 \mathrm{E}-2(5.51 \mathrm{E}-3)$ & $1.15 \mathrm{E}-8(3.60 \mathrm{E}-10)$ & 0.609 \\
\hline BRIC & 8.42E-2 (8.44E-3) & $3.17 \mathrm{E}-1(9.60 \mathrm{E}-3)$ & 0.623 & $-1.72 \mathrm{E}-1(1.06 \mathrm{E}-2)$ & $4.54 \mathrm{E}-1(1.21 \mathrm{E}-2)$ & 0.682 \\
\hline $\mathrm{BrIC}$ & $-3.02 \mathrm{E}-5(7.53 \mathrm{E}-3) \mathrm{a}$ & $4.62 \mathrm{E}-1(9.61 \mathrm{E}-3)$ & 0.778 & $-2.70 \mathrm{E}-1(1.04 \mathrm{E}-2)$ & $6.31 \mathrm{E}-1(1.33 \mathrm{E}-2)$ & 0.773 \\
\hline RVCI & $1.26 \mathrm{E}-1(6.08 \mathrm{E}-3)$ & 7.71E-3 (1.93E-4) & 0.709 & $-1.45 \mathrm{E}-1$ (7.47E-3) & $9.42 \mathrm{E}-3(1.86 \mathrm{E}-4)$ & 0.797 \\
\hline
\end{tabular}

Regression model parameter estimation (standard error).

${ }^{a}$ Indicates a non-significant result ( $\left.\mathrm{p}>0.05\right)$.

When predicting FE model brain strain, rotational-based kinematic metrics performed better than translational-based metrics. This result was not surprising given the amount of theoretical, experimental, and computational work available in the literature to support this finding. Holbourn theorized that the incompressible nature of brain tissue makes it susceptible to shear deformations during rotational motion of the head, and negligible deformations during translational motion (Holbourn, 1943). Experimentally, several studies have observed that rotational parameters head motion are more useful in predicting brain injury than translational parameters (Gennarelli et al., 1971, 1979; Ommaya and Gennarelli, 1974; Ommaya et al., 1964). Ommaya et al., 1974 observed no 
symptoms of cerebral concussion in specimens subjected to pure linear acceleration, while the opposite was true when only pure rotations were applied. Other studies, using FE brain models and have demonstrated low correlation between translational kinematic parameters and FE strains (Kleiven, 2007; Takhounts et al., 2008, 2013). For example, through development of BrIC, Takhounts et al., 2013 reported low correlation between translational kinematic metrics (linear acceleration and HIC), and MPS and CSDM from SIMon.

Compared to pure rotational motions, pure translational motions resulted in low strain despite having relatively high values for resultant linear acceleration and HIC. This finding is due to the near incompressibility of the brain tissue, where pure translational motions, which generate dilatational strains, contribute to negligible brain deformation. These result agrees with a previous study where a series of idealized pure linear accelerations were applied in the sagittal plane of the SIMon model. In that study, DAI risk was found to be less than 5\%, based on MPS and CSDM for pulses with magnitudes up to $120 \mathrm{~g}$ and durations between (1-100) ms (Gabler et al., 2016). Furthermore, when simulating only the rotational components, strain-based responses were nearly identical to the combined 6DOF head motion. This result suggests that brain strain responses can be adequately represented using only the rotational components of a head impact.

Metrics based on angular velocity had the highest correlation with MPS and CSDM (Figure 3-1). For each impact condition either BrIC or RVCI was the top performing metric. This result was not surprising given that both of these metrics were developed from FE model responses. A majority of the head impacts used in the current study were also used by Takhounts et al., 2013 to develop BrIC, while RVCI was developed using 
correlations with MPS and CSDM based on head impacts acquired from frontal and pedestrian whole-body, dummy crash test simulations.

However, both BrIC and RVCI have limitations: For pedestrian impact events, which had head angular velocities longer in duration than the other three crash modes, BrIC was not well correlated $\left(\mathrm{R}^{2} \sim 0.5\right)$ for MPS and CSDM. One way to improve correlations between BrIC and FE strains in longer duration events, would be to include angular acceleration in the formulation. This was accomplished by Takhounts et al., 2011 in an earlier version of the criterion (BRIC); however, Takhounts et al., 2013 found low correlation between angular acceleration and FE strains, and thus excluded it from the mathematical form. In the current study, correlations between angular acceleration and strain-based metrics were good overall, and higher than correlations for angular velocity in side and pedestrian impact conditions. This may be due to inclusion of longer duration impacts in these conditions including pedestrian and far side, which were not investigated by Takhounts et al., 2013. For this dissertation, angular acceleration was obtained by differentiating filtered angular velocity data, since protocols for processing angular acceleration data from various dummies and impact conditions have not been established.

RVCI was better correlated to MPS and CSDM for pedestrian impacts than BrIC, and the integration limits selected for RVCI appear to be well tuned to the longer duration event. However, for shorter duration head impacts, mainly those generated from pendulum impact, RVCI did not correlate as well as BrIC (and thus, BrIC was overall a more predictive metric). Although both BrIC and RVCI have been shown to correlate well with FE model strains over a broad range of head impact conditions, further work is necessary 
to develop a more robust kinematic metric, which can accurately predict tissue-level FE strains over all durations.

When comparing the results of this study with findings from similar studies, we observed mixed trends. Hernandez et al., 2015 evaluated several kinematic and FE-based brain injury metrics relative to injury outcomes using head impacts from collegiate football, boxing, and mixed martial arts (Hernandez et al., 2015). Based on their assessment, maximum resultant angular acceleration, GAMBIT, and HIP were among the best kinematic-based predictors of the injury data, while BrIC and maximum resultant angular velocity performed similar to translation-only metrics. This preliminary investigation involved 110 head impacts of which only two injuries were used in the assessment, so their conclusions may potentially change with additional injury data. Brain injury metrics have also been evaluated using field data from automotive-related crashes to assess the efficacy of kinematic-based brain injury criteria, e.g., (Hasija et al., 2007; Laituri et al., 2015, 2016; Mueller et al., 2015; Prasad et al., 2014). Results from these studies found that injury rates predicted by HIC and CP were more closely matched with real-world rates than BrIC. However, these field studies do not specifically assess the kinematic metric of $\mathrm{HIC}, \mathrm{CP}$, or BrIC, they assess the injury risk functions associated with these metrics, which is an issue not addressed in this dissertation.

Previously, Ji et al., 2014 evaluated three independently developed and validated FE models of the human head by checking consistency across several brain mechanical responses ( $\mathrm{Ji}$ et al., 2014). They observed remarkable differences in every tissue-level response variable assessed. In this study, two different FE models of the human head (SIMon and GHBMC) were used, and strain results were compared to assess consistency. 
The correlation trends were similar regardless of the FE model, with rotational-based metrics performing better than translational ones. Specifically, BrIC and RVCI were the best performing metrics and maximum resultant linear acceleration had the lowest correlation with MPS and CSDM. Consistency between the models suggests that the results of this assessment are not model dependent.

\subsection{Conclusions}

The performance of fifteen kinematic-based brain injury metrics was assessed by comparing their correlations with tissue-level strain responses obtained from 660 head impacts simulated in two different FE human head models. Results revealed rotation-onlybased metrics to have higher correlation with FE model strains when compared to combined rotation-translation and translation-only metrics. Based on these findings, and the FE models used to derive strain-based metrics, kinematic brain injury criteria targeting clos injuries, including DAI and concussion, should be based solely on rotational head kinematics. Furthermore, BrIC and RVCI, were found to have the highest overall correlation with this dataset. Although these metrics showed high capability for predicting FE model strains, their efficacy was found to be limited to a specific regime of loading. Future development of kinematic-based brain injury criteria should look to improve upon the formulation of these two metrics. 


\section{Chapter 4 - Brain Deformation Physics}

Establishing a link between head kinematics and injury risk has been the focus of numerous brain injury criteria. While many early metrics were developed using mechanics principles, recent criteria have typically been developed using empirical methods often based on subsets of head impact data. In this chapter, the relationship between rotational head motion and brain deformation is explored using a second-order mechanical system analog. This chapter was published previously (Gabler et al., 2017. Development of a Single-Degree-of-Freedom Mechanical Model for Predicting Strain-based Brain Injury Responses. Journal of Biomechanical Engineering; DOI:10.1115/1.4038357) and was adapted for this dissertation with permission from the publisher.

\subsection{Introduction}

Early development of head and brain injury criteria was largely based on physics, where fundamental mechanics principles were used to develop models to describe human tolerance to head impact, e.g., (Brinn and Staffeld, 1970; Fan, 1971; Slattenschek and Tauffkirchen, 1970; Stalnaker et al., 1971). These models consisted of spring-mass-damper systems that were constructed to represent the motion of the skull and brain under head impact (Figure 1-6). Impacts were typically simulated by applying kinematic pulses to the base of the model (skull), while the resulting deformation of the compliant elements and motion of the mass (brain) was calculated. The relative motion between the mass and base was believed to be related to various forms of injury including skull fracture, subdural hematoma, concussion, and DAI (Brinn and Staffeld, 1970; Fan, 1971; Goldsmith, 1972; Holbourn, 1943; Slattenschek and Tauffkirchen, 1970; Stalnaker et al., 1971). 
Despite the plausibility of a physics-based approach, many existing brain injury criteria were developed using empirical methods, e.g., Gadd's severity index, which was developed as an approximation to the WSTC (Gadd, 1966), and then later HIC (Versace, 1971). While empirically derived models are preferred for their ability to quickly describe trends in the data, they are often developed from fits that only correlate well with limited datasets. However, these correlations may not hold outside the experimental data, and an empirical-based injury criterion may lead to nonsensical predictions when extrapolated beyond the range of data used to fit the model. Ideally, a brain injury criterion should be derived from fundamental mechanics principles first, and then verified with experimental data, rather than being developed from subsets of data using empirical methods.

In Chapter 3, rotational kinematics were found to be the best correlates to FE-based brain injury metrics MPS and CSDM using a large dataset head impacts. Although FE models are considered state-of-the-art brain injury assessment tools, given their level of sophistication and computational cost, they are often difficult to summarize for arbitrary head impacts, and impractical to use for rapid risk assessment in regulatory testing and onfield evaluations using wearable sensors. Thus, it is necessary to have a tool that can predict tissue-level strain response with the accuracy of an FE model, while maintaining the simplicity of a kinematic-based metric (Figure 1-7). Given that strain is believed to be a primary mechanism for brain injury, an effective kinematic-based criterion should be formulated using only parameters that are relevant to the development of brain strain.

In this chapter, a single-degree-of-freedom (sDOF) mechanical system was used to develop a theoretical framework for understanding the relationship between rotational head kinematics and brain deformation. Efficacy of the sDOF model for predicting brain injury 
response was evaluated by comparing the deformation of the second order system to strainbased brain injury metrics obtained from the FE models. A parametric study was performed where the magnitude and duration of an idealized angular acceleration pulse were varied over a broad range of values and applied to the sDOF model, and separately about each axis of the FE model to develop a fundamental understanding of brain deformation mechanics under rotational head motion. Performance of the sDOF model was then assessed relative to existing rotational-based metrics using the head impact data from Chapter 3. This chapter identifies key kinematic parameters and their relationship to brain deformation, which is essential to the development of an improved brain injury criterion.

\subsection{Methods}

\subsubsection{Single-Degree-of-Freedom Mechanical Model}

A damped, sDOF mechanical system with base excitation was developed to study the relationship between rotational head motion and brain deformation (Figure 4-1). The equation of motion (EOM) for this system is given by the following expression:

$m \ddot{w}+c \dot{w}+k w=c \dot{u}+k u$

Equation 4-1

where $\ddot{u}, \dot{u}, u$ and $\ddot{w}, \dot{w}, w$ are the accelerations, velocities, and displacements of the base and mass, $m$, and $c$ and $k$ are the system parameters for the damping and stiffness, respectively. The relative base-mass displacement, $\delta=u-w$, was assumed to be analogous to brain deformation under rotational head motion. Substituting parameters for the system natural period, $2 \pi \sqrt{m k^{-1}}$, and damping ratio, $\zeta=c / \sqrt{4 m k}$, Equation 4-1 can be rewritten in the following form:

$\ddot{\delta}+4 \pi \zeta \Delta t_{n}^{-1} \dot{\delta}+4 \pi^{2} \Delta t_{n}^{-2} \delta=\ddot{u}$ 
In this dissertation, the sDOF model was assumed to be underdamped $(0<\zeta<1)$ and the solution to Equation 4-2, $\delta=\delta(t)$, where $t$ is time, was derived analytically using the particular solution given by Equation 4-4 in section 4.2.2. The maximum magnitude of displacement, $\delta_{m}$, was assumed to be analogous to maximum brain deformation:

$\delta_{m}=\max _{t}\{|\delta(t)|\}$

Equation 4-3

Further detail on the analytical solution to Equation 4-2 is provided in Appendix F.

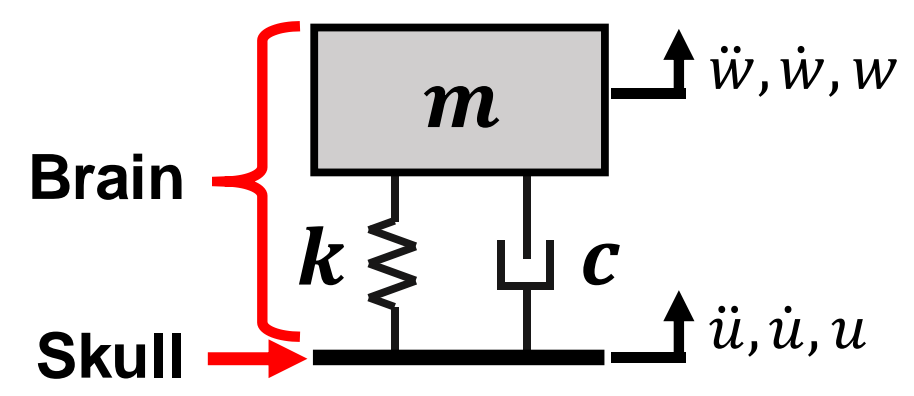

Figure 4-1: sDOF model with base excitation used as an analog for maximum brain deformation under rotational head motion.

\subsubsection{Head Kinematics}

A series of idealized rotational pulses were applied to the sDOF and FE models to study the relationship between head motion and maximum brain deformation. Translational head kinematics were not investigated in this chapter, since they were shown to have poor correlation with brain strain in Chapter 3. A sinusoidal pulse shape was chosen for the parametric study, because it has been used extensively in impact biomechanics research, e.g., (Gierke, 1964; Takhounts et al., 2013) and allows for an analytical approach to solving the EOM of the sDOF model. Specifically, a single cycle in angular acceleration was used with magnitude, $\ddot{u}_{m}$, and duration, $\Delta t$ (Figure 4-2):

$$
\ddot{u}(t)=\ddot{u}_{m}\left\{\begin{array}{cc}
\sin \left(\frac{2 \pi t}{\Delta t}\right) & 0 \leq t<\Delta t \\
0 & \Delta t \leq t<t_{\text {ext }}
\end{array}\right\} .
$$


Integrating Equation 4-4 we obtain the expression for the angular velocity time history:

$\dot{u}(t)=\frac{\dot{u}_{m}}{2}\left\{\left[\begin{array}{cc}\left.1-\cos \left(\frac{2 \pi t}{\Delta t}\right)\right] & 0 \leq t<\Delta t \\ 0 & \Delta t \leq t<t_{\text {ext }}\end{array}\right\}\right.$,

Equation 4-5

where $\dot{u}_{m}$ is the magnitude of the angular velocity pulse. The expression that relates input pulse parameters (acceleration, velocity, and duration) for a sinusoidal pulse shape is

$\Delta t=\pi \frac{\dot{u}_{m}}{\ddot{u}_{m}}$.

Equation 4-6

(A)

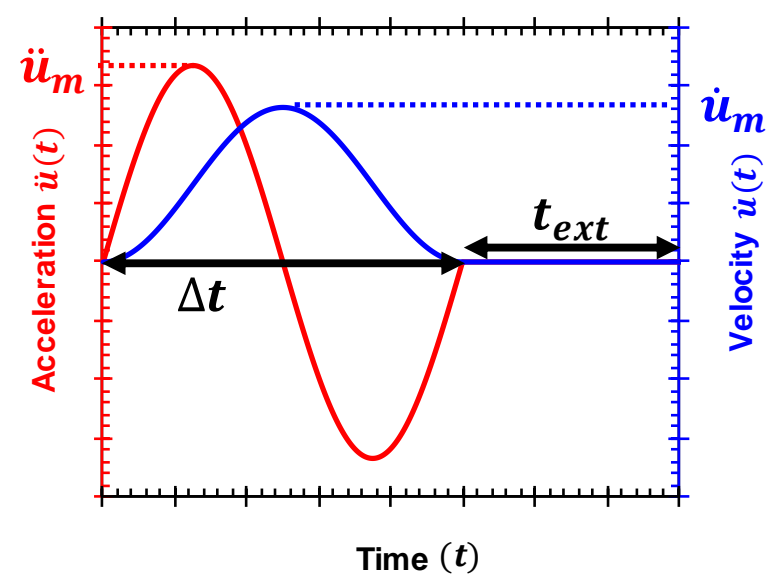

(B)

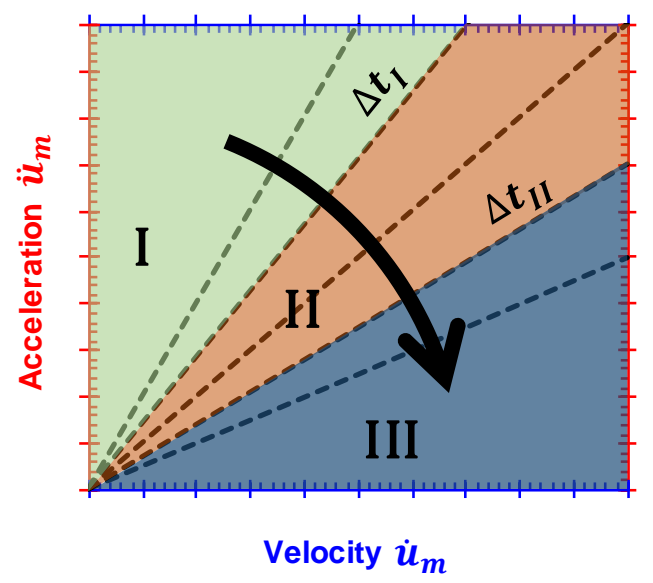

Figure 4-2: Idealized kinematic pulse applied to the FE and SDOF models (A) and the relationship between pulse parameters $\left(\ddot{u}_{m}, \dot{u}_{m}, \Delta t\right)$ in the design space (B). Increasing lines of constant pulse duration are indicated by the black arrow and dotted lines. Pulse duration is inversely related to the slope of the black dotted lines $(\pi / \Delta t)$ where $\Delta t_{I}<\Delta t_{I I}$. Short-duration pulses are indicated by Region I: $\Delta t<\Delta t_{I}$, moderate duration pulses are indicated by region II: $\Delta t_{I}<\Delta t<\Delta t_{I I}$ (system natural period is located in this region), and long-duration pulses are indicated by region III: $\Delta t_{I I}<\Delta t$.

Equation 4-6 indicates that only two of the three kinematic parameters $\left(\ddot{u}_{m}, \dot{u}_{m}\right.$, $\Delta t)$ are needed to define the pulse. Since real-world impacts often result in complex head kinematics, it can be difficult to define an impact duration. Therefore, $\ddot{u}_{m}$ and $\dot{u}_{m}$ were chosen as design variables for the parametric study (Figure 4-2), and focus was placed on 
establishing a relationship between the magnitudes of angular velocity and angular acceleration, and deformation-based metrics ( $\delta_{m}$, MPS, and CSDM).

Different pulses were constructed by varying the magnitudes of angular velocity and angular acceleration over a broad range of values including those typically experienced in automotive and football-related head impact environments. Specifically, $\ddot{u}_{m}$ and $\dot{u}_{m}$ were varied from $(0.1-15) \mathrm{krad} / \mathrm{s}^{2}$ and $(1-100) \mathrm{rad} / \mathrm{s}$, respectively, with 17 unique values for each variable (Figure 4-3). The range and distribution of kinematic parameters chosen were based on the 660 head impacts from sled, crash, and pendulum tests (Chapter 3). Simulations were selected at a higher frequency for lower values of $\ddot{u}_{m}$ and $\dot{u}_{m}$ due to the abundance of real-world head impact data located there (Figure 4-3).

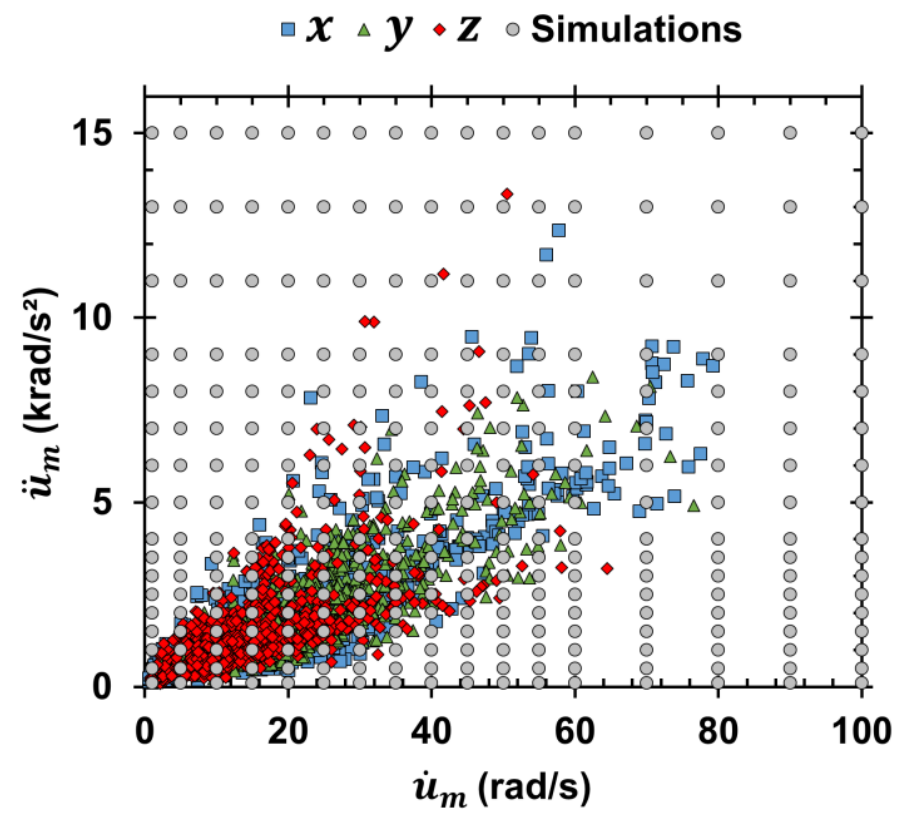

Figure 4-3: Design space for numerical simulations used in the parametric study. Idealized rotational pulses applied to the SDOF and FE models were constructed using Equations 4-4 through 4-6 for the gray, circular grid points shown above (Simulations). Colored data points are the directionally dependent $(x, y, z)$ values for $\ddot{u}_{m}$ and $\dot{u}_{m}$, which were taken from the 660 sled, crash, and pendulum tests (Chapter 3), and used to define the range and distribution of pulses for the parametric study (Simulations). 
For each value of $\ddot{u}_{m}$ and $\dot{u}_{m}$, the corresponding pulse duration, $\Delta t$, was calculated using Equation 4-6, which enabled calculation of the angular acceleration and angular velocity time histories; Equations 4-4 and 4-5. Pulse durations spanned roughly ( $1-3000)$ ms. An additional $100 \mathrm{~ms}$ of time, $t_{\text {ext }}$, was added to each pulse to ensure that all deformation-based metrics had achieved their maximum value following the input motion.

\subsubsection{Numerical Simulations}

Angular velocity time histories were applied to the rigid skull of both FE models, separately, about each of the three local head axes. Rotations were performed about the positive $x$ and $z$ axes, and negative $y$ axis. Due to symmetry of the brain model, rotations could be applied about the $x$ and $z$ axes in either the positive or negative directions. For rotations around the $y$ axis, symmetry does not hold, and additional simulations were performed about the positive $y$ axis to check consistency of MPS and CSDM between the

different directions. A total of 916 simulations were performed in GHBMC; 289 simulations about each anatomical axis $(+x,+z$, and $-y)$ and 49 simulations about the $+y$ axis. A subset of these simulations (130 per direction; $(+x,+z$, and $-y$, and 49 simulations about the $+y$ axis) were performed in SIMon to check consistency with GHBMC. MPS and CSDM were calculated for each simulation, and then related back to the input kinematics ( $\ddot{u}_{m}$ and $\dot{u}_{m}$ ) to form a deformation response surface (contour plot).

The relationship between applied kinematics and maximum deformation in the sDOF model was rigorously studied by solving Equation 4-2 for a broad range of kinematic inputs $\left(\ddot{u}_{m}\right.$ and $\left.\dot{u}_{m}\right)$ and system parameters $\left(\Delta t_{n}\right.$ and $\left.\zeta\right)$. Following this preliminary investigation, the sDOF model was fit to a subset of the uniaxial MPS responses from 
GHBMC to make a more comprehensive assessment of the model's capability to predict brain strain. Fitting was performed by assuming $\delta_{m}$ to be correlated with MPS:

$M P S=\beta \delta_{m}$

Equation 4-7

Values for the system parameters $\left(\Delta t_{n}\right.$ and $\left.\zeta\right)$ and regressor $\beta$ were determined using a nonlinear, least-squares solver (lsqcurvefit; Matlab, v8.4.0, The MathWorks, Natick, Ma) to minimize the sum squared error (SSE) between GHBMC-measured and sDOF-predicted MPS. An optimized set of coefficients were determined independently for each anatomical direction of the FE model. All simulations for the sDOF model were performed in Matlab (v8.4.0, The MathWorks, Natick, Ma) on a standard desktop computer (Intel Core i5$5300 \mathrm{U}, 2.3 \mathrm{GHz}, 8 \mathrm{~GB})$.

The subset of uniaxial GHBMC MPS responses used for fitting the parameters of the sDOF model included parametric cases with kinematic characteristics $\left(\ddot{u}_{m}, \dot{u}_{m}, \Delta t\right)$ similar to those from the 660 the sled, crash, and pendulum tests. To accomplish this, pulse magnitudes and effective durations were calculated for each of the 660 arbitrary impacts: Directionally dependent magnitudes $\left(\ddot{u}_{m}, \dot{u}_{m}\right)$ were obtained by taking the maximum magnitude of the angular velocity and angular acceleration time history signals in each direction. These values were then used in Equation 4-6 to obtain an effective pulse duration (based on a sinusoid approximation) for each axis of the head. This resulted in three distributions ( $n=660$, one for each axis of the head) of maximum pulse magnitudes and effective durations for the real-world cases. The overall maximum angular velocity and angular acceleration magnitudes $\left(\ddot{u}_{m}, \dot{u}_{m}\right)$ along with the maximum and minimum effective durations $\left(\Delta t_{\min }, \Delta t_{\max }\right)$ were selected from each distribution/direction, and used to define boundaries from which to select uniaxial cases for sDOF model fits (Table 4-1). 
Using these subsets of the parametric data reduced potential issues with fitting sDOF model responses to higher severity, and very short or very long duration outcomes, for which the FE models have not been validated. Only parametric cases with durations between (6157) ms were used for fitting. Shorter and longer duration cases were used for qualitatively comparing sDOF and FE model deformation response patterns.

Table 4-1: Range of parametric simulations used for SDOF model fits.

\begin{tabular}{ccccc}
\hline Direction & $\begin{array}{c}\ddot{u}_{m} \\
\left(\mathrm{krad} / \mathrm{s}^{2}\right)\end{array}$ & $\begin{array}{c}\dot{u}_{m} \\
(\mathrm{rad} / \mathrm{s})\end{array}$ & $\begin{array}{c}\Delta t_{\min } \\
(\mathrm{ms})\end{array}$ & $\begin{array}{c}\Delta t_{\max } \\
(\mathrm{ms})\end{array}$ \\
\hline$x$ & $0-13$ & $0-80$ & 6 & 142 \\
$y$ & $0-8$ & $0-80$ & 10 & 135 \\
$z$ & $0-13$ & $0-60$ & 7 & 157 \\
\hline
\end{tabular}

\subsection{4 sDOF Model Performance and Comparison with Existing Metrics}

The sDOF model was assessed for application in real-world head impact conditions using the head impacts from Chapter 3. Angular acceleration time histories for each test were applied separately to their respective directionally dependent, fitted sDOF model. Model displacement time histories were calculated numerically (ode45; Matlab, v8.4.0, The MathWorks, Natick, Ma) and their maximum magnitudes were used in Equation 4-7 to obtain a MPS prediction for each anatomical direction. Directionally dependent MPS predictions were then root sum squared to obtain the total predicted MPS. Numerical solutions to Equation 4-2 took no longer than 3 s to compute on a standard desktop computer. Correlations between the sDOF-predicted MPS and FE-measured MPS for the 660 head impacts used in Chapter 3 were compared to those for existing rotational brain injury metrics. Kinematic-to-strain correlations were also evaluated for each of the existing rotational metrics using the uniaxial data for each anatomical direction $(x, y$, and $z)$. 


\subsection{Results}

\subsection{1 sDOF Model Results}

The maximum magnitude of deformation from the sDOF model exhibited a nonlinear dependence on the magnitudes of angular velocity and angular acceleration. For different combinations of system parameters $\left(\Delta t_{n}\right.$ and $\left.\zeta\right)$, maximum brain deformation magnitude was governed by three general categories of rotational motion which were distinguished by the pulse duration relative to the system natural period: For short-duration pulses, maximum brain deformation magnitudes were represented as vertical contour lines on the acceleration-velocity plot (Figure 4-4), which indicated that brain deformation in this region was primarily a correlate of the magnitude of angular velocity. For longduration pulses, brain deformation magnitudes were represented as horizontal contour lines, which indicated that brain deformation in this region was primarily a correlate of the magnitude of angular acceleration (Figure 4-4). Between the short- and long-duration regions, brain deformation contour lines "turned the corner" and transitioned from a velocity-dependent response to an acceleration-dependent response. In this moderate duration region (which was represented by pulses with durations near the system natural period), maximum brain deformation magnitudes were dependent on a combination of angular velocity and angular acceleration magnitudes (Figure 4-4).

Based on the deformation patterns in Figure 4-4, the relative sizes of these three regions (short, long, and moderate duration) were found to be system dependent; given the same natural period, and velocity and acceleration magnitudes, non-normalized sDOF displacements were higher near system resonance as damping decreased. 

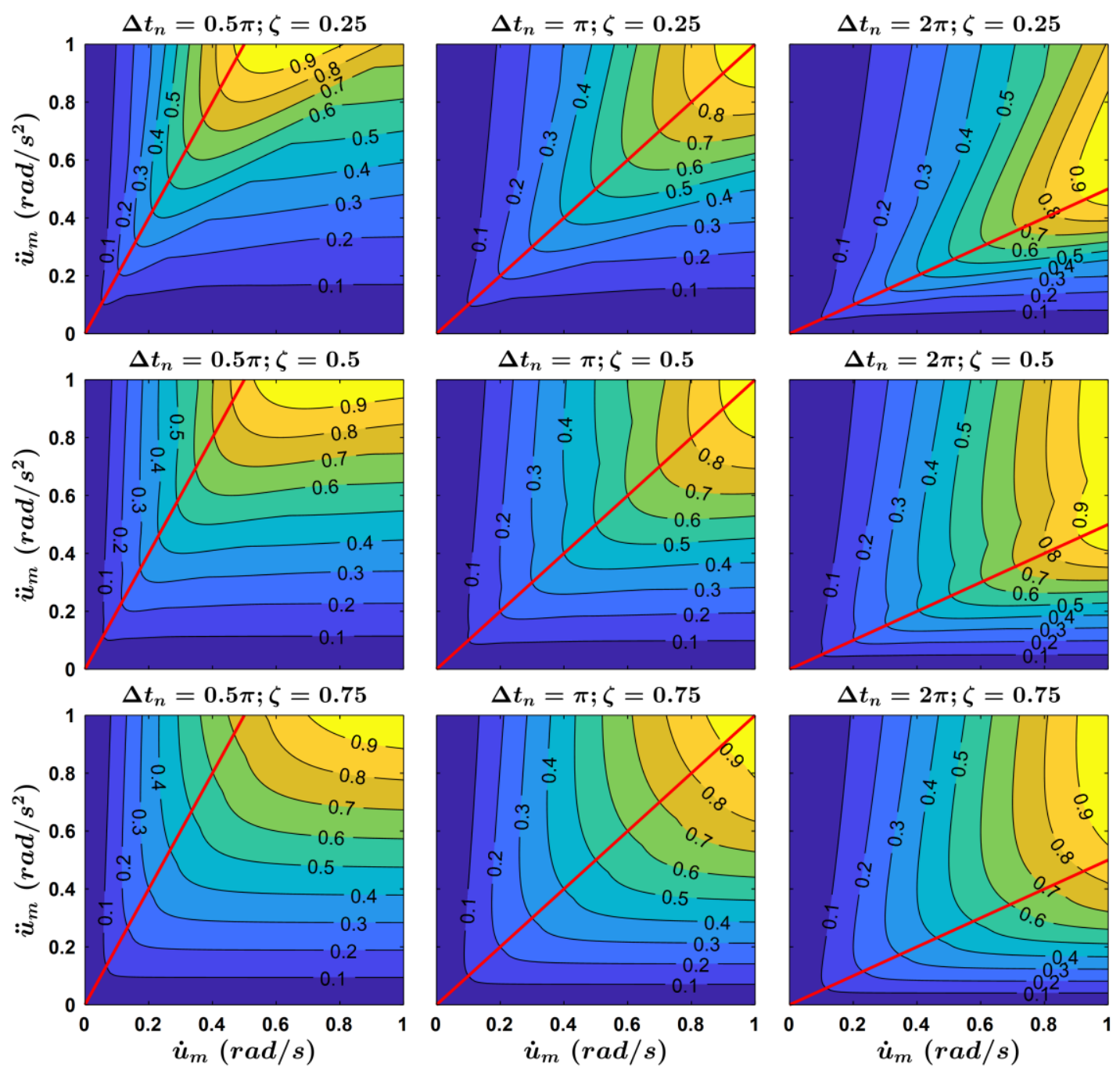

Figure 4-4: Contour plots showing the relationship between $\ddot{\boldsymbol{u}}_{m}, \dot{\boldsymbol{u}}_{m}$, and $\delta_{m}$ for various combinations of system parameters $\left(\Delta t_{n}\right.$ and $\left.\zeta\right)$ using the sDOF model.

Contour lines represent constant levels of $\delta_{m}$ which were normalized by the maximum value within the surface. The slope of the solid (red) line is inversely related to the system natural period $\left(\pi / \Delta t_{n}\right)$.

\subsubsection{FE Model Results}

Strain-based responses (MPS and CSDM) obtained from FE simulations using the idealized rotational pulses shared remarkable similarities to the response surfaces generated by the sDOF model. For short-duration pulses, the contours of MPS and CSDM were relatively vertical indicating a dependence primarily on the magnitude of angular 
velocity, while for long-duration pulses the contours were relatively horizontal indicating that strain responses were primarily dependent on the magnitude of angular acceleration (Figure 4-5). For moderate duration pulses, MPS and CSDM depended on a combination of angular velocity and angular acceleration, suggesting a resonance behavior of the brain. Most of the sled, crash, and pendulum head impacts were concentrated in the resonance region where strain-based responses were amplified and dependent on both velocity and acceleration (Figure 4-5).

Despite some differences, strain-based responses had similar characteristics between the two FE models. MPS and CSDM correlated well between GHBMC and SIMon; $\left(\mathrm{R}^{2}>0.9\right)$ for each metric, and in each anatomical direction. However, responses from SIMon were more erratic for moderate-to-longer duration pulses and exhibited a greater degree of dependence on angular velocity relative to those from GHBMC, which were smoother and more akin to the sDOF model (Figure 4-5). Responses became more nonlinear at higher severity kinematic inputs $\left(\dot{u}_{m}>60 \mathrm{rad} / \mathrm{s}\right.$ and $\left.\ddot{u}_{m}>7 \mathrm{krad} / \mathrm{s}^{2}\right)$; however, little of the sled, crash, and pendulum data were found above these levels. In both FE models, strains in the $y$ and $z$ directions were, in general, higher than those for the $x$ direction given similar kinematic inputs $\left(\ddot{u}_{m}\right.$ and $\left.\dot{u}_{m}\right)$. Also, both models had good agreement between strain-based responses generated in positive and negative $y$ direction rotations $\left(\mathrm{R}^{2} \approx 1\right)$, suggesting that MPS and CSDM values are independent of the direction of pure sagittal plane rotations (Figure 4-6). 

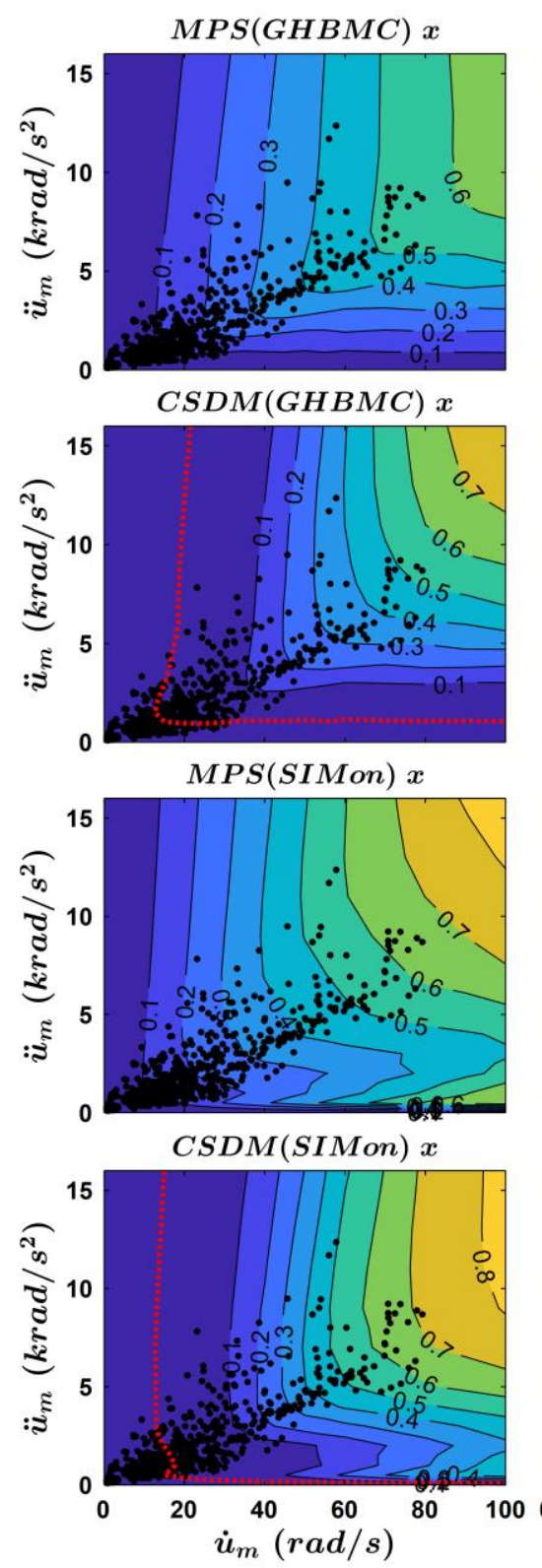
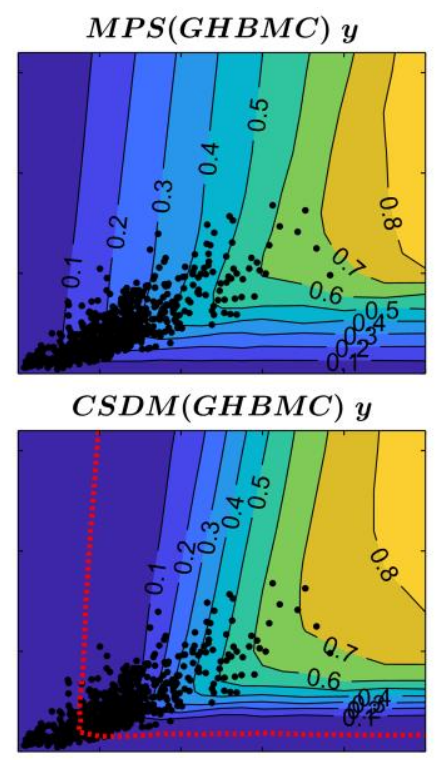

MPS(SIMon) $y$

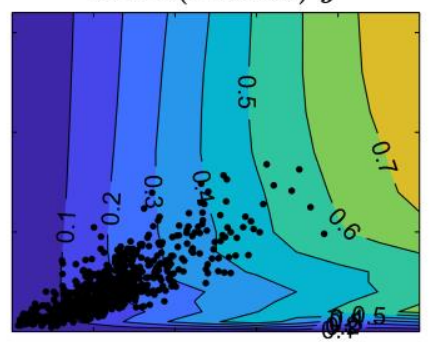

CSDM(SIMon) $y$

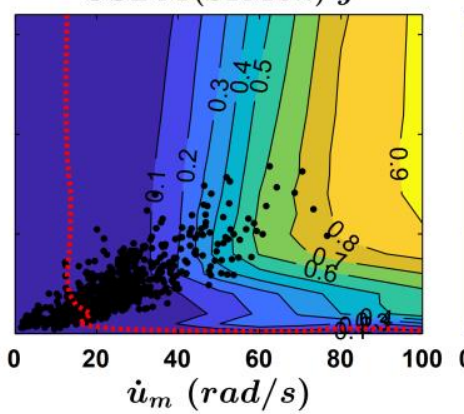

$\operatorname{MPS}(G H B M C) z$

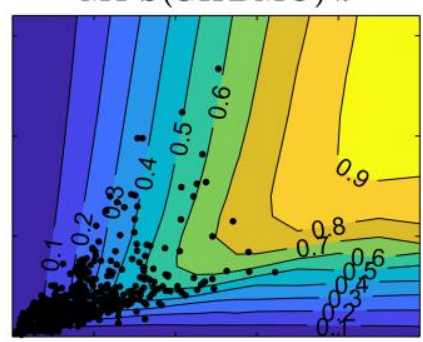

$C S D M(G H B M C) z$

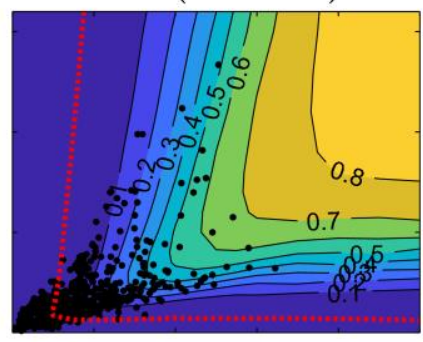

MPS(SIMon) $z$

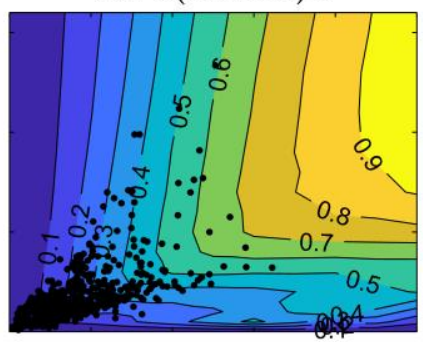

CSDM(SIMon) $z$

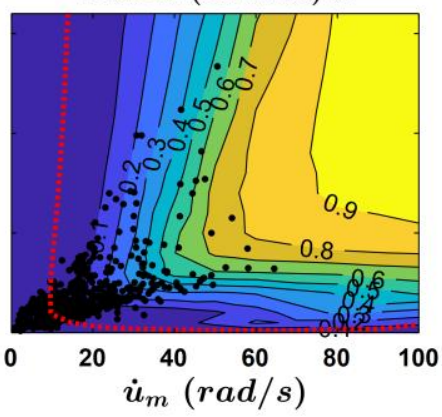

Figure 4-5: Contour plots showing the relationship between $\ddot{u}_{m}, \dot{u}_{m}$, and strainbased metrics obtained from numerical simulations using GHBMC and SIMon. Contour lines represent constant levels of MPS/CSDM. Directionally dependent $(x, y, z)$ values for $\ddot{u}_{m}$ and $\dot{u}_{m}$ were obtained from the sled, crash, and pendulum data, and are plotted (black dots) to show the location of real-world head impacts relative to the strain contours generated using the idealized pulses. Dotted lines (red) shown on CSDM plots indicate a maximum principal strain value of $25 \%$ $\left(100^{\text {th }}\right.$ percentile element $)$, which was used as the threshold for the onset of CSDM. 
(A)

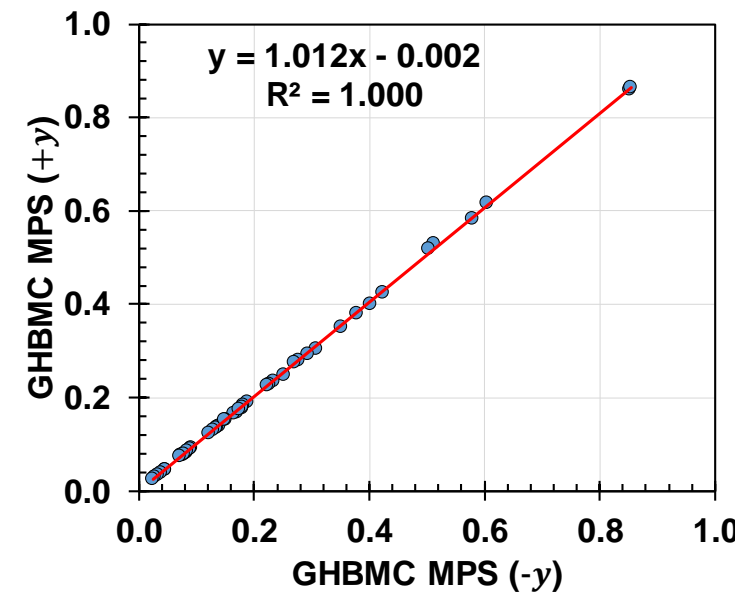

(C)

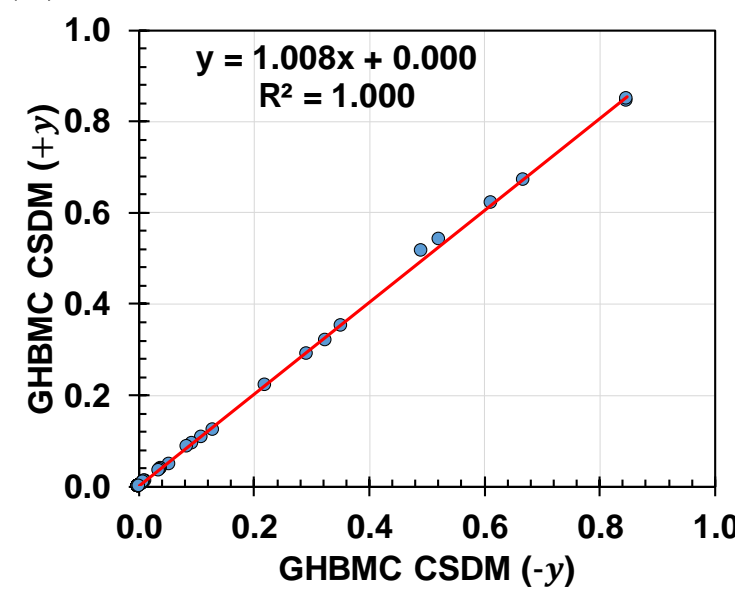

(B)

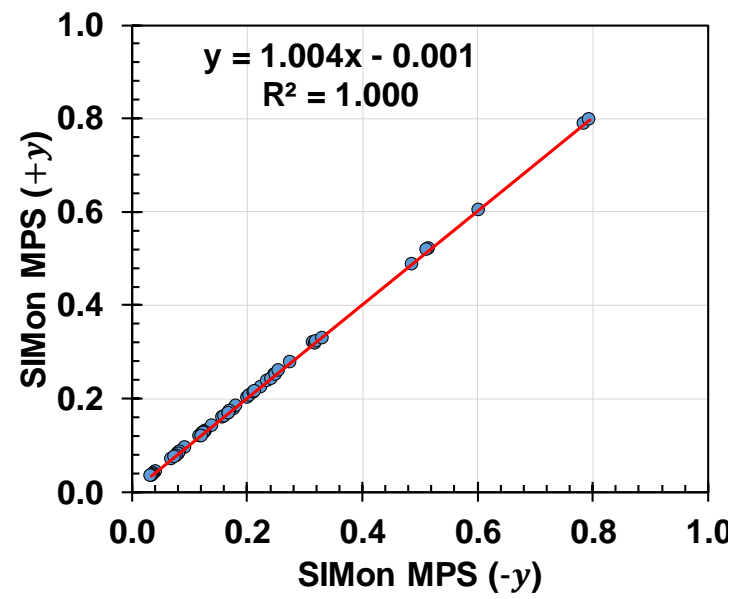

(D)

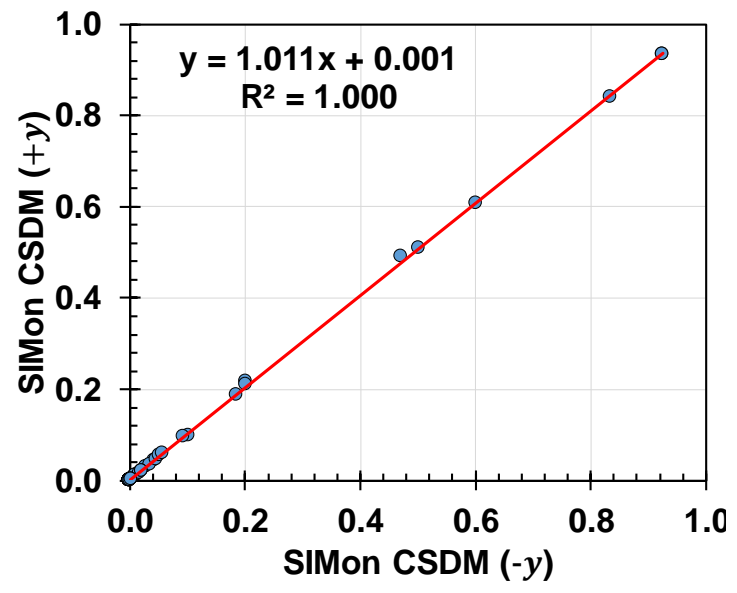

Figure 4-6: Sensitivity of MPS (A, B) and CSDM (C, D) from GHBMC (A, C) and SIMon (B, D) to rotational motions about $\pm y$ axis. Results are based on MPS $\left(9^{\text {th }}\right.$ percentile) and CSDM (25\% MPS).

\subsection{3 sDOF Model Parameter Identification.}

The sDOF model fit the subset of uniaxial MPS responses from GHBMC well; $\mathrm{R}^{2}$ $>0.980$ for each anatomical direction (Figure 4-7, Table 4-2). The natural period of the fitted SDOF model showed good agreement with the assumed location of the natural period from the MPS contours (Figure 4-8). Fits using the sDOF model yielded effective rotational brain natural periods between 36 and $45 \mathrm{~ms}$, while values for the system natural frequency, $\omega_{n}=2 \pi / \Delta t_{n}$, were between 140 and $173 \mathrm{rad} / \mathrm{s}$, depending on the anatomical direction. 
Fitted values and goodness of fit metrics for the directionally dependent system parameters $\left(\Delta t_{n}\right.$ and $\left.\zeta\right)$ and regression model coefficient $\beta$ are included in Table 4-2.

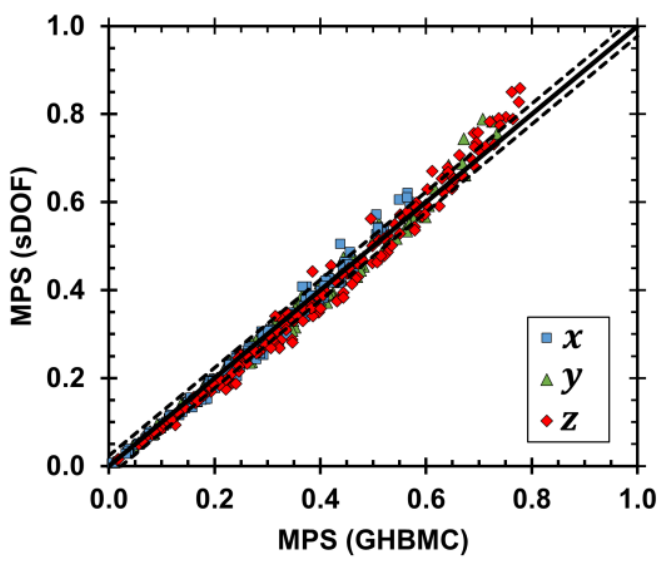

Figure 4-7: sDOF model responses fit to the subset of GHBMC simulations for each anatomical direction. The solid and dotted lines (black) indicate unity and \pm 1 root mean square error, respectively. Fitted values for the uniaxial parameters of the directionally dependent SDOF models are listed in Table 4-2.

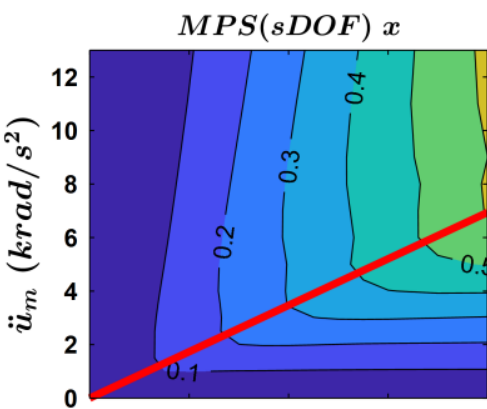

$\operatorname{MPS}(G H B M C) x$

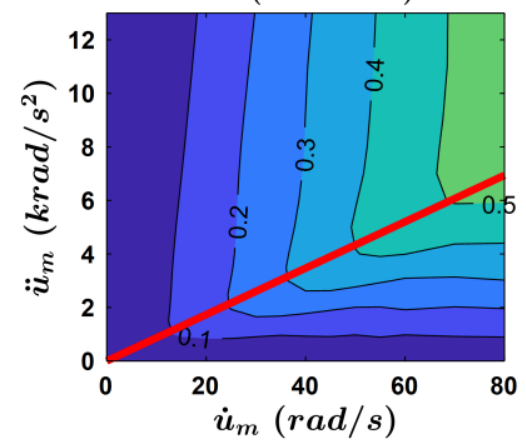

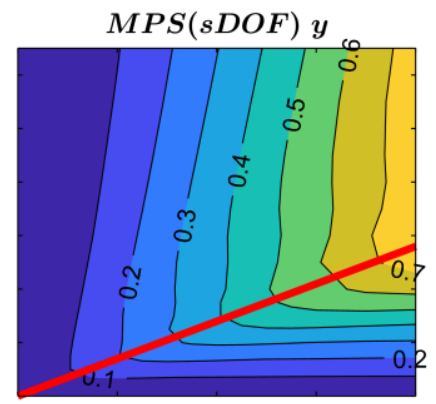

$\operatorname{MPS}(G H B M C) y$

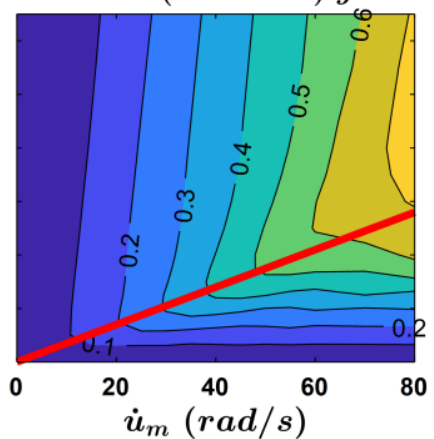

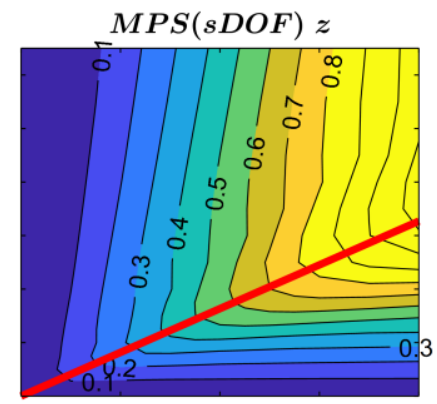

$\operatorname{MPS}(G H B M C) z$

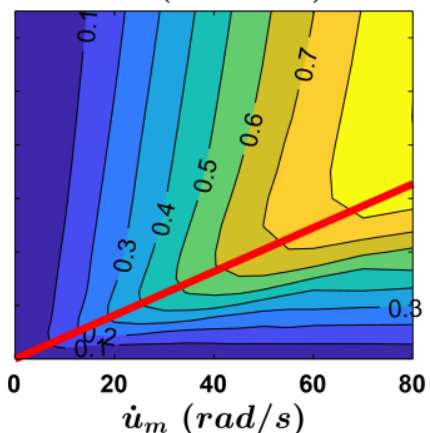

Figure 4-8: Contour plots showing a comparison between the fitted sDOF model responses (top row) to MPS responses determined from FE simulations using GHBMC (bottom row). Solid lines (red) indicate directionally dependent natural periods obtained from SDOF model fits to the uniaxial GHBMC data. 


\subsubsection{Correlations with Existing Metrics.}

When compared to existing rotational-based metrics, the sDOF model correlated better with strain-based responses using the 660 head impacts; $\mathrm{R}^{2}=0.955$ and 0.926 for MPS and CSDM, respectively (Figure 4-9, Table 4-3). For the subsets of uniaxial data, metrics that included at least two of the three kinematic variables (acceleration, velocity, duration) tended to perform better than metrics based on a single kinematic variable (Table 4-4). In addition to the sDOF model, RVCI and BRIC were some of the top performing metrics, each of which demonstrated some degree of dependence on both angular velocity and angular acceleration (Figure 4-10). Although correlations based on angular velocity were higher than angular acceleration, several metrics that included both angular velocity and angular acceleration had higher correlation with MPS and CSDM (Table 4-4).

Table 4-2: Fitted parameters for the uniaxial sDOF models.

\begin{tabular}{ccccccc}
\hline $\begin{array}{c}\text { Anatomical } \\
\text { direction }\end{array}$ & $n$ & $\begin{array}{c}\Delta t_{n} \\
(\mathrm{~ms})\end{array}$ & $\zeta$ & $\beta$ & $\begin{array}{c}\omega_{n} \\
(\mathrm{rad} / \mathrm{s})\end{array}$ & $\mathrm{R}^{2}$ \\
\hline$x$ & 173 & 36.2 & 0.525 & 2.76 & 173 & 0.986 \\
$y$ & 129 & 44.9 & 0.466 & 2.59 & 140 & 0.987 \\
$z$ & 147 & 38.4 & 0.416 & 4.01 & 164 & 0.984 \\
\hline
\end{tabular}

$n$ is the number of parametric cases in the subset used for model fitting.

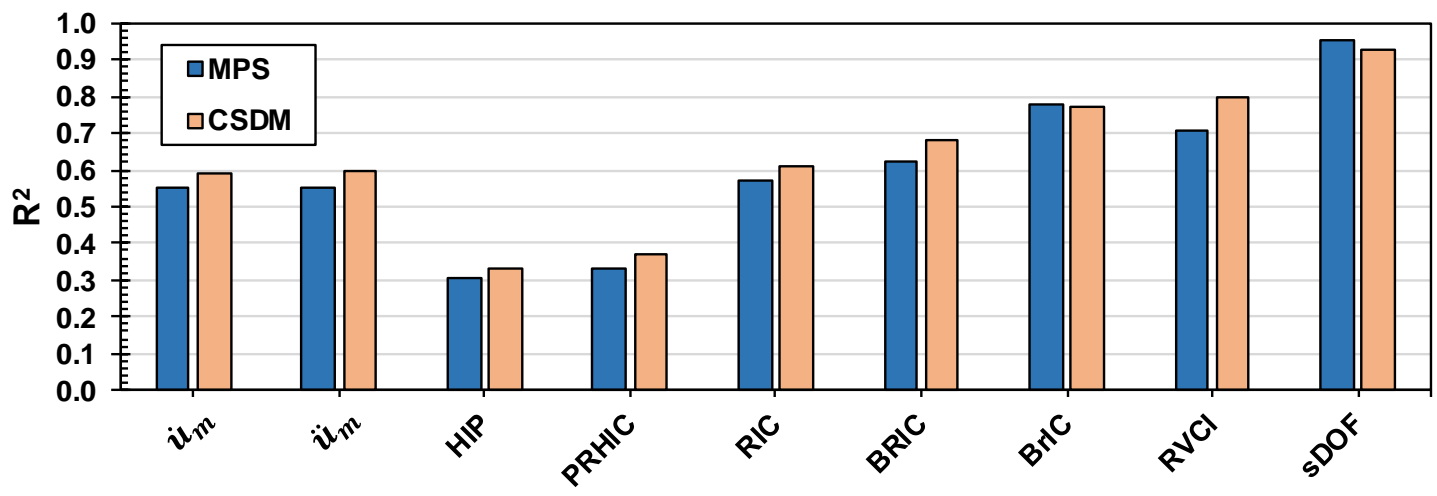

Figure 4-9: Correlations between existing rotational-based metrics and the SDOF model, and strain-based metrics obtained from GHBMC using the 660 head impacts from Chapter 3. Results for existing metrics are based on Figure 3-1. 


\subsection{Discussion}

Although numerous kinematic metrics have been proposed, many do not represent the underlying strain-based injury mechanism making them unsuitable for predicting brain injury over a broad range of head impacts. In this chapter, a sDOF model was developed to investigate the relationship between rotational head kinematics and brain deformation for development of improved forms of kinematic-based brain injury metrics. Efficacy of the sDOF model for predicting brain deformation was assessed through fits to FE-based maximum brain-strain response from a parametric study using idealized pulses covering a broad range of rotational head motions. Performance of the fitted sDOF model was evaluated relative to several existing rotational-based metrics using 660 real-world head impacts and a subset of the parametric data that had similar kinematic characteristics.
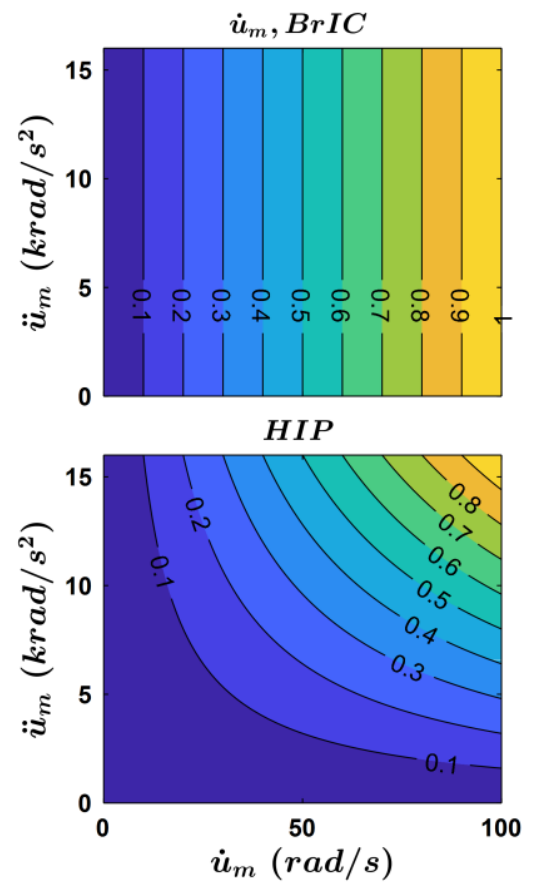

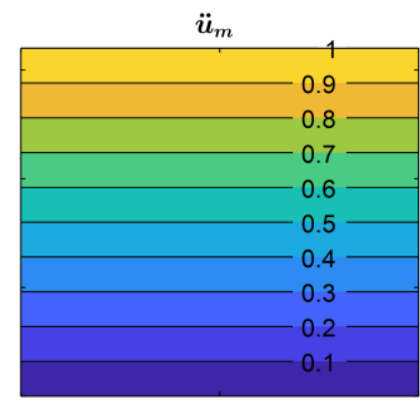

RVCI

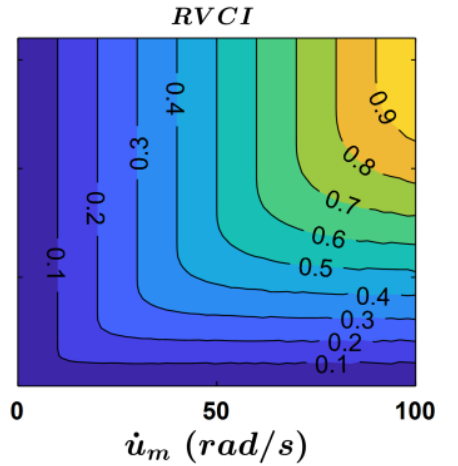

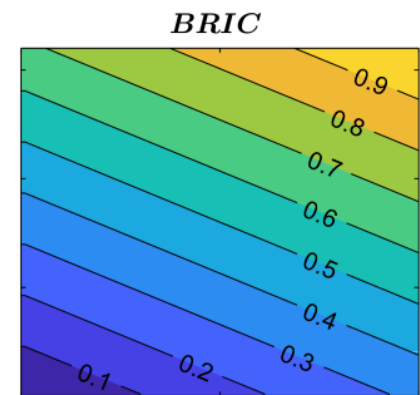

$s D O F$

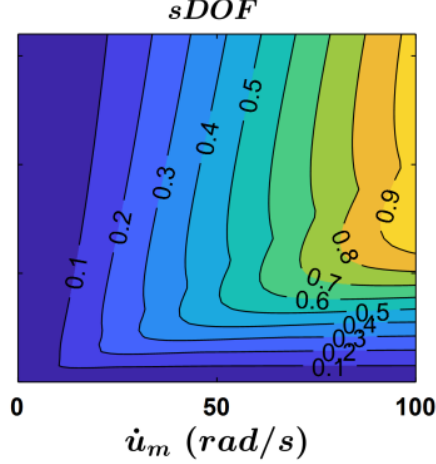

Figure 4-10: Contour plots for several existing kinematic-based metrics and the sDOF model. Contour lines indicate constant metric values which have been normalized by the maximum value within the plot. For directionally dependent metrics (BrIC, HIP, RVCI, and sDOF), only a single direction is shown. 


\subsubsection{Significance of the Results}

The similarities between sDOF and FE model maximum deformation responses from the parametric study, and their high correlation using the real-world data suggest that a simple mechanical system may be adequate to predict FE-based brain strain responses over a broad range of head impacts. This finding is important because it may allow for an analytical approach to understanding brain injury biomechanics due to rotational head motion. The relationship between head motion and maximum sDOF model displacement can be determined analytically for several idealized cases, which may help guide development of improved mathematical forms for future kinematic-based metrics. Furthermore, this work has the potential to significantly reduce computational costs associated with injury risk assessment using the far more complex FE models. Using a sDOF model, or a kinematics metric based on the maximum response of a sDOF model would provide a more feasible tool for rapid risk assessment since computation time is essentially instantaneous relative to the FE models.

Table 4-3: Regression results for 660 head impacts (Figure 4-9).

\begin{tabular}{|c|c|c|c|c|c|c|}
\hline \multirow[b]{2}{*}{ Metric } & \multicolumn{3}{|c|}{ MPS } & \multicolumn{3}{|c|}{ CSDM } \\
\hline & $\begin{array}{c}\text { Intercept } \\
\left(\beta_{0}\right)\end{array}$ & $\begin{array}{l}\text { Slope } \\
\left(\beta_{1}\right)\end{array}$ & $\mathrm{R}^{2}$ & $\begin{array}{c}\text { Intercept } \\
\left(\beta_{0}\right)\end{array}$ & $\begin{array}{l}\text { Slope } \\
\left(\beta_{1}\right)\end{array}$ & $\mathrm{R}^{2}$ \\
\hline$\dot{u}_{m}$ & $7.96 \mathrm{E}-2(9.82 \mathrm{E}-3)$ & $7.02 \mathrm{E}-3(2.46 \mathrm{E}-4)$ & 0.554 & $-1.75 \mathrm{E}-1(1.29 \mathrm{E}-2)$ & $9.95 \mathrm{E}-3(3.22 \mathrm{E}-4)$ & 0.593 \\
\hline$\ddot{u}_{m}$ & $1.78 \mathrm{E}-1(6.78 \mathrm{E}-3)$ & $4.52 \mathrm{E}-2(1.60 \mathrm{E}-3)$ & 0.549 & $-3.73 \mathrm{E}-2(8.80 \mathrm{E}-3)$ & $6.44 \mathrm{E}-2(2.07 \mathrm{E}-3)$ & 0.595 \\
\hline HIP & $-1.30 \mathrm{E}+3(1.23 \mathrm{E}+2)$ & $8.91 \mathrm{E}+3(3.34 \mathrm{E}+2)$ & 0.519 & $3.85 \mathrm{E}+2(6.11 \mathrm{E}+1)$ & $6.89 \mathrm{E}+3(2.27 \mathrm{E}+2)$ & 0.583 \\
\hline PRHIC & $3.09 \mathrm{E}-1(4.65 \mathrm{E}-3)$ & $1.82 \mathrm{E}-8(1.01 \mathrm{E}-9)$ & 0.328 & $1.49 \mathrm{E}-1(6.18 \mathrm{E}-3)$ & $2.64 \mathrm{E}-8(1.35 \mathrm{E}-9)$ & 0.367 \\
\hline RIC & $2.69 \mathrm{E}-1(4.22 \mathrm{E}-3)$ & $8.12 \mathrm{E}-9(2.76 \mathrm{E}-10)$ & 0.569 & $9.31 \mathrm{E}-2(5.51 \mathrm{E}-3)$ & $1.15 \mathrm{E}-8(3.60 \mathrm{E}-10)$ & 0.609 \\
\hline BRIC & $8.42 \mathrm{E}-2(8.44 \mathrm{E}-3)$ & $3.17 \mathrm{E}-1(9.60 \mathrm{E}-3)$ & 0.623 & $-1.72 \mathrm{E}-1(1.06 \mathrm{E}-2)$ & $4.54 \mathrm{E}-1(1.21 \mathrm{E}-2)$ & 0.682 \\
\hline BrIC & $-3.02 \mathrm{E}-5(7.53 \mathrm{E}-3)^{\mathrm{a}}$ & $4.62 \mathrm{E}-1(9.61 \mathrm{E}-3)$ & 0.778 & $-2.70 \mathrm{E}-1(1.04 \mathrm{E}-2)$ & $6.31 \mathrm{E}-1(1.33 \mathrm{E}-2)$ & 0.773 \\
\hline RVCI & $1.26 \mathrm{E}-1(6.08 \mathrm{E}-3)$ & $7.71 \mathrm{E}-3(1.93 \mathrm{E}-4)$ & 0.709 & $-1.45 \mathrm{E}-1(7.47 \mathrm{E}-3)$ & $9.42 \mathrm{E}-3(1.86 \mathrm{E}-4)$ & 0.797 \\
\hline $\mathrm{sDOF}$ & $4.55 \mathrm{E}-2(2.75 \mathrm{E}-3)$ & $8.19 \mathrm{E}-1(6.95 \mathrm{E}-3)$ & 0.955 & $-2.03 \mathrm{E}-1(4.82 \mathrm{E}-3)$ & $1.10 \mathrm{E}+0(1.22 \mathrm{E}-2)$ & 0.926 \\
\hline
\end{tabular}

Regression model parameter estimation (standard error).

${ }^{a}$ Indicates non-significant result $(\mathrm{p}>0.05)$. 
Using the mechanical models, maximum brain deformation magnitude was found to be governed by three general categories of rotational head motion, each distinguished by the duration of the pulse relative to the natural period of the brain. This governing behavior was observed in several previous studies (Gierke, 1964; Kornhauser and Lawton, 1961; Payne, 1962). In 1964, von Gierke categorized maximum sDOF model displacement into three regions of integral dependence based on the ratio of the pulse length to the natural period of the system: a region that depended on the integral of the pulse (short-duration), a region that depended on the pulse length (moderate duration), and a region that was independent of pulse length (long-duration) (Gierke, 1964). He also observed the maximum displacement of the sDOF model to be fairly insensitive to pulse shape. This finding suggests that the governing behavior between the magnitudes of velocity, acceleration, and deformation can be generalized for an arbitrary head impact pulse. In the current study, maximum deformation in the mechanical models had some dependence on angular acceleration in short duration, and angular velocity in long duration (contours of maximum deformation were not perfectly vertical or horizontal; Figures 4-4 and 4-5). This is due to the use of a full cycle pulses where von Gierke only used half cycle pulses.

Alarmingly, much of the sled, crash, and pendulum data were concentrated in the region near the natural period where brain deformation depended on both angular velocity and angular acceleration. This suggests that these impact environments may lead to amplified brain deformations and hence increased injury risk due to the resonance behavior of the brain. This finding agrees with previous work, which found head impacts occurring in American football to excite the resonance frequency of the brain-skull system (Laksari et al., 2015). Given the large number of TBIs that are occurring in these impact 
environments further indicates the need to include both angular velocity and angular acceleration in the mathematical form of brain injury criteria.

Table 4-4: Regression results for the uniaxial subsets.

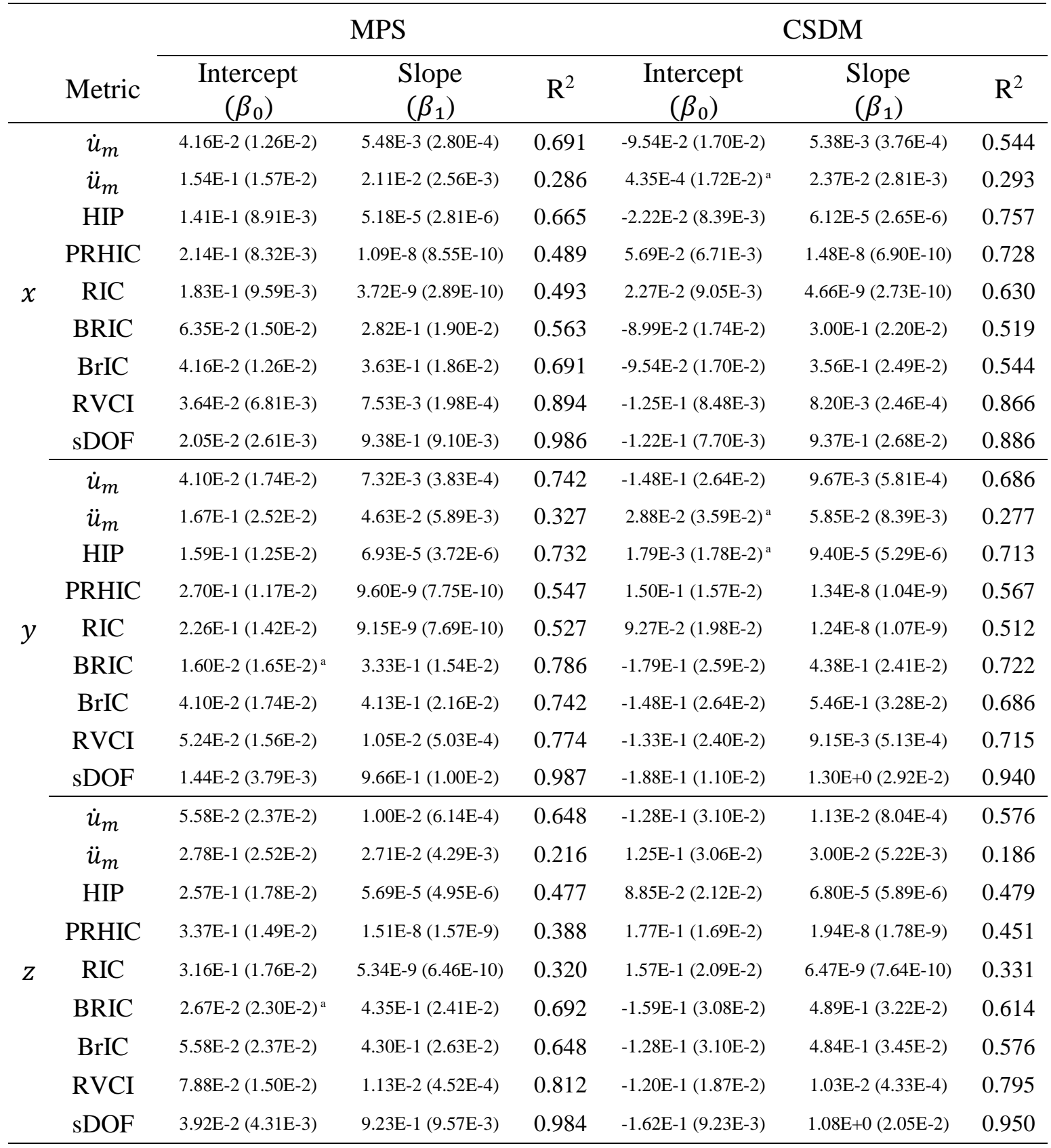

Regression model parameter estimation (standard error).

${ }^{\mathrm{a}}$ Indicates non-significant result $(\mathrm{p}>0.05)$. 
Response patterns observed in the mechanical models also showed remarkable similarity to several experimentally derived rotational brain injury tolerances proposed in the literature (Gennarelli et al., 1985; Margulies and Thibault, 1992; Ommaya and Hirsch, 1971). In these experimental studies, brain injury tolerances show regions of dependence on angular velocity only and angular acceleration only in a manner that is consistent with the maximum deformation responses from mechanical models (Figure 4-11). Thus, the current study provides additional evidence that tissue-level brain strain is the mechanism for closed-head induced brain injury, and suggests that the link between rotational head kinematics and brain injury responses may be adequately explained using fundamental mechanical models. Future work in this area should focus on linking deformation-based response patterns obtained from the mechanical models to brain injury risk in humans.

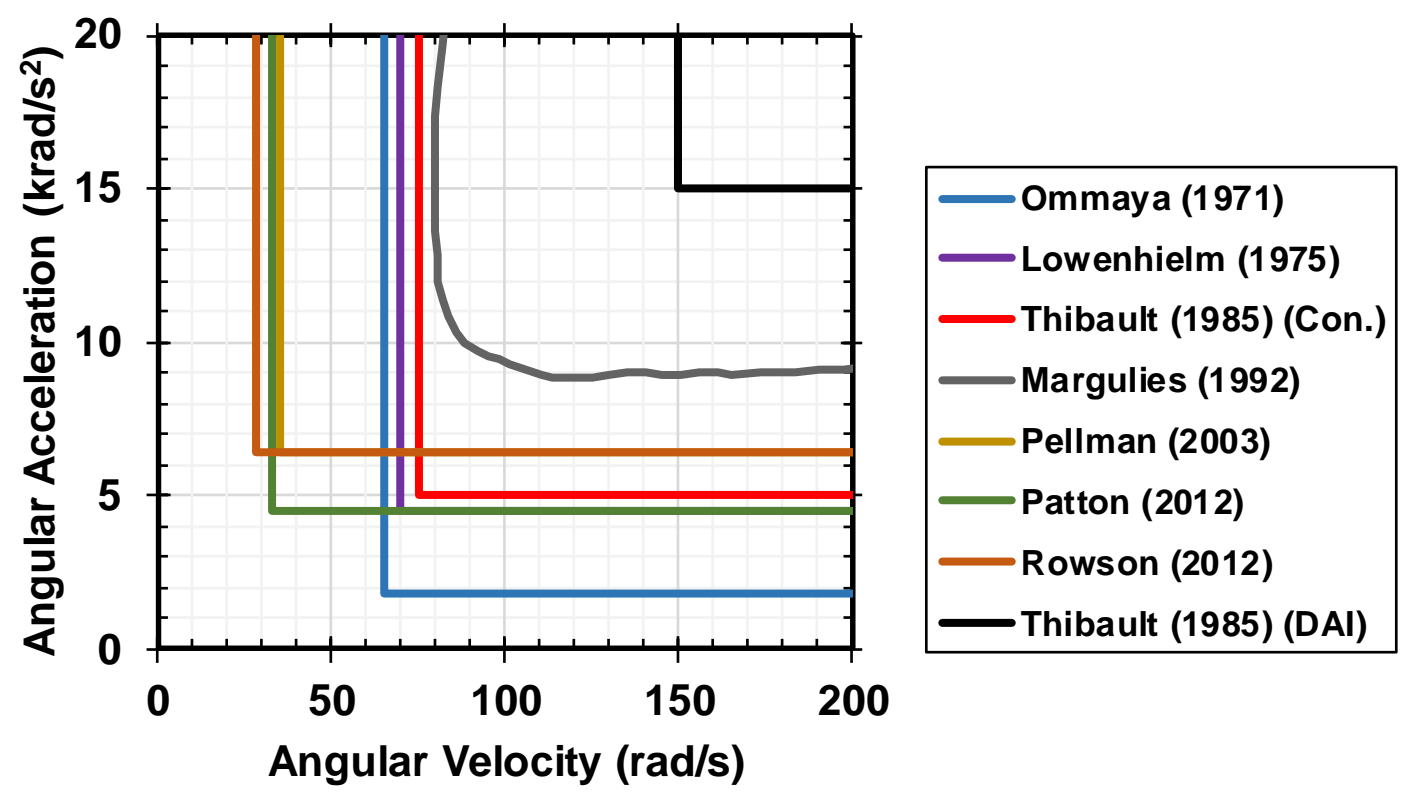

Figure 4-11: Experimental human tolerances for various brain injury types including concussion and DAI. While the values of the tolerances differ, they have a similar trend (corner shape) to the deformation patterns seen in the contour plots for the SDOF and FE models, respectively (Figures 4-8). 
The current study may also explain why some brain injury tolerances have been based on either angular velocity only or angular acceleration only. Direct head impacts typically result in short-duration acceleration pulses where brain deformation is insensitive to the magnitude of acceleration and more consistent with the change in angular velocity (Ommaya and Hirsch, 1971; Takhounts et al., 2013). Indirect head impacts would typically result in longer duration pulses where deformation is insensitive to the change in velocity and more consistent with the magnitude of acceleration (Gennarelli et al., 1985; Yanaoka et al., 2015). Thus, these behaviors suggest that future brain injury metrics be developed with datasets covering a broad range of kinematic magnitudes and durations to prevent development of brain injury criteria on angular velocity or angular acceleration alone.

\subsubsection{Limitations with Existing Rotational Metrics}

Relative to existing rotational-based metrics, the sDOF model performed better using real-world impacts and idealized head motions; correlations with MPS and CSDM were highest with the sDOF model (Figure 4-9, Tables 4-3 and 4-4). Although several other rotational metrics correlated well with strain-based responses, many of these metrics do not capture the underlying physics of brain deformation for a broad range of durations.

RVCI correlated well with strain-based responses from the uniaxial simulations, and exhibited similar response characteristics to the mechanical models (Figure 4-10). This finding is not surprising given that RVCI was based on deformation from a spring-mass system (Yanaoka et al., 2015). However, there are several assumptions associated with the development of RVCI which may limit its performance as a brain injury criterion. The effect of damping on the maximum magnitude of displacement of the spring-mass system was not investigated, which could result in RVCI overestimating maximum brain 
deformation for a given magnitude of velocity and acceleration. Additionally, due to simplifications through a mathematical approximation, RVCI would underestimate deformation near system resonance, a critical region that contains real-world head impacts and amplified brain deformations. Finally, RVCI addresses the transition between angular velocity and angular acceleration dependence through an ad-hoc procedure that involves finding the maximum value from a time history integration that uses arbitrary limits in a manner similar to HIC. Although RVCI has demonstrated improved predicative capability over many of existing kinematic-based metrics, future work should focus on developing rotational metrics from more rigorous analysis of the underdamped sDOF system.

Response surfaces for other metrics including HIP, RIC, PRHIC, and BRIC demonstrated some dependence on both angular velocity and angular acceleration; however, these metrics fundamentally do not relate rotational head kinematics to brain deformation. HIP is based on the maximum rate of change of kinetic energy entering the head during an impact (Newman et al., 2000). It is possible that an energy based criterion is a good predictor of brain injury; however additional experimental work should be completed to verify this hypothesis. PRHIC and RIC, which behaved very similar to HIP (Figure 4-10), and BRIC are empirically derived formulations that are considered to be measures of head impact severity, and do not relate to fundamental mechanics principles.

Although BrIC was previously shown to correlate well with MPS and CSDM for a majority of the sled, crash, and pendulum data; Chapter 3 (Takhounts et al., 2013), it can only be used effectively for shorter-to-moderate duration impacts. Since BrIC is based on angular velocity alone (vertical contour lines), it will over-estimate brain strain for moderate- and long-duration events. Results have shown low correlation between BrIC and 
strain-based metrics in high velocity/low acceleration (long-duration) events; Chapter 3 (Yanaoka et al., 2015). In a previous parametric study using SIMon, brain strain decreased with increasing pulse duration beyond 50ms for a constant magnitude of angular velocity (constant BrIC) (Gabler et al., 2016). In Chapter 3, BrIC was found to have relatively low correlation with MPS and CSDM in pedestrian impacts (Figure $3-3, \mathrm{C}$ and D; $\mathrm{R}^{2}=0.4-$ 0.5), which are typically higher velocity, lower acceleration events (before head impact), compared to occupant crash modes (Figure 3-3, A and B, and C; $\mathrm{R}^{2}=0.6-0.8$ ). The location of the sled, crash, and pendulum data relative to the natural period of the FE models suggests that some real-world head impacts fall in the longer duration region where brain strain depends on angular acceleration (horizontal contour lines). Although the critical values of BrIC could be re-tuned to give a better estimation of strain for a subset of impact durations, BrIC fundamentally cannot predict brain strains for head impacts covering a broad range of durations.

Furthermore, implementation of improved safety countermeasures will likely shift head kinematic responses to longer durations, by reducing the magnitude of head angular acceleration during impact. Although angular velocity may be reduced simultaneously, if the impact shifts to an acceleration-only depended region, then a criterion based on angular velocity alone, may be insensitive to countermeasure improvements. In the current study, estimates for the natural periods for the GHBMC-based MPS response were between 36 and $45 \mathrm{~ms}$, depending on direction. Since these values roughly indicate the transition of brain strain dependence from angular velocity to angular acceleration, values for the natural period may be used to establish limits of applicability for certain brain injury metrics. For instance, BrIC (an angular velocity metric) would be best suited for short-duration impacts; 
below the natural period of the brain, and angular acceleration metrics would be best suited for long-duration impacts; above the natural period of the brain. A metric combining angular velocity and acceleration would be needed to assess head impacts of all types.

\subsubsection{Limitations of the Current Study}

Efficacy of sDOF model for predicting brain injury relies heavily on the assumption that the FE models are predictive tools. While both FE models have been validated for various intracranial responses including brain deformation, angular head motions for some of the sled, crash, and pendulum impacts fall outside the range of the experimental data used to validate these models (Chapter 3; Discussion). Although pulse durations for the parametric study were varied from $1-3000 \mathrm{~ms}$, sDOF model fits and regression analyses were performed using a subset of the data $(6-157) \mathrm{ms}$, which included uniaxial cases that had similar magnitudes and durations to the 660 head impacts (Table 4-1). Head motions at longer and shorter durations, and high magnitudes, i.e., motions for which the FE models have not been validated, or which may cause other types of injury (skull fracture), were used only for qualitatively comparing deformation response patterns with the sDOF model.

Although the sDOF model showed efficacy for predicting FE-based strain responses, additional work may be necessary to clarify the effect of more complicated head motions on the development of brain strains. In this study, only single, uniaxial pulses with a sinusoidal shape were considered. Sinusoidal pulses have typically been used to parametrize arbitrary head impact kinematics; however, real-world impacts often result in complex, multi-impact head motions involving both translational and rotational components with eccentric load vectors. Although previous studies have shown translational kinematics to be poor predictors of FE-based strain responses, (Kleiven, 2007; 
Takhounts et al., 2013) the effect of multi-impact, coupled head motions on the development of brain strain should be investigated in the future.

Only global measures of brain strain were used in this study. While evidence suggests that regional tissue responses may be better indicators of focal injury (Elkin and Morrison III, 2007; Hernandez et al., 2015; Sullivan et al., 2015), additional work is needed to determine whether metrics based on regional tissue strain improve the prediction of these brain injury types. Additionally, several studies suggest that metrics based on strain rate, the product of strain and strain rate, or tract-orientated strain may improve correlation with brain injury; e.g., (King et al., 2003; Sullivan et al., 2015; Zhao et al., 2017). While results from these studies are encouraging, their findings are based either on animal models which require scaling, or human impact reconstructions which have not been sufficiently scrutinized for accuracy of their angular head motions and subsequent use in FE brain models. Therefore, additional work is necessary to determine whether these metrics improve prediction of brain injury in humans. Although the sDOF models developed in the current study do not account for tissue fiber orientation, their rate of displacement could be used to estimate brain tissue strain rates for future research purposes.

Currently, the sDOF model can only be used to estimate MPS for a single brain element; $95^{\text {th }}$ percentile MPS, which may be insufficient for predicting brain injuries that occur under more complex strain distributions. While the system parameters of the sDOF model could be tuned to estimate maximum principal brain strain for other ranked elements, a more robust approach involving multiple spring-mass-damped elements, i.e., a multibody model is recommended. Multibody models could be used to rapidly estimate brain deformation across various regions of the whole brain allowing for localized strain 
predictions. Other approaches that are capable of estimating whole-brain strains during head impact involve pre-computation (Ji and Zhao, 2015; Zhao and Ji, 2016b; Zhao et al., 2017). While this technique offers an additional cost-effective approach to FE model simulation, brain strain estimates are determined from pre-computed values based on pulse simplification algorithms, which do not address the fundamental mechanics of the problem, and have not be verified for a broad range of head impact conditions.

\subsubsection{Comments on Numerical Simulations}

Simulations using the FE and sDOF models were stable for most of the parametric cases. Model instabilities did occur at very long durations $(\Delta t>1100 \mathrm{~ms}$ ), which caused early termination of several FE simulations; however, these cases were not used in the sDOF model fits. While deformation patterns between GHBMC and SIMon were similar for short-to-moderate duration uniaxial pulses, SIMon behaved more erratic at longer durations, and the transition between velocity and acceleration dependent deformations was not as distinct as GHBMC and the sDOF model. Currently there is no experimental data available to confirm that one model is more correct than the other, thus the accuracy of FE model brain strain response under longer duration head motions should be assessed in future studies. Using the SDOF model, there were several cases in which specific combinations of system parameters (typically involving lower damping ratios) resulted in inflections in the response surface (Figure 4-4). These inflections were characterized by a discontinuity in the slope of the contours, which was due to the maximum displacement occurring at secondary peaks in the time history (Appendix F). 


\subsection{Conclusions}

A sDOF model was developed and assessed for predicting strain-based brain injury responses through an extensive parametric study involving application of idealized rotational kinematic pulses to two different FE brain models. Results show that maximum brain deformation in the sDOF and FE models was governed by a nonlinear relationship between angular velocity and angular acceleration. This governing behavior also showed

remarkable similarity to previously derived experimental tolerances for brain injury demonstrating the efficacy of a simple mechanical system for brain injury risk assessment in a broad range of head impact environments. Furthermore, the mathematical forms of most existing kinematic brain injury criteria are fundamentally incapable of predicting brain strain response over a broad range of head impacts. This finding combined with the observation that typical TBI-inducing head impacts may potentially lead to increased brain injury risk due to resonance of the brain highlights the need for improved mathematical forms for brain injury criteria that are based on both angular velocity and angular acceleration. Doing so will improve prediction of brain injury over a broad range of head impacts, and help to discriminate the efficacy of improved safety systems. 


\section{Chapter 5 - Universal Brain Injury Criterion}

Diffuse brain injuries are caused by excessive brain deformation generated primarily by rapid rotational head motion. Metrics that describe the severity of brain injury based on head motion often do not represent the governing physics of brain deformation, rendering them ineffective over a broad range of head impacts. This chapter presents the development of a new brain injury metric that is based on the response of a second-order mechanical system, and relates rotational head kinematics to strain-based injury metrics from the FE human brain models. This chapter has been submitted for publication as a manuscript (Gabler et al., 2017. Development of an Injury Metric for Predicting Brain Strain Responses using Head Kinematics. Annals of Biomedical Engineering).

\subsection{Introduction}

In the previous two chapters, limitations with existing kinematic-based metrics for predicting brain strain response were elucidated. In Chapter 3, it was found that metrics based on rotational head kinematics correlated better with brain strain responses than those based on translational kinematics, over a broad range of impacts. This finding was not surprising given that brain tissue is nearly incompressible, and shear strains are caused primarily by rotational head motion. Metrics based on angular velocity had the highest correlation out of those evaluated; however, their performance was limited in several impact conditions, namely in long duration events. Results from Chapter 4 showed remarkable similarity between the deformation patterns of a sDOF model and both FE brain models. This suggests that maximum brain strain under rotational head motion can be adequately represented by maximum deformation from a second-order mechanical system under excitation. Furthermore, maximum brain strain depended on the magnitudes of 
angular velocity and angular acceleration, and their relationship to the brain's natural period. Although correlations between maximum brain strain and metrics based on a combination of angular velocity and angular acceleration were higher than those based on velocity or acceleration alone, they do not relate rotational head kinematics to brain deformation on a fundamental mechanics level.

This study focuses on the development of a new kinematic-based metric called the Universal Brain Injury Criterion (UBrIC), which is formulated based on the governing relationship between excitation and maximum deformation of a second-order system. The universal term of UBrIC represents the applicability of this metric to a broad range of impact conditions including automotive and sport. Efficacy of UBrIC for predicting strainbased responses from FE brain models was investigated relative to existing kinematicbased metrics using a broad range of head impacts. This study highlights the advantage of using a physics-based metric for predicting brain strain response using head kinematics in a broad range of impact conditions, and provides a tool for assessing the safety of helmets and automotive countermeasures.

\subsection{Methods}

\subsubsection{Development of UBrIC}

UBrIC is based on the assumption that maximum brain deformation under rotational head motion is analogous to deformation from a second-order system under excitation. In Chapter 4, a sDOF model was used to show that brain deformation in one dimension is governed by three general categories of rotational head motion, each distinguished by the pulse duration $(\Delta t)$ relative to the natural period $\left(\Delta t_{n}\right)$ of the brainskull system: for short-duration pulses, maximum brain strain depended primarily on the 
magnitude of angular velocity (Figure 5-1, A; $\Delta t \rightarrow \Delta t_{1}$ ), for long-duration pulses, maximum brain strain depended primarily on the magnitude of angular acceleration (Figure $5-1, \mathrm{~A} ; \Delta t \rightarrow \Delta t_{2}$ ), and for pulses near the natural period of the brain, maximum strain depended on the magnitudes of velocity and acceleration (Figure 5-1, A; $\Delta t \rightarrow \Delta t_{n}$ ).

(A)

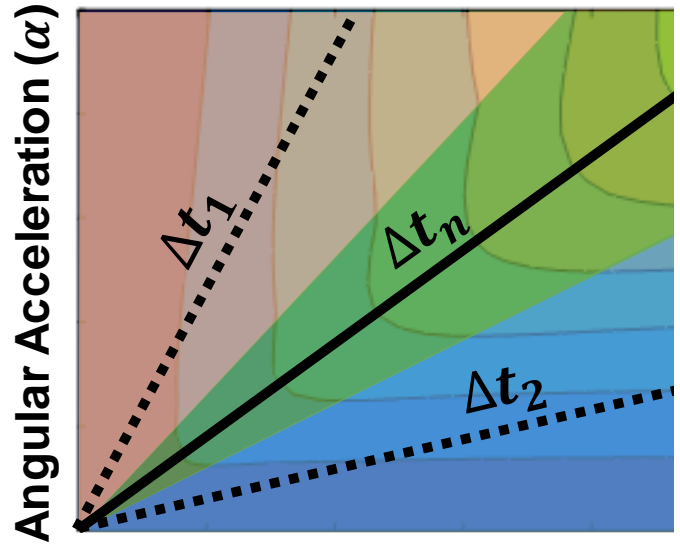

Angular Velocity $(\omega)$
(B)

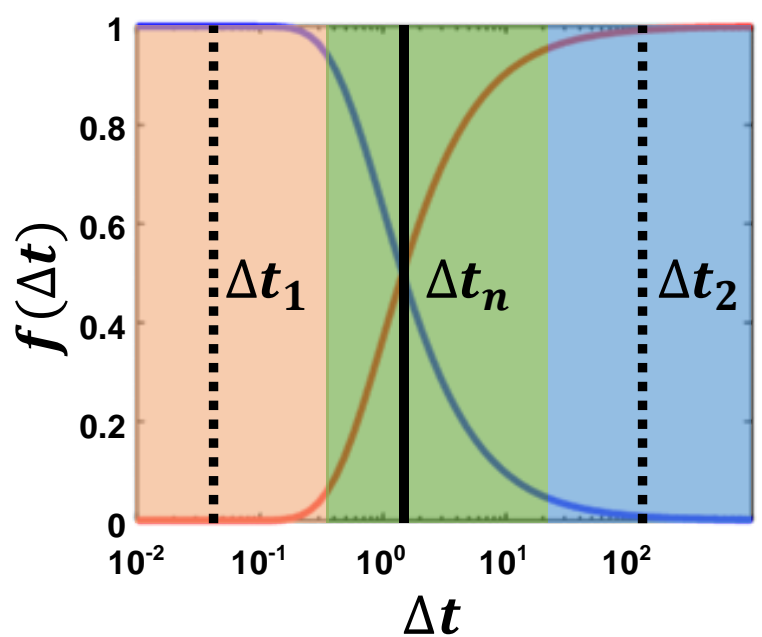

Figure 5-1: Contours of maximum deformation from the SDOF model subjected to a broad range of angular velocity and angular acceleration magnitudes $(\mathrm{A})$, where $\Delta t_{1}<\Delta t_{n}<\Delta t_{2}$. The contour plot was obtained from Figure 4-8, which share remarkable similarity to the maximum principal strain contours from $\mathrm{FE}$ brain models. Exponential functions that were used to generalize the deformation behavior of the SDOF and FE brain models (B).

To generalize the transition between velocity and acceleration dependent brain strains for a one dimensional impact pulse, exponential functions were used (Figure 5-1, B). Adding these exponentials resulted in a function that switches between velocity and acceleration dependent deformations in a manner creating a velocity-only dependence in short-duration $(f \rightarrow \omega$ as $\Delta t \rightarrow 0)$, and acceleration-only dependence in long-duration, $(f \rightarrow \alpha$ as $\Delta t \rightarrow \infty):$

$f(\Delta t)=\omega\left(1-e^{-\frac{1}{\Delta t}}\right)+\alpha e^{-\frac{1}{\Delta t}}$, Equation 5-1 
where $f$ is a functional that establishes brain deformation given the magnitudes of angular velocity $(\omega)$ and angular acceleration $(\alpha)$, and duration of a one dimensional head impact pulse. Assuming that the duration of an arbitrary impact is related to the magnitudes of angular velocity and acceleration, $\Delta t=\omega / \alpha$, and that the one dimensional deformation can be generalized for rotations about each axis of the head, the following kinematic-based metric is proposed:

UBrIC $=\left\{\sum_{i}\left[\omega_{i}^{*}+\left(\alpha_{i}^{*}-\omega_{i}^{*}\right) e^{-\frac{\alpha_{i}^{*}}{\omega_{i}^{*}}}\right]^{r}\right\}^{\frac{1}{r}}$,

Equation 5-2

where $\omega_{i}^{*}$ and $\alpha_{i}^{*}$ are the directionally dependent $(i=x, y, z)$ maximum magnitudes of head angular velocity and angular acceleration each normalized by a critical value $(c r) ; \omega_{i}^{*}=$ $\omega_{i} / \omega_{i c r}$ and $\alpha_{i}^{*}=\alpha_{i} / \alpha_{i c r}$. The critical values normalize the metric to maximum brain strain and control the transition between velocity and acceleration dependent deformations. The exponent $r$ establishes the power at which the magnitude is evaluated; model performance was assessed for $r$ equals one and two. Six total parameters (two critical values per direction) were used to establish the full three dimensional form of UBrIC.

\subsubsection{Additional Kinematic Forms}

In addition to UBrIC, several mathematical forms based on existing rotational metrics were assessed for predicting brain strain responses. These metrics are based on the maximum (resultant or directionally dependent) magnitudes of angular velocity and angular acceleration, and were included in the analysis to benchmark improvement using UBrIC with data from the current study (Table 5-1). Metrics based on translational kinematic parameters were not included, since they were shown to have poor correlation with strain-based metrics, and contribute to negligible strain (Chapter 3). 
Two fundamental kinematic parameters used in brain injury criteria are the maximum values of the resultant angular velocity and angular acceleration:

$z_{m}^{*}=\frac{z_{m}}{z_{m c r}}$

Equation 5-3

where $\boldsymbol{z}$ is equal to $\boldsymbol{\alpha}$ or $\boldsymbol{\omega}$, and $z_{m}$ is maximum resultant kinematic variable taken over the time history (Equation 3-1). Linear combinations of the directionally dependent maximum magnitudes of angular velocity and angular acceleration were also included:

$z_{i}^{*}=\left\{\sum_{i} z_{i}^{* r}\right\}^{\frac{1}{r}}$

Equation 5-4

Where $z$ indicates $\omega$ or $\alpha$, and performance was assessed for $r$ equals one and two. Equation 5-4 with $z=\omega$ and $r=2$ represents BrIC (Takhounts et al., 2013). In this chapter, Equation 5-4 is referred to as BrIC (refit) since the critical values of BrIC for were refit using data from Chapter 2 to compare it equally with UBrIC. The original BrIC was also included in the analysis where $\omega_{x c r}=66.25 \mathrm{rad} / \mathrm{s}, \omega_{y c r}=56.45 \mathrm{rad} / \mathrm{s}$, and $\omega_{z c r}=42.87 \mathrm{rad} / \mathrm{s}($ Table $3-2)$. Metrics that consisted of angular velocity and angular acceleration were also included in this chapter. Mathematical forms based on a linear combination of the maximum resultant (Equation 5-5) and directionally dependent (Equation 5-6) magnitudes were investigated:

$C_{m}=\omega_{m}^{*}+\alpha_{m}^{*}$,

Equation 5-5

and

$C_{i}=\left\{\sum_{i}\left[\omega_{i}^{*}+\alpha_{i}^{*}\right]^{r}\right\}^{\frac{1}{r}}$

Equation 5-6

where subscripts $m$ and $i$ indicate the resultant and directionally dependent forms for the combination $(C)$, respectively; Equation 5-5 is based on BRIC (Takhounts et al., 2011) 
Table 5-1: Kinematic-based metrics used in this chapter.

\begin{tabular}{lccccc}
\hline Metric & $\begin{array}{c}\text { \# of } \\
\text { critical } \\
\text { values } \\
\left(n_{p}\right)\end{array}$ & $\begin{array}{c}\text { Directional } \\
\text { dependence } \\
(i)\end{array}$ & $\begin{array}{c}\text { Angular } \\
\text { velocity } \\
\text { type }\end{array}$ & $\begin{array}{c}\text { Values } \\
\text { for } r\end{array}$ & $\begin{array}{c}\text { Total \# of } \\
\text { models fit }\end{array}$ \\
\hline$\omega_{m}^{*}$ (Equation 5-3) & 1 & No & NA & NA & 1 \\
$\alpha_{m}^{*}$ (Equation 5-3) & 1 & No & NA & NA & 1 \\
$C_{m}$ (Equation 5-5) & 2 & No & NA & NA & 1 \\
$\omega_{i}^{*}$ (Equation 5-4) ${ }^{\text {a }}$ & 3 & Yes & peak, p2p & 1,2 & 4 \\
$\alpha_{i}^{*}$ (Equation 5-4) & 3 & Yes & NA & 1,2 & 2 \\
$C_{i}($ Equation 5-6) & 6 & Yes & peak, p2p & 1,2 & 4 \\
UBrIC (Equation 5-2) & 6 & Yes & peak, p2p & 1,2 & 4 \\
\hline
\end{tabular}

${ }^{a} \mathrm{BrIC}$ (refit) is $r=2$; Not Applicable (NA).

\subsubsection{Head Impact Data}

A total of 1595 head impacts from the database were selected to fit the critical values of the metrics listed in Table 5-1. These data include all head impacts from the sled, and impactor categories, and most of the crash tests (Table 2-1). Head impacts from NHTSA's OMDB $(n=130)$ and RD full frontal rigid barrier $(n=22)$ crash test modes were not included in the fit, and instead used as an independent dataset for assessing the performance of the fitted metrics. Kinematics were processed based on methods described in Chapter 2; Angular acceleration magnitudes were calculated by taking the maximum resultant or directionally dependent magnitudes evaluated over the entire event time history (Equation 3-1). Angular velocity magnitudes were calculated two separate ways by taking:

(1) the maximum magnitudes (Equation 3-1, peak model), and (2) the difference between maximum and minimum values (Equation 5-7, peak-to-peak; $p 2 p$ model) in each anatomical direction over the entire event:

$\omega_{i}=\max _{t}\left\{\omega_{i}(t)\right\}-\min _{t}\left\{\omega_{i}(t)\right\}$.

Equation 5-7 
The peak-to-peak model was included since deformation from the second-order system was shown to depend on the integral of the pulse (maximum velocity change) in short duration (Equation F-31).

\subsubsection{Model Fitting and Statistics}

The critical values for each kinematic-based metric were determined through fits to strain-based metrics obtained from FE simulation of the 1595 head impacts. A nonlinear, least-squares solver (lsqcurvefit; Matlab, v8.4.0, The MathWorks, Natick, Ma) was used to minimize the SSE between kinematic metric-predicted and FE model-measured MPS and CSDM. For CSDM-based fits, an intercept parameter $\beta_{0}$ was added to each metric (outside the square root), and included in the fit. The intercept was used to correct for cases in which non-zero head kinematic responses resulted in zero FE model-based CSDM due to the MPS threshold; metrics were constrained to positive values only, e.g., if UBrIC $<0$, then $\mathrm{UBrIC}=0$. A total of 102 model fits were performed; seventeen kinematic-based metrics fit to three strain-based metrics from two FE brain models.

Model fits were assessed using the coefficient of determination (Equation 3-16) and the normalized root mean square error (NRMSE). Correlations between kinematic metricpredicted $\left(\widehat{Y}_{j}\right)$ and FE model-measured $\left(Y_{j}\right)$ MPS and CSDM values were adjusted for the number of critical values $\left(n_{p}\right)$ and samples $(n)$ used (Milton and Arnold, 2002):

$R_{a d j}^{2}=1-\frac{\left(1-R^{2}\right)(n-1)}{\left(n-n_{p}-1\right)}$

Equation 5-8

Since correlations can only be used to evaluate association between metrics, the NRMSE was used to assess the accuracy of the kinematic metric-prediction of MPS and CSDM:

$$
N R M S E=\sqrt{\frac{M S E\left(\hat{Y}_{j}\right)}{M S E\left(Y_{t}\right)}},
$$

Equation 5-9 
where $\operatorname{MSE}(\tilde{u})=\frac{\sum_{j=1}^{n}\left(Y_{j}-\widetilde{u}\right)^{2}}{\left(n-n_{p}-1\right)}$.

Equation 5-10

and $\tilde{u}$ is a dummy variable for the $j^{\text {th }}$ kinematic metric-prediction, $\hat{Y}_{j}$, and the null model, $Y_{t}$, which is the average FE model-based MPS or CSDM used in the fit. The NRMSE is a statistical metric used to evaluate the predictive performance of a regression model relative to a minimum information model; Null model; $n_{p}=0$ (Lobo et al., 2015). In this dissertation, 1-NRMSE values are reported to keep consistency with $\mathrm{R}^{2}$, where higher values indicate better model accuracy.

\subsubsection{Model Performance Assessments}

The relative performance of the fitted kinematic-based metrics was evaluated in multiple ways: First, an overall assessment was performed by comparing correlations $\left(\mathrm{R}^{2}\right)$ and metric-accuracy (1-NRMSE) from fits using the full head impact database $(n=1595)$ and the metrics in Table 5-1. Based on this assessment, the top performing metrics were selected and compared using the impact conditions listed in Table 2-1. Second, idealized rotational head motions from Chapter 4 were used to qualitatively assess response patterns between the top metrics and strain-based responses. Finally, head impacts from NHTSA's

OMDB and full frontal rigid barrier crash test modes were used as an independent dataset (not used in the fit) for assessing the relative performance of the fitted models.

\subsection{Results}

\subsubsection{Overall Assessment}

Relative to other metrics, UBrIC had the best overall performance with the database; for the peak-to-peak model with $r=2, \mathrm{R}^{2}=0.931,0.895$ and 1 -NRMSE $=0.736$, 0.675 for MPS and CSDM, respectively were the highest reported (Figure 5-2). From the 
additional forms considered, $C_{i}$ with $r=2$ was among the top performing metrics; $\mathrm{R}^{2}$ and 1-NRMSE were similar, but slightly lower than UBrIC. Fits with BrIC (refit) were better than the directionally dependent angular acceleration form, $\alpha_{i}^{*}$, but were lower than UBrIC and $C_{i}$. Furthermore, BrIC (refit) and $C_{i}$ systematically over-predicted strain-based responses for lower severity impacts (e.g., MPS; Figure 5-3 top and middle left graphs).

(A) Correlations with database

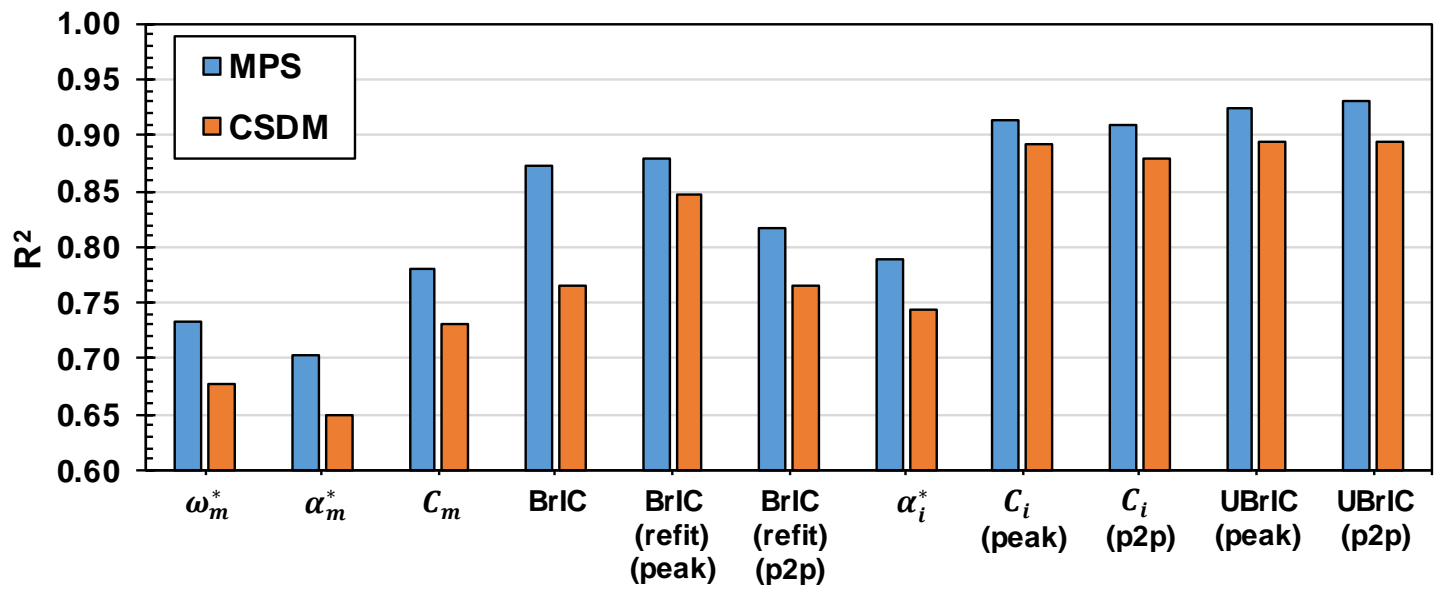

(B) Metric-accuracy with database

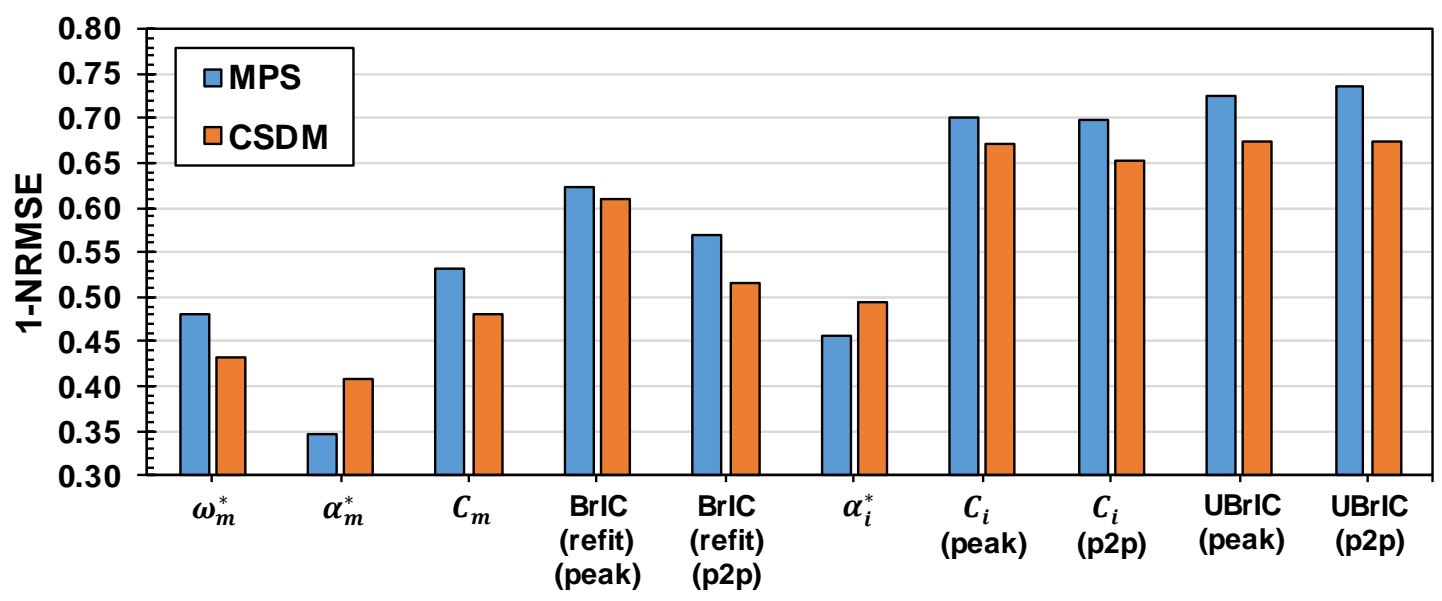

Figure 5-2: Correlations (A) and metric-accuracy (B) for kinematic-to-strain metric fits from GHBMC using 1595 head impacts. Results are shown for metrics with $r=2$ only. MPS is based on the $95^{\text {th }}$ percentile value. BrIC was not included in the metric-accuracy assessment because its values do not correspond to MPS and CSDM from GHBMC. 
BrIC (refit, peak)
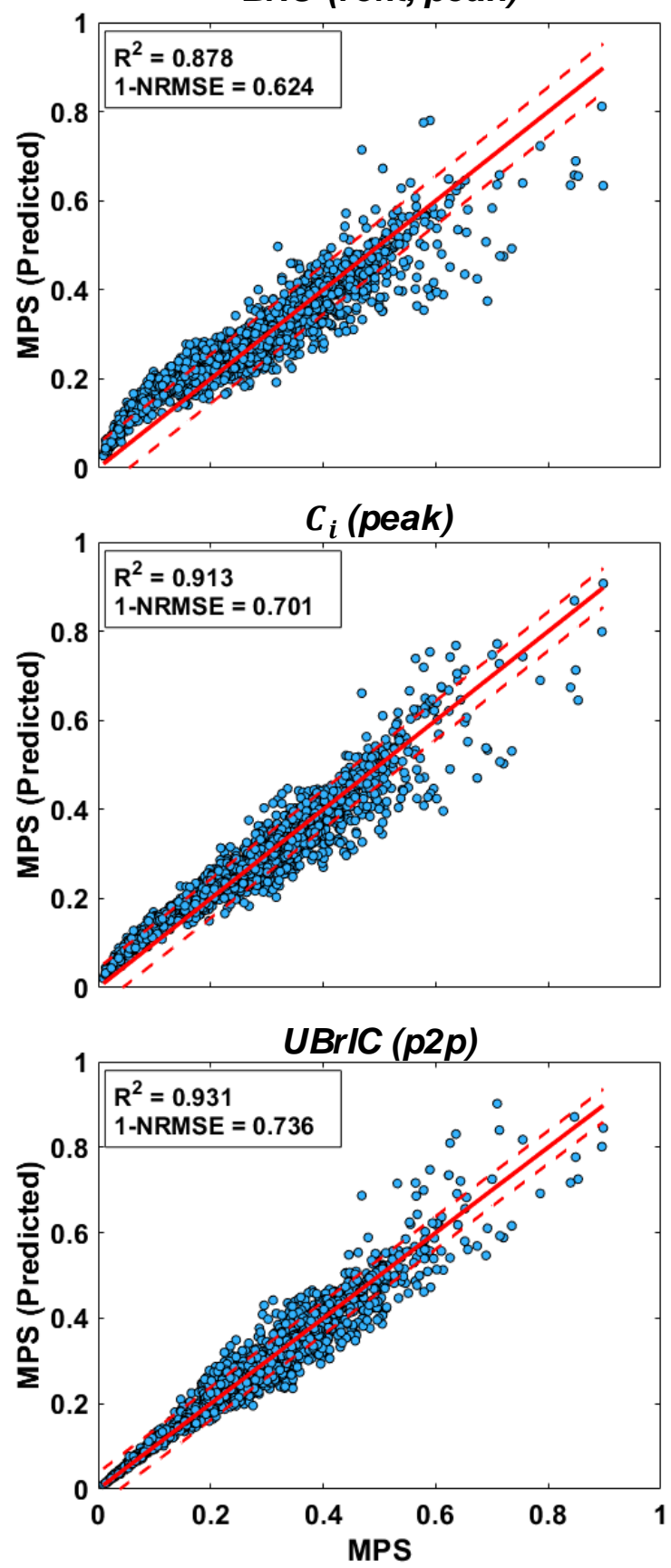

BrIC (refit, peak)
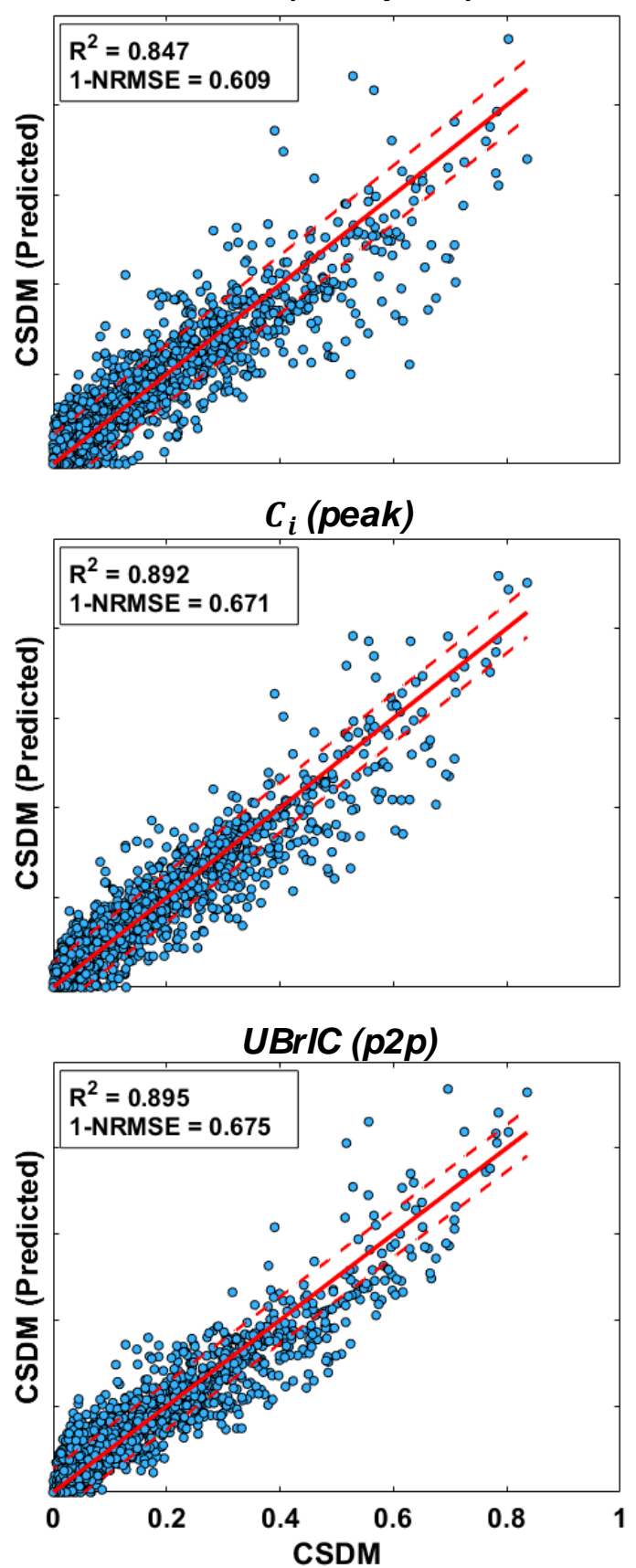

Figure 5-3: Scatter plots for top performing fitted metrics to MPS (left column) and CSDM (right column) using 1595 head impacts. MPS is based on the $\mathbf{9 5}^{\text {th }}$ percentile value. Solid red lines indicate a one-to-one relationship, while dotted red lines are \pm 1 root mean square error. Results shown are for metrics with $r=2$.

In general, metrics with $r=2$ performed better than $r=1$, while peak angular velocity forms performed better than those based on the peak-to-peak except for UBrIC. 
Metrics based on resultant kinematics were lower than directionally dependent forms; however, $\mathrm{R}^{2}>0.6$ for all model fits (Figure 5-2, A). When compared to CSDM-based fits, correlations and metric-accuracy were higher with MPS-based metrics, while fits using the $50^{\text {th }}$ percentile MPS were better than the $95^{\text {th }}$ for nearly every metric; e.g., $\mathrm{R}^{2}=0.941$ and 1-NRMSE $=0.756$ for UBrIC $(r=2, p 2 p)$. Compared to GHBMC, model performance was slightly better using SIMon-based metrics; $\mathrm{R}^{2}>0.65$ for all fits; however, the relative performance of the metrics was similar. Critical values for top performing metrics: UBrIC, $C_{i}$, and BrIC (refit) are provided in Table 5-2. Results based MPS $50^{\text {th }}$ percentile and CSDM are provided in Appendix $\mathrm{H}$.

Table 5-2: Critical values for top performing kinematic metrics determined from fits to strain-based metrics from GHBMC $(n=1595)$.

\begin{tabular}{cccccccccccc}
\hline Metric & & $\beta_{o}$ & $\omega_{x c r}$ & $\alpha_{x c r}$ & $\omega_{y c r}$ & $\alpha_{y c r}$ & $\omega_{z c r}$ & $\alpha_{z c r}$ & $\mathrm{R}^{2}$ & SSE & $\begin{array}{c}1- \\
\text { NRMSE }\end{array}$ \\
\hline $\begin{array}{c}\text { UBrIC (p2p) } \\
\text { (Equation 5-2) }\end{array}$ & MPS & 0 & 211 & 20.0 & 171 & 10.3 & 115 & 7.76 & 0.931 & 2.36 & 0.736 \\
\cline { 2 - 12 } & $\mathrm{CSDM}$ & -0.275 & 117 & 17.7 & 119 & 7.03 & 85.8 & 6.45 & 0.895 & 4.66 & 0.675 \\
\hline$C_{i}$ (peak) & $\mathrm{MPS}$ & 0 & 293 & 43.0 & 182 & 34.0 & 123 & 30.4 & 0.913 & 3.04 & 0.701 \\
\cline { 2 - 11 }$($ Equation 5-6) & $\mathrm{CSDM}$ & -0.327 & 181 & 28.6 & 96.7 & 31.9 & 80.7 & 25.8 & 0.892 & 4.80 & 0.671 \\
\hline $\begin{array}{c}\text { BrIC } \\
\text { (refit, peak) }\end{array}$ & $\mathrm{MPS}$ & 0 & 163 & - & 123 & - & 89.0 & - & 0.878 & 4.80 & 0.624 \\
\cline { 2 - 10 } (Equation 5-4) & $\mathrm{CSDM}$ & -0.358 & 99.9 & - & 71.5 & - & 58.9 & - & 0.847 & 6.78 & 0.609 \\
\hline
\end{tabular}

\subsubsection{Assessment by Impact Condition}

Top performing metrics include the peak-to-peak form of UBrIC and peak forms for $C_{i}$, and BrIC (refit), each with $r=2$. These three metrics were compared with the original BrIC using the impact conditions listed in Table 2-1. Relative to BrIC and BrIC (refit), UBrIC and $C_{i}$ performed better in the sled and crash test conditions; UBrIC was the top performing metric in nearly every mode (Figure 5-4, A and B). In particular, metricaccuracy in frontal (FRT), oblique (OBL), and side (SID) sled conditions were higher with UBrIC; 1-NRMSE > 0.90 for UBrIC in SID-SLED was the highest observed (Figure 5-4, 
B). Compared to the sled and crash test modes, performance in football impact conditions were more consistent among the metrics. While BrIC and BrIC (refit) performed better than UBrIC in several impact locations, $C_{i}$ tended to perform better in the majority of locations (Figure 5-4, C and D). Relative model performance based on CSDM and the $50^{\text {th }}$ percentile MPS were generally consistent with the $95^{\text {th }}$ percentile MPS (Appendix H).

(A) Correlations with automotive and sled impact conditions

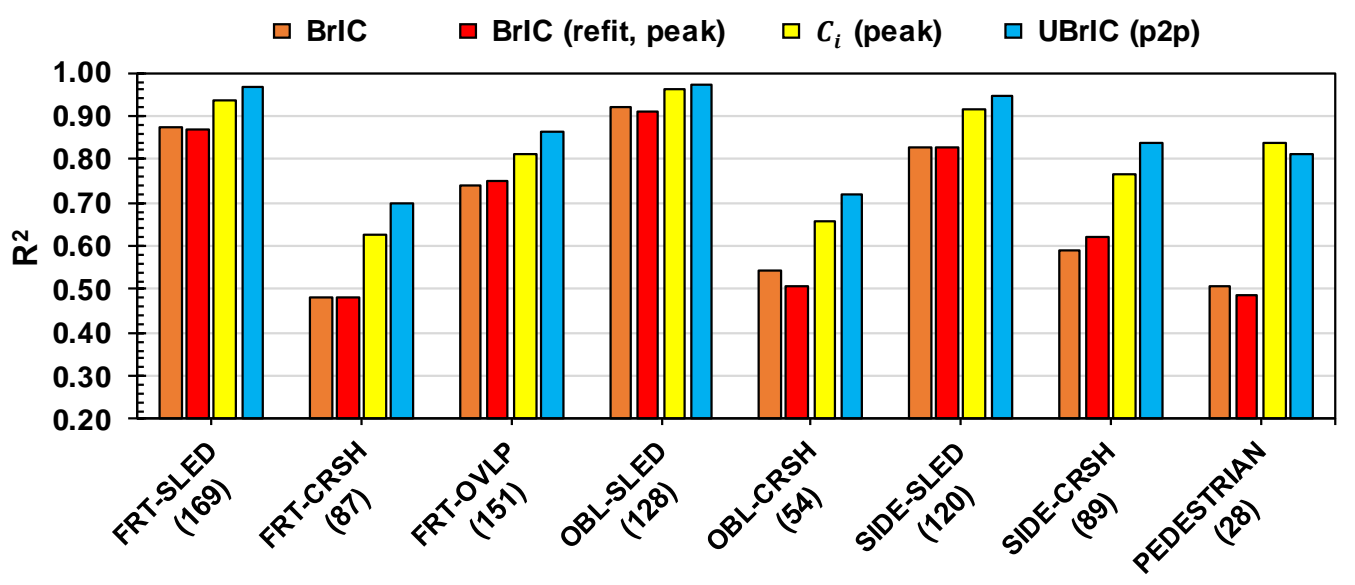

(B) Metric-accuracy with automotive and sled impact conditions

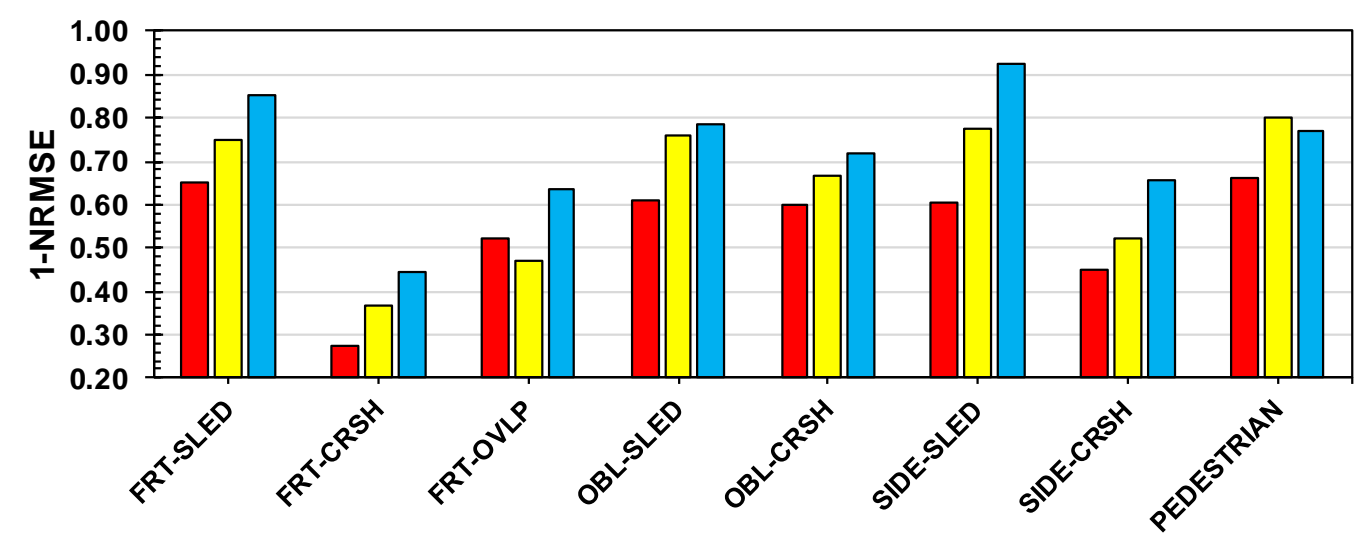

(C) Correlations with football impact conditions

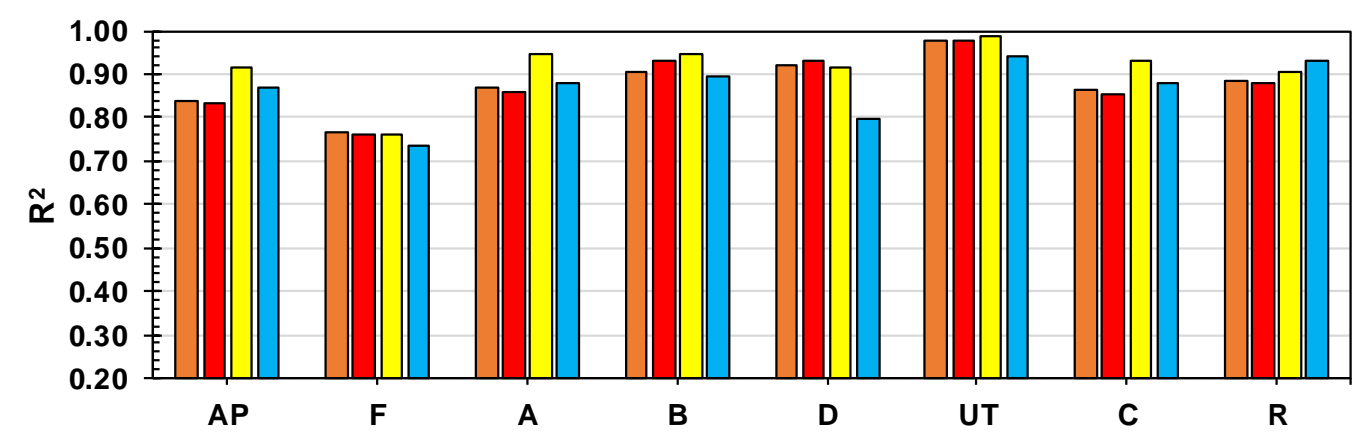


(D) Metric-accuracy with football impact conditions

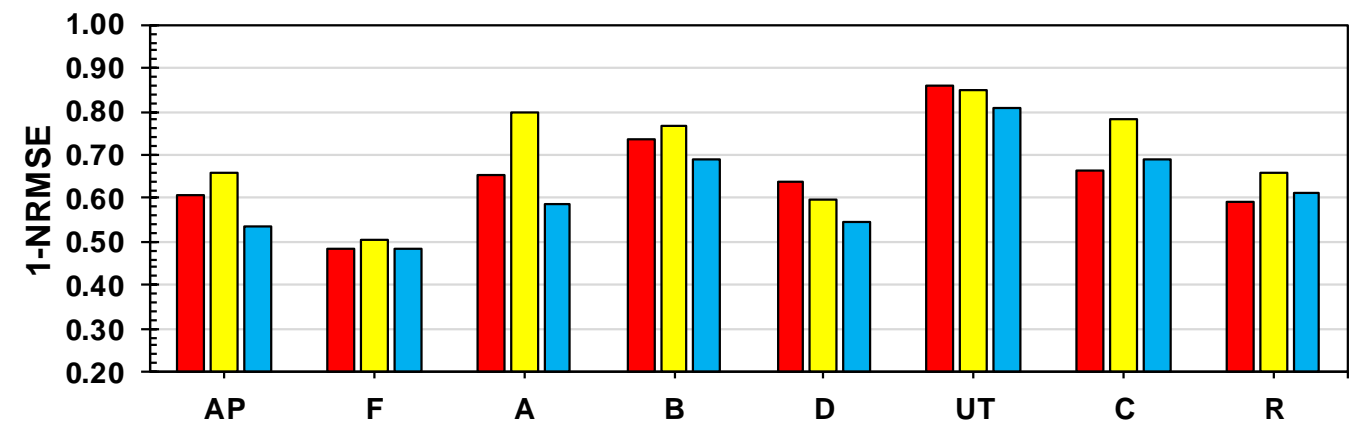

Figure 5-4: Performance of top performing fitted metrics and BrIC assessed by impact condition based on correlations $(A, C)$ and metric accuracy $(B, D)$ for automotive and sled $(\mathrm{A}, \mathrm{B})$, and football $(\mathrm{C}, \mathrm{D})$ impact conditions. Results are evaluated for metrics with $r=2$, and relative to MPS $\left(95^{\text {th }}\right.$ percentile) from GHBMC. Small and moderate overlap conditions (FRT-OVLP) were combined and assessed independently from the full frontal crash (FRT-CRSH) test mode. BrIC was not included in the metric-accuracy assessment because its values do not directly correspond to MPS and CSDM from GHBMC. Sample sizes for the sled and crash modes are shown in parenthesis $(A, B)$, there were 72 tests for each football impact locations $(C, D)$.

\subsubsection{Assessment using Idealized Rotational Head Motions}

When compared to $C_{i}$ and BrIC (refit), the relationship between UBrIC, angular velocity, and angular acceleration more closely matched the response patterns of the sDOF and FE models that were reported in Chapter 4 (Figure 5-5). The contours of BrIC and $C_{i}$ were vertical and diagonal, respectively, while the contours of UBrIC were more akin to the FE model; vertical for shorter duration pulses and horizontal for longer duration pulses with the transition occurring near the resonance frequency of the brain-skull system.

\subsubsection{Assessment using NHTSA Crash Tests}

Correlations and strain-based metric predictions were better using UBrIC with head impacts from the OMDB and frontal RD crash test modes (Figure 5-6). Using all 152 head impacts, correlations and metric accuracy were lower with BrIC and BrIC (refit) compared 
to $\mathrm{UBrIC}$ and $C_{i} ; \mathrm{R}^{2} \approx 0.6$ and $1-\mathrm{NRMSE} \leq 0.51$ for BrIC-based metrics, which was approximately 0.2 and 0.15 lower for $\mathrm{R}^{2}$ and 1-NRMSE, respectively than $\mathrm{UBrIC}$ and $C_{i}$.
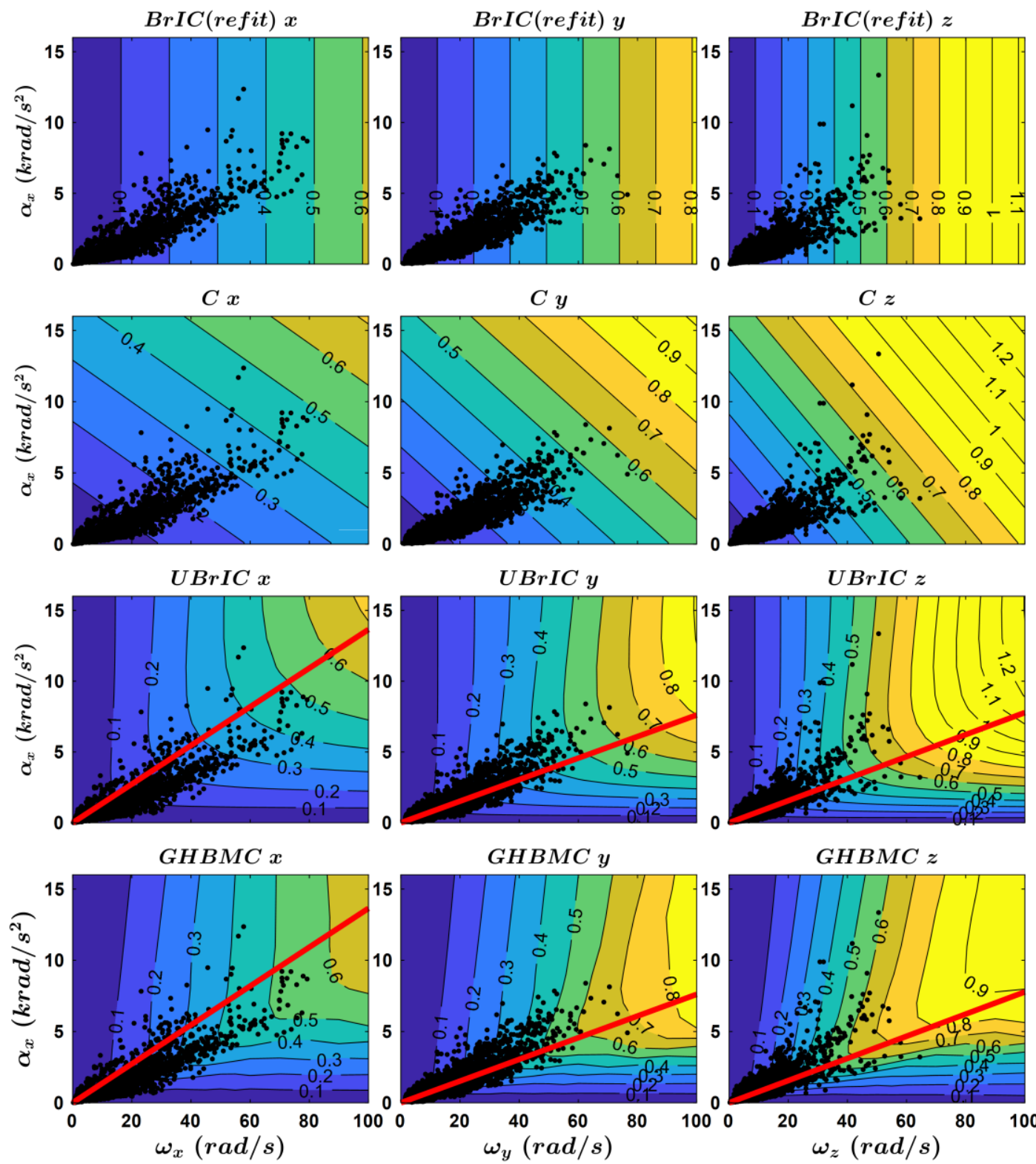

Figure 5-5: Comparison of MPS contours predicted by each of the top performing kinematic-based metrics (top 3 rows) relative to MPS from GHBMC (bottom row, reproduced from Figure 4-5. Contour lines represent constant MPS based on the peak angular velocity model forms. Black points are the peak angular velocity and angular acceleration about each axis from the 1595 head impacts. Solid red lines indicate the ratio of $\alpha_{i c r} / \omega_{i c r}$ which is inversely related to the effective one dimensional duration $\left(\Delta t_{i c r}\right)$; this line was obtained using the UBrIC peak model ( $3^{\text {rd }}$ row down) and overlaid onto the GHBMC MPS contours (bottom row). 


\subsection{Discussion}

Deformation is believed to be the primary mechanism for brain injury, and rotational head motion is the primary mechanism for brain deformation. However, existing kinematic-based metrics used in brain injury assessment do not represent brain strain over a broad range of head impacts. In this chapter, a new kinematic-based metric (UBrIC) was developed to predict strain-based responses (MPS and CSDM) from FE brain models using the directionally dependent magnitudes of angular velocity and acceleration from a head impact. UBrIC was formulated based on the governing relationship between maximum deformation and excitation from a second-order system, which was used as an analog for brain deformation to rotational head motion. The critical values of UBrIC were determined through fits to strain-based metrics (MPS and CSDM) obtained from FE simulation of 1595 head impacts in two different brain models. Efficacy of UBrIC for predicting strain-based response was assessed by comparing to fits using kinematic metrics with mathematical forms based on existing brain injury criteria. Comparisons were made through several assessments involving both real-world and idealized head motions.

\subsubsection{Comments on UBrIC}

Currently, UBrIC predicts MPS and CSDM obtained from the GHBMC and SIMon FE brain models, and has not been normalized to brain injury risk. Although brain injury criteria for MPS and CSDM were previously developed (Takhounts et al., 2013), these injury risk functions are not recommend to be used with UBrIC. The risk functions

developed by Takhounts et al., 2013 were fitted using the $100^{\text {th }}$ percentile MPS from SIMon. The current study uses the $95^{\text {th }}$ percentile MPS to avoid potentially spurious values generated using the maximum element. Furthermore, laboratory tests using human 
volunteers and field analyses involving crash reconstructions have shown that the MPSbased risk functions over-estimate brain injury risk (Laituri et al., 2015; Mueller et al., 2015; Sanchez et al., 2017). While the CSDM-based risk functions were more accurate when predicting non-injurious response, (Sanchez et al., 2017) their effectiveness at higher severity has not been validated. Until the existing strain-based risk functions can be fully verified using human injury data, or new risk functions developed using metrics from the current study, UBrIC should only be used for discriminating the relative severity between head impacts. This current limitation does not reduce the utility of UBrIC as it can still be used to inform design similar to how the GSI and HIC have driven improvements in helmet and automotive safety for decades (Gadd, 1966; Versace, 1971).

(A) Correlations with crash tests

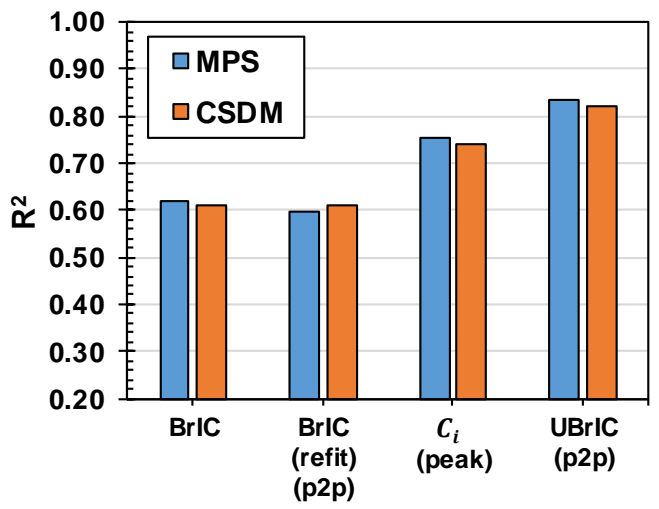

(B) Metric-accuracy with crash tests

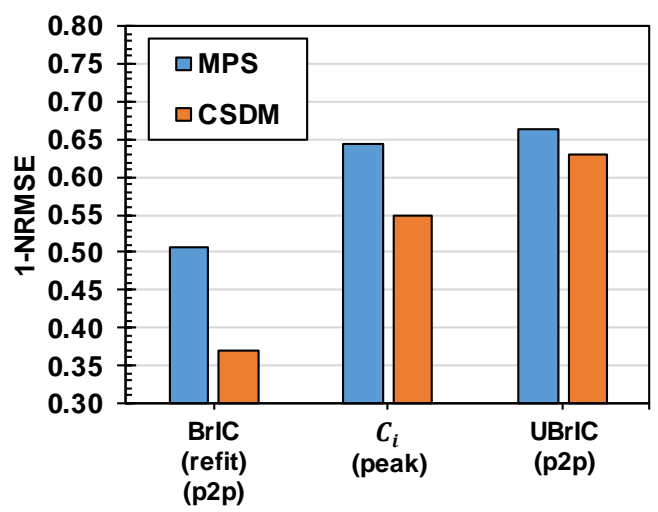

Figure 5-6: Performance of top performing fitted metrics and BrIC assessed for correlation (A) and metric accuracy (B) using NHTSA's OMDB and full frontal crash tests; 152 total head impacts. Results are evaluated for metrics with $r=2$, and relative to MPS $\left(95^{\text {th }}\right.$ percentile) from GHBMC.

Two separate approaches were used for evaluating the angular velocity magnitude of a head impact signal; peak and peak-to-peak. When evaluated relative to the peak model, the peak-to-peak version of UBrIC had a better fit to the overall database, and was more accurate in a majority of the impact conditions. Interestingly, the performance of BrIC 
(refit) and $C_{i}$ was generally worse using the peak-to-peak forms. Given that real-world impacts typically have more complicated time histories that can result in non-zero impact restitution, the peak-to-peak UBrIC model is recommended for predicting strain-based metrics. It is also recommended to use the UBrIC formulations with $r=2$, since overall model performance was slightly better than $r=1$, and to use the methods described in Chapter 2 for obtaining angular velocity and angular acceleration magnitudes. Use of parameters calculated in a different manner may substantially affect the prediction of strain-based metrics. Although direct measurement of head kinematics is preferred, this procedure has the advantage of obtaining angular accelerations without the high cost of deploying additional sensors in existing ATDs.

While exponential functions were used to represent the governing behavior of the second order system, mathematical forms based on other approaches were considered. Exponentials were chosen because of their decay and growth characteristics which allowed for a smooth and continuous transition between angular velocity and angular acceleration dependent brain deformations. Mathematical forms based on polynomials were also investigated; however, these models required additional critical values, which resulted in non-unique fits to achieve comparable performance to the exponential forms. Analytical solutions based on the magnitude of deformation from the sDOF model were also considered (Appendix F). While these formulations are derived directly from the solution to the EOM, their mathematical forms are cumbersome, and require a switching function to transition smoothly from short-to-long duration regimes. Furthermore, these solutions can only be obtained for a small set of idealized pulse shapes (unit step). Thus, their ability 
to accurately predict strain-based responses from FE models simulation of broad range of arbitrary impact pulses was limited compared to UBrIC.

\subsubsection{Comments on Existing Metric Forms}

In previous studies the rotational velocity change index (RVCI), a kinematic metric based on angular velocity change, performed well relative to existing rotational metrics, and exhibited similar response patterns to the mechanical models (Figure 4-10). Correlations between RVCI and strain-based responses from the current database were high; $\mathrm{R}^{2}=0.821$ and 0.776 for MPS and CSDM, respectively. However, calculation of RVCI is more complex compared to UBrIC, and involves maximizing the time history integral of angular acceleration in a manner similar to HIC. Pre-computed atlases have also been used for estimating FE model brain strain response to impact (Ji and Zhao, 2015). While this technique allows for whole brain strain computation, and is a cost-effective alternative to FE simulation, pre-computed values for real-world impacts are based on interpolations that are not based on brain deformation mechanics. Thus, the accuracy of this technique should be investigated for a broader range of head impacts involving longer duration and complex pulse shapes. Furthermore, a pre-computed atlas is a black-box function, which makes it difficult for engineers to understand how to manage the trade-offs between acceleration, velocity, and duration when designing a countermeasure.

While several kinematic metrics performed well with the overall database, UBrIC was a better predictor of strain-based responses in most impact conditions. Compared to other metrics, UBrIC performed better in nearly all of the automotive and sled conditions. This finding was anticipated, since impacts from these conditions were typically longer in duration; where brain deformation becomes more dependent on angular acceleration. 
However, improvement in the relative performance of UBrIC in football related impacts was not as marked. This finding was also anticipated, since direct head impacts typically result in shorter duration pulses where brain deformation response is proportional to angular velocity. While BrIC (refit) and $C_{i}$ performed well in these shorter duration conditions, the accuracy of UBrIC was generally better in the majority of impact conditions. Furthermore, the contours of UBrIC were more similar to the mechanical models when compared to the other metrics (Figure 5-5). Although BrIC (refit) and $C_{i}$ may be sufficient for predicting strain-based responses in some impact conditions, their applicability is limited to specific regimes of loading, i.e., BrIC can be used with shorter duration impacts, while $C_{i}$ can be used with moderate duration impacts; UBrIC can be used with impacts of all duration.

Relative to UBrIC, BrIC (refit) systematically over-estimated MPS and CSDM for low-to-moderate severity head impacts. These impacts were primarily from sled conditions, and included human volunteer and far side tests. When compared to the crash and impactor data, these cases typically had lower acceleration (higher duration), since direct head contact was either mild or did not occur. This finding suggests that the mathematical form of BrIC is insufficient for predicting strain-based responses for head impacts covering a broad range of durations, and thus angular acceleration should be included to improve prediction in higher velocity, lower acceleration (longer duration) events. By re-tuning the critical values of BrIC through fits to the current dataset the overall correlation with brain strains was improved; however, without angular acceleration, a metric based on angular velocity alone will lead to inaccurate strain predictions in certain head impact conditions. 
An additional concern with the use of metrics based only on angular velocity, is their potential insensitivity to improved safety countermeasures. For example, in several of the occupant and pedestrian crash tests used in the current study high levels of head angular velocity were achieved prior to head contact with a hard surface. By eliminating head contact through improvements made to safety countermeasures, one could reduce the magnitude of head angular acceleration without substantially changing the magnitude of angular velocity. Thus, a criterion based on angular velocity alone could be insensitive to an improved countermeasure design, and could potentially inhibit innovation. With current efforts focused on improving head safety systems, the changing landscape of countermeasure technology will likely test the limitations of existing metrics by potentially pushing their use into regimes where they are less accurate, and hence may not be able to affect injury countermeasures as is intended.

\subsubsection{Limitations with UBrIC}

The ability of UBrIC to predict brain injury relies heavily on the accuracy of the FE models. Although GHBMC and SIMon have been validated for brain deformation, the head kinematics and brain strain responses for some cases used in this study fall outside the range of experimental data used to validate these models (Chapter 3). Unfortunately, data for validating brain FE model is extremely limited to due challenges associated with human testing. Thus, future studies should focus on verifying the accuracy of FE model brain deformations over a broader range of head impacts. Furthermore, strain-based metrics used for fitting the critical values of UBrIC are based on global measures of maximum brain deformation. Strain rate, the product of strain and strain rate, fiber-oriented and region specific strain have also been proposed as brain injury predictors (Elkin and 
Morrison III, 2007; King et al., 2003; Sullivan et al., 2015). While these studies are encouraging, additional work is needed to determine whether incorporating these characteristics improves brain injury prediction in humans.

Brain injuries due to skull fracture or focal bleeding were not considered in the development of UBrIC. These injuries occur under high acceleration, short duration head impacts (Patrick et al., 1963), which may cause high localized strains within the head. In this dissertation, FE simulations were performed using a rigid skull to apply the 6DOF head kinematics. While several pedestrian tests involved human cadavers, none sustained a skull fracture despite achieving the highest recorded HIC values. Thus, skull deformations for head impacts included in the current study may be small enough to neglect.

\subsection{Conclusions}

A new kinematic-based brain injury metric, UBrIC, was developed based deformation response from a second order system, which was used as a mechanical analog for maximum brain deformation to rotational head motion. UBrIC uses the directionally dependent magnitudes of head angular velocity and angular acceleration to directly calculate strain-based responses (MPS and CSDM) from FE brain models. A total of 1595 head impacts covering a broad range of human response to impact were collected and simulated in two different FE models to obtain strain-based metrics for fitting the critical values. Relative to fits using kinematic metrics based on existing brain injury criteria, UBrIC was a better predictor strain-based responses in various head impact environments including those seen in automobile crashes and American football. Currently, UBrIC can only be used for assessing the relative severity between head impacts, since existing strainbased criteria have not been sufficiently verified using human injury datasets. 


\section{Chapter 6 - Multibody Model for the Human Brain}

Brain deformation due to rotational head motion is analogous to deformation from a second order mechanical system. Although kinematic-based metrics can quickly estimate maximum brain strain using peaks from the time history, their performance was found to be limited when used with more complicated head motions. This chapter presents the development of a multibody (MB) model for predicting brain deformation response to head impact. Three spring-mass-damper systems were used to estimate maximum brain strain (MPS) under three dimensional rotational head motion. Parameters for the MB model were determined using simplified pulses applied multiaxially to an FE brain model. System coupling was investigated, and strategies to reduce the overall number of parameters were considered in the formulation. Efficacy of the MB model for predicting FE model MPS was assessed using the head impact database.

\subsection{Introduction}

Multibody (MB) systems have been used extensively to model biomechanical responses to impact; from estimating skull and brain deformations during head impact (Stalnaker et al., 1971) to modeling lower limb forces during high rate axial loading (Henderson et al., 2013). These models consist of masses, springs, and dampers, which can be arranged to represent lumped body regions. The masses are rigid bodies which allow for application of forces and accelerations, while the springs and dashpots are massless connectors that transmit loads between the bodies. Simple MB systems have been used to model brain deformation occurring during head impact (Goldsmith, 1972). Typically, these models consist of one or two masses attached to a Kelvin-Voigt element with stiffness and damping properties based on the brain-skull system. The relative motion of the mass and 
base have been used as correlates for skull fracture, subdural hematoma, and diffuse brain injuries (Brinn and Staffeld, 1970; Fan, 1971; Slattenschek and Tauffkirchen, 1970; Stalnaker et al., 1971; Takahashi and Yanaoka, 2017).

Relative to FE models, MB models offer a reliable means of rapidly estimating brain-tissue deformations. Although MB models are coarser and provide less information about the local response, they are typically much quicker to use since they consist of far fewer equations to solve. In principle, MB models can be used to represent FE models over a broad range of head impacts, and allow for identification of prominent system properties including essential governing behaviors which may significantly reduce the complexity of the FE models required for predicting brain-strain (Laksari et al., 2015). MB models also have improved predictive capabilities over kinematic-based metrics, since whole time histories can be used as model inputs rather than a single point (peak). This advantage also helps to mitigate issues associated with complex waveforms including multiple impacts, which cannot be easily discriminated using kinematic-based metrics.

In chapter 5, UBrIC was shown to be the best kinematic-based predictor of strainbased metrics (MPS and CSDM) for a broad range of real-world head impacts. However, its formulation is based on the relationship between the magnitudes of an idealized pulse and maximum deformation from a one dimensional second order system. Real-world impacts often result in multiaxial head kinematics that consist of complex pulse shapes with multiple local peaks. Thus, a kinematic-based metric may not be adequate for predicting brain strains under these more complicated head motions. In chapter 4 , system parameters for three, uncoupled sDOF models were tuned using idealized, uniaxial head motions applied to a FE brain model so that the displacement output could be used to 
predict maximum brain strain response (Figure 4-8). Relative to existing kinematic-based metrics, deformation output from the three sDOF models was found to be the best correlate to MPS from the FE model using a subset of 660 real-world head impacts (Figure 4-9). While this preliminary MB model performed better overall, there were some impact conditions in which it over-predicted MPS relative to the kinematic-based metrics. One way to improve upon the existing MB framework is to include coupling terms, which would mitigate additional strains predicted by the uncoupled model.

This chapter presents the development of an MB model analogue for human brain deformation under rotational head motion. Coupling was incorporated into the framework of the MB model, and strategies to reduce the number of system parameters needed for solving the equations of motion was investigated. The proposed MB model will provide a better tool for estimating maximum FE brain strain over a broad range of head impacts.

\subsection{Methods}

\subsubsection{Multibody Model Development}

Given the similarity between the one dimensional deformation patterns of the sDOF and FE model (Figure 4-8), a MB model was constructed using three spring-mass-damper elements, which were used as analogues for maximum brain deformation due to rotational motion about each axis of the head (Figure 6-1). In matrix form, the EOMs for a damped, 3DOF coupled mechanical system under forced excitation at the masses are:

$[m] \ddot{\vec{\delta}}+[c] \dot{\vec{\delta}}+[k] \vec{\delta}=\vec{F}$,

Equation 6-1

where $[m]$ is a diagonal matrix of the system masses, $m_{i},[c]$, and $[k]$ are symmetric matrices that contain the damping, $c_{l m}$, and stiffness, $k_{l m}$ parameters $(l=m=1,2,3)$, and

$\ddot{\vec{\delta}}, \dot{\vec{\delta}}, \vec{\delta}$ and $\vec{F}$ are time history vectors of the accelerations, velocities, displacements, and 
applied forces for each mass, respectively. In expanded form, the mass, damping, and stiffness matrices are:

$$
\begin{aligned}
& {[m]=\left[\begin{array}{ccc}
m_{x} & 0 & 0 \\
0 & m_{y} & 0 \\
0 & 0 & m_{z}
\end{array}\right],} \\
& {[c]=\left[\begin{array}{ccc}
c_{11} & c_{12} & c_{13} \\
\cdots & c_{22} & c_{23} \\
\cdots & \cdots & c_{33}
\end{array}\right]=\left[\begin{array}{ccc}
c_{x}+c_{x y}+c_{z x} & -c_{x y} & -c_{z x} \\
\cdots & c_{x y}+c_{y}+c_{y z} & -c_{y z} \\
\cdots & \cdots & c_{z x}+c_{y z}+c_{z}
\end{array}\right],}
\end{aligned}
$$

Equation 6-3

and

$$
[k]=\left[\begin{array}{ccc}
k_{11} & k_{12} & k_{13} \\
\ldots & k_{22} & k_{23} \\
\ldots & \ldots & k_{33}
\end{array}\right]=\left[\begin{array}{ccc}
k_{x}+k_{x y}+k_{z x} & -k_{x y} & -k_{z x} \\
\ldots & k_{x y}+k_{y}+k_{y z} & -k_{y z} \\
\ldots & \ldots & k_{z x}+k_{y z}+k_{z}
\end{array}\right],
$$

Equation 6-4

respectively. The right hand side of Equation 6-1 was written in terms of the applied acceleration time histories, $\ddot{\vec{u}}=\left[\ddot{u}_{x} \ddot{u}_{y} \ddot{u}_{z}\right]^{T}$, where $T$ is the transpose of the tensor, and $\vec{F}=[m] \ddot{\vec{u}}$.

Equation 6-5

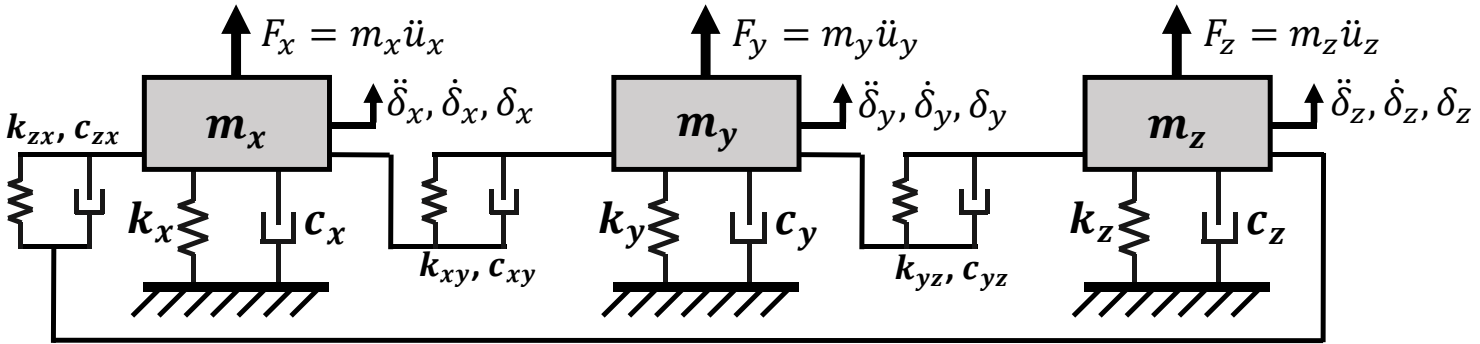

Figure 6-1:MB model analog for maximum brain deformation under multiaxial rotational head motion.

The solution to Equation 6-1, $\vec{\delta}=\left[\delta_{x}(t) \delta_{y}(t) \delta_{z}(t)\right]^{T}$, is a vector containing the displacement time histories of the three masses. These displacements are assumed to be 
analogous to brain deformation due to rotational motion about each axis of the head, and their magnitude is believed to be related to maximum brain strain (MPS). Two approaches were used to evaluate the magnitude of the MB displacements. The first assumes that MPS is related to the resultant of the maximum (res-max) displacement magnitude of each mass:

$M P S=\left\{\vec{\delta}_{m}^{T}[C] \vec{\delta}_{m}\right\}^{1 / 2}$

Equation 6-6

where $\vec{\delta}_{m}$ is a vector of the maximum displacements of the masses:

$\vec{\delta}_{m}=\left[\delta_{m_{x}} \delta_{m_{y}} \delta_{m_{z}}\right]^{T}$

Equation 6-7

and

$\delta_{m_{i}}=\max _{t}\left\{\delta_{i}(t)\right\}$

Equation 6-8

In this approach, the maximum displacements are allowed to occur at different points in the time history. The matrix $[C]$ is symmetric and contains scale factors that are used to relate the physical MB displacements to MPS:

$[C]=\left[\begin{array}{ccc}C_{x x} & C_{x y} & C_{z x} \\ \ldots & C_{y y} & C_{y z} \\ \ldots & \ldots & C_{z z}\end{array}\right]$,

Equation 6-9

The expanded form of Equation 6-6 can be expressed as

$M P S=$

$\sqrt{C_{x x} \delta_{m_{x}}{ }^{2}+C_{y y} \delta_{m_{y}}{ }^{2}+C_{z z} \delta_{m_{z}}{ }^{2}+2\left(C_{x y} \delta_{m_{x}} \delta_{m_{y}}+C_{y z} \delta_{m_{y}} \delta_{m_{z}}+C_{z x} \delta_{m_{z}} \delta_{m_{x}}\right)}$.

The diagonal terms, $C_{i i}$, in Equation 6-9 are related to the directionally dependent scale factors used with the three uncoupled sDOF models (Equation 4-7, Table 4-2). The offdiagonal terms, $C_{i j}$, for $i \neq j$, may also be used to account for system coupling. In this chapter, coupling will be investigated in two ways: first using Equations 6-3 and 6-4, where 
$c_{l m}, k_{l m} \neq 0$ for $l \neq m$, and $C_{x y}=C_{y z}=C_{z x}=0$, and second, where $c_{l m}, k_{l m}=0$ for $l$ $\neq m$, and $C_{x y}, C_{y z}, C_{z x} \neq 0$. The second approach to evaluating MB model output assumes that MPS is related to the maximum of the resultant (max-res) displacement time history: $M P S=\beta \max _{t}\{|\vec{\delta}(t)|\}$

Equation 6-10

where $\beta$ is scale factor that relates the maximum resultant MB displacement to MPS.

Proportional damping was assumed to reduce the number of parameters needed to solve for the system displacements. Proportional damping is technique that is commonly used to reduce the complexity of MB systems when the source and magnitude of damping are not exactly known (Rao and Yap, 1995). For the current MB model formulation, the damping matrix was expressed as a linear combination of the mass and stiffness matrices: $[c]=a_{o}[m]+a_{1}[k]$,

Equation 6-11 where $a_{o}$ and $a_{1}$ are the mass and stiffness proportional damping constants, respectively. The applicability of proportional damping for brain deformation was assessed using the uniaxial data from Chapter 4 ; in addition to the combined case $\left(a_{o}, a_{1} \neq 0\right)$, both massonly $\left(a_{1}=0\right)$ and stiffness-only $\left(a_{o}=0\right)$ proportional damping were investigated. Further detail on the solution to MB model (Equation 6-1) is provided in Appendix G.

\subsubsection{System Parameters Identification}

Several approaches were used to obtain values for the system parameters of the MB model. First, the values for the proportional damping constants were determined for the uncoupled model using the uniaxial data subsets described in Chapter 4. These subsets were originally used to obtain the directionally dependent natural periods, $\Delta t_{n_{i}}$, damping ratios, $\zeta_{i}$, and scale factors, $\beta_{i}$, for the three uncoupled, uniaxial models (Table 4-2). Since no proportional damping constraints were applied for the original optimizations, values for 
these parameters were refit for combined, mass-only, and stiffness-only proportional damping using the data subsets specified in Table 4-1. Model fits were performed separately for Equations 6-6 and 6-10 resulting in 8 total fits. The top performing dampingtype was chosen for each model-type as evaluated by either Equation 6-6 or Equation 6-10 based on the NRMSE metric (Equation 5-9). The resulting top two performing models were then used to investigated system coupling.

To investigate system coupling, a parametric study involving multidirectional, rotational-only pulses were used with the FE brain model. The magnitude, duration, and polarity of multiaxial angular velocity pulses were varied and applied to GHBMC. Pulse magnitudes were prescribed at six levels of isometric MPS (95th percentile element) for three different durations, $\Delta t_{i}$, based on the distribution of values from the database (Figure 6-2). Values for the angular velocity magnitudes, which were necessary to construct the time histories, were determined from the MPS contours (Figure 6-2) using target values for MPS and pulse duration (Table 6-1). An equal contribution to MPS was given to each direction; where the total expected MPS was set equal to the magnitude of the value from the three directions. In addition to these simulations angular velocity magnitudes were varied from $10-50 \mathrm{rad} / \mathrm{s}$ at $10 \mathrm{rad} / \mathrm{s}$ intervals for pulse durations of $(20,40$, and 60$) \mathrm{ms}$. These additional simulations were used to target coupled brain strains at equivalent inputs around system resonance. A total of 33 separate input conditions were used.

System coupling was assessed using the root sum square (RSS) of the uniaxial responses, i.e., the MPS resulting from a combined case (biaxial or triaxial) was compared with the RSS of the uniaxial responses resulting from the same input pulses:

$R S S_{i j k}=\sqrt{M P S_{i}+M P S_{j}+M P S_{k}}$.

Equation 6-12 
For example, $M P S_{x y}$ (the combined result from FE) was compared with $R S S_{x y}=$ $\sqrt{M P S_{x}+M P S_{y}}$, the RSS of the independent, uniaxial results (from FE). For a given pulse input set, if the result of Equation 6-12 was similar to the MPS from the combined case, then assumption of uncoupled response was valid, and could be captured via a RSS of the one-dimensional MB displacements, otherwise, the condition was considered to be coupled, and thus needed to be addressed through the coupling terms of the MB model.
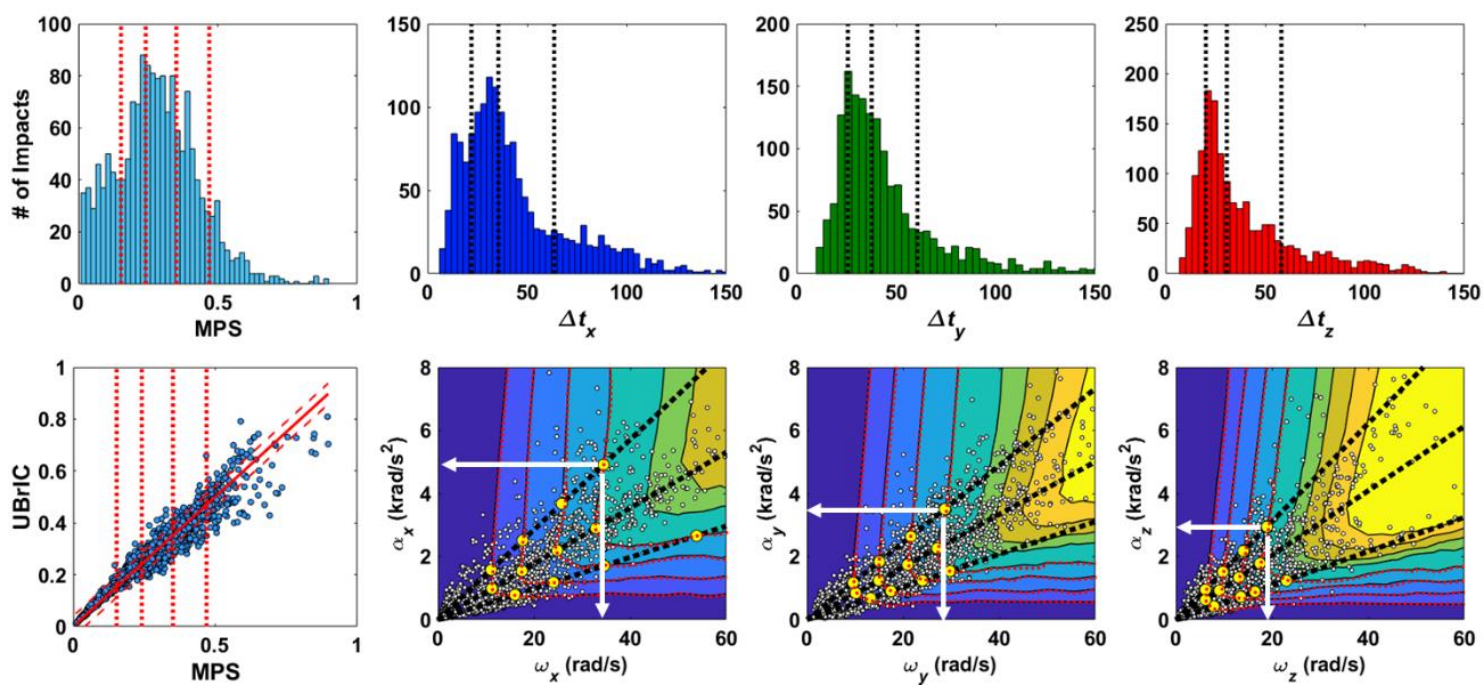

Figure 6-2: Method for choosing the pulse parameters used for the multiaxial parametric study. Red dotted lines on graphs in the first column represent the values of iso-MPS $\left(20,40,70\right.$, and $90^{\text {th }}$ MPS; Table 6-1, $1^{\text {st }}$ column, rows $\left.2-4\right)$, while black dotted lines indicate values of iso-duration $(20,50,90$ th percentile; Table 6-1, $3^{\text {rd }}-4^{\text {th }}$ column) that were chosen from the distributions for the MPS and directionally dependent durations of the head impact database (top row). Yellow points on the contour plots were used to construct the sinusoidal pulses. They show the location of the parametric simulations relative to the head impact database (small gray data points, $n=1747$ ).

Signal polarity (+/-) was also varied given that the brain is asymmetric about the sagittal plane. For rotations about a single axis, only $\pm y$ needed to be considered; however, results from Chapter 4 suggest that brain strain is insensitive to the direction of $y$ axis rotation (Figure 4-6). For biaxial and triaxial rotations there are 12 and 8 combinations, respectively; however only 6 for the biaxial case and 4 for the triaxial case are actually 
unique and need to be investigated (Table 6-2). FE simulations were performed for each of the 33 input conditions and for each unique head motion combination, resulting in a total of 462 simulations. A test matrix of the simulations is provided in Table 6-1. Only MPS responses from GHBMC were investigated in this chapter.

Table 6-1: Simulations performed for the multiaxial parametric study

\begin{tabular}{|c|c|c|c|c|c|c|c|c|}
\hline \multicolumn{2}{|c|}{ Iso-MPS (target) } & \multicolumn{3}{|c|}{$\Delta t_{i}(\mathrm{~ms})$} & \multicolumn{3}{|c|}{$\omega_{i}(\mathrm{rad} / \mathrm{s})$} & \multirow{2}{*}{$\begin{array}{c}\text { \# of } \\
\text { simulations }\end{array}$} \\
\hline$M P S$ & $M P S_{i}$ & $\Delta t_{x}$ & $\Delta t_{y}$ & $\Delta t_{z}$ & $\omega_{x}$ & $\omega_{y}$ & $\omega_{z}$ & \\
\hline \multirow{4}{*}{0.153} & \multirow{3}{*}{0.088} & 22.0 & 25.7 & 20.2 & 11.1 & 9.61 & 6.14 & 14 \\
\hline & & 35.5 & 37.7 & 30.7 & 11.2 & 10.1 & 6.14 & 14 \\
\hline & & 63.6 & 60.7 & 58.1 & 16.0 & 13.2 & 7.88 & 14 \\
\hline & \multirow{3}{*}{0.139} & 22.0 & 25.7 & 20.2 & 17.6 & 15.1 & 9.75 & 14 \\
\hline \multirow[t]{3}{*}{0.242} & & 35.5 & 37.7 & 30.7 & 17.5 & 15.0 & 9.08 & 14 \\
\hline & & 63.6 & 60.7 & 58.1 & 24.0 & 17.5 & 13.5 & 14 \\
\hline & \multirow{3}{*}{0.203} & 22.0 & 25.7 & 20.2 & 25.8 & 21.6 & 14.0 & 14 \\
\hline \multirow[t]{3}{*}{0.351} & & 35.5 & 37.7 & 30.7 & 24.8 & 21.0 & 13.1 & 14 \\
\hline & & 63.6 & 60.7 & 58.1 & 34.6 & 24.0 & 16.4 & 14 \\
\hline & \multirow{3}{*}{0.203} & 22.0 & 25.7 & 20.2 & 34.4 & 28.7 & 19.0 & 14 \\
\hline \multirow[t]{3}{*}{0.469} & & 35.5 & 37.7 & 30.7 & 32.8 & 27.1 & 17.5 & 14 \\
\hline & & 63.6 & 60.7 & 58.1 & 53.8 & 29.8 & 23.0 & 14 \\
\hline & & 22.0 & 25.7 & 20.2 & 38.6 & 32.0 & 21.2 & 14 \\
\hline \multirow[t]{3}{*}{0.526} & \multirow[t]{2}{*}{0.304} & 35.5 & 37.7 & 30.7 & 37.0 & 29.8 & 19.0 & 14 \\
\hline & & 63.6 & 60.7 & 58.1 & 63.1 & 34.2 & 24.7 & 14 \\
\hline & \multirow{3}{*}{0.350} & 22.0 & 25.7 & 20.2 & 44.8 & 36.5 & 24.6 & 14 \\
\hline \multirow[t]{3}{*}{0.606} & & 35.5 & 37.7 & 30.7 & 42.7 & 34.0 & 22.4 & 14 \\
\hline & & 63.6 & 60.7 & 58.1 & 74.5 & 38.5 & 28.2 & 14 \\
\hline & \multirow{3}{*}{-} & 20 & 20 & 20 & 10 & 10 & 10 & 14 \\
\hline \multirow[t]{3}{*}{-} & & 40 & 40 & 40 & 10 & 10 & 10 & 14 \\
\hline & & 60 & 60 & 60 & 10 & 10 & 10 & 14 \\
\hline & \multirow{3}{*}{-} & 20 & 20 & 20 & 20 & 20 & 20 & 14 \\
\hline \multirow[t]{3}{*}{-} & & 40 & 40 & 40 & 20 & 20 & 20 & 14 \\
\hline & & 60 & 60 & 60 & 20 & 20 & 20 & 14 \\
\hline & \multirow{4}{*}{-} & 20 & 20 & 20 & 30 & 30 & 30 & 14 \\
\hline \multirow[t]{3}{*}{-} & & 40 & 40 & 40 & 30 & 30 & 30 & 14 \\
\hline & & 60 & 60 & 60 & 30 & 30 & 30 & 14 \\
\hline & & 20 & 20 & 20 & 40 & 40 & 40 & 14 \\
\hline \multirow[t]{3}{*}{-} & \multirow[t]{3}{*}{-} & 40 & 40 & 40 & 40 & 40 & 40 & 14 \\
\hline & & 60 & 60 & 60 & 40 & 40 & 40 & 14 \\
\hline & & 20 & 20 & 20 & 50 & 50 & 50 & 14 \\
\hline \multirow[t]{2}{*}{-} & \multirow[t]{2}{*}{-} & 40 & 40 & 40 & 50 & 50 & 50 & 14 \\
\hline & & 60 & 60 & 60 & 50 & 50 & 50 & 14 \\
\hline
\end{tabular}

Target values for the MPS and $\Delta t_{i}$ for the first 18 rows were based on percentiles from the distributions from the database (Chapter 2). Pulse magnitudes were determined by prescribing an equal weighting to the different directions; $M P S_{i}=M P S / \sqrt{3}$. For each combination of pulse parameters (each row above), 14 simulations were performed (14 columns in Table 6-2). Pulse shapes were all sinusoidal (Figure 4-2, A). 
Time histories for each multiaxial head motion were constructed using Equations 4-4 through 4-6 (Figure 4-2, A). An additional $50 \mathrm{~ms}$ of time, $t_{\text {ext }}$, was added to each pulse given that simulations from the uniaxial study had achieved their peaks prior to this time. Head motions were applied at time $t=0$ to the GHBMC model in the local head anatomical coordinate system and MPS was extracted from the simulation output and used for MB model parameter fitting.

Table 6-2: Descriptions of unique head motion combinations used for the multiaxial parametric study.

\begin{tabular}{ccccc|cccccc|cccc}
\hline Direction & \multicolumn{1}{c}{ Uniaxial } & \multicolumn{1}{c}{ Biaxial } \\
\hline$\omega_{x}$ & 1 & 0 & 0 & 0 & 1 & 1 & 1 & 1 & 0 & 0 & 1 & 1 & 1 & 1 \\
$\omega_{y}$ & 0 & 1 & 0 & -1 & 1 & -1 & 0 & 0 & 1 & -1 & 1 & -1 & 1 & -1 \\
$\omega_{z}$ & 0 & 0 & 1 & 0 & 0 & 0 & 1 & -1 & 1 & 1 & 1 & 1 & -1 & -1 \\
\hline
\end{tabular}

Numbers shown indicate the polarity of the applied pulse, where positive $=1$, negative $=-1$, and 0 indicates that the direction was not used, e.g., the first column indicates that only the $+x$ direction used.

Several steps were taken to identify the parameters for MB coupling. First, optimizations were performed by fixing the proportional damping constants and scale factors and allowing the off diagonal terms of the stiffness matrix to fit. Additional fits were then performed by allowing different combinations of the proportional damping constants, diagonal stiffness, and masses to vary in the optimization. Multiple starting points were chosen for the fitting parameters, which were normalized $(0-1)$ and scaled so that when adjusted, they had the same relative weighting in the fit. A nonlinear, leastsquares solver (lsqcurvefit; Matlab) was used for the fits such that the SSE between the GHBMC-measured and MB model-predicted MPS was minimized.

\subsubsection{Model Performance Assessment}

Performance of the fitted MB models was assessed using the head impact database ( $n=1747)$ and impact conditions listed in Table 2-1. A total of five fitted MB models were 
assessed: three models based on Equation 6-6; one without coupling, one with coupling implemented through the off-diagonal terms of the stiffness and damping matrices; $c_{l m}, k_{l m} \neq 0$ for $l \neq m$, and $C_{x y}=C_{y z}=C_{z x}=0$, and a third with coupling implemented through the matrix of scale factors; $c_{l m}, k_{l m}=0$ for $l \neq m$, and $C_{x y}, C_{y z}, C_{z x} \neq 0$. The fourth and fifth models were based on Equation 6-10; one without coupling, and one with coupling implemented through the off-diagonal terms of the stiffness and damping matrices. Performance was assessed relative to the uncoupled model from Chapter 4, and kinematic-based metrics (BrIC and UBrIC). Assessments were made with $\mathrm{R}^{2}$ and the NRMSE. MB model displacement time histories for the head impact database were calculated numerically (ode45; Matlab).

\subsection{Results}

\subsubsection{Proportional Damping}

Fits performed with the constraint of proportional damping were similar to those without. Using the formulation based on Equation 6-6 (res-max) and without coupling, fits to the uniaxial data were practically the same for the three different damping-types; 1 NRMSE $\approx 0.86$ for each case (Table 6-3). However, when using the formulation based on Equation 6-10 (max-res), mass-only proportional damping was lower than the stiffnessonly and combined cases, which were nearly identical (Table 6-3). Relative to the unconstrained case, the stiffness-only and combined cases were lower for both formulations; however, for formulations based Equation 6-6 this difference was negligible. Based on this assessment, stiffness-only proportional damping was selected for the coupling analysis as it balanced the trade-off between accuracy and the number of parameters required to solve (two less than the unconstrained case). 
Table 6-3: Results for the proportional damping fits to the uniaxial data.

\begin{tabular}{ccccccccc}
\hline Model & Chp. 4 & $\begin{array}{c}\text { Mass \& } \\
\text { stiff }\end{array}$ & $\begin{array}{c}\text { Mass- } \\
\text { only }\end{array}$ & $\begin{array}{c}\text { Stiff- } \\
\text { only }\end{array}$ & Chp. 4 & $\begin{array}{c}\text { Mass \& } \\
\text { stiff }\end{array}$ & $\begin{array}{c}\text { Mass- } \\
\text { only }\end{array}$ & $\begin{array}{c}\text { Stiff- } \\
\text { only }\end{array}$ \\
\hline$\Delta t_{n_{x}}(\mathrm{~ms})$ & 36.2 & 36.0 & 37.6 & 35.9 & 33.3 & 32.0 & 27.8 & 32.0 \\
$\Delta t_{n_{y}}(\mathrm{~ms})$ & 44.9 & 45.2 & 44.4 & 44.6 & 40.8 & 37.5 & 39.0 & 37.5 \\
$\Delta t_{n_{z}}(\mathrm{~ms})$ & 38.4 & 38.7 & 38.1 & 39.3 & 39.9 & 43.6 & 56.0 & 43.6 \\
$n_{o}$ & - & 59.5 & 144 & 0 & - & $5.00 \mathrm{e}-07$ & 224 & 0 \\
$a_{1}$ & - & $3.35 \mathrm{e}-03$ & 0 & $5.77 \mathrm{e}-03$ & - & $6.30 \mathrm{e}-03$ & 0 & $6.30 \mathrm{e}-03$ \\
\cline { 2 - 8 }$_{x}$ & 0.525 & 0.462 & 0.431 & 0.505 & 0.626 & 0.623 & 0.496 & 0.623 \\
$\zeta_{y}$ & 0.466 & 0.447 & 0.510 & 0.406 & 0.587 & 0.531 & 0.696 & 0.531 \\
$\zeta_{z}$ & 0.416 & 0.455 & 0.438 & 0.462 & 0.345 & 0.457 & 0.999 & 0.457 \\
$C_{x x}$ & 7.61 & 6.61 & 5.51 & 7.41 & - & - & - & - \\
$C_{y y}$ & 6.70 & 6.26 & 7.73 & 5.72 & - & - & - & - \\
$C_{z z}$ & 16.0 & 17.5 & 17.3 & 17.2 & - & - & - & - \\
$\beta(1 / \mathrm{m})$ & - & - & - & - & 3.42 & 3.60 & 4.04 & 3.60 \\
\cline { 2 - 9 } $1-\mathrm{NRMSE}$ & 0.864 & 0.859 & 0.856 & 0.857 & 0.831 & 0.804 & 0.700 & 0.805 \\
$\mathrm{R}^{2}$ & 0.986 & 0.985 & 0.984 & 0.984 & 0.975 & 0.965 & 0.911 & 0.965 \\
\hline
\end{tabular}

Fits are based on the uniaxial data (Table 4-2). Italicized damping ratios were calculated from proportional damping coefficients using Equation G-4. $C_{x y}=C_{y z}=C_{z x}=c_{l m}=$ $k_{l m}=0$ for all fits. Chapter 4 (Chp. 4) model is the case without proportional damping.

\subsubsection{FE Model Coupling}

Results from the multiaxial parametric study revealed a coupling behavior in the GHBMC model. When head motions were applied simultaneously about the $x$ and $z$ axes, the RSS of the uniaxial cases were greater than the combined result (Figure 6-3, C). This coupling was also found to be directionally dependent, i.e., MPS for the $+x$ and $-z$ combination was greater than the MPS from the $+x$ and $+z$ combination, and happened to be closer to the RSS of the independent results (Figure 6-3, D). When the other biaxial conditions were assessed ( $x y$ and $y z$ ) the RSS from the uniaxial conditions matched well with the combined result suggesting that the FE MPS could be captured via a simple RSS of the uniaxial responses, i.e., no coupling terms needed (Figure 6-3, A, B, E, and F). These behaviors were also seen in the triaxial combinations, where the RSS of the uniaxial 
responses were insensitive to the $y$ direction, but sensitive to changes about the $z$ axis. A summary of the loading conditions resulting in coupling are shown in Table 6-4.

Table 6-4: Coupling conditions for the GHBMC model

\begin{tabular}{cccccccccccc}
\hline $\begin{array}{c}\text { Direction } \\
\text { Uniaxial }\end{array}$ & \multicolumn{1}{c}{ Biaxial } \\
\hline$\omega_{x}$ & 1 & 1 & 1 & 1 & 0 & 0 & 1 & 1 & 1 & 1 \\
$\omega_{y}$ & 1 & -1 & 0 & 0 & 1 & -1 & 1 & -1 & 1 & -1 \\
$\omega_{z}$ & 0 & 0 & 1 & -1 & 1 & 1 & 1 & 1 & -1 & -1 \\
\hline Coupling & No & No & Yes & No & No & No & Yes & Yes & No & No \\
\hline $\begin{array}{c}\mathrm{R}^{2} \\
\left(\widehat{M P S}_{i j k}, M P S_{i j k}\right)\end{array}$ & 1.00 & 1.00 & 0.99 & 0.99 & 0.99 & 1.00 & 0.99 & 0.99 & 0.99 & 0.99 \\
\hline $\begin{array}{c}\text { Slope of } \\
\text { Regression }\end{array}$ & 1.05 & 1.05 & 1.19 & $1.08^{\mathrm{a}}$ & 1.05 & 1.05 & $1.21^{\mathrm{a}}$ & $1.21^{\mathrm{a}}$ & 1.14 & 1.15 \\
\hline
\end{tabular}

${ }^{a}$ Indicates the RSS of the uniaxial components over-estimates the combined response, i.e., coupling reduces the FE output relative to the RSS of the uniaxial responses (based on a paired t-test, $\mathrm{p}<0.05)$.
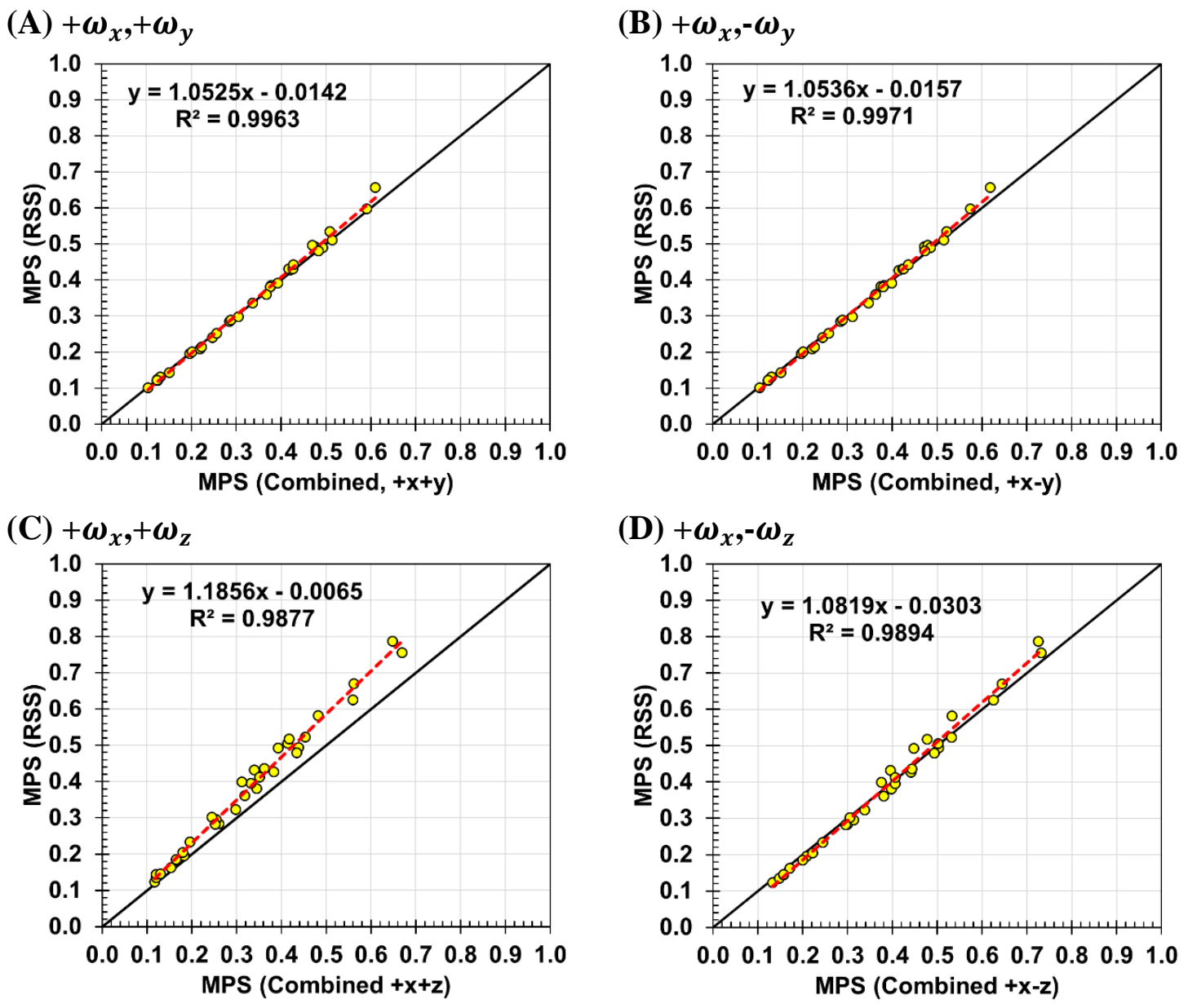

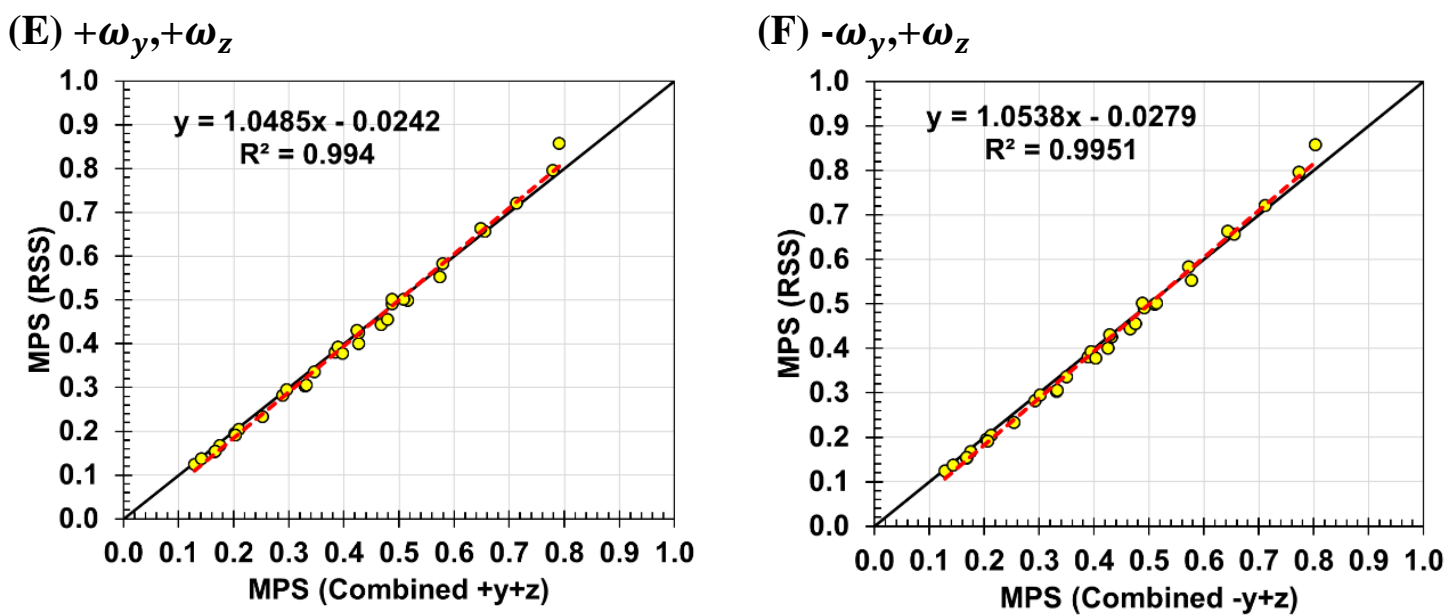

Figure 6-3: Comparison of FE model MPS from the biaxial loading cases (A-F; horizontal axes) relative to the RSS of the uniaxial FE MPS outputs at equivalent kinematic input (Equation 6-12; vertical axes).

Two approaches were used to characterize the coupling behavior seen in GHBMC:

First, the off-diagonal components of the scalar matrix (Equation 6-9) were determined. This was accomplished by minimizing the SSE between the $R S S_{i j k}$ from the uniaxial FE outputs modified with a coupling scale factor, and $M P S_{i j k}$ from the combined FE output. The regression included only the positive biaxial (3) and triaxial (1) directional loading cases $(n=132)$ resulting in 4 equations with three unknowns $\left(C_{i j}{ }^{\prime}\right)$, which were fit simultaneously using a nonlinear least squares solver (Solver, MS Excel):

$$
\begin{array}{ll}
R S S_{x y}=\sqrt{M P S_{x}{ }^{2}+M P S_{y}{ }^{2}+C_{x y}{ }^{\prime} M P S_{x} M P S_{y}}, & \text { Equation } \\
R S S_{y z}=\sqrt{M P S_{y}{ }^{2}+M P S_{z}{ }^{2}+C_{y z}{ }^{\prime} M P S_{y} M P S_{z}} & \text { Equation } \\
R S S_{z x}=\sqrt{M P S_{z}{ }^{2}+M P S_{x}{ }^{2}+C_{z x}{ }^{\prime} M P S_{z} M P S_{x}}, & \text { Equation } \\
R S S_{x y z}=\sqrt{M P S_{x}{ }^{2}+M P S_{y}{ }^{2}+M P S_{z}{ }^{2}+C_{x y}{ }^{\prime} M P S_{x} M P S_{y}+C_{y z}{ }^{\prime} M P S_{y} M P S_{z}+C_{z x}{ }^{\prime} M P S_{z} M P S_{x}}
\end{array}
$$

Equation 6-16 
Fits based on the positive biaxial and triaxial directional combinations revealed good agreement between the coupled RSS using the uniaxial FE MPS responses (Equations 6-13-6-16) and the values directly from the combined output (Figure 6-4, A-C, E). When assessed using the other biaxial and triaxial loading combinations (not used for fitting), the coupled RSS tended to under-predict cases involving a $+x$ and $-z$ rotation (Figure $6-4$, D, F; however, the other conditions remained unaffected. Values for the coupling parameters determined through fits using Equations 6-13 - 6-16 are shown in Table 6-5. These values were used in Equations 6-6-6-9 with the uncoupled, stiffness-only proportional damping MB model (Table 6-3, $5^{\text {th }}$ column).

(A) $+\omega_{x},+\omega_{y}($ Equation 6-13)

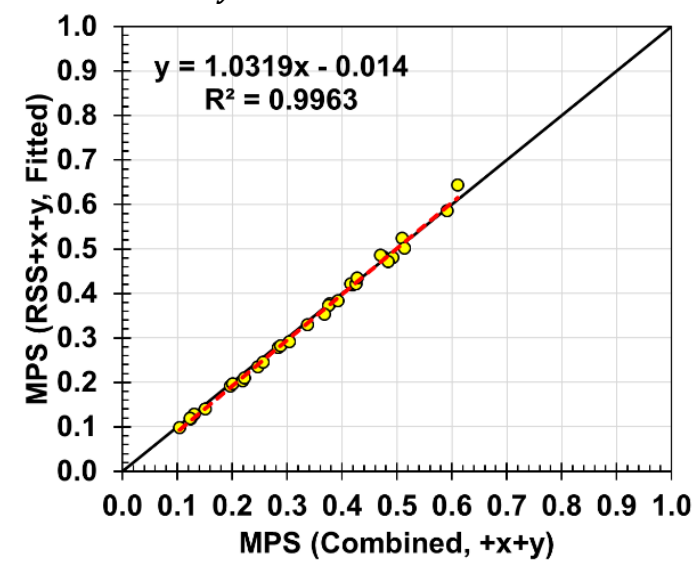

(C) $+\omega_{x},+\omega_{z}$ (Equation 6-15)

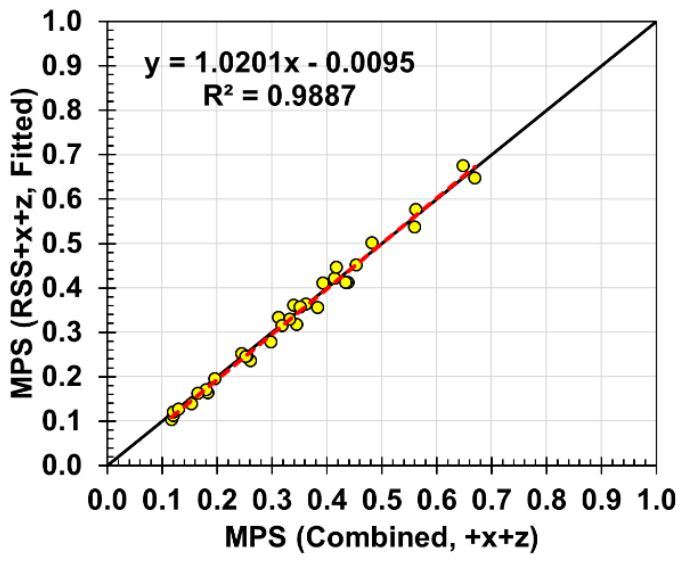

(B) $+\omega_{y},+\omega_{z}$ (Equation 6-14)

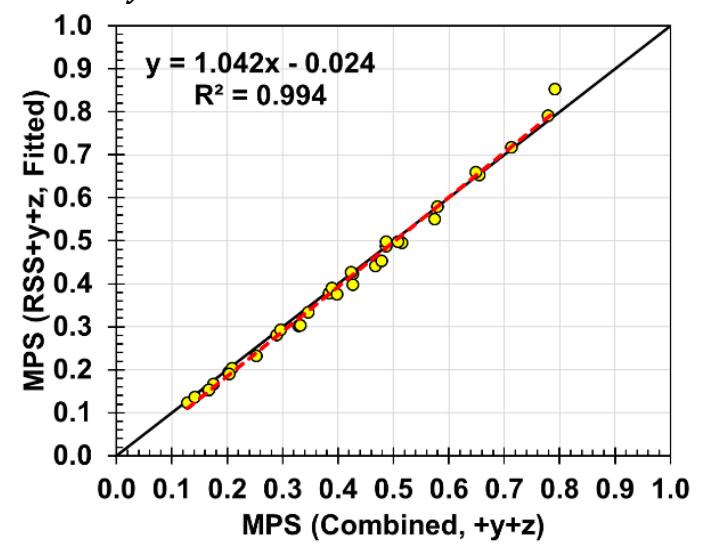

(D) $+\omega_{x},-\omega_{z}$ (Not included in fit)

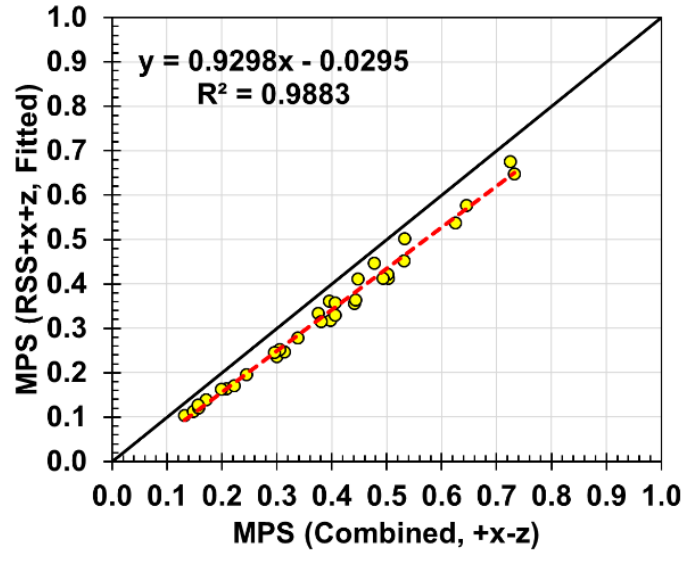


(E) $+\omega_{x},+\omega_{y},+\omega_{z}($ Equation 6-16)

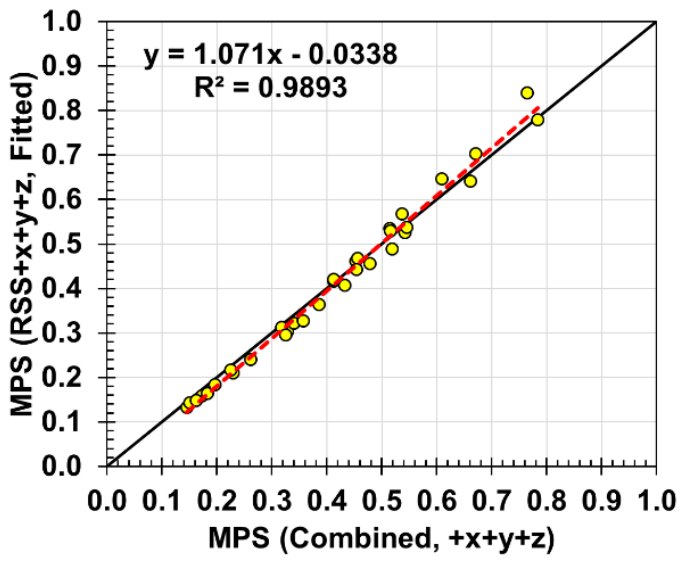

(F) $+\omega_{x},+\omega_{y},-\omega_{z}$ (Not included in fit)

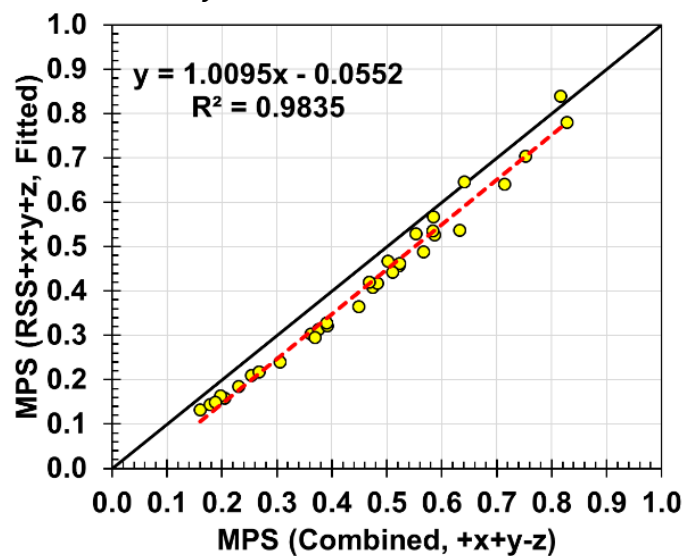

Figure 6-4: Comparison of FE model MPS from the biaxial loading cases (A-F; horizontal axes) relative to the fitted, coupled RSS of the positive uniaxial FE MPS outputs at equivalent kinematic input (Equations 6-13 - 6-16; vertical axes).

Table 6-5: Coupling coefficients from the regression analysis using GHBMC.

\begin{tabular}{cccc}
\hline$C_{x y}{ }^{\prime}$ & -0.080 & $C_{x y}$ & -0.259 \\
$C_{y z}{ }^{\prime}$ & -0.026 & $C_{y z}$ & -0.127 \\
$C_{z x}{ }^{\prime}$ & -0.605 & $C_{z x}$ & -3.41 \\
\hline
\end{tabular}

The scale factors for Equation 6-9 were determined by assuming $M P S_{i}=\beta_{i} \delta_{m_{i}}=$ $\sqrt{C_{i i}} \delta_{m_{i}}$ from Chapter 4 (Equation 4-7). Therefore, $C_{x y}=\frac{1}{2} C_{x y}{ }^{\prime} \beta_{x} \beta_{y}=\frac{1}{2} C_{x y}{ }^{\prime} \sqrt{C_{x x} C_{y y}}$ to satisfy Equation 6-9, which are based on the stiffness-only proportional damping values (Table $6-3,5^{\text {th }}$ column).

The second approach to characterizing the GHBMC's coupling behavior involved fitting the off-diagonal terms of the stiffness matrix. This was accomplished through multiple steps: First, optimizations were performed separately for each off-diagonal stiffness parameter by fitting only the parametric data with corresponding directions, e.g., only the biaxial $x$ and $\pm z$ data were used to fit $k_{13}=-k_{z x}(n=66)$. The value for the stiffness coupling term was then used as an initial guess to refit the corresponding diagonal components, e.g., $k_{11}$ and $k_{33}$ for $x z$ coupling, since the diagonal components of the stiffness matrix were originally fit without coupling parameters. Additional fits were performed by varying the other system parameters using the full parametric dataset $(n=$ 
429; excluding the $-y$ cases) to check the sensitivity of the optimized values. All fits using the off-diagonal components of the stiffness matrix were performed for Equation 6-6 with $C_{x y}=C_{y z}=C_{z x}=0$ and Equation 6-10.

To investigate the effect of directionally dependent coupling, which was observed only in the $x z$ direction, the force acting on $m_{z}$ was applied in the negative direction of motion. This was done to achieve the canceling effect on MPS seen in the parametric data, i.e., $+x$ and $+z$ direction excitation resulted in less strain in the FE model compared to $+x$ and $-Z$ excitation for equivalent kinematic input (Figure 6-3, C and D). Therefore, forcing the masses against each other for a $+x$ and $+z$ direction outcome would give less MPS relative to the $+x$ and $-z$ direction. Results for the coupled MB model fits to the multiaxial data, and the coefficients for the off-diagonal stiffness terms are included in Table 6-6.

\subsubsection{Performance Assessment using the Database}

Relative to the kinematic-based metrics, MPS predictions using the MB models were more accurate with the FE model output (Figure 6-5). When assessed using the full database $(n=1747), \mathrm{R}^{2}>0.95$ and $1-\mathrm{NRMSE}>0.73$ for the MB models, where $\mathrm{R}^{2}=0.93$ and 1-NRMSE $=0.72$ for $\operatorname{UBrIC}(\mathrm{p} 2 \mathrm{p})$, and $\mathrm{R}^{2}=0.84$ and $1-\mathrm{NRMSE}=0.60$ for $\mathrm{BrIC}$ (refit). Compared to the uncoupled MB models, the coupled models predicted MPS slightly better; 1-NRMSE > 0.77; where 1-NRMSE <0.76, for the uncoupled models. A summary of the coupled MB models developed in this chapter and their performance with the database is listed in Table 6-6.

The MB models also out-performed the kinematic-based metrics when assessed using the impact conditions listed Table 2-1 (Figure 6-6). Correlations and 1-NRMSE for the MB models were higher than the kinematic-based metrics for nearly every impact 
condition assessed. Relative to the uncoupled versions, the coupled MB models generally performed better in each impact condition. Although coupling reduced the accuracy in Helmet B for the max-res MB model, this loss in accuracy came with the improved prediction of MPS in D, UT, and Pedestrian conditions (Figure 6-7, B).

(A) $\mathbf{R}^{2}=0.84,1-\mathrm{NRMSE}=\mathbf{0 . 6 0}$

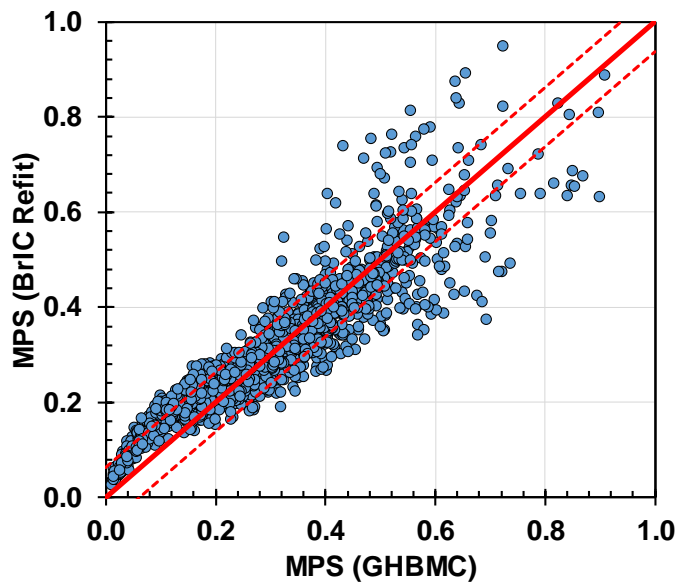

(C) $\mathbf{R}^{2}=0.96,1-\mathrm{NRMSE}=0.78$

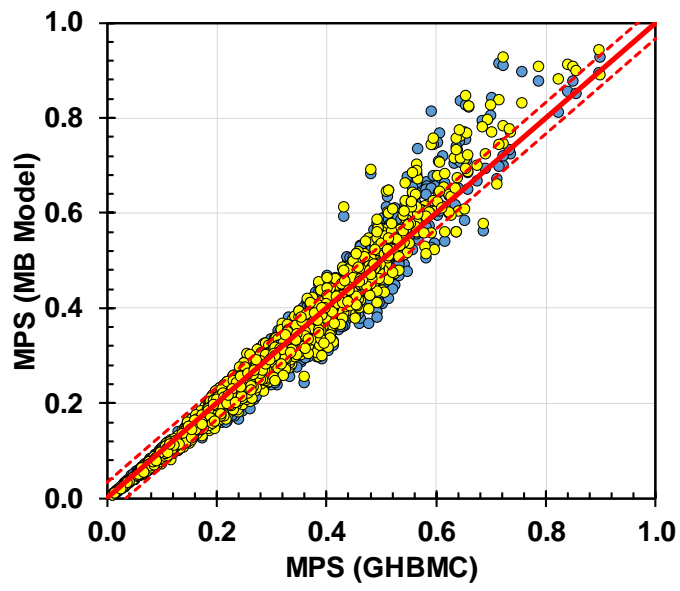

(B) $\mathbf{R}^{2}=0.93,1-\mathrm{NRMSE}=\mathbf{0 . 7 2}$

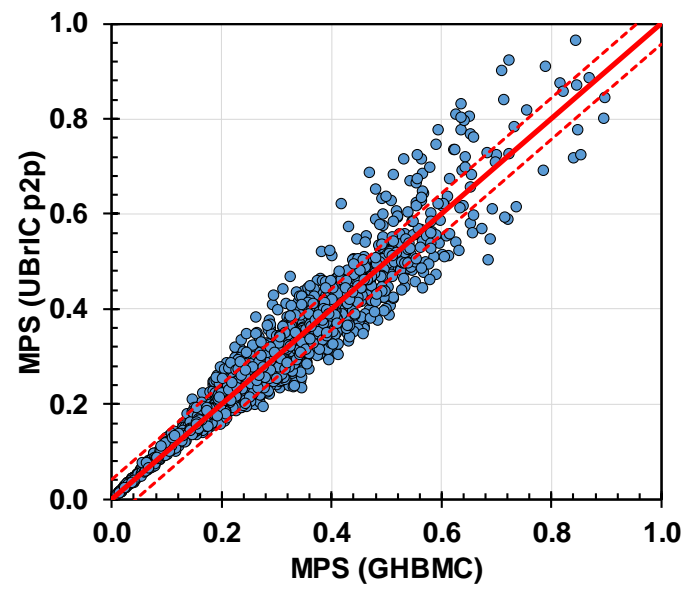

(D) $\mathbf{R}^{2}=0.97,1-\mathrm{NRMSE}=0.78$

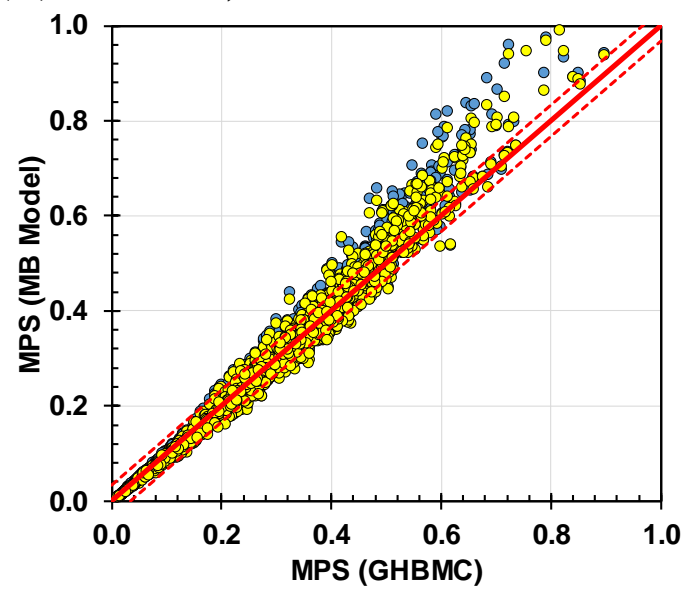

Figure 6-5: Scatter plots showing the prediction of MPS based on the metrics developed in this dissertation using the database $(n=1747)$. From $(A-D)$ shows improvement in fit with increasing model complexity, where kinematic-based metrics BrIC (A) and UBrIC (B) have three and six critical values, respectively and use only peaks from the time history. The stiffness proportional damping $M B$ models: two without coupling (blue points, $C$ and $D$ ) and two with coupling (yellow points, $C$ and $D$ ), based on the maximum of the time history resultant $(C)$, and resultant of the maximums from the time history (D). Values shown in the headers for $\mathrm{C}$ and $\mathrm{D}$ are for the coupled MB models only (yellow data points). 
Table 6-6: MB specifications and performance with the database $(n=1747)$.

\begin{tabular}{|c|c|c|c|c|c|}
\hline $\begin{array}{l}\text { Method of } \\
\text { calculation }\end{array}$ & $\begin{array}{l}\text { Equations } \\
6-6-6-9\end{array}$ & $\begin{array}{l}\text { Equations } \\
6-6-6-9\end{array}$ & $\begin{array}{c}\text { Equations } \\
6-6-6-9\end{array}$ & $\begin{array}{c}\text { Equations } \\
6-10\end{array}$ & $\begin{array}{l}\text { Equations } \\
6-10\end{array}$ \\
\hline Coupling & No & $\begin{array}{l}\text { Yes (matrix } \\
\text { of scalars) }\end{array}$ & $\begin{array}{c}\text { Yes (stiffness } \\
\text { matrix) }\end{array}$ & No & $\begin{array}{c}\text { Yes (stiffness } \\
\text { matrix) }\end{array}$ \\
\hline$m_{x}(\mathrm{~kg})$ & 1 & 1 & 1 & 1 & 1 \\
\hline$m_{y}(\mathrm{~kg})$ & 1 & 1 & 1 & 1 & 1 \\
\hline$m_{z}(\mathrm{~kg})$ & 1 & 1 & 1 & 1 & 1 \\
\hline$k_{x}(\mathrm{kN} / \mathrm{m})$ & 30.6 & 30.6 & 28.3 & 38.6 & 32.8 \\
\hline$k_{y}(\mathrm{kN} / \mathrm{m})$ & 19.8 & 19.8 & 19.8 & 28.1 & 23.6 \\
\hline$k_{z}(\mathrm{kN} / \mathrm{m})$ & 27.2 & 27.2 & 25.4 & 20.8 & 17.1 \\
\hline$k_{x y}(\mathrm{kN} / \mathrm{m})$ & 0 & 0 & 0 & 0 & 0 \\
\hline$k_{y z}(\mathrm{kN} / \mathrm{m})$ & 0 & 0 & 2.01 & 0 & 1.82 \\
\hline$k_{z x}(\mathrm{kN} / \mathrm{m})$ & 0 & 0 & 0 & 0 & 0 \\
\hline$\Delta t_{n_{x}}(\mathrm{~ms})$ & 35.9 & 35.9 & $35.5^{\mathrm{a}}$ & 32.0 & $33.7^{\mathrm{a}}$ \\
\hline$\Delta t_{n_{y}}(\mathrm{~ms})$ & 44.6 & 44.6 & $44.6^{\mathrm{a}}$ & 37.5 & $40.8^{\mathrm{a}}$ \\
\hline$\Delta t_{n_{z}}(\mathrm{~ms})$ & 39.3 & 39.3 & $38.7^{\mathrm{a}}$ & 43.6 & $46.0^{\mathrm{a}}$ \\
\hline$a_{o}(1 / \mathrm{s})$ & 0 & 0 & 0 & 0 & 0 \\
\hline$a_{1}(\mathrm{~s})$ & $5.77 \mathrm{e}-03$ & $5.77 \mathrm{e}-03$ & $5.77 e-03$ & $6.34 \mathrm{e}-03$ & $5.63 e-03$ \\
\hline$\zeta_{x}$ & 0.505 & 0.505 & $0.511^{\mathrm{a}}$ & 0.623 & $0.623^{\mathrm{a}}$ \\
\hline$\zeta_{y}$ & 0.406 & 0.406 & $0.406^{\mathrm{a}}$ & 0.531 & $0.531^{\mathrm{a}}$ \\
\hline$\zeta_{z}$ & 0.462 & 0.462 & $0.468^{\mathrm{a}}$ & 0.457 & $0.457^{\mathrm{a}}$ \\
\hline$C_{x x}\left(1 / \mathrm{m}^{2}\right)$ & 7.41 & 7.41 & 7.41 & - & - \\
\hline$C_{y y}\left(1 / \mathrm{m}^{2}\right)$ & 5.72 & 5.72 & 5.72 & - & - \\
\hline$C_{z z}\left(1 / \mathrm{m}^{2}\right)$ & 17.2 & 17.2 & 17.2 & - & - \\
\hline$C_{x y}\left(1 / \mathrm{m}^{2}\right)$ & 0 & -0.259 & 0 & - & - \\
\hline$C_{y z}\left(1 / \mathrm{m}^{2}\right)$ & 0 & -0.127 & 0 & - & - \\
\hline$C_{z x}\left(1 / \mathrm{m}^{2}\right)$ & 0 & -3.41 & 0 & - & - \\
\hline$\beta(1 / \mathrm{m})$ & - & - & - & 3.60 & 2.99 \\
\hline 1-NRMSE & 0.736 & 0.815 & 0.783 & 0.751 & 0.776 \\
\hline $\mathrm{R}^{2}$ & 0.968 & 0.975 & 0.972 & 0.954 & 0.965 \\
\hline \# of parameters & 10 & 13 & 11 & 8 & 9 \\
\hline
\end{tabular}

${ }^{a}$ Indicates the modal parameter (Appendix $\mathrm{G}$ ) that is associated with the local head coordinate direction. Gray italicized values indicate the quantity was either assumed or calculated based on the black text values, which were included in the optimization (excluding the masses).

\section{(A) Correlations with automotive and sled impact conditions}

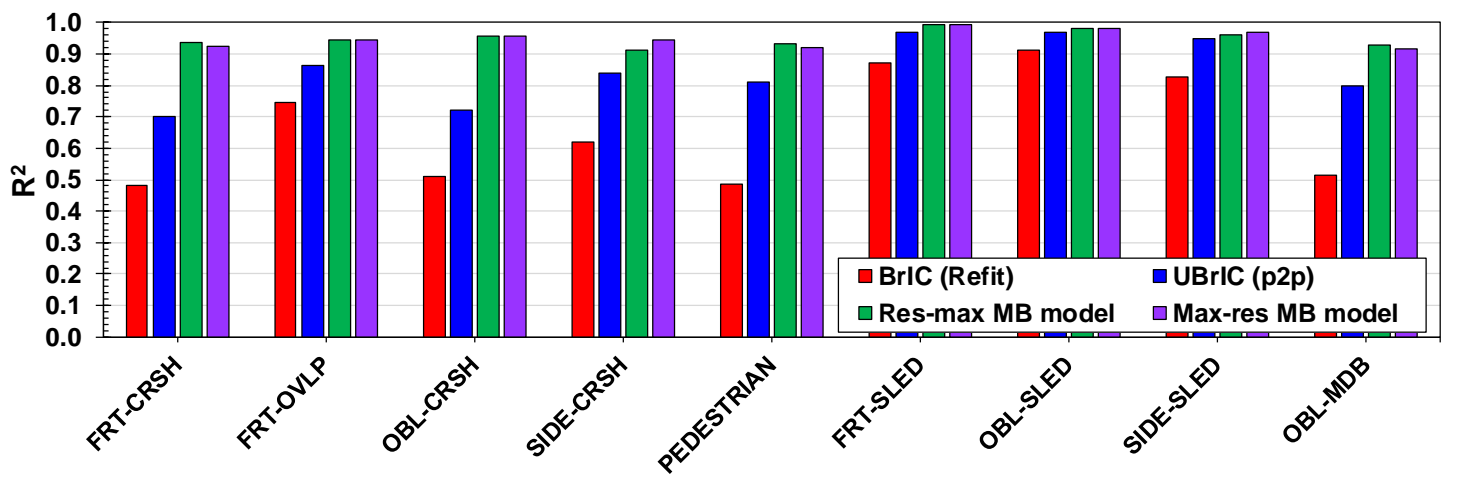


(B) Metric-accuracy with automotive and sled impact conditions

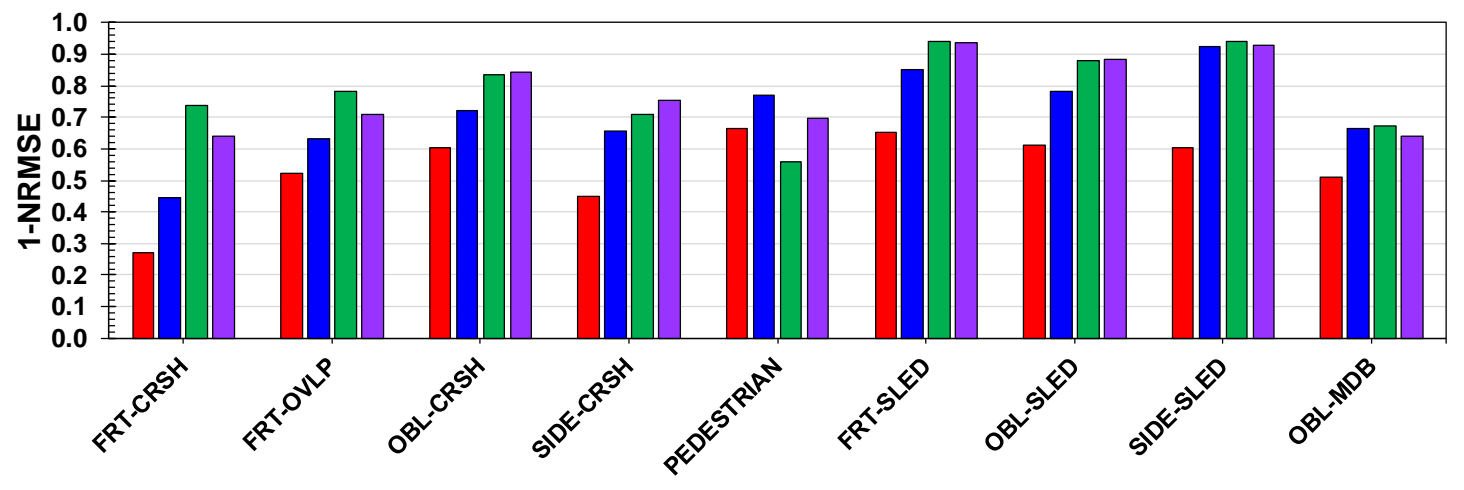

(C) Correlations with football impact conditions

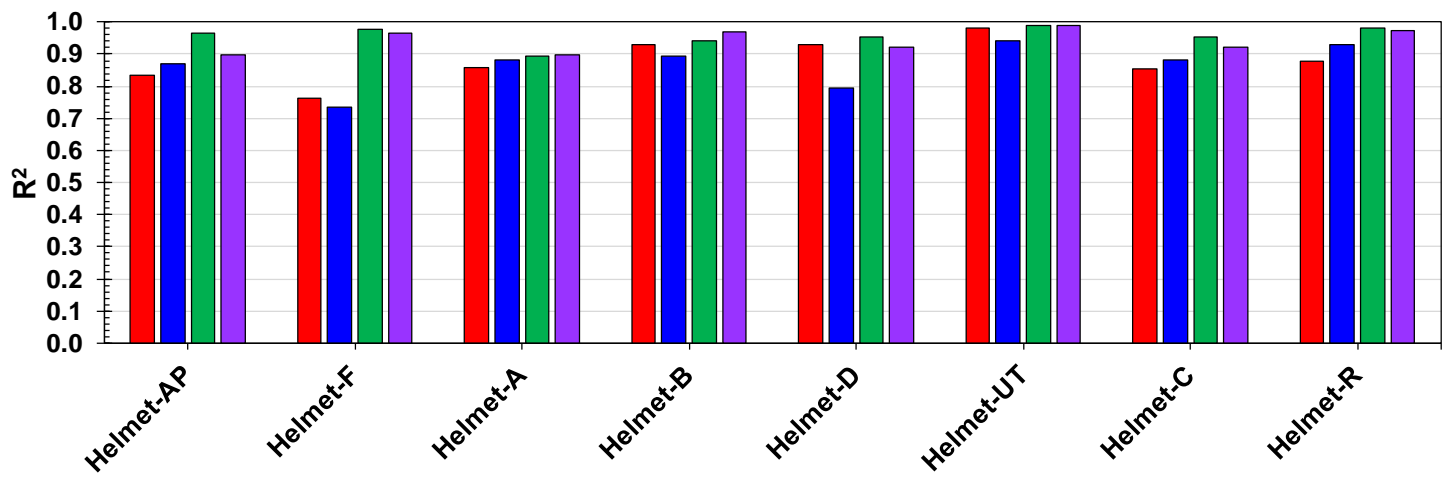

(D) Metric-accuracy with football impact conditions

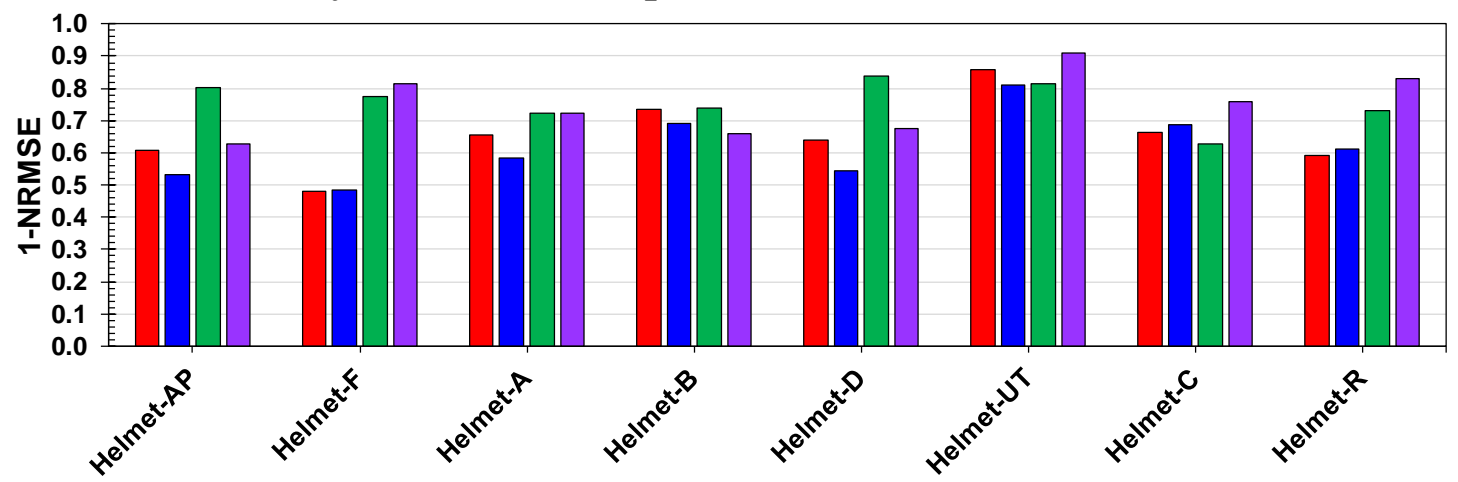

Figure 6-6: Performance of MB models and kinematic-based metrics assessed by impact condition based on correlations $(A, C)$ and metric accuracy $(B, D)$ for automotive and sled $(A, B)$, and football $(C, D)$ impact conditions. Results are based on predictions of MPS (95th percentile) from GHBMC. 
(A) Metric-accuracy with impact conditions (res-max MB models)

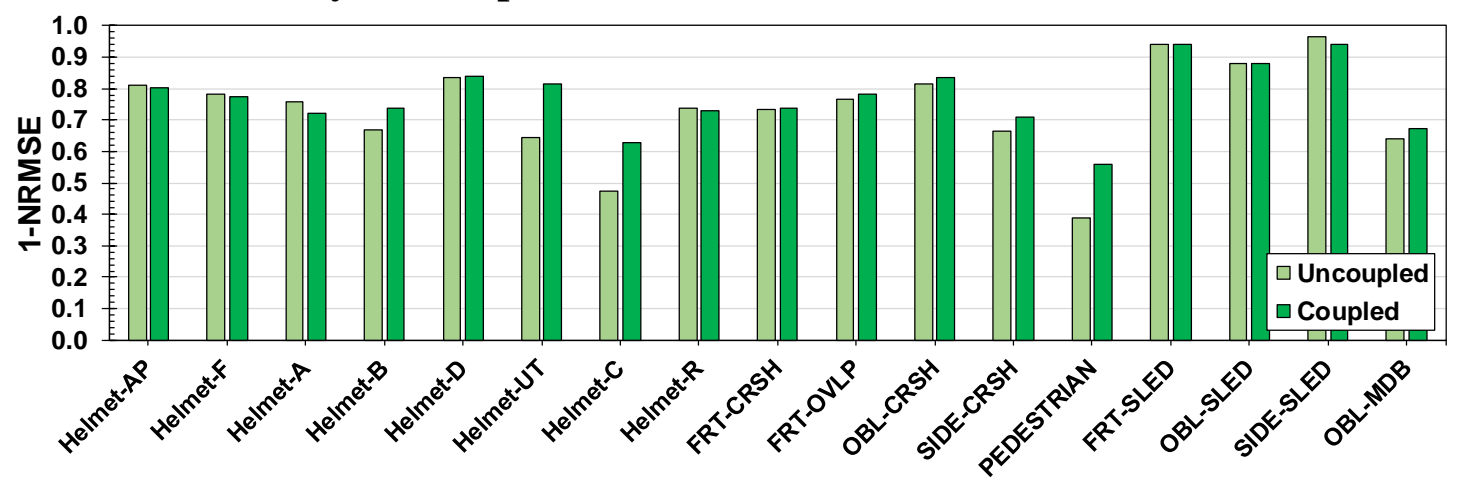

(B) Metric-accuracy with impact conditions (max-res MB models)

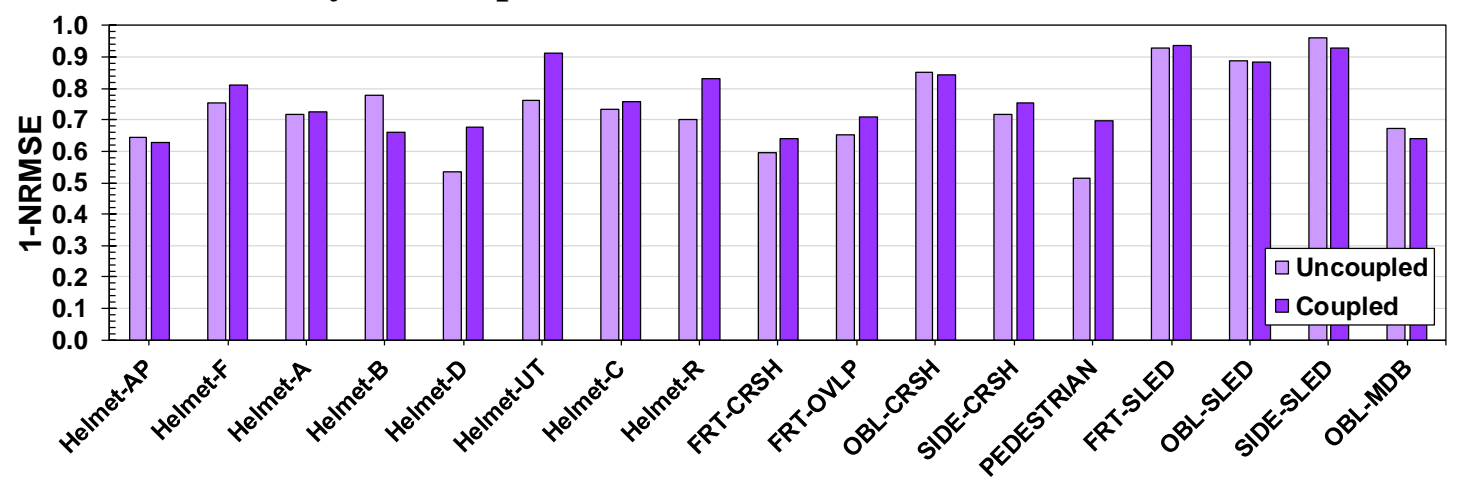

Figure 6-7: Comparison of uncoupled and coupled MB models assessed by impact condition based on metric accuracy for the res-max MB models (A) and max-res

MB models (B). Results are based on MPS (95th percentile) from GHBMC.

\subsection{Discussion}

Second order systems can be used to characterize brain deformations under rotational head motion. In this chapter, a MB model analog was developed to predict maximum brain strain from a head impact. Brain strains resulting from coupled head motions were investigated and incorporated into the model formulation, and strategies to reduce the number of parameters needed to solve the EOMs were implemented. System parameters for the MB model were obtained using a FE brain model and idealized rotational motions applied multiaxially to the head. Efficacy of the tuned MB models was assessed relative to kinematic-based metrics from Chapter 5, and a preliminary MB model 
developed in Chapter 4 using a database of more than 1700 head impacts from automotiveand football-related impact conditions.

\subsubsection{Applicability of the MB Model}

The MB models developed in this dissertation predict MPS ( $95^{\text {th }}$ percentile) from GHBMC. While brain strains from other FE models may be different, it is likely that a second-order system could be used to predict MPS from these models by adjusting the values of the system parameters. Although fits were not performed using CSDM, the correlation between $\mathrm{MB}$ model response and CSDM were higher relative to the kinematicbased metrics; $\mathrm{R}^{2}=0.9$. Thus, predictions of CSDM may be obtained using a linear regression of the MB model output. Similarly, correlations with the $50^{\text {th }}$ percentile MPS response were higher than kinematic-based metrics; $R^{2}=0.97$. This suggests that the $M B$ model parameters could be tuned to match other brain strain values by tuning the system parameters from the stiffness matrix.

Relative to models without proportional damping, MB models with proportional damping had similar performance with the database. This results suggests that damping in the human brain can be adequately captured by scaling the values of the brain's mass and stiffness. Although combined mass-and stiffness proportional damping had slightly better fit to the uniaxial data, their performance with the head impact database was practically the same. Given this similarity, the stiffness-only proportional damping model was chosen since it had one less parameter than the combined case.

Although the res-max MB model performed better than the max-res version, it's values are based on the maximum magnitudes of response from the time history, which can be taken at any point during the event. This approach is similar to how BrIC is 
evaluated, and may become problematic when used in conditions where multiple impacts occur over longer periods of time. This problem may be addressed by using the max-res MB model, which is evaluated based on the maximum response form the resultant time history displacement of the three masses. This technique has the capability to discriminate between brain deformations that arise from multiple impacts, since MB model output is evaluated over the time history of displacement, rather than peak deformations, which may be substantially out-of-phase (res-max). Although the max-res model had slightly lower performance with the overall database, it requires two less parameters to solve for the MPS prediction, therefore it is recommended for use over the res-max model.

\subsubsection{Comments on Coupling}

Of the unique head motion combinations considered, only those in which $x$ and $z$ were both positive resulted in a coupling effect that was over-estimated by the RSS of the uniaxial responses. This effect was also directionally dependent, as $+x$ and $-z$ combinations were correctly estimated by the RSS of the uniaxial cases, regardless of the polarity of the $y$ axis. When coupling was accounted for via the off-diagonal components of the scaling matrix, the overall performance of the coupled MB model improved. This improvement was at the expense of losing accuracy in some impact conditions, namely, Helmet B and $\mathrm{D}$, which were primarily $+x$ and $-z$ combinations; however, MB model to FE model MPS correlations remained similar, and better than kinematic-based metrics. When coupling was included in the stiffness matrix, only the $x z$ components were needed; coupling in the other directions were negligible based on fits to the multiaxial data. Although coupling terms improved the prediction of MPS, the res-max model still over-predicted at higher severity. This result may be due to construct of the current MB model, which assumes that coupling 
responses add linearly in the EOM (Equation 6-1). It is possible that a nonlinear $2^{\text {nd }}$ order system may be able to capture the nonlinear response observed in the FE model.

\subsubsection{Limitations of the Current Study}

Limitations associated with the FE model are applicable to the MB model as its response is based on FE output. Therefore, as the biofidelity of the FE model is improved, the MB model parameters can be re-tuned. Furthermore, the MB models predict the values of MPS, and not injury risk. Given the lack of reliable human brain injury datasets, it is recommended that the current $\mathrm{MB}$ metrics be used in a similar manner as UBrIC, i.e., for assessing the relative severity of head impact until existing risk functions are properly validated or improved using human injury datasets (Sanchez et al., 2017).

Other aspects contributing to variation in brain deformation response not addressed in the development of the MB models include age, sex, head shape/size. Additionally, the MB model predicts global MPS, which has previously been correlated with diffuse brain injury types (Takhounts et al., 2013). Evidence suggests that regional and axonal tractoriented strains may be better predictors of brain injury. Regardless, there is no evidence to suggest that a second-order system could not be tuned to target deformations caused by variations in anthropometry or for use with different strain-based metrics. Finally, strain rate has been suggested as a possible injury mechanism. Although strain rate was not considered in this dissertation, it is possible that the rate of deformation from the MB model could be tuned to match brain strain rates measured in the FE model during head impact.

\subsection{Conclusions}

In this chapter, a MB model was developed to predict MPS output from a FE brain model using the directionally dependent kinematic time histories of a head impact. System 
parameters were identified through several steps involving fits using idealized rotational motions applied uniaxially and multiaxially to the head. Fits using the uniaxial data revealed that the brain's structural damping could be adequately represented using stiffness proportional damping. Multiaxial head motions indicated a coupling response in maximum brain strain, which was apparent only in the combined coronal and axial head rotational cases $(x z)$. Based on these findings, both uncoupled and coupled MB models were fit to the idealized head motions. When verified using a database of more than 1700 real-world head impacts, the MB models were found to be better predictors of MPS relative to BrIC and UBrIC, emphasizing the advantage of using the time history of head kinematics with a second-order system in the prediction of brain strain. Given these results it is recommended that the MB models based on the maximum of the resultant time history displacement (max-res) response be used for estimating FE model MPS. This chapter provides an improved tool for estimating maximum brain strain during head impact, which can be used to discriminate the efficacy of improved helmets and countermeasures. 


\section{Chapter 7 - Conclusions}

\subsection{Major Contribution}

It is anticipated that the work from this dissertation will have a substantial impact on the assessment of brain injury. Most notable is the potential for the metrics developed in this dissertation to be used in crashworthiness and helmet safety evaluation. Given that BrIC has been proposed by NHTSA for brain injury risk assessment in crash tests (NHTSA, 2015), and that automotive safety research has traditionally influenced the development of head protective equipment and countermeasures in other fields (sport and military), BrIC is considered to be a leading brain injury risk assessment tool. Relative to BrIC, both UBrIC and the MB models developed in this dissertation have been shown to be better predictors of brain deformation across a broad range of automotive and helmet impact conditions, and thus have potential for becoming leading brain injury assessment metrics in these fields.

In particular, UBrIC addresses limitations with BrIC and other kinematic-based brain injury criteria by accounting for the duration of head impact in a manner that is consistent with fundamental mechanics. Relative to existing metrics, UBrIC is more effective at predicting strain-based metrics across a broad range head impact conditions, and may have the potential to discriminate the efficacy of improved countermeasures that may not otherwise be achievable using a brain injury criterion based on angular velocity alone (BrIC). This is important given that countermeasures tend to reduce the magnitude and increase the duration of head motion during impact. For example, it is easier to design a countermeasure that reduces the angular acceleration, since it is directly related to the force at impact whereas it can be more challenging to control the resulting motion of the 
head. Under these scenarios, UBrIC would be sensitive to changes in head kinematics because it includes angular acceleration, whereas BrIC may not be sensitive.

Compared to BrIC which uses only peak angular velocities, computation of UBrIC is relatively simple, and requires only three additional parameters from the impact time history; peak angular accelerations (Table 7-1). While the MB models out-perform both UBrIC and BrIC, their computation involves a numerical approach that requires the full head kinematic time histories (Table 7-1). Therefore, the MB models may be better employed as a research tool, possibly for projects that require large volumes of FE model simulations which may not be feasible with current computing resources. However, given that MB model computation times are still relatively low (compared to the FE model), their use in real-time assessments with wearable sensors and in crash testing is feasible.

Table 7-1. Summary of the metrics developed in this dissertation.

\begin{tabular}{cccc}
\hline Category & $\begin{array}{c}\text { MB model } \\
\text { (max-res) }\end{array}$ & UBrIC & $\begin{array}{c}\text { BrIC } \\
\text { (Refit) }\end{array}$ \\
\hline $\begin{array}{c}\text { Correlations with FE } \\
\text { MPS }(n=1747)\end{array}$ & 0.96 & 0.93 & 0.84 \\
$\#$ of model parameters & 9 & $6\left(\alpha_{i c r}, \omega_{i c r}\right)$ & $3\left(\omega_{i c r}\right)$ \\
$\#$ of equations to solve & 3 & 1 & 1 \\
Time histories required & Yes & No & No \\
Computational approach & Numerical & Analytical & Analytical \\
Compute time/impact & $1-3 \mathrm{sec}$ & $<1 \mathrm{sec}$ & $<1 \mathrm{sec}$ \\
\hline
\end{tabular}

\subsection{Other Contributions}

Other important contributions from this dissertation include the following:

1. The development of a database of head impact kinematics and corresponding brain strain responses (Chapter 2). The database developed in this dissertation includes 
more than 1700 head impacts, which consist of sled, crash, and impactor tests performed on various dummies, human volunteers, and cadavers. For each of these tests, 6DOF timed head kinematics are available and have been processed and prepared for FE model simulation. The database also includes head impact simulation results from two FE head models (GHBMC and SIMon). These results include the MPS strain time histories for every element in the brain, which were used to calculate strain based metrics (MPS and CSDM). These data may be leveraged in future studies for a broad range of applications including the development of brain injury risk functions, development region-specific TBI metrics, in addition to being used for crash and helmet safety evaluation.

2. The development of a turn-key method for rapid processing of head impact kinematics and extraction of FE brain strain responses. Matlab code was developed to automate the preparation of head kinematics from raw sensor measurements for $\mathrm{FE}$ simulation. The code involves a step-wise procedure that corrects and filters raw sensor measurements based on the methods described in Appendix A, and can quickly generate FE simulation files for a large volume of head impacts. The code can also process large volumes of FE simulation output quickly and store the results in a repository that can be accessed through user queries.

\section{Results from Chapter 3 indicate that brain injury criteria be based only on} rotational head kinematics. This conclusion is based on the finding that brain injury criteria using translation-only or combined kinematic metrics had poor correlation with brain strain metrics. This finding was not surprising given the nearly incompressible nature of brain tissue. Furthermore, when simulating the only the rotational components of head motions, brains strains were nearly identical to the combined (6DOF) motion, while simulating only 
the translational components resulted in negligible strain. This suggests that simulating only the rotational kinematics of a head impact is adequate for obtaining FE brain strains.

\section{Results from Chapter 4 show that brain deformation under rotational head} motion behaves as a second order mechanical system, and that metrics for brain injury criteria should include both angular velocity and angular acceleration. This conclusion is based on results from the parametric study using idealized uniaxial pulses applied to the FE and sDOF models. The contours of maximum brain strain from the FE model indicated a dependence on both angular velocity and angular acceleration, and were found to be remarkably similar to the maximum displacement contours from the sDOF model as well as the brain injury tolerances derived from animal studies. This dependence was categorized into three regimes of deformation response to head motion; a regime where deformation depended on velocity-only (short duration impacts), a regime where deformation depended on acceleration-only (long duration impacts), and a regime where deformation depended on both velocity and acceleration (resonance). Furthermore, when head impacts from the database were plotted on the contours of MPS, they spanned all three regimes. These results suggest that FE brain models can be adequately represented using a simple mechanical system, and that both angular velocity and angular acceleration are needed in brain injury criteria.

5. Results from Chapter 5 highlight the use of both angular velocity and angular acceleration for improved prediction of maximum brain strain. Using UBrIC improved prediction of brain strain across a broad range of head impact conditions when compared to BrIC, which is based on angular velocity-only. While UBrIC requires additional information from the time history (Table 7-1), it has the capability to improve prediction 
of brain strain in longer duration events. This is important for countermeasure design since they typically reduce the magnitude and increase the duration of impact; UBrIC is sensitive to these parameters.

6. Results from Chapter 6 highlight the use of a $M B$ model for rapid and accurate prediction of maximum brain strain response to head impact. The MB model improved prediction of brain strain across a broad range of head impact conditions when compared to kinematic-based metrics. Although the MB model requires the entire time history of a head impact and a numerical approach to solving, it provides a relatively quick estimation of maximum brain strain that is more accurate than the kinematic-based metrics. The MB model is also better suited for studying brain strain response in multiple impact scenarios, given that the recommended version is based on the resultant time history displacement.

\subsection{Future Work}

There are several areas in which the work from this dissertation can be expanded.

1. Development and validation of human brain injury riskfunctions. This is perhaps the most important task for the development of effective human brain injury criteria. The proposed risk functions should relate FE model brain strain responses (MPS and CSDM) to brain injury probability. This can be accomplished using both human and animal brain injury datasets. For example, quantified head impact kinematics and associated brain injury responses have been acquired from studies involving laboratory tests on pigs (Meaney et al., 1993) and sub-human primates (Abel et al., 1978; Ono et al., 1980; Stalnaker et al., 1977). This data could be used with a corresponding FE head model of the animal to establish tissue-level strain tolerances for humans (Antona-Makoshi, 2013). This approach assumes human and animal brain injuries occur at equivalent levels of strain, an assumption 
that should be verified with experimental data. Another approach to obtain tissue-level human tolerances involves either scaling the human FE model to the animal's anthropometry or scaling the animal's head kinematics to the human anthropometry (Takhounts et al., 2013). This approach assumes that brain injuries in the animals could be scaled to humans under equivalent head kinematics, and that the proportions of the brain and its substructures are similar between humans and animals; both are assumptions that some do not believe to be reliable (Ommaya et al., 1967). Regardless, methods for scaling animal data remains an open question for research (Sanchez et al., 2017).

Human datasets may also be used to develop and/or validate brain injury risk functions. Data from human volunteers provide sub-injurious responses, which have been used to verify efficacy of existing risk functions at lower severity (Sanchez et al., 2017). Head impact reconstructions of automobile crashes (Laituri et al., 2015; Mueller et al., 2015) or sports events (Hernandez et al., 2015) provide data for risk function development and validation at moderate-to-higher injury severity levels. Although the reliability of current head kinematic measurements and associated injury outcomes in sports is questionable (Sanchez et al., 2017), advancements in wearable sensors and video analysis offer solutions that may elucidate problems with existing methodologies. Human datasets also lack higher severity outcomes; however, this limitation may be overcome with the use of animal injury datasets that are appropriately adapted for use with human data. Given that diffuse brain injuries span a continuum of severities (Gennarelli et al., 1985; Margulies and Thibault, 1992), it is reasonable to assume that lower-to-moderate severity human injury datasets could be combined with higher severity animal injuries to develop an injury risk function that is applicable over a broad range of severities. 
2. Improvement of FE brain model biofidelity. FE model improvement and validation is an ongoing process. Results from this dissertation have already been used to guide the development of test methodologies for acquiring additional data for improving existing human FE head models (Alshareef et al., 2017). This dataset consists of human brain motion under rotational head kinematic loadings prescribed at magnitudes and durations relevant for automotive and football head impacts. These motions can be used to tune FE brain models for improved prediction of brain strain response during head impact. Future work may involve re-fitting the critical values of UBrIC and system parameters of the MB model to predict strain response (MPS and CSDM) from improved FE models. This could be accomplished using a randomly selected subset of the head impacts form the database for simulation in the improved FE model. If original and improved FE model responses are well correlated, then it may be possible to simply scale the values of UBrIC and the MB model. Another option may be to re-simulate the entire database, which could be accomplished in a reasonable amount of time given adequate computing resources.

\section{Development of brain injury criterion metrics based on other types of brain} strain. Only global MPS and CSDM were investigated in this dissertation. Evidence from the literature suggests that regional strains (Elkin et al., 2016; Hernandez et al., 2015), and axonal tract-oriented strains (Sullivan et al., 2015) may be better predictors of brain injury. Currently, human FE brain models have the capability to measure regional and tractoriented brain strains; however, there is little confidence in these measurements, since insufficient material property data exists to properly validate these aspects of the FE model. Thus, future work should focus on validating regional FE model responses and incorporating axonal tract information for simulation of fiber strain during head impact. 
Strain-rate and the product of strain and strain-rate have also been proposed as brain injury predictors. Clearly the rate of loading is important to the development of brain strain given the viscoelastic nature of the tissue; however, the role strain rate plays on human brain injury tolerance is not clear. Existing studies have proposed human tolerances based on FE simulation of head impacts obtained from laboratory reconstructions (King et al., 2003; Kleiven, 2007). Much skepticism exists in these reconstructions as they have not been scrutinized for biofidelity of their angular head motions, which is the primary cause of brain strain. Several experimental studies involving animals have shown brain injury tolerance to depend on strain rate (Cullen et al., 2007; Morrison et al., 2003); however, others studies have shown conflicting evidence (Bain and Meaney, 2000; Cater et al., 2006). Although strain rate may be an important parameter for predicting brain injury, additional work is necessary to clarify its effect on brain injury tolerance in humans prior to implementation in an injury criterion.

4. Applicability of metrics to different populations. The metrics developed in this dissertation predict brain strain response from a $50^{\text {th }}$ percentile, adult, human male. While there is no evidence to suggest that the metrics used in this dissertation could not be applied to the broader population, evidence suggests that brain strain depends on several aspects of anthropometry. Several studies have found brain material properties and structure to exhibit age dependency. While material property changes from infant to adult remains unclear (Prange and Margulies, 2002; Thibault and Margulies, 1998), there is more reliable evidence that suggests differences between adult and elderly human brains (Sack et al., 2009; Sato et al., 2003; Sowell et al., 2003). These include softer material properties, increased atrophy of the cerebral tissue, increased CSF volume, and decreased tissue failure 
tolerances with increased age. Injury tolerance has also shown to be dependent on sex, e.g., (Resch et al., 2017). For example emerging work suggests that there may be differences in tissue material properties between sexes, which would result in decreased brain strain tolerance for females (Finan et al., 2017). Furthermore, variation in head size, would result in different brain strains for the same material properties and internal structure of the brain. Regardless, no evidence suggests that a second order system could not be tuned to account for these anthropometric differences. For example, if brain strain tolerance to strain is increased, then the critical values of UBrIC could be increased, proportionally, and vice versa. Similarly, if the natural period of the system is increased, then ratio of the directionally dependent critical angular velocity to acceleration values could be increased. 


\section{Appendix A - Spatial Transformations}

This section provides the background and methods used for transforming raw head kinematic measurements from sensors to the local head anatomical coordinate system (SAE International). Sensor arrays include the Nine Accelerometer Package (NAP) and six-degree-of-freedom (6DOF) cube, which consist of three linear accelerometers and three Angular Rate Sensors (ARS). In this dissertation, the head is assumed to be a rigid body.

\section{A.1 Rigid Body Kinematics}

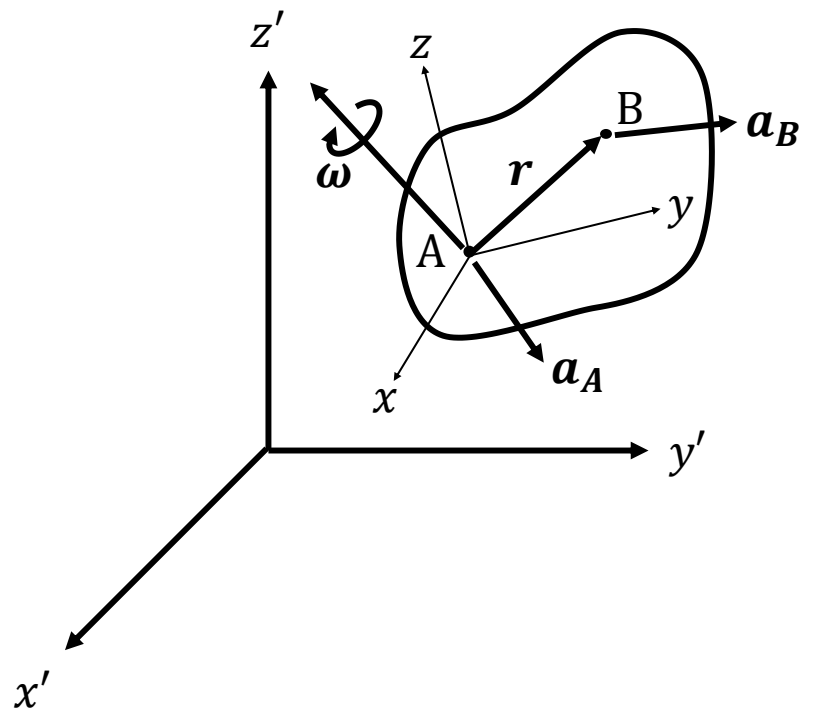

Figure A-1: Rigid body under arbitrary three dimensional motion.

Let $\mathrm{A}$ and $\mathrm{B}$ be two points on a rigid body in motion (Figure A-1). The equations of motion that describe the relationship between $\mathrm{A}$ and $\mathrm{B}$ are defined as

$a_{B}=a_{A}+\alpha \times r+\omega \times(\omega \times r)$,

Equation A-1

where $\boldsymbol{a}_{\boldsymbol{A}}=\left[\begin{array}{lll}a_{A, x} & a_{A, y} & a_{A, Z}\end{array}\right]^{T}$ and $\boldsymbol{a}_{\boldsymbol{B}}=\left[\begin{array}{lll}a_{B, x} & a_{B, y} & a_{B, Z}\end{array}\right]^{T}$ are the linear acceleration time history vectors of points A and B, respectively, $\boldsymbol{\omega}=\left[\omega_{x} \omega_{y} \omega_{z}\right]^{T}$ and $\boldsymbol{\alpha}=\left[\alpha_{x} \alpha_{y} \alpha_{z}\right]^{T}$ are the angular velocity and acceleration time histories of the body, respectively, and 
$\boldsymbol{r}=\left[\begin{array}{lll}r_{x} & r_{y} & r_{z}\end{array}\right]^{T}$ is the position vector that points from A to B. All measurements are referenced relative to a local (non-inertial) coordinate system $(x, y, z)$. The angular acceleration term in Equation A-1 arises from the acceleration of the non-inertial frame (tangential component) with respect to a global or fixed (inertial) frame $\left(x^{\prime}, y^{\prime}, z^{\prime}\right)$ while angular velocity term accounts from the centrifugal acceleration (normal component) (Thornton and Marion, 2004).

\section{A.2 NAP Kinematics}

NAP arrays consist of nine accelerometers that are rigidly mounted in a 3-2-2-2 configuration inside the head of a dummy with an origin fixed at the CG (Figure A-2). The advantage of this configuration over a 3-2-1 (six sensor) configuration is the added stability of three additional sensor measurements (Takhounts et al., 2009).

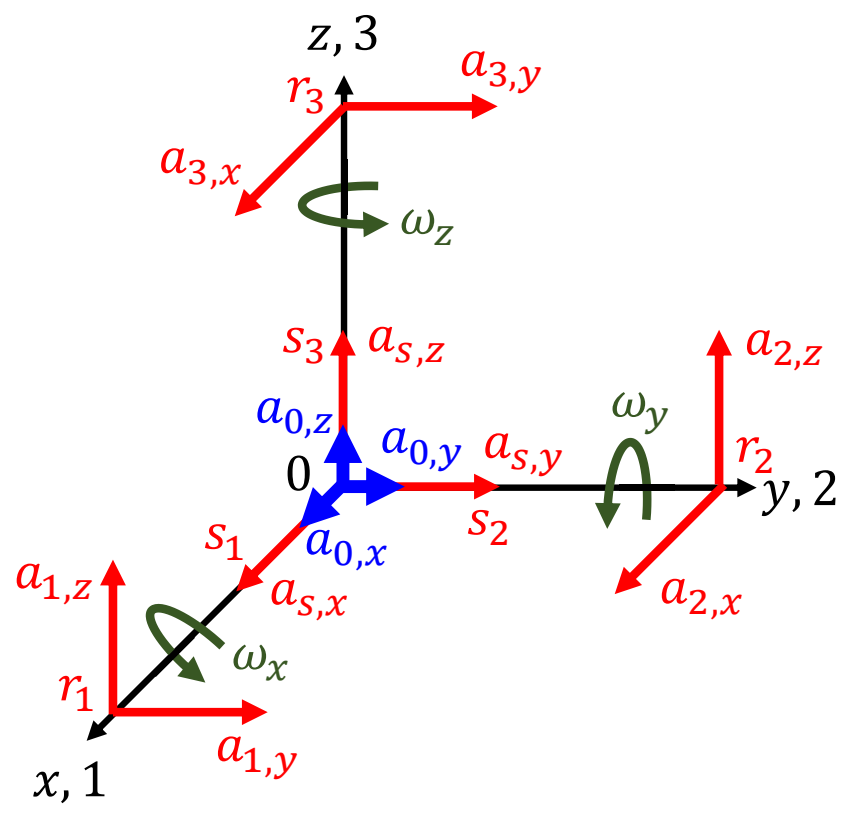

Figure A-2: NAP configuration (red arrows). Arm linear accelerometers are placed at locations $r_{1}, r_{2}, r_{3}$, while $\mathrm{CG}$ accelerometers are placed at $s_{1}, s_{2}, s_{3}$, and are assumed to be at the origin (0); each accelerometer measures linear acceleration at these points along the axes specified in red. 
To convert the NAP sensor measurements to 6DOF kinematics involves writing Equation A-1 to relate the origin accelerometer measurements $(0)$ to those at each arm (1, 2, and 3), separately (Padgaonkar et al., 1975). This results in a system of six equations which, when substituted and simplified, becomes three differential equations:

$$
\left\{\begin{array}{l}
\alpha_{x}=\frac{a_{2, z}-a_{0, z}}{2 r_{y}}-\frac{a_{3, y}-a_{0, y}}{2 r_{z}} \\
\alpha_{y}=\frac{a_{3, x}-a_{0, x}}{2 r_{z}}-\frac{a_{1, z}-a_{0, z}}{2 r_{x}} . \\
\alpha_{z}=\frac{a_{1, y}-a_{0, y}}{2 r_{x}}-\frac{a_{2, x}-a_{0, x}}{2 r_{y}}
\end{array}\right.
$$

Equation A-2

Equation A-2 defines the directionally dependent angular acceleration of the NAP array in the local NAP coordinate system. Typically, the NAP coordinate system is rotated $180^{\circ}$ about the $x$ axis relative to SAE-J211. In this case, the polarity of the $y$ and $z$ direction traces must be inverted prior to FE simulations. To obtain the angular velocity, $\boldsymbol{\omega}$, Equation A-2 must be integrated over the time history (trapezoid method):

$\omega_{i}\left(t_{j}\right)=\omega_{i}\left(t_{j-1}\right)+\frac{1}{2}\left(\alpha_{i}\left(t_{j}\right)+\alpha_{i}\left(t_{j-1}\right)\right)\left(t_{j}-t_{j-1}\right)$,

where $i$ is the anatomical direction $(x, y, z)$, and $j$ is the time point; $\omega_{i}\left(t_{0}\right)=0$. Integrating in the local frame assumes the time step of the motion to be relatively small, i.e., change in local coordinate system orientation relative to the fixed frame is small. Given that the sampling rate of the database was large $(10-20 \mathrm{kHz} \sim 0.1-0.05 \mathrm{~ms})$ relative to the motion of the event (10-300) ms, this error was considered to be negligible.

In 2009, Takhounts et al., proposed an algorithm that uses rigid body constraints to obtain a closed-form solution for each arm sensor measurement in terms of the eight other sensor measurements and arm lengths (Takhounts et al., 2009). This algorithm was implemented with the NAP tests from the head impact database as a check on the consistency of the rigid body head motion measured by the sensors. While several cases 
demonstrated inconsistent sensor measurements (based on visual inspection of the data), these tests were few $(n=12)$, and did not influence the development and assessment of the brain injury criterion metrics since the resulting 6DOF motion was behaved.

\section{A.3 6DOF Cube Kinematics:}

6DOF cubes were rigidly mounted either in the dummy's head at the CG or externally on the skull of a cadaver; Figure A-2 shows the 6DOF configuration where $\omega$ and $\boldsymbol{a}_{\boldsymbol{s}}$ are the corresponding 6DOF sensor measurements. Processing 6DOF head kinematics for the dummy is relatively straight forward and involves taking the derivate of the ARS measurements:

$\alpha_{i}\left(t_{j}\right)=\left(\omega_{i}\left(t_{j+1}\right)-\omega_{i}\left(t_{j-1}\right)\right) /\left(t_{j+1}-t_{j-1}\right)$.

Equation A-4

Equation A-4 describes a first order method (central difference), which was used on all interior points from the time history. A forward difference was used on the first time point and a backward difference on the last time point to avoid edge effects.

After calculating the rotational kinematics, linear accelerations were corrected for centrifugal motions since these sensors could not be physically placed at the origin of the cube, i.e., $\boldsymbol{a}_{\boldsymbol{o}}$ was calculated from $\boldsymbol{a}_{\boldsymbol{s}}$ (Figure A-2). The equations for correcting the linear acceleration measurements are derived from Equation A-1:

$$
\left\{\begin{array}{l}
a_{0, x}=a_{s, x}-s_{1}\left(\omega_{y}^{2}+\omega_{z}^{2}\right) \\
a_{0, y}=a_{s, y}-s_{2}\left(\omega_{z}^{2}+\omega_{x}^{2}\right) \\
a_{0, z}=a_{s, z}-s_{3}\left(\omega_{x}^{2}+\omega_{y}^{2}\right)
\end{array}\right.
$$

Equation A-5

This procedure was also implemented with NAP data; however, since rotational kinematics were calculated using accelerometer data that were not actually measured at the origin, correcting for non-coincident accelerations of a NAP array may lead to additional error. 
Fortunately, the sensor offsets, $s$, are generally small, and thus this correction is often neglected when processing head impact kinematics (Kang et al., 2011).

To process $6 \mathrm{DOF}$ head motions from sensor packages mounted externally to the skull of a cadaver consists of both rigid body and coordinate system transformations. The first step involves defining the transformation matrix between the externally mounted package (cube) and the fixed (global) coordinate systems:

$\boldsymbol{T}=\left[\begin{array}{cccc}\widehat{\boldsymbol{u}} & \widehat{\boldsymbol{v}} & \widehat{\boldsymbol{w}} & \boldsymbol{r}_{\boldsymbol{C}} \\ 0 & 0 & 0 & 1\end{array}\right]$

Equation A-6

where $\widehat{\boldsymbol{u}}, \widehat{\boldsymbol{v}}, \widehat{\boldsymbol{w}}$ are unit vectors, i.e., $\widehat{\boldsymbol{u}}=\left[\hat{u}_{1} \hat{u}_{2} \hat{u}_{3}\right]^{T}$, that describe the orientation of the cube in the global coordinate system, and are calculated using cube geometry. The vector $\boldsymbol{r}_{\boldsymbol{C}}$ is defined as the cube $(\boldsymbol{C})$ origin position vector in the global coordinate system. At this point, angular accelerations can be calculated from the cube ARS using Equation A-4, and the linear acceleration measurements in the cube can be corrected for centrifugal motions using Equation A-5; for convenience, cube sensors are typically mounted so their sensing axes intersect at the cube origin.

The next step involves defining the transformation matrix between the local (head) and global coordinate systems:

$\boldsymbol{K}=\left[\begin{array}{cccc}\widehat{\boldsymbol{x}} & \widehat{\boldsymbol{y}} & \hat{\boldsymbol{z}} & \boldsymbol{r}_{\boldsymbol{H}} \\ 0 & 0 & 0 & 1\end{array}\right]$

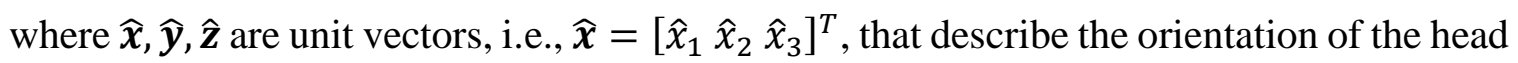
$(H)$ in the global coordinate system, and are calculated using anatomical landmarks on the skull (Robbins, 1983). The vector $\boldsymbol{r}_{\boldsymbol{H}}$ is defined as the head origin position vector in the global coordinate system. 
The final step involves constructing the transformation matrix between the head and cube coordinate systems, $\boldsymbol{M}$, and transforming the data from the cube to the head coordinate system. This is achieved by pre-multiplying the cube-to-global transformation matrix by the inverse of the head-to-global transformation matrix.

$M=K^{-1} T=\left[\begin{array}{cc}R & r_{H C} \\ 0 & 1\end{array}\right]$

Equation A-8

where $\boldsymbol{R}$ is a rotation matrix (3x3) that defines the orientation of the cube coordinate axes in the head coordinate system, and $\boldsymbol{r}_{\boldsymbol{H C}}$ is a vector $(3 \mathrm{x} 1)$ that points from the head origin to the cube origin in the head coordinate system. The rotation matrix, $\boldsymbol{R}$, can be used to transform the kinematics from the cube to the head coordinate system:

$$
\begin{aligned}
& {\left[\begin{array}{l}
\omega_{x} \\
\omega_{y} \\
\omega_{z}
\end{array}\right]=\boldsymbol{R}\left[\begin{array}{l}
\omega_{u} \\
\omega_{v} \\
\omega_{w}
\end{array}\right],} \\
& {\left[\begin{array}{l}
\alpha_{x} \\
\alpha_{y} \\
\alpha_{z}
\end{array}\right]=\boldsymbol{R}\left[\begin{array}{l}
\alpha_{u} \\
\alpha_{v} \\
\alpha_{w}
\end{array}\right],} \\
& {\left[\begin{array}{l}
a_{x, c} \\
a_{y, c} \\
a_{z, c}
\end{array}\right]=\boldsymbol{R}\left[\begin{array}{l}
a_{u} \\
a_{v} \\
a_{w}
\end{array}\right] .}
\end{aligned}
$$

Equation A-10

At this point, transformation of the rotational kinematics is complete; however, the linear accelerations are now defined at the cube origin in the head coordinate system. To transform the linear accelerations to the head origin requires Equation A-1, where the head origin is analogous to point $\mathrm{A}$ and cube origin, point $\mathrm{B}$ :

$$
a_{H}=a_{C}-\alpha \times r_{H C}-\omega \times\left(\omega \times r_{H C}\right)
$$

This concludes the calculations required to obtain 6DOF head kinematics in the local head coordinate system from NAP and 6DOF cubes. Table A-1 includes arm lengths 
and sensor seismic standoffs for the arrays used in this dissertation. A summary of the steps used to process the head kinematics data is shown in Figure 2-2.

Table A-1: Dimensions for NAP arm lengths and seismic standoffs.

\begin{tabular}{ccccccc}
\hline Dummy & $\boldsymbol{r}_{\mathbf{1}}(\mathrm{cm})$ & $\boldsymbol{r}_{2}(\mathrm{~cm})$ & $\boldsymbol{r}_{\mathbf{3}}(\mathrm{cm})$ & $\boldsymbol{s}_{\mathbf{1}}(\mathrm{mm})$ & $\boldsymbol{s}_{2}(\mathrm{~mm})$ & $\boldsymbol{s} \mathbf{3}(\mathrm{mm})$ \\
\hline $\begin{array}{c}\text { Polar II \& } \\
\text { Hybrid-III } \\
(\text { M50) }\end{array}$ & {$[5.59,0,0]$} & {$[0,4.83,0]$} & {$[0,0,8.13]$} & {$[5.15,0,0]$} & {$[0,5.15,0]$} & {$[0,0,5.15]$} \\
$\begin{array}{c}\text { THOR } \\
(\text { M50) }\end{array}$ & {$[-5.08,0,0]$} & {$[0,4.83,0]$} & {$[0,0,7.16]$} & N/A & N/A & N/A \\
$\begin{array}{c}\text { EuroSID 2RE } \\
(\text { M50) }\end{array}$ & {$[5.44,0,0]$} & {$[0,4.44,0]$} & {$[0,0,7.42]$} & N/A & N/A & N/A \\
$\begin{array}{c}\text { Hybrid-III } \\
(\text { F05 })\end{array}$ & {$[4.83,0,0]$} & {$[0,4.10,0]$} & {$[0,0,7.37]$} & N/A & N/A & N/A \\
$\begin{array}{c}\text { SID-2s } \\
(\text { F05 })\end{array}$ & {$[4.83,0,0]$} & {$[0,4.10,0]$} & {$[0,0,7.37]$} & N/A & N/A & N/A \\
\hline
\end{tabular}

Entries are $[x, y, z]$ components in the NAP coordinate system (Figure A-2).

Data Not Available (N/A). 


\section{Appendix B - Scaling Head Kinematics}

While the majority of head impacts were measured using a $50^{\text {th }}$ percentile, male test dummy, head impacts for several tests involved small females $\left(5^{\text {th }}\right.$ percentile $)$. To keep consistency with the FE models, head kinematic responses from the small female tests were scaled to a $50^{\text {th }}$ percentile male using the equal-stress equal-velocity relationship. This particular scaling technique was developed by Eppinger 1976, and assumes a linear relationship between length $(l)$, time $(t)$, and mass $(m)$ of a tested surrogate $(F)$ and standard reference $(M)$ (Yoganandan et al., 2014):

$$
\begin{aligned}
& \lambda_{l}=l_{M} / l_{F}, \\
& \lambda_{t}=t_{M} / t_{F}, \\
& \lambda_{m}=m_{M} / m_{F} .
\end{aligned}
$$

Equation B-1

Equation B-2

Equation B-3

For the $5^{\text {th }}$ female relative to a $50^{\text {th }}$ male, $\lambda_{l}=1.073$ (Mertz et al., 2003), which was used in the scaling relationships for the linear and angular accelerations, angular velocities, and time (Yoganandan et al., 2014):

$$
\begin{aligned}
& \boldsymbol{a}_{M}=\lambda_{l}^{-1} \boldsymbol{a}_{F}, \\
& \boldsymbol{\alpha}_{M}=\lambda_{l}^{-2} \alpha_{F}, \\
& \omega_{M}=\lambda_{l}^{-1} \omega_{F}, \\
& t_{M}=\lambda_{l} t_{F},
\end{aligned}
$$

Equation B-6

Equation B-7

where the subscript $F$ is for the female data and $M$, the male data. Other tests, which involved cadavers (male and female), were not scaled. 


\section{Appendix C - Filtering Sensitivity Study Results}

The section provides the results from the filtering sensitivity study (Section 2.1.2).

Ten separate head impacts were selected from the database (Table C-1), and filtered at four different low pass filter frequencies (Table C-2). Each head impact-filter combination was applied to both FE models to investigate the sensitivity of MPS and CSDM (Figure C-1).

Table C-1: Tests used in the filtering sensitivity study.

\begin{tabular}{|c|c|c|c|c|c|}
\hline $\begin{array}{c}\text { UVa } \\
\text { Database } \\
\text { ID }\end{array}$ & Test Type & Surrogate & $\begin{array}{c}\text { Sensor } \\
\text { Package }\end{array}$ & HIC & BrIC \\
\hline 1000 & PEDESTRIAN & $\begin{array}{l}\text { POLAR II, } \\
\text { M50 }\end{array}$ & NAP & 1.18 & 1314 \\
\hline 1015 & PEDESTRIAN & PMHS, F & $6 \mathrm{DOF}$ & 1.40 & 790 \\
\hline 1132 & NHTSA FRONTAL NCAP & $\begin{array}{l}\text { HYBRID III, } \\
\text { M50 }\end{array}$ & NAP & 0.61 & 431 \\
\hline 1388 & NHTSA $0^{\circ}$ PENDULUM & THOR, M50 & $6 \mathrm{DOF}$ & 0.52 & 1276 \\
\hline 1170 & NHTSA RD OBLIQUE & THOR, M50 & NAP & 0.63 & 504 \\
\hline 1417 & NHTSA $60^{\circ}$ PENDULUM & WSID, M50 & 6DOF & 1.00 & 591 \\
\hline 1235 & NHTSA SIDE NCAP & $\begin{array}{c}\text { EUROSID } 2 \\
\text { RE, M50 }\end{array}$ & NAP & 1.20 & 370 \\
\hline 1306 & NHTSA $90^{\circ}$ PENDULUM & $\begin{array}{c}\text { EUROSID } 2 \\
\text { RE, M50 }\end{array}$ & $6 \mathrm{DOF}$ & 0.88 & 509 \\
\hline 1648 & $\begin{array}{l}\text { IIHS MODERATE } \\
\text { OVERLAP }\end{array}$ & $\begin{array}{l}\text { HYBRID III, } \\
\text { M50 }\end{array}$ & $6 \mathrm{DOF}$ & 0.54 & 281 \\
\hline 1523 & $\begin{array}{l}\text { IIHS SMALL } \\
\text { OVERLAP }\end{array}$ & $\begin{array}{l}\text { HYBRID III, } \\
\text { M50 }\end{array}$ & $6 \mathrm{DOF}$ & 0.65 & 215 \\
\hline
\end{tabular}

Table C-2: Filter specifications.

\begin{tabular}{cc}
\hline $\begin{array}{c}\text { Channel Frequency } \\
\text { Class (CFC) }\end{array}$ & $\begin{array}{c}\text { Low Pass Filter } \\
\text { Frequency }(\mathrm{Hz})\end{array}$ \\
\hline 1000 & 1650 \\
180 & 300 \\
60 & 100 \\
- & 30 \\
\hline
\end{tabular}


(A) MPS $\left(95^{\text {th }}\right.$ percentile) GHBMC

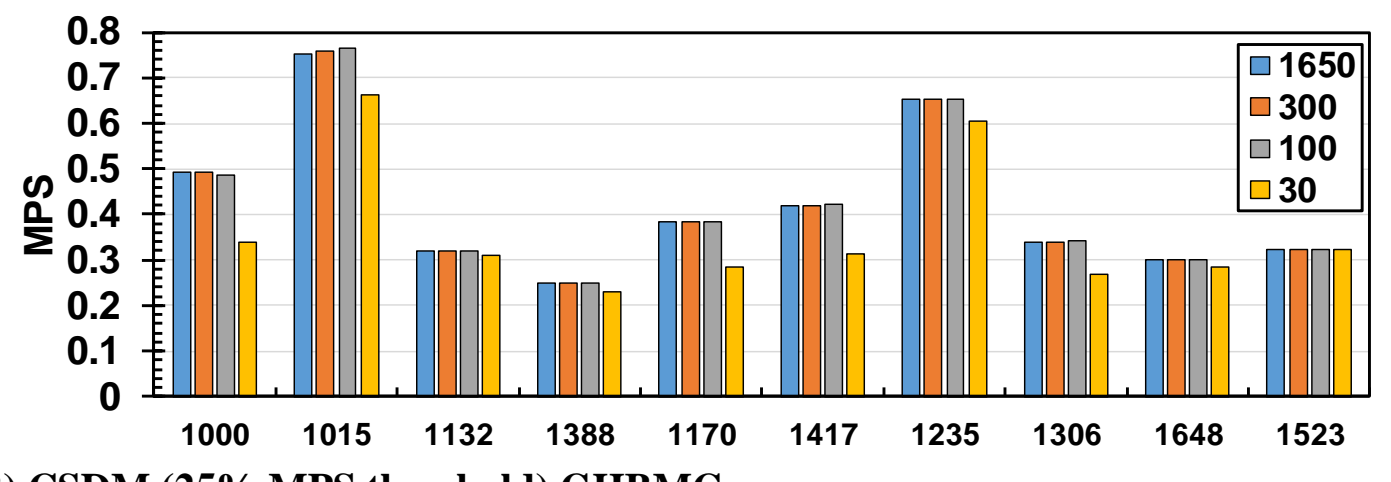

(B) CSDM (25\% MPS threshold) GHBMC

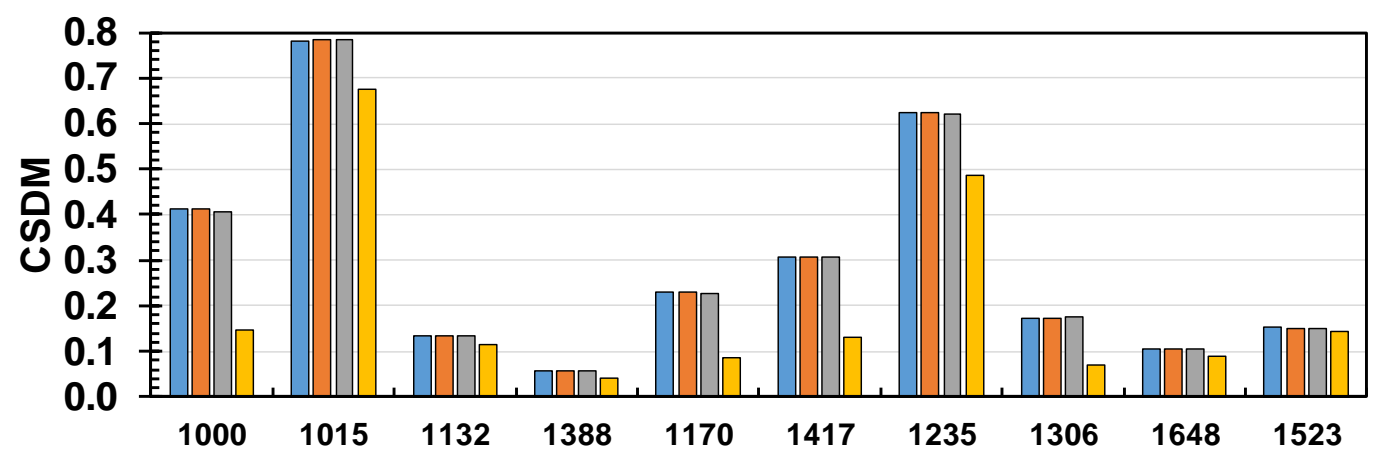

(C) MPS $\left(95^{\text {th }}\right.$ percentile) SIMon

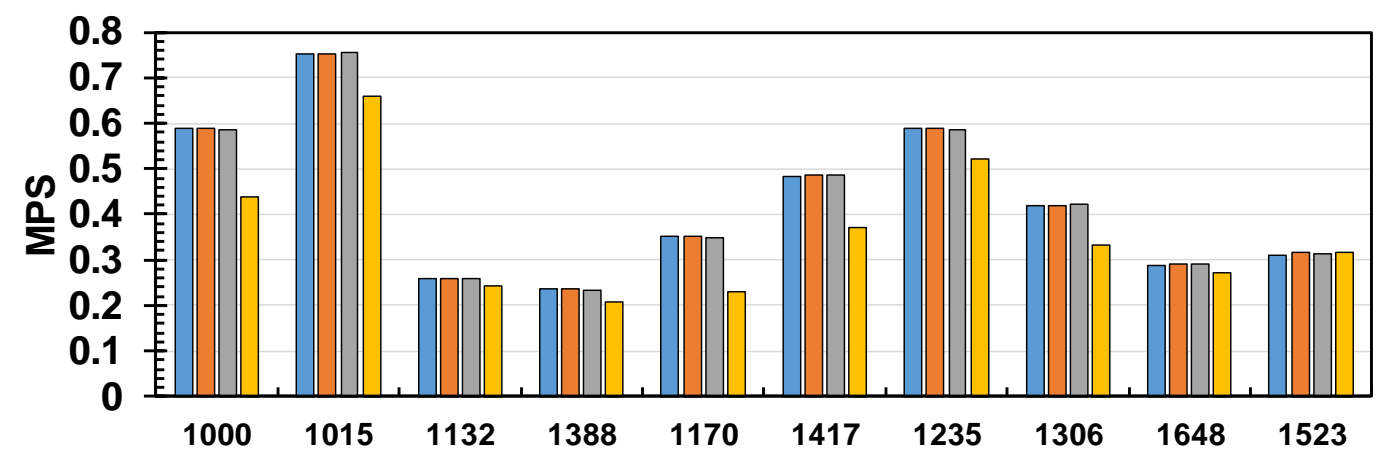

(D) CSDM (25\% MPS threshold) SIMon

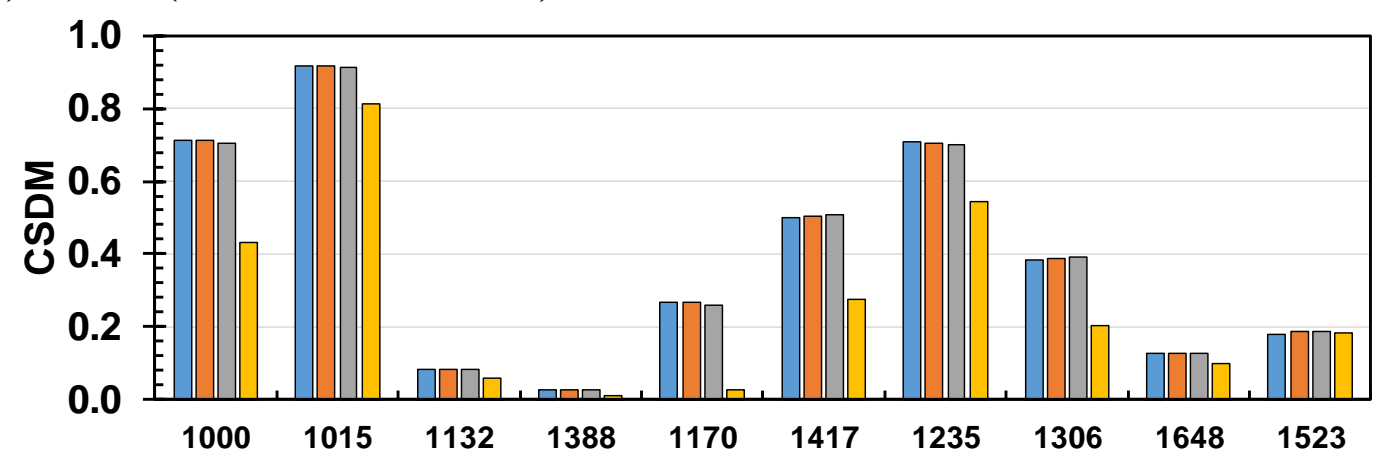


Figure C-1: MPS (A, C) and CSDM (B, D) sensitivity to low pass filter frequency $(\mathrm{Hz})$ for ten head impacts from the database (Table $\mathrm{C}-1)$. Results are based on $\operatorname{GHBMC}(\mathrm{A}, \mathrm{B})$ and SIMon $(\mathrm{C}, \mathrm{D})$.

(A) Results based on GHBMC

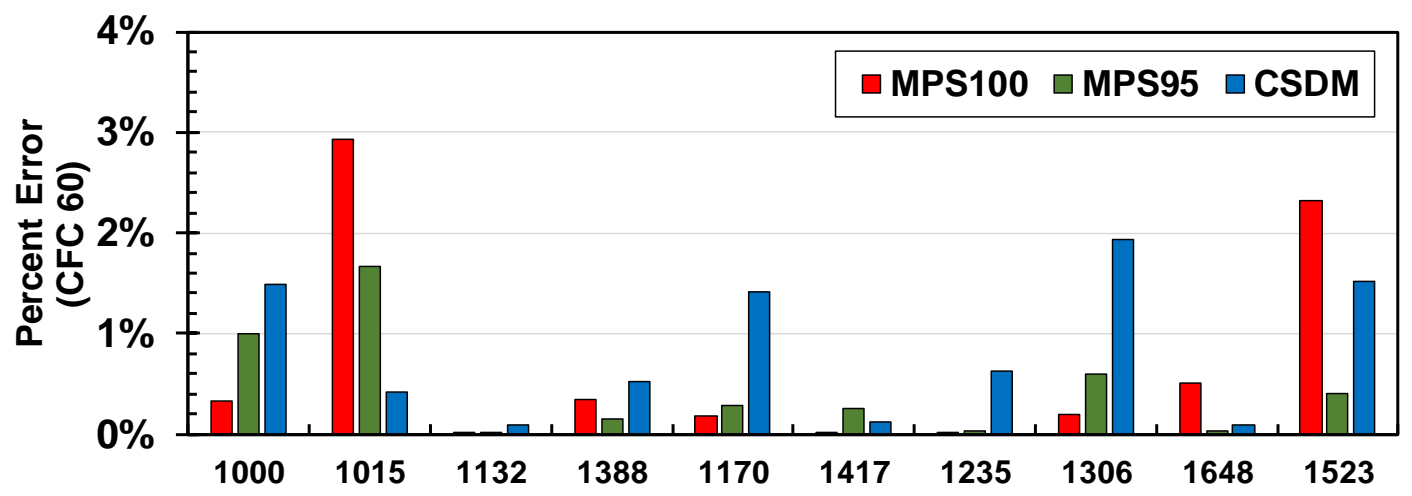

(B) Results based on SIMon

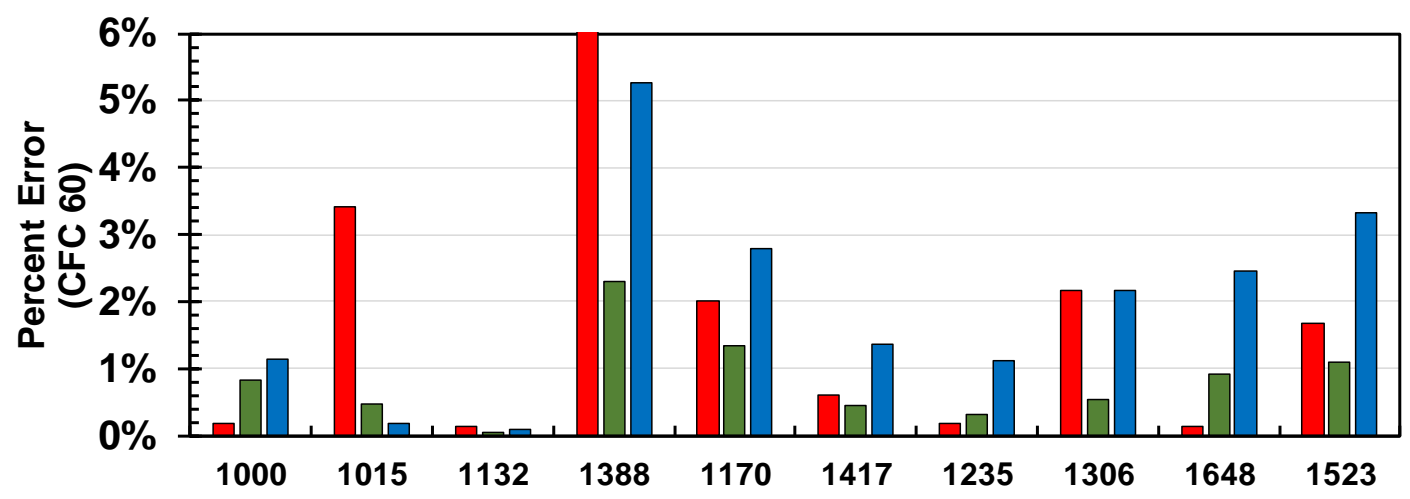

Figure C-2: Percent error in strain-based metrics at CFC 60 (recommended) relative to CFC 1000 (baseline). Less than $2.4 \%$ error occurred in MPS $\left(\mathbf{9 5}^{\text {th }}\right.$ percentile), and 5.2\% error in CSDM (25\% MPS threshold) relative to results from kinematics filtered at CFC 1000. Results are based on GHBMC (A) and SIMon (B). 


\section{Appendix D - FE Human Brain Model Specifications}

This section provides additional technical content on the FE human brain models used in this dissertation. Information provided includes a description of the various FE parts and their physical properties, material definitions, and contact/boundary conditions.

\section{D.1 GHBMC (v4.3)}

Table D-1: Mesh, mass, and density properties for GHBMC.

\begin{tabular}{|c|c|c|c|c|}
\hline Part & $\begin{array}{l}\text { Mesh } \\
\text { Type }\end{array}$ & $\begin{array}{c}\text { No. } \\
\text { Elements }\end{array}$ & $\begin{array}{l}\text { Mass } \\
(\mathrm{g})\end{array}$ & $\begin{array}{l}\text { Density } \\
\left(\mathrm{g} / \mathrm{cm}^{3}\right)\end{array}$ \\
\hline Cerebellum & $3 \mathrm{D}$ & 14280 & 123 & 1.06 \\
\hline Cerebrum Gray Lower & $3 \mathrm{D}$ & 5352 & 145 & 1.06 \\
\hline Cerebrum Gray Upper & $3 \mathrm{D}$ & 17348 & 346 & 1.06 \\
\hline Corpus Callosum & $3 \mathrm{D}$ & 980 & 20.9 & 1.06 \\
\hline Thalamus & $3 \mathrm{D}$ & 260 & 11.9 & 1.06 \\
\hline Ventricle Lateral & $3 \mathrm{D}$ & 1022 & 24.6 & 1.04 \\
\hline Brain Stem Midbrain & $3 \mathrm{D}$ & 504 & 9.82 & 1.06 \\
\hline Brain Stem & $3 \mathrm{D}$ & 2280 & 22.2 & 1.06 \\
\hline CSF Cerebrum & $3 \mathrm{D}$ & 9806 & 214 & 1.04 \\
\hline Basal Ganglia & $3 \mathrm{D}$ & 1284 & 26.1 & 1.06 \\
\hline Pia & $2 \mathrm{D}$ & 11790 & 48.9 & 1.10 \\
\hline Tentorium & $2 \mathrm{D}$ & 1348 & 8.75 & 1.10 \\
\hline CSF Cerebellum & $3 \mathrm{D}$ & 4671 & 60.9 & 1.04 \\
\hline Arachnoid Cerebrum & $2 \mathrm{D}$ & 9164 & 43.4 & 1.10 \\
\hline Arachnoid Cerebellum & $2 \mathrm{D}$ & 2874 & 9.93 & 1.10 \\
\hline Falx & $2 \mathrm{D}$ & 1163 & 9.40 & 1.10 \\
\hline Ventricle Third & $3 \mathrm{D}$ & 76 & 2.07 & 1.04 \\
\hline Sagittal Sinus & $3 \mathrm{D}$ & 780 & 4.19 & 1.04 \\
\hline Sagittal Sinus Anterior & $3 \mathrm{D}$ & 100 & 0.412 & 1.04 \\
\hline Sinus Dural & $2 \mathrm{D}$ & 538 & 3.39 & 1.10 \\
\hline Cerebrum White & $3 \mathrm{D}$ & 23340 & 455 & 1.06 \\
\hline Bridging Veins & $1 \mathrm{D}$ & 22 & 0.0364 & 1.13 \\
\hline Dura & $2 \mathrm{D}$ & 11726 & 997 & 1.10 \\
\hline Spinal Cap & $2 \mathrm{D}$ & 262 & 0.994 & 1.10 \\
\hline Total & - & 120970 & 2590 & - \\
\hline
\end{tabular}


Table D-2: Material property definitions for GHBMC.

\begin{tabular}{|c|c|c|c|c|c|c|}
\hline Part & $\begin{array}{l}\text { Bulk } \\
(K) \\
(\mathrm{GPa})\end{array}$ & $\begin{array}{l}\text { Poisson's } \\
(v)\end{array}$ & $\begin{array}{l}\text { Long- } \\
\text { Shear } \\
\left(G_{0}\right) \\
(\mathrm{GPa})\end{array}$ & $\begin{array}{l}\text { Short- } \\
\text { Shear } \\
\left(G_{1}\right) \\
(\mathrm{GPa})\end{array}$ & $\begin{array}{c}\text { Time }{ }^{f} \\
\left(\tau_{1}\right) \\
(\mathrm{ms})\end{array}$ & $\begin{array}{c}\text { Young's } \\
\qquad(E) \\
(\mathrm{GPa})\end{array}$ \\
\hline Cerebellum ${ }^{\mathrm{a}, \mathrm{e}}$ & 2.19 & - & $6.00 \mathrm{E}-06$ & $1.20 \mathrm{E}-06$ & $1.25 \mathrm{E}-02$ & - \\
\hline Cerebrum Gray ${ }^{\mathrm{a}, \mathrm{e}}$ & 2.19 & - & $6.00 \mathrm{E}-06$ & $1.20 \mathrm{E}-06$ & $1.25 \mathrm{E}-02$ & - \\
\hline Corpus Callosum ${ }^{\mathrm{a}, \mathrm{e}}$ & 2.19 & - & 7.50E-06 & $1.50 \mathrm{E}-06$ & $1.25 \mathrm{E}-02$ & - \\
\hline Thalamus a, e & 2.19 & - & $6.00 \mathrm{E}-06$ & $1.20 \mathrm{E}-06$ & $1.25 \mathrm{E}-02$ & - \\
\hline Ventricle Lateral a $^{\mathrm{a}}$ & 2.19 & - & $5.00 \mathrm{E}-07$ & $1.00 \mathrm{E}-07$ & $1.25 \mathrm{E}-02$ & - \\
\hline Brain Stem Midbrain ${ }^{\mathrm{a}, \mathrm{e}}$ & 2.19 & - & $1.20 \mathrm{E}-05$ & $2.40 \mathrm{E}-06$ & $1.25 \mathrm{E}-02$ & - \\
\hline Brain Stem ${ }^{\text {a, e }}$ & 2.19 & - & $1.20 \mathrm{E}-05$ & $2.40 \mathrm{E}-06$ & $1.25 \mathrm{E}-02$ & - \\
\hline CSF Cerebrum $^{\text {a }}$ & 2.19 & - & $5.00 \mathrm{E}-07$ & $1.00 \mathrm{E}-07$ & $1.25 \mathrm{E}-02$ & - \\
\hline Basal Ganglia ${ }^{\text {a, e }}$ & 2.19 & - & $6.00 \mathrm{E}-06$ & $1.20 \mathrm{E}-06$ & $1.25 \mathrm{E}-02$ & - \\
\hline $\mathrm{Pia}^{\mathrm{b}}$ & - & 0.35 & - & - & - & $1.25 \mathrm{E}-02$ \\
\hline Tentorium $^{\mathrm{b}}$ & - & 0.30 & - & - & - & $3.15 \mathrm{E}-02$ \\
\hline CSF Cerebellum $^{\mathrm{a}}$ & 2.19 & - & $3.00 \mathrm{E}-06$ & $6.00 \mathrm{E}-07$ & $1.25 \mathrm{E}-02$ & - \\
\hline Arachnoid Cerebrumb & - & 0.35 & - & - & - & $1.20 \mathrm{E}-02$ \\
\hline Arachnoid Cerebellum ${ }^{\mathrm{b}}$ & - & 0.35 & - & - & - & $1.20 \mathrm{E}-02$ \\
\hline Falx $^{\mathrm{b}}$ & - & 0.35 & - & - & - & $1.25 \mathrm{E}-02$ \\
\hline Ventricle Third ${ }^{\mathrm{a}}$ & 2.19 & - & $5.00 \mathrm{E}-07$ & $1.00 \mathrm{E}-07$ & $1.25 \mathrm{E}-02$ & - \\
\hline Sagittal Sinus ${ }^{a}$ & 2.19 & - & $5.00 \mathrm{E}-07$ & $1.00 \mathrm{E}-07$ & $1.25 \mathrm{E}-02$ & - \\
\hline Sagittal Sinus Anterior ${ }^{a}$ & 2.19 & - & $5.00 \mathrm{E}-07$ & $1.00 \mathrm{E}-07$ & $1.25 \mathrm{E}-02$ & - \\
\hline Sinus Dural ${ }^{\mathrm{b}}$ & - & 0.35 & - & - & - & $3.15 \mathrm{E}-02$ \\
\hline Cerebrum White ${ }^{\text {a, e }}$ & 2.19 & - & 7.50E-06 & $1.50 \mathrm{E}-06$ & $1.25 \mathrm{E}-02$ & - \\
\hline Bridging Veins ${ }^{c}$ & - & 0.48 & - & - & - & $3.00 \mathrm{E}-02$ \\
\hline Dura $^{\mathrm{d}}$ & - & 0.35 & - & - & - & $3.15 \mathrm{E}-02$ \\
\hline Spinal Cap ${ }^{b}$ & - & 0.32 & - & - & - & $3.15 \mathrm{E}-02$ \\
\hline
\end{tabular}

Table D-3: Contact and boundary definitions for GHBMC.

\begin{tabular}{|c|c|c|}
\hline Brain Stem Boundary & $\begin{array}{c}\text { Skull-Meninges-CSF-Brain } \\
\text { Contact }\end{array}$ & 6DOF kinematics \\
\hline $\begin{array}{l}\text { Shared nodes between } \\
\text { foramen and brain stem }\end{array}$ & $\begin{array}{l}\text { No skull; shared nodes } \\
\text { between meninges, CSF, and } \\
\text { brain; Falx-Tentorium: } \\
\text { *CONTACT_TIED_SHELL_- } \\
\text { EDGE_TO_SURFACE_BEA } \\
\text { M_OFFSET }\end{array}$ & $\begin{array}{c}\text { Rigid Dura: } \\
\text { *BOUNDARY_PRESCRIBED_ } \\
\text { MOTION_RIGID_LOCAL }\end{array}$ \\
\hline
\end{tabular}




\section{D.2 SIMon (v4.0)}

Table D-4: Mesh, mass, and density properties for SIMon.

\begin{tabular}{ccccc}
\hline Part & $\begin{array}{c}\text { Mesh } \\
\text { Type }\end{array}$ & $\begin{array}{c}\text { No. } \\
\text { Elements }\end{array}$ & $\begin{array}{c}\text { Mass } \\
(\mathrm{g})\end{array}$ & $\begin{array}{c}\text { Density } \\
\left(\mathrm{g} / \mathrm{cm}^{3}\right)\end{array}$ \\
\hline Cerebrum & 3D & 20622 & 969 & 1.04 \\
Cerebellum & 3D & 7564 & 101 & 1.04 \\
Brainstem & 3D & 1944 & 16.6 & 1.04 \\
Ventricle & 3D & 390 & 10.9 & 1.00 \\
Vessels & 1D & 14 & 0.002 & 5.00 \\
Falx-Tent & 2D & 1251 & 24.8 & 1.13 \\
CSF & 3D & 10188 & 382 & 1.05 \\
Skull & 3D & 3790 & 3000 & 35.2 \\
Foramen & 3D & 112 & 0.128 & 1.05 \\
\hline Total & - & $\mathbf{4 5 8 7 5}$ & $\mathbf{4 5 0 4}$ & -
\end{tabular}

Table D-5: Material property definitions for SIMon.

\begin{tabular}{|c|c|c|c|c|c|c|}
\hline Part & $\begin{array}{l}\text { Bulk } \\
(K) \\
(\mathrm{GPa})\end{array}$ & $\begin{array}{l}\text { Poisson's } \\
\quad(v)\end{array}$ & $\begin{array}{l}\text { Long- } \\
\text { Shear } \\
\left(G_{0}\right) \\
(\mathrm{GPa})\end{array}$ & $\begin{array}{l}\text { Short- } \\
\text { Shear } \\
\left(G_{1}\right) \\
(\mathrm{GPa})\end{array}$ & $\begin{array}{l}\text { Decay }^{\mathrm{g}} \\
\left(\beta_{1}\right) \\
(1 / \mathrm{ms})\end{array}$ & $\begin{array}{l}\text { Young's } \\
\qquad(E) \\
(\mathrm{GPa})\end{array}$ \\
\hline Cerebrum $^{\mathrm{a}, \mathrm{f}}$ & 0.558 & - & $9.28 \mathrm{E}-07$ & $1.66 \mathrm{E}-06$ & $1.70 \mathrm{E}-02$ & - \\
\hline Cerebellum ${ }^{\mathrm{a}, \mathrm{f}}$ & 0.558 & - & $9.28 \mathrm{E}-07$ & $1.66 \mathrm{E}-06$ & $1.70 \mathrm{E}-02$ & - \\
\hline Brainstem $^{\mathrm{a}, \mathrm{f}}$ & 0.558 & - & $9.28 \mathrm{E}-07$ & $1.66 \mathrm{E}-06$ & $1.70 \mathrm{E}-02$ & - \\
\hline Ventricle $^{\mathrm{b}}$ & 2.10 & - & - & - & - & - \\
\hline Vessels ${ }^{c}$ & - & - & - & - & - & $2.75 \mathrm{E}-04$ \\
\hline Falx-Tent ${ }^{\mathrm{d}}$ & - & 0.45 & - & - & - & $3.15 \mathrm{E}-02$ \\
\hline $\mathrm{CSF}^{\mathrm{a}}$ & 0.005 & - & $1.00 \mathrm{E}-04$ & $2.00 \mathrm{E}-05$ & $1.00 \mathrm{E}-05$ & - \\
\hline Skull $\mathrm{e}^{\mathrm{e}}$ & - & 0.30 & - & - & - & 6.90 \\
\hline Foramen $^{\mathrm{d}}$ & - & 0.45 & - & - & - & 6.93 \\
\hline \multicolumn{7}{|c|}{$\begin{array}{l}{ }^{a} \text { MAT_KELVIN-MAXWELL_VISCOELASTIC; }{ }^{\mathrm{b}} \text { MAT_ELASTIC_FLUID; } \\
\text { c MAT_CABLE_DISCRETE_BEAM; }{ }^{\mathrm{d}} \text { MAT_ELASTIC; }{ }^{\mathrm{e}} \text { MAT_RIGID; } \\
{ }^{\mathrm{f}} \text { Brain parts used in the calculation of strain-based metrics. } \\
\text { g Maxwell formulation option in LS-DYNA }(\mathrm{FO}=0) \text {. }\end{array}$} \\
\hline \multicolumn{7}{|c|}{ Table D-6: Contact and boundary definitions for SIMon. } \\
\hline \multicolumn{2}{|c|}{ Brain Stem Boundary } & \multicolumn{2}{|c|}{$\begin{array}{l}\text { Skull-Meninges-CSF-Brain } \\
\text { Contact }\end{array}$} & \multicolumn{3}{|c|}{ 6DOF kinematics } \\
\hline \multicolumn{2}{|c|}{$\begin{array}{l}\text { Shared nodes between } \\
\text { foramen and brain stem }\end{array}$} & \multicolumn{2}{|c|}{$\begin{array}{l}\text { Shared nodes between all parts } \\
\text { (Meninges not distinguished) }\end{array}$} & \multicolumn{3}{|c|}{$\begin{array}{c}\text { Rigid Skull: } \\
\text { *BOUNDARY_PRESCRIBED_ } \\
\text { MOTION_RIGID_LOCAL }\end{array}$} \\
\hline
\end{tabular}




\section{Appendix E - Calculation of CSDM}

This section provides additional details on the calculation of the Cumulative Strain Damage Measure (CSDM) strain-based metric from the FE brain models. The metric was originally proposed as an indicator of DAI, where the cumulative strain damage is believed to be related to axonal damage accumulated during the impact event. The metric is calculated for an arbitrary element maximum principal strain threshold, $\varepsilon_{T}$, as

$C S D M=\sum_{i}^{n} \frac{V_{i}}{V_{t o t}}$, for $\varepsilon_{i} \geq \varepsilon_{T}$,

Equation E-1

where $\varepsilon_{i}$ is the maximum principal strain and $V_{i}$ is the volume of the $i^{\text {th }}$ element in the FE brain model. The total volume of the brain model is $V_{\text {tot }}$ :

$V_{\text {tot }}=\sum_{i}^{n} V_{i}$

Equation E-2

where $n$ is the total number of brain elements in the FE model. Only parts that use 3D solid elements and the *MAT_KELVIN-MAXWELL_VISCOELASTIC material card (LSDYNA) were included in the calculation of MPS and CSDM. These parts are indicated in Appendix D, Tables D-2 and D-5. Values for the total brain volume for GHBMC and SIMon are provided in section 2.2.1 and Table E-1.

Table E-1: FE model brain volume specifications for CSDM calculation.

\begin{tabular}{cccc}
\hline Model & $\begin{array}{c}\text { Total brain volume } \\
\left(\mathrm{cm}^{3}\right)\end{array}$ & $\begin{array}{c}\text { Element volume } \\
\text { Avg } \pm \text { S.D. }\left(\mathrm{mm}^{3}\right)\end{array}$ & $\begin{array}{c}\text { Total \# of elements } \\
\text { used in calculation }\end{array}$ \\
\hline GHBMC & 1094 & $16.7 \pm 12.5$ & 65628 \\
SIMon & 1045 & $34.7 \pm 30.1$ & 30130 \\
\hline
\end{tabular}




\section{Appendix F - Solutions to the sDOF mechanical system}

This section presents the closed-form solutions to the response of the sDOF model to different base excitation pulses. Only analytical pulse shapes are investigated, and model response to both damped and undammed motion are explored.

\section{F.1 Equation of Motion of the sDOF Model}

Consider the following sDOF mechanical system with base excitation (Figure F-1). The equations of motion (EOM) for this system can be written using Newton's $2^{\text {nd }}$ Law of Motion (Equation F-1):

(A)

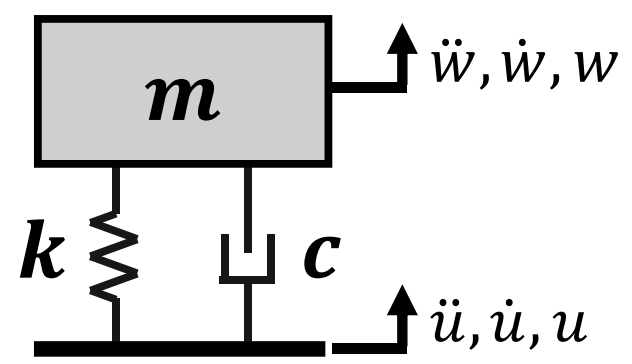

(B)

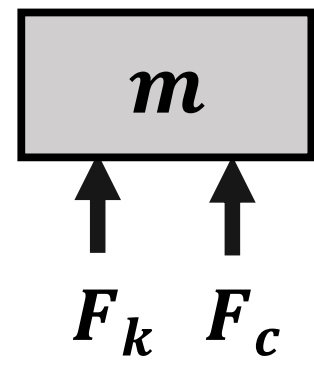

Figure F-1: sDOF mechanical system (A) and free body diagram (B). The arrow at the right indicates the direction of positive motion.

$m \ddot{w}=F_{c}+F_{k}=c(\dot{u}-\dot{w})+k(u-w)$

Equation F-1

where $\ddot{u}, \dot{u}, u$ and $\ddot{w}, \dot{w}, w$ are the accelerations, velocities, and displacements of the base and mass, $m$, while $c$ and $k$ are the system parameters for the damping, and stiffness, respectively. In its current form, Equation F-1 consists of two degrees of freedom ( $u$ and $w$ ) however, this can be reduced to a single DOF system by substituting in for the relative base-mass motion, $\delta=u-w$ :

$m \ddot{\delta}+c \dot{\delta}+k \delta=m \ddot{u}$.

Equation F-2 
It is worth pointing out that Equation F-2 is analogous to the EOM of a fixed-base, forceexcited system, where $m \ddot{u}=F$, and $F$ is the force applied to the mass. With respect to the system shown in Figure F-1, A, F is considered a pseudo force, while $\ddot{u}$ is considered a pseudo acceleration in the fixed-base analog. Equation F-2 can be further simplified by dividing both sides by $m$, and substituting the natural frequency, $\omega_{n}$, and damping ratio, $\zeta$ :

$\omega_{n}=\sqrt{k m^{-1}}$

Equation F-3

$\zeta=c / \sqrt{4 m k}$,

Equation F-4

$\ddot{\delta}+2 \zeta \omega_{n} \dot{\delta}+\omega_{n}^{2} \delta=\ddot{u}$

Equation F-5

Equation F-5, can also be expressed in terms of the natural period, $\Delta t_{n}$ of the system:

$\ddot{\delta}+4 \pi \zeta \Delta t_{n}^{-1} \dot{\delta}+4 \pi^{2} \Delta t_{n}^{-2} \delta=\ddot{u}$, Equation F-6

where

$\Delta t_{n}=2 \pi / \omega_{n}=2 \pi \sqrt{m k^{-1}}$

Equation F-7

In the following subsections, the analytical solution to the time history of the response, $\delta=\delta(t)$, is determined for several idealized pulse shapes. Of particular interest is the solution to maximum magnitude of the response:

$\delta_{m}=\max _{t}\{|\delta(t)|\}$

Equation F-8

which is used in this dissertation as an analog for maximum brain deformation (MPS correlate). Thus, the purpose of these subsections is to determine a closed-form solution that relates pulse parameters (velocity and acceleration) to the maximum magnitude of the sDOF model response $\left(\delta_{m}\right)$. In this dissertation, the sDOF model is assumed to be underdamped $(0<\zeta<1)$. 


\section{F.2 Analytical Solution for a Square Pulse}

This analysis begins with the square pulse (Figure F-2), since it is the simplest analytical shape (constant) that can be used for a particular solution to the EOM (Equation F-5). The expression for the sDOF system with a unit step in acceleration is:

$\ddot{\delta}(t)+2 \zeta \omega_{n} \dot{\delta}(t)+\omega_{n}^{2} \delta(t)=\ddot{u}(t)=\ddot{u}_{m}\left\{\begin{array}{cc}1 & 0 \leq t<\Delta t \\ 0 & \Delta t \leq t\end{array}\right.$,

Equation F-9

Where $\ddot{u}_{m}$ is the magnitude of the step, and $\Delta t$ is the duration of the pulse.

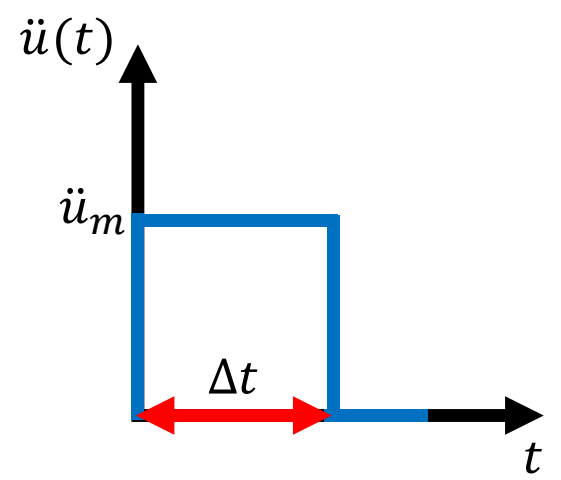

Figure F-2: Unit step acceleration pulse.

The solution to Equation F-9 requires solving the ordinary differential equation (ODE) in two separate time regimes. For $0 \leq t<\Delta t$, the response, $\delta=\delta_{1}(t)$, is a wellknown solution in mechanical vibrations from which over-shoot can be analyzed:

$\delta_{1}(t)=A_{1}\left[1-\frac{e^{-\zeta \omega_{n} t}}{\sqrt{1-\zeta^{2}}} \cos \left(\omega_{d} t-\varphi_{1}\right)\right]$

Equation F-10

where the initial conditions are assumed zero: $\delta_{1}(0)=\dot{\delta}_{1}(0)=0$, and

$$
\begin{aligned}
& A_{1}=\frac{\ddot{u}_{m}}{\omega_{n}^{2}}, \\
& \varphi_{1}=\tan ^{-1}\left(\frac{\zeta}{\sqrt{1-\zeta^{2}}}\right), \\
& \omega_{d}=\omega_{n} \sqrt{1-\zeta^{2}},
\end{aligned}
$$


are the amplitude and relative phase of the forced vibration response, and damped natural frequency, respectively (Rao and Yap, 1995). For $\Delta t \leq t$, the response, $\delta=\delta_{2}(t)$, is a free vibration, where the initial conditions are determined by satisfying the corresponding boundary conditions: $\delta_{2}(\Delta t)=\delta_{1}(\Delta t)=\delta_{\Delta t}$ and $\dot{\delta}_{2}(\Delta t)=\dot{\delta}_{1}(\Delta t)=\dot{\delta}_{\Delta t}$, which leads to the following solution:

$\delta_{2}(t)=A_{2} e^{-\zeta \omega_{n}(t-\Delta t)} \cos \left(\omega_{d}(t-\Delta t)-\varphi_{2}\right)$, Equation F-14 where

$A_{2}=\frac{\sqrt{\delta_{\Delta t}^{2} \omega_{n}^{2}+\dot{\delta}_{\Delta t}{ }^{2}+2 \delta_{\Delta t} \dot{\delta}_{\Delta t} \zeta \omega_{n}}}{\omega_{d}}$ Equation F-15

$\varphi_{2}=\tan ^{-1}\left(\frac{\dot{\delta}_{\Delta t}+\zeta \omega_{n} \delta_{\Delta t}}{\delta_{\Delta t} \omega_{d}}\right)$

Equation F-16

and

$\delta_{\Delta t}=A_{1}\left[1-\frac{e^{-\zeta \omega_{n} \Delta t}}{\sqrt{1-\zeta^{2}}} \cos \left(\omega_{d} \Delta t-\varphi_{1}\right)\right]$

Equation F-17

$\dot{\delta}_{\Delta t}=A_{1} \frac{e^{-\zeta \omega_{n} \Delta t}}{\sqrt{1-\zeta^{2}}}\left[\zeta \omega_{n} \cos \left(\omega_{d} \Delta t-\varphi_{1}\right)+\omega_{d} \sin \left(\omega_{d} \Delta t-\varphi_{1}\right)\right]$

Equation F-18

are the amplitude, phasing, and initial conditions of the free vibration response, respectively (Rao and Yap, 1995). The total response of the system is then

$\delta(t)=\left\{\begin{array}{cc}\delta_{1}(t) & 0 \leq t<\Delta t \\ \delta_{2}(t) & \Delta t \leq t\end{array}\right\}$

Equation F-19

To obtain a closed-form solution to the maximum response of the system $\left(\delta_{m}\right)$ involves determining the time to peak, analytically and substituting back into Equation F19. Let's first examine the solution in the region $0 \leq t<\Delta t$ (Equation F-10). Finding the time to peak involves taking the derivative of the response and setting equal to zero:

$\dot{\delta}_{1}\left(t_{p}\right)=A_{1} \frac{e^{-\zeta \omega_{n} t_{p}}}{\sqrt{1-\zeta^{2}}}\left[\zeta \omega_{n} \cos \left(\omega_{d} t_{p}-\varphi_{1}\right)+\omega_{d} \sin \left(\omega_{d} t_{p}-\varphi_{1}\right)\right]=0 . \quad$ Equation $\mathbf{F}-20$ 
Equation F-20 is satisfied when $\omega_{d} t_{p}=n_{k} \pi$, for $n_{k}=1,2,3 \ldots$ Thus, the first peak is

$t_{p}=\pi / \omega_{d}$

Equation F-21

which also happens to be the largest peak, since damping will attenuate the magnitude of subsequent peaks, i.e., $n_{k}>1$. Substituting Equation F-21 into Equation F-10 results in the familiar equation for overshoot:

$\delta_{1 m}=\delta_{1}\left(t_{p}\right)=A_{1}\left[1+e^{\frac{-\zeta \pi}{\sqrt{1-\zeta^{2}}}}\right]$.

Equation F-22

It is important to note that Equation F-22 depends only on the magnitude of the pulse and not on its duration; however, it is valid only when $\Delta t>\pi / \omega_{d}$.

Let's now examine the solution in the region $\Delta t \leq t$ (Equation F-14). Finding the time to peak, again, involves taking the derivative of the response and setting equal to zero: $\dot{\delta}_{2}\left(t_{p}\right)=A_{2} e^{-\zeta \omega_{n}\left(t_{p}-\Delta t\right)}\left[\begin{array}{c}\zeta \omega_{n} \cos \left(\omega_{d}\left(t_{p}-\Delta t\right)-\varphi_{2}\right) \ldots \\ +\omega_{d} \sin \left(\omega_{d}\left(t_{p}-\Delta t\right)-\varphi_{2}\right)\end{array}\right]=0$

Equation F-23

which simplifies to

$t_{p}=\frac{\varphi_{2}-\varphi_{1}}{\omega_{d}}+\Delta t$

Equation F-24

Substituting Equation F-24 into Equation F-14 for time $t$ yields:

$\delta_{2 m}=\delta_{2}\left(t_{p}\right)=A_{2} \sqrt{1-\zeta^{2}} e^{\frac{-\zeta}{\sqrt{1-\zeta^{2}}}\left(\varphi_{2}-\varphi_{1}\right)}$.

Equation F-25

Thus the maximum response for a square pulse for any magnitude and duration can be expressed as

$\delta_{m}=\left\{\begin{array}{ll}\delta_{2 m} & \Delta t \leq \pi / \omega_{d} \\ \delta_{1 m} & \Delta t>\pi / \omega_{d}\end{array}\right\}$

Equation F-26 
Upon examining Equation F-26 it is evident that solution is mathematically cumbersome; however, if damping is neglected the solution reduces to a mathematical form that is more manageable to analyze:

$\delta_{m}^{\prime}=\left.\delta_{m}\right|_{\zeta=0}=\left\{\begin{array}{cc}\delta_{2 m}^{\prime}=A_{1} \sqrt{2\left(1-\cos \left(\omega_{n} \Delta t\right)\right)} & \Delta t \leq \pi / \omega_{n} \\ \delta_{1 m}^{\prime}=2 A_{1} & \Delta t>\pi / \omega_{n}\end{array}\right\}$.

Equation F-27

which is the result obtained by Yanaoka et al., 2015. Taking the limit of Equation F-27 when the duration is small $\left(\Delta t \ll \pi / \omega_{n}\right)$, and omitting higher order terms, $\mathcal{O}\left(\Delta t^{\prime 2}\right)$, gives the following result:

$\lim _{\Delta t^{\prime} \rightarrow 0} \delta_{2 m}^{\prime}=\frac{\ddot{u}_{m}}{\omega_{n}} \Delta t$

Equation F-28

where

$\Delta t^{\prime}=\omega_{n} \Delta t$

Equation F-29

and the Taylor series expansion of cosine is used:

$\cos \left(\Delta t^{\prime}\right)=1-\frac{\left(\Delta t^{\prime}\right)^{2}}{2}+\mathcal{O}\left(\Delta t^{\prime 4}\right) \approx 1-\frac{\left(\Delta t^{\prime}\right)^{2}}{2}$

Equation F-30

Based on Equation F-28, when the duration is small the maximum response depends on the magnitude of the pulse times the duration, i.e., the change in velocity, which for the case of zero initial conditions is equivalent to the magnitude of the pulse integral (velocity): $\ddot{u}_{m} \Delta t=\dot{u}_{m}$.

Equation F-31

A similar result is obtained for the damped system:

$\lim _{\Delta t \rightarrow 0} \delta_{2 m}=\frac{\ddot{u}_{m} \Delta t}{\omega_{n}} \cdot \exp \left[\frac{-\zeta \cos ^{-1} \zeta}{\sqrt{1-\zeta^{2}}}\right]=\frac{\dot{u}_{m}}{\omega_{n}} \cdot \exp \left[\frac{-\zeta \cos ^{-1} \zeta}{\sqrt{1-\zeta^{2}}}\right]$

Equation F-32

Thus, based on the square pulse: for short duration pulses, maximum deformation depends on the change in velocity only (Equation F-31), while for long duration pulses, maximum deformation depends on the magnitude of acceleration only (Equation F-22). For pulses 
near the natural period, maximum deformation depends on two of the three pulse parameters; duration, acceleration, and/or velocity (Equation F-26 for the damped system, and Equation F-27; $\Delta t \leq \pi / \omega_{n}$, for the undamped system). It is important to note that the transition from short-to-long duration is not continuous and requires two separate functions. For the square pulse, this switch over occurs at the natural and damped natural periods of vibration, which can be defined as:

$\Delta t_{n}=\pi / \omega_{n}$,

Equation F-32

$\Delta t_{d}=\pi / \omega_{d}$

Equation F-33

While Equations F-32 and F-33 contradict Equation F-7, defining the natural and damped period in this manner simplifies the mathematics and interpretation of the results. One way to define this switch analytically is to use Heaviside step functions. Rewriting Equations F-26 and F-27 in terms of the Heaviside function, $H(t)$ gives the result:

$\delta_{m}=\delta_{2 m} H\left(\Delta t_{d}-\Delta t\right)+\delta_{1 m} H\left(\Delta t-\Delta t_{d}\right)$

Equation F-34

$\delta_{m}^{\prime}=\delta_{2 m}^{\prime} H\left(\Delta t_{n}-\Delta t\right)+\delta_{1 m}^{\prime} H\left(\Delta t-\Delta t_{n}\right)$

Equation F-35

and, upon inspection it can be verified that, $\delta_{2 m}\left(\Delta t=\Delta t_{d}\right)=\delta_{1 m}\left(\Delta t=\Delta t_{d}\right)$ and $\delta_{2 m}^{\prime}\left(\Delta t=\Delta t_{n}\right)=\delta_{1 m}^{\prime}\left(\Delta t=\Delta t_{n}\right)$. Finally, given values for system parameters, Equations F-34 and F-35 can be solved for a broad range of pulse parameters (Figure F-3): For a square pulse, when the duration is less than the natural and damped periods, the time to peak happens beyond the pulse duration; $\Delta t<t_{p}<\Delta t_{n}, \Delta t_{d}$ (Figure F-3, top row). When the duration is greater than the natural and damped periods, the response becomes duration independent, and the time to peak happens within the pulse duration, and at the natural and damped periods; $t_{p}=\Delta t_{n}, \Delta t_{d}<\Delta t$ (Figure F-3, bottom row). The switch between these regions happens at the natural and damped periods (Figure F-3, middle rows, respectively). 

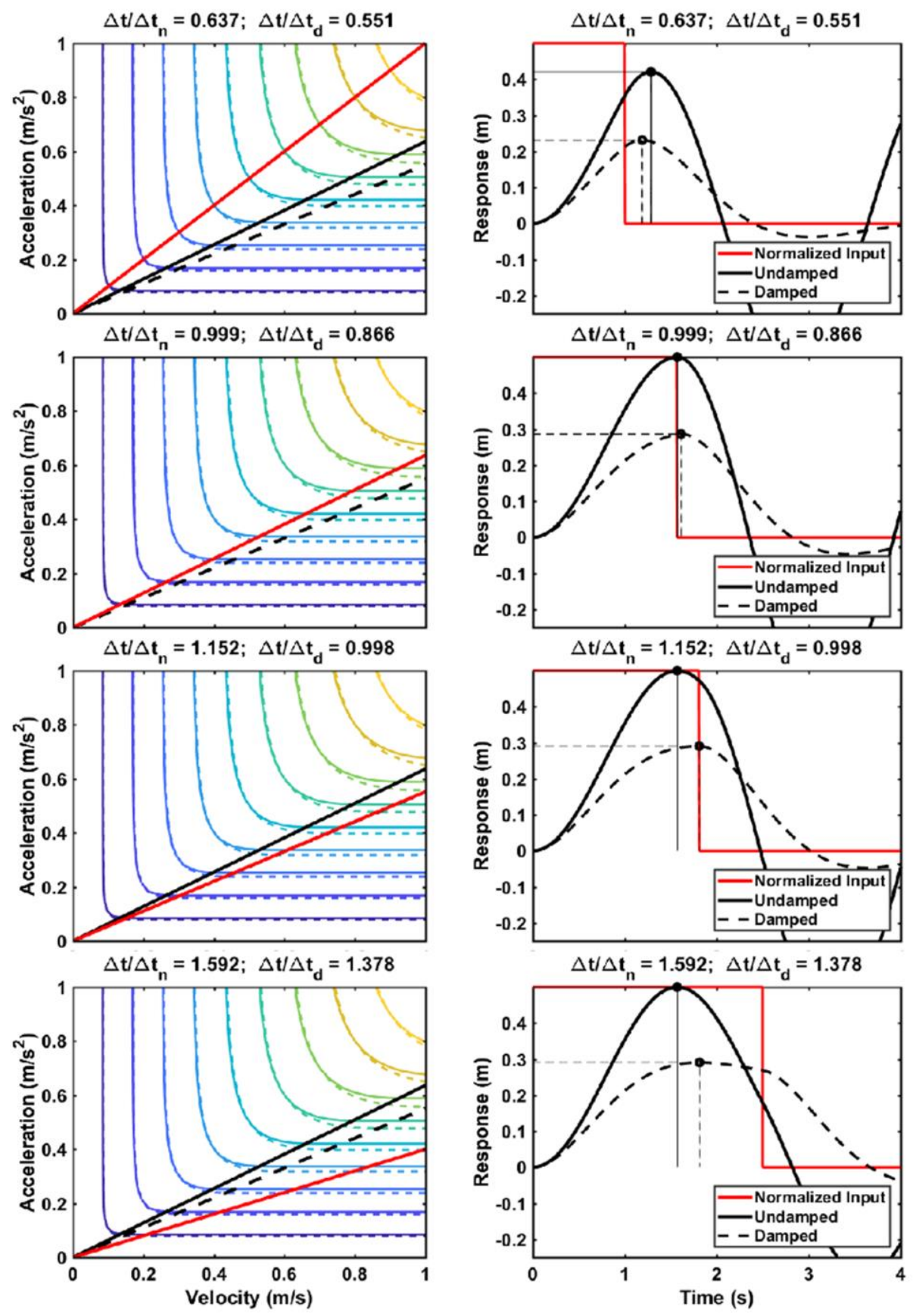

Figure F-3: Contours of maximum deformation for the damped (dotted contours, $\delta_{m}$ ), and undamped (solid contours, $\delta_{m}^{\prime}$ ) sDOF model subjected to a broad range of square pulses (left column). Solid and dotted diagonal lines on the contours are the natural and damped periods, respectively. Contours were normalized by the maximum value in the plot. Time histories for damped and undamped responses

to a square pulse with duration that corresponds to the solid red line on the contours (right column). The input pulses were normalized by the magnitude of the undamped response (right column). For this exercise $\omega_{n}=2$ and $\zeta=0.5$. 


\section{F.3 Analytical Solution for a Sinusoidal Pulse}

In this subsection both half and full cycle sinusoid pulses are examined. The expression for the $\mathrm{SDOF}$ system with sinusoidal acceleration is:

$\ddot{\delta}(t)+2 \zeta \omega_{n} \dot{\delta}(t)+\omega_{n}{ }^{2} \delta(t)=\ddot{u}_{m}\left\{\begin{array}{cc}\sin \left(\frac{n_{c} \pi t}{\Delta t}\right) & 0 \leq t<\Delta t \\ 0 & \Delta t \leq t\end{array}\right.$

Where $\ddot{u}_{m}$ is the magnitude of the pulse, and $\Delta t$ is the duration of the pulse. The span of the pulse is indicated by $n_{c} ; n_{c}=1$ for half cycle, and $n_{c}=2$ for full cycle (Equation 4-4).

(A)

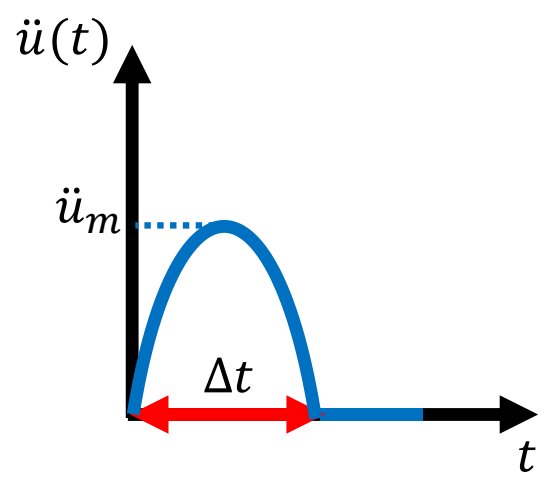

(B)

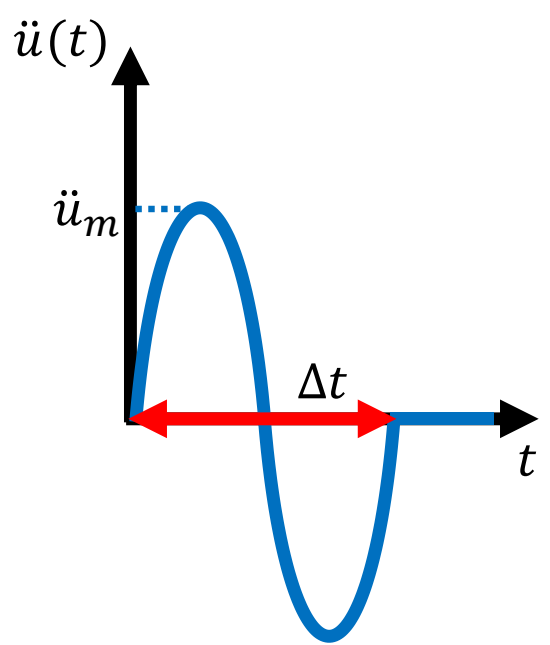

Figure F-4: Half (A) and Full (B) cycle sinusoid acceleration pulses.

The solution to Equation F-36, again requires solving the ODE in two separate time regimes. For $0 \leq t<\Delta t$, the response, $\delta=\delta_{1}(t)$, is a forced vibration:

$\delta_{1}(t)=\widetilde{A_{1}}\left\{\begin{array}{c}\sin \left(\Omega t-\widetilde{\varphi_{1}}\right)+\cdots \\ \left.-\zeta \omega_{n} t\left[\sin \widetilde{\varphi_{1}} \cos \left(\omega_{d} t\right)+\left(\frac{\zeta \omega_{n} \sin \widetilde{\varphi_{1}}-\Omega \cos \widetilde{\varphi_{1}}}{\omega_{d}}\right) \sin \left(\omega_{d} t\right)\right]\right\}, \quad \text { Equation F-37 }\end{array}\right.$

where the initial conditions are again assumed zero: $\delta_{1}(0)=\dot{\delta}_{1}(0)=0$, and

$\widetilde{A_{1}}=A_{1}\left[\frac{1}{\sqrt{\left(1-r^{2}\right)^{2}+(2 \zeta r)^{2}}}\right]$

Equation F-38

$\widetilde{\varphi_{1}}=\tan ^{-1}\left(\frac{2 \zeta r}{1-r^{2}}\right)$

Equation F-39 
are the amplitude and relative phase of the forced response, respectively (Rao and Yap, 1995). The pulse or driving frequency is $\Omega$, and $r$ is the frequency ratio:

$\Omega=n_{c} \pi / \Delta t$

Equation F-40

$r=\Omega / \omega_{n}$

Equation F-41

For $\Delta t \leq t$, the response, $\delta=\delta_{2}(t)$, is again a free a free vibration, and has the same mathematical form as the solution to the square pulse:

$\delta_{2}(t)=\widetilde{A_{2}} e^{-\zeta \omega_{n}(t-\Delta t)} \cos \left(\omega_{d}(t-\Delta t)-\widetilde{\varphi_{2}}\right)$,

Equation F-42

where

$\widetilde{A_{2}}=\frac{\sqrt{\widetilde{\delta}_{\Delta t}{ }^{2} \omega_{n}{ }^{2}+\dot{\tilde{\delta}}_{\Delta t}{ }^{2}+2 \widetilde{\delta}_{\Delta t} \dot{\tilde{\delta}}_{\Delta t} \zeta \omega_{n}}}{\omega_{d}}$,

Equation F-43

$\widetilde{\varphi_{2}}=\tan ^{-1}\left(\frac{\dot{\tilde{\delta}}_{\Delta t}+\zeta \omega_{n} \widetilde{\delta}_{\Delta t}}{\widetilde{\delta}_{\Delta t} \omega_{d}}\right)$

Equation F-44

$\tilde{\delta}_{\Delta t}=\widetilde{A_{1}}\left[\begin{array}{c}\sin \left(n_{c} \pi-\widetilde{\varphi_{1}}\right)+\cdots \\ \left.e^{-\zeta \omega_{n} \Delta t}\left[\sin \widetilde{\varphi_{1}} \cos \left(\omega_{d} \Delta t\right)+\left(\frac{\zeta \omega_{n} \sin \widetilde{\varphi_{1}}-\Omega \cos \widetilde{\varphi_{1}}}{\omega_{d}}\right) \sin \left(\omega_{d} \Delta t\right)\right]\right]\end{array}\right.$

Equation F-45

and

$\dot{\tilde{\delta}}_{\Delta t}=\widetilde{A_{1}}\left\{\left[\begin{array}{c}\Omega \cos \left(n_{c} \pi-\widetilde{\varphi_{1}}\right)-\zeta \omega_{n} e^{-\zeta \omega_{n} \Delta t} \ldots \\ \sin \widetilde{\varphi_{1}} \cos \left(\omega_{d} \Delta t\right)+\left(\frac{\zeta \omega_{n} \sin \widetilde{\varphi_{1}}-\Omega \cos \widetilde{\varphi_{1}}}{\omega_{d}}\right) \sin \left(\omega_{d} \Delta t\right)+\frac{\sqrt{1-\zeta^{2}}}{\zeta} \ldots \\ {\left[\sin \widetilde{\varphi_{1}} \sin \left(\omega_{d} \Delta t\right)-\left(\zeta \omega_{n} \sin \widetilde{\varphi_{1}}-\Omega \cos \widetilde{\varphi_{1}}\right) \cos \left(\omega_{d} \Delta t\right)\right]}\end{array}\right]\right\}$.

Equation F-46

are the amplitude, phasing, and initial conditions of the free vibration response, respectively. The total response of the system can then be expressed as Equation F-19.

A closed-form solution to the maximum response of the system given a sinusoidal input can only be obtained when the peak response occurs during the free vibration $\left(t_{p}>\right.$ $\Delta t)$. If the peak occurs during a forced vibration $\left(t_{p}<\Delta t\right)$, the solution for the time to peak 
involves solving a transcendental equation, which can only be accomplished numerically. For a half cycle pulse, $t_{p}$ can be determined analytically for a subset of short duration pulses; however, for the full cycle pulse, $t_{p}<\Delta t$ for most $\Delta t ; t_{p}>\Delta t$ only for a window of durations (near resonance), where the maximum magnitude switches from occurring only in the positive response phase of the time history ( $1^{\text {st }}$ peak) to the negative response phase of the time history ( $2^{\text {nd }}$ peak). This switching results in an inflection in the contour plot surface, which becomes more apparent at lower damping ratios (Figure 4-4, top row). Thus a closed-form solution for the maximum response under sinusoidal excitation cannot be determined for a broad range of durations including system resonance.

In conclusion, the purpose of the derivations provided in this appendix are to mathematically derive the governing behaviors observed in a $2^{\text {nd }}$ order mechanical system for pulses of varying magnitude, duration, and shape. While the maximum magnitude of deformation response (contour plots) exhibits a sensitivity to the system parameters and pulse shape (Figures 4-4 and F-3), the general behaviors are consistent; deformations occurring due to shorter duration pulses are dependent primarily on velocity change, while deformations occurring due to longer duration pulses are dependent primarily on acceleration magnitude. The switch-over between the two regimes occurs when pulse durations approach the natural period of the system. Given that the application of the metrics developed in this dissertation involve arbitrary pulse shapes, and that the simplest pulse shape requires a switching function (Equations F-34 and F-35), provides justification for the current mathematical formulation of UBrIC. 


\section{Appendix G - Solution to the mDOF mechanical system}

The solutions for the system displacements of the MB model (Equation 6-1) were obtained using modal analysis. Modal analysis is a technique used to decouple the EOMs of MB systems by expressing the solution as a linear combination of the normal modes (Rao and Yap, 1995). This is accomplished by writing the EOM in terms of the natural mode shapes $[X]$ and principal coordinates $\vec{q}$ :

$[m][X] \ddot{\vec{q}}+[c][X] \dot{\vec{q}}+[k][X] \vec{q}=\vec{F}$, Equation G-1 where the system displacements can be expressed as $\vec{\delta}=[X] \vec{q}$

Equation G-2

Pre-multiplying Equation G-1 by the transpose of the modal matrix $[X]^{T}$, and assuming proportional damping, the following three, uncoupled, second order, ordinary differential equations are obtained:

$\ddot{q}_{j}+\left(a_{o}+a_{1} 4 \pi^{2} \widetilde{\Delta t}_{n_{j}}^{-2}\right) \dot{q}_{j}+4 \pi^{2} \widetilde{\Delta t}_{n_{j}}^{-2} q_{j}=Q_{j}$

Equation G-3

where $\widetilde{\Delta t}_{n_{j}}$ are the undamped natural periods for the $j^{\text {th }}$ normal mode $j=1,2,3$; and $Q_{j}$ are the generalized forces; $[X]^{T}[m] \ddot{\vec{u}}$. The modal damping ratios, $\tilde{\zeta}_{j}$, are determined using Equation 6-11 and $\widetilde{\Delta t}_{n_{j}}$ :

$\tilde{\zeta}_{j}=\frac{a_{1}+a_{o}\left(\frac{\widetilde{\Delta t}_{n_{j}}}{2 \pi}\right)^{2}}{\widetilde{\Delta t}_{n_{j}} / \pi}$.

Equation G-4

In this dissertation, the MB system was assumed to be underdamped $\left(0<\tilde{\zeta}_{i}<1\right)$. For a sinusoidal pulse shape, the solution to Equation $\mathrm{G}-3, q_{j}$, is the same as the sDOF system, and can be obtained analytically using Equation F-19. Finally, the physical displacements of the MB model, $\vec{\delta}(t)$ can be obtained using Equation G-2. 


\section{Appendix H - Supplemental Results from Chapter 5}

(a) Correlations with automotive and sled impact conditions

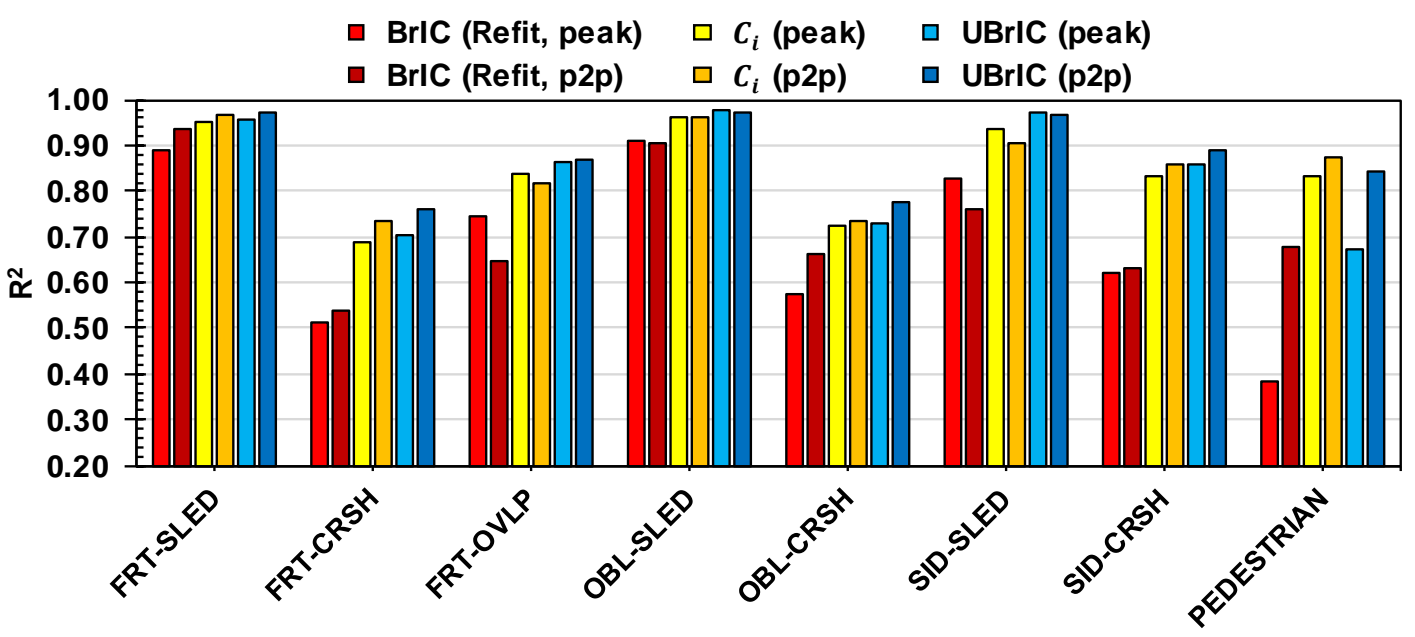

(b) Metric-accuracy with automotive and sled impact conditions

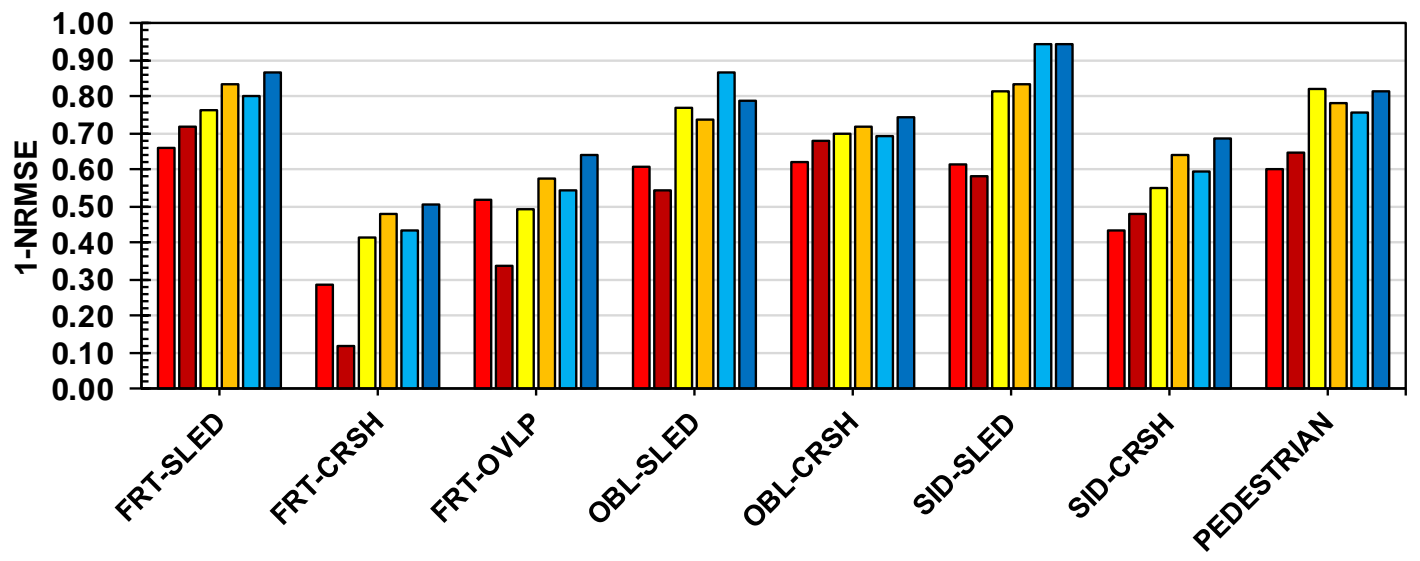

(c) Correlations with football impact conditions

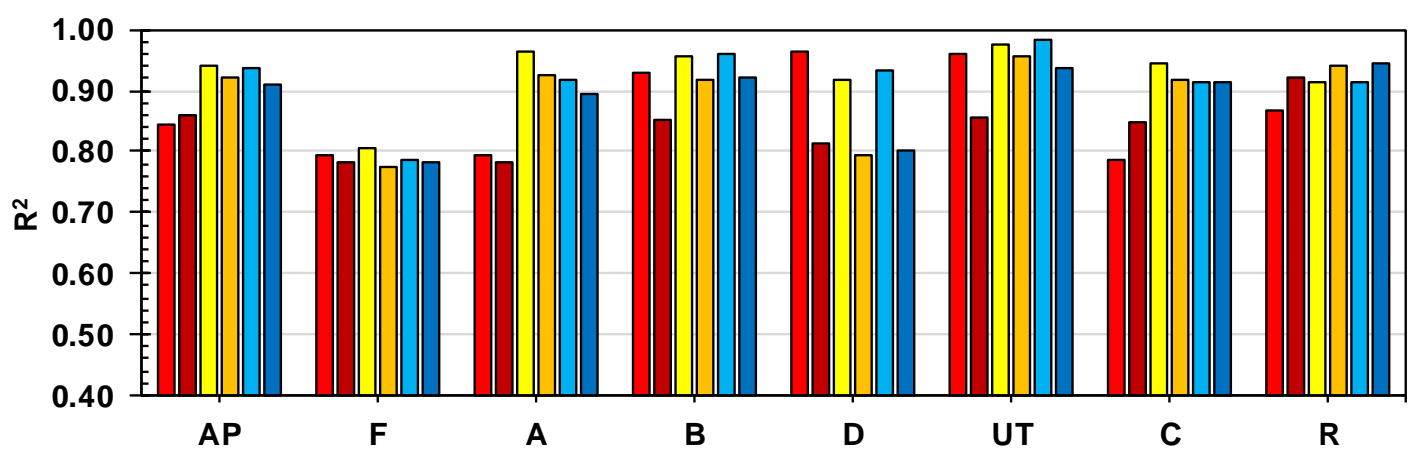


(d) Metric-accuracy with football impact conditions

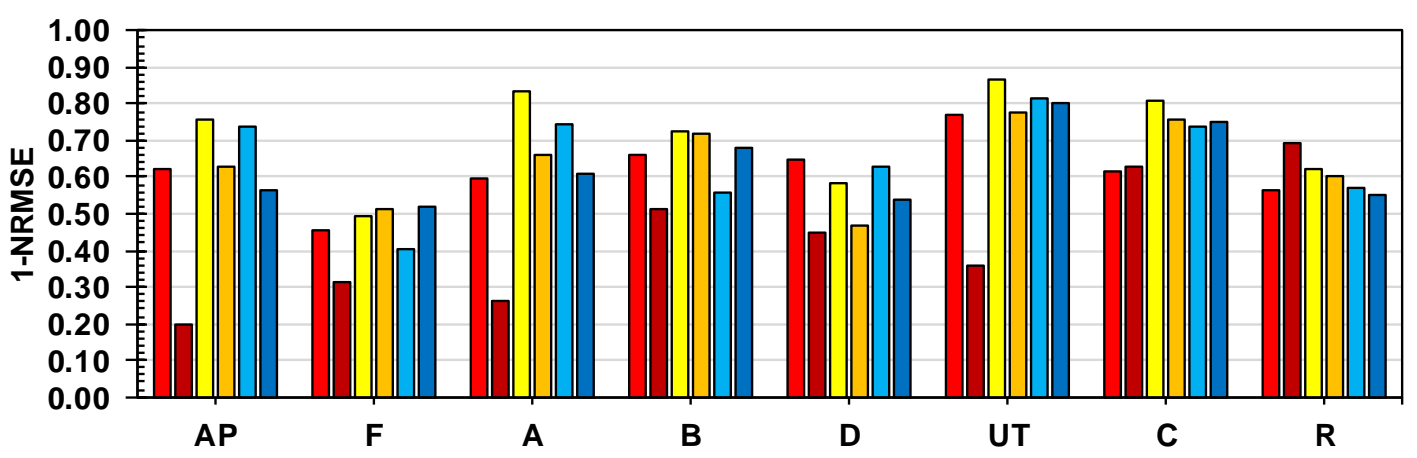

Figure H-1: Performance of fitted metrics assessed by impact condition based on correlations $(A, C)$ and metric accuracy $(B, D)$ for automotive and sled $(A, B)$, and football (C, D) impact conditions. Results are evaluated for metrics with $r=1,2$, and relative to MPS $\left(50^{\text {th }}\right.$ percentile) from GHBMC. Sample sizes for each impact condition are consistent with Figure 5-4.

(a) Correlations with automotive and sled impact conditions

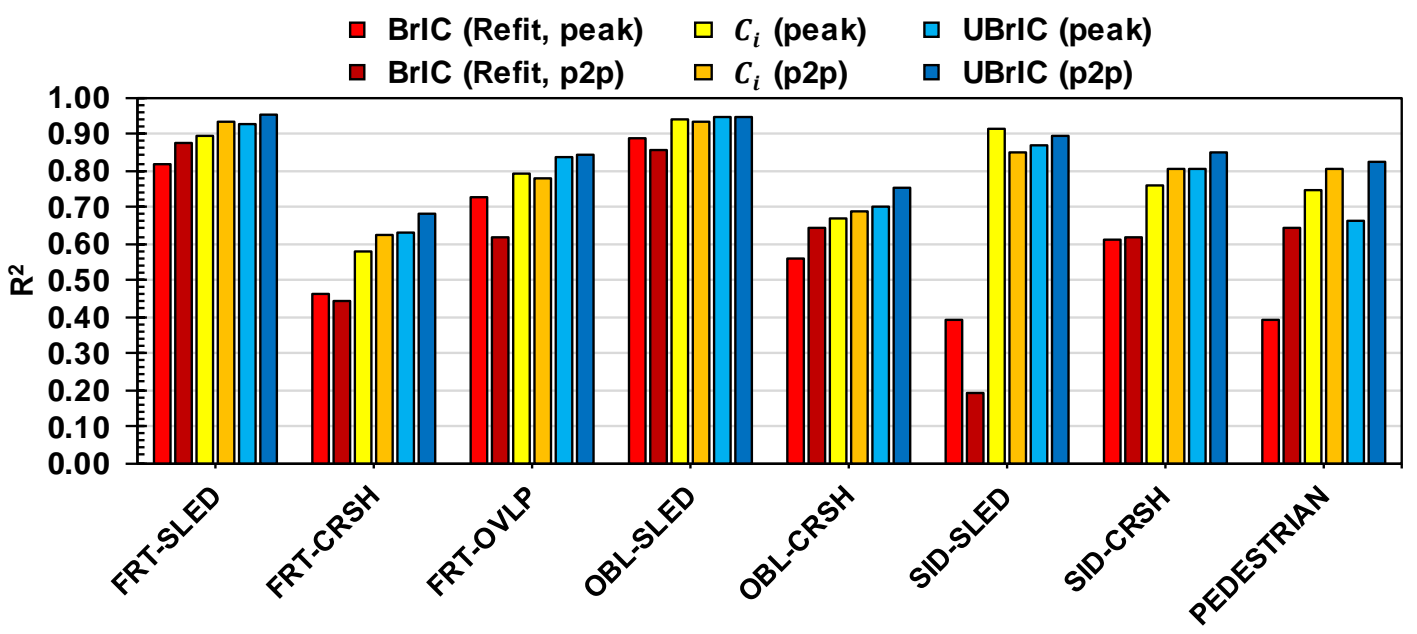

(b) Metric-accuracy with automotive and sled impact conditions

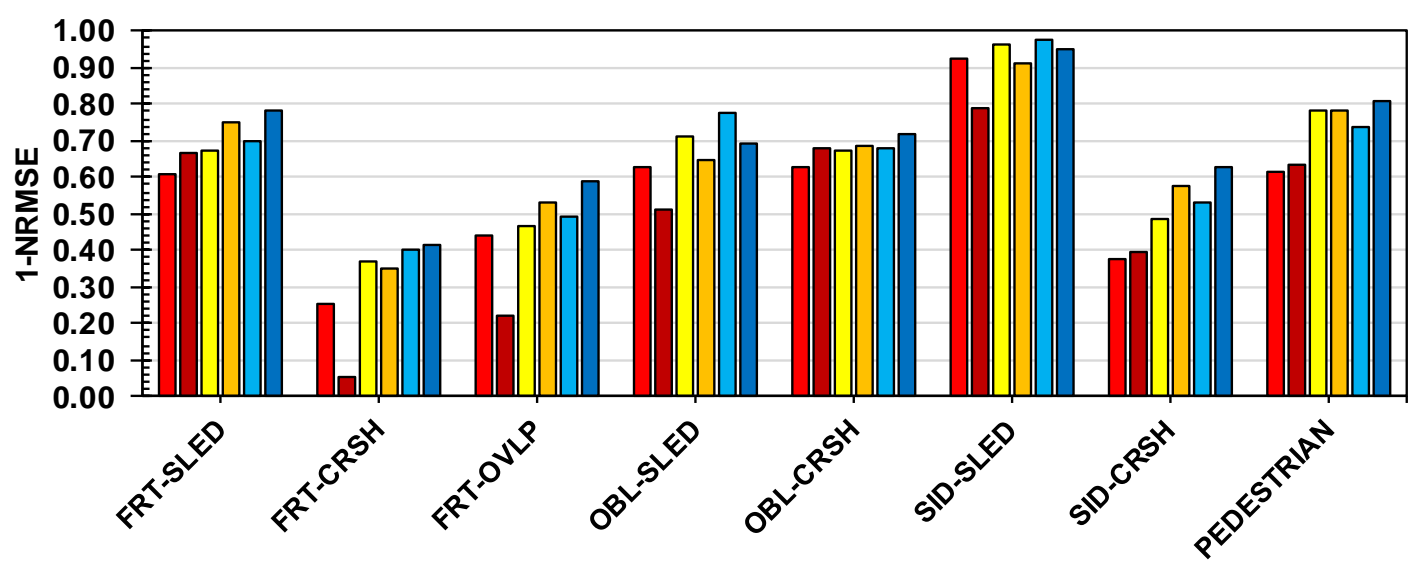


(c) Correlations with football impact conditions

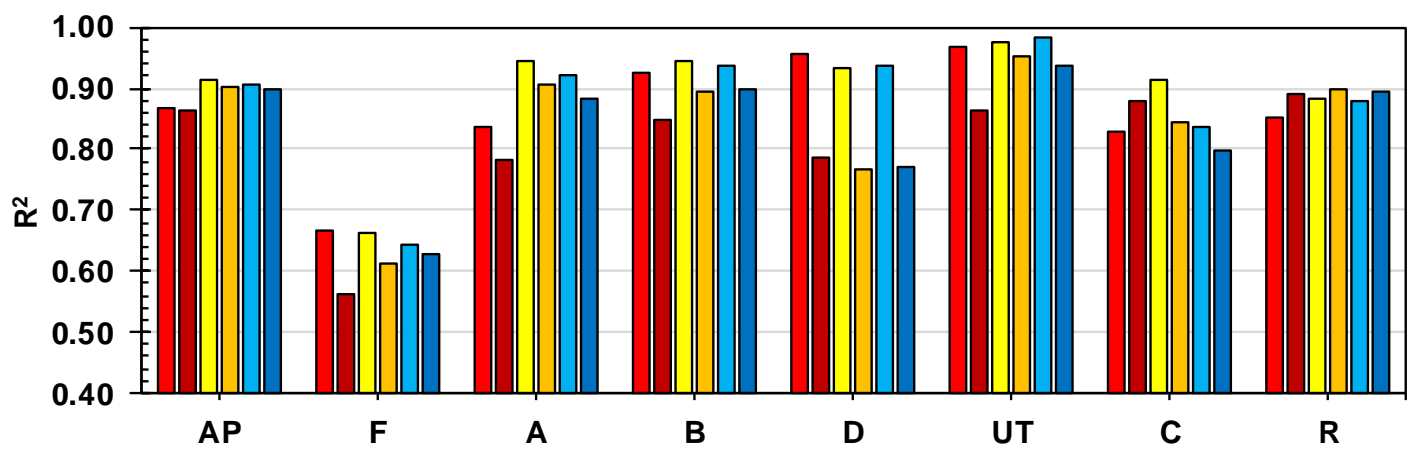

(d) Metric-accuracy with football impact conditions

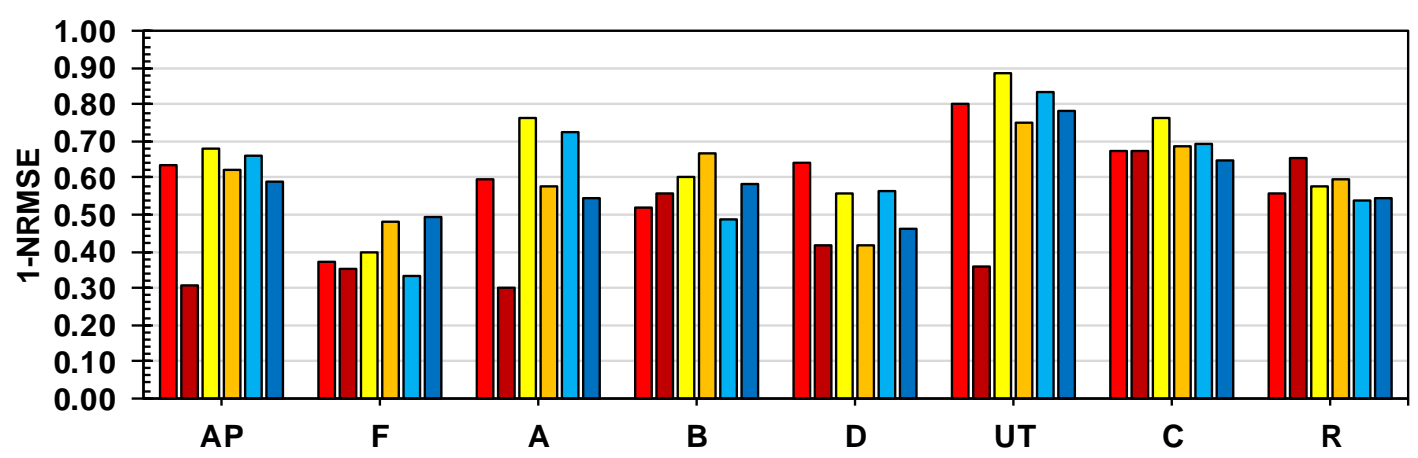

Figure H-2: Performance of fitted metrics assessed by impact condition based on correlations (A, C) and metric accuracy (B, D) for automotive and sled (A, B), and football (C, D) impact conditions. Results are evaluated for metrics with $r=1,2$, and relative to CSDM (25\% MPS threshold) from GHBMC. Sample sizes for each impact condition are consistent with Figure 5-4. 


\section{Appendix I - List of Head Impacts from the Database}

\section{I.1 UVa Sled Tests}

\begin{tabular}{|c|c|c|c|}
\hline $\begin{array}{c}\text { Source } \\
\text { Test \# }\end{array}$ & $\begin{array}{c}\text { Impact } \\
\text { Condition } \\
\end{array}$ & Test Details & Surrogate \\
\hline Sed D3 & PEDESTRIAN & VEHICLE SLED LATERALLY INTO PEDESTRIAN & POLAR II, M50 \\
\hline Sed P1 & PEDESTRIAN & VEHICLE SLED LATERALLY INTO PEDESTRIAN & PMHS, F \\
\hline Sed P2 & PEDESTRIAN & VEHICLE SLED LATERALLY INTO PEDESTRIAN & PMHS, M \\
\hline Sed P3 & PEDESTRIAN & VEHICLE SLED LATERALLY INTO PEDESTRIAN & PMHS, M \\
\hline SUV D1 & PEDESTRIAN & VEHICLE SLED LATERALLY INTO PEDESTRIAN & POLAR II, M50 \\
\hline SUV D2 & PEDESTRIAN & VEHICLE SLED LATERALLY INTO PEDESTRIAN & POLAR II, M50 \\
\hline SUV D3 & PEDESTRIAN & VEHICLE SLED LATERALLY INTO PEDESTRIAN & POLAR II, M50 \\
\hline SUV P1 & PEDESTRIAN & VEHICLE SLED LATERALLY INTO PEDESTRIAN & PMHS, F \\
\hline SUV P3 & PEDESTRIAN & VEHICLE SLED LATERALLY INTO PEDESTRIAN & PMHS, M \\
\hline Sed D1 & PEDESTRIAN & VEHICLE SLED LATERALLY INTO PEDESTRIAN & POLAR II, M50 \\
\hline Sed D2 & PEDESTRIAN & VEHICLE SLED LATERALLY INTO PEDESTRIAN & POLAR II, M50 \\
\hline Sed DA1 & PEDESTRIAN & VEHICLE SLED LATERALLY INTO PEDESTRIAN & POLAR II, M50 \\
\hline Sed DA2 & PEDESTRIAN & VEHICLE SLED LATERALLY INTO PEDESTRIAN & POLAR II, M50 \\
\hline SUV DA1 & PEDESTRIAN & VEHICLE SLED LATERALLY INTO PEDESTRIAN & POLAR II, M50 \\
\hline SUV DA2 & PEDESTRIAN & VEHICLE SLED LATERALLY INTO PEDESTRIAN & POLAR II, M50 \\
\hline S2 & PEDESTRIAN & VEHICLE SLED LATERALLY INTO PEDESTRIAN & PMHS, F \\
\hline $\mathrm{T} 7$ & PEDESTRIAN & VEHICLE SLED LATERALLY INTO PEDESTRIAN & PMHS, M \\
\hline S1 & PEDESTRIAN & VEHICLE SLED LATERALLY INTO PEDESTRIAN & PMHS, F \\
\hline S3 & PEDESTRIAN & VEHICLE SLED LATERALLY INTO PEDESTRIAN & PMHS, F \\
\hline T6 & PEDESTRIAN & VEHICLE SLED LATERALLY INTO PEDESTRIAN & PMHS, M \\
\hline M5 & PEDESTRIAN & VEHICLE SLED LATERALLY INTO PEDESTRIAN & PMHS, F \\
\hline M4 & PEDESTRIAN & VEHICLE SLED LATERALLY INTO PEDESTRIAN & PMHS, F \\
\hline 1134 & PEDESTRIAN & VEHICLE SLED LATERALLY INTO PEDESTRIAN & POLAR II, M50 \\
\hline 1136 & PEDESTRIAN & VEHICLE SLED LATERALLY INTO PEDESTRIAN & POLAR II, M50 \\
\hline 1137 & PEDESTRIAN & VEHICLE SLED LATERALLY INTO PEDESTRIAN & PMHS, 326, M \\
\hline 1138 & PEDESTRIAN & VEHICLE SLED LATERALLY INTO PEDESTRIAN & PMHS, 328, M \\
\hline 1140 & PEDESTRIAN & VEHICLE SLED LATERALLY INTO PEDESTRIAN & PMHS, 352, M \\
\hline 1141 & PEDESTRIAN & VEHICLE SLED LATERALLY INTO PEDESTRIAN & PMHS, 353, M \\
\hline S0077 & SIDE & $90^{\circ}$ FAR SIDE PURE LATERAL SLED & PMHS, 557, M \\
\hline S0078 & SIDE & $90^{\circ}$ FAR SIDE PURE LATERAL SLED & PMHS, 557, M \\
\hline S0079 & SIDE & $90^{\circ}$ FAR SIDE PURE LATERAL SLED & PMHS, 557, M \\
\hline S0080 & SIDE & $90^{\circ}$ FAR SIDE PURE LATERAL SLED & PMHS, 557, M \\
\hline S0082 & SIDE & $90^{\circ}$ FAR SIDE PURE LATERAL SLED & PMHS, 551, M \\
\hline S0083 & SIDE & $90^{\circ}$ FAR SIDE PURE LATERAL SLED & PMHS, 551, M \\
\hline S0084 & SIDE & $90^{\circ}$ FAR SIDE PURE LATERAL SLED & PMHS, 551, M \\
\hline S0085 & SIDE & $90^{\circ}$ FAR SIDE PURE LATERAL SLED & PMHS, 551, M \\
\hline S0086 & SIDE & $90^{\circ}$ FAR SIDE PURE LATERAL SLED & PMHS, 551, M \\
\hline S0087 & SIDE & $90^{\circ} \mathrm{FAR}$ SIDE PURE LATERAL SLED & PMHS, 559, M \\
\hline S0088 & SIDE & $90^{\circ}$ FAR SIDE PURE LATERAL SLED & PMHS, 559, M \\
\hline S0089 & SIDE & $90^{\circ}$ FAR SIDE PURE LATERAL SLED & PMHS, 559, M \\
\hline S0090 & SIDE & $90^{\circ}$ FAR SIDE PURE LATERAL SLED & PMHS, 559, M \\
\hline S0091 & SIDE & $90^{\circ}$ FAR SIDE PURE LATERAL SLED & PMHS, 559, M \\
\hline S0120 & OBLIQUE & $60^{\circ}$ FAR SIDE OBLIQUE SLED & PMHS, 591, M \\
\hline S0121 & OBLIQUE & $60^{\circ}$ FAR SIDE OBLIQUE SLED & PMHS, 591, M \\
\hline S0122 & OBLIQUE & $60^{\circ}$ FAR SIDE OBLIQUE SLED & PMHS, 591, M \\
\hline S0123 & OBLIQUE & $60^{\circ}$ FAR SIDE OBLIQUE SLED & PMHS, 591, M \\
\hline S0124 & OBLIQUE & $60^{\circ}$ FAR SIDE OBLIQUE SLED & PMHS, 591, M \\
\hline S0125 & OBLIQUE & $60^{\circ} \mathrm{FAR}$ SIDE OBLIQUE SLED & PMHS, 591, M \\
\hline S0126 & OBLIQUE & $60^{\circ}$ FAR SIDE OBLIQUE SLED & PMHS, 587, M \\
\hline S0127 & OBLIQUE & $60^{\circ} \mathrm{FAR}$ SIDE OBLIQUE SLED & PMHS, 587, M \\
\hline S0128 & OBLIQUE & $60^{\circ}$ FAR SIDE OBLIQUE SLED & PMHS, 587, M \\
\hline S0129 & OBLIQUE & $60^{\circ}$ FAR SIDE OBLIQUE SLED & PMHS, 587, M \\
\hline
\end{tabular}




\begin{tabular}{|c|c|c|c|}
\hline S0130 & OBLIQUE & $60^{\circ}$ FAR SIDE OBLIQUE SLED & PMHS, 587, M \\
\hline S0131 & OBLIQUE & $60^{\circ} \mathrm{FAR}$ SIDE OBLIQUE SLED & PMHS, 602, M \\
\hline S0132 & OBLIQUE & $60^{\circ}$ FAR SIDE OBLIQUE SLED & PMHS, 602, M \\
\hline S0133 & OBLIQUE & $60^{\circ}$ FAR SIDE OBLIQUE SLED & PMHS, 602, M \\
\hline S0134 & OBLIQUE & $60^{\circ}$ FAR SIDE OBLIQUE SLED & PMHS, 602, M \\
\hline S0135 & OBLIQUE & $60^{\circ} \mathrm{FAR}$ SIDE OBLIQUE SLED & PMHS, 602, M \\
\hline S0136 & OBLIQUE & $60^{\circ}$ FAR SIDE OBLIQUE SLED & PMHS, 608, M \\
\hline S0137 & OBLIQUE & $60^{\circ} \mathrm{FAR}$ SIDE OBLIQUE SLED & PMHS, 608, M \\
\hline S0138 & OBLIQUE & $60^{\circ} \mathrm{FAR}$ SIDE OBLIQUE SLED & PMHS, $608, \mathrm{M}$ \\
\hline S0139 & OBLIQUE & $60^{\circ}$ FAR SIDE OBLIQUE SLED & PMHS, 608, M \\
\hline S0140 & OBLIQUE & $60^{\circ}$ FAR SIDE OBLIQUE SLED & PMHS, 608, M \\
\hline S0238 & OBLIQUE & $20^{\circ} \mathrm{FAR}$ SIDE OBLIQUE SLED & H-III, M50 \\
\hline S0239 & OBLIQUE & $20^{\circ} \mathrm{FAR}$ SIDE OBLIQUE SLED & H-III, M50 \\
\hline S0243 & OBLIQUE & $20^{\circ} \mathrm{FAR}$ SIDE OBLIQUE SLED & PMHS, 642, M \\
\hline S0244 & OBLIQUE & $20^{\circ}$ FAR SIDE OBLIQUE SLED & PMHS, 605, M \\
\hline S0245 & OBLIQUE & $20^{\circ} \mathrm{FAR}$ SIDE OBLIQUE SLED & PMHS, $659, \mathrm{M}$ \\
\hline S0246 & OBLIQUE & $20^{\circ}$ FAR SIDE OBLIQUE SLED & H-III, M50 \\
\hline S0248 & OBLIQUE & $20^{\circ} \mathrm{FAR}$ SIDE OBLIQUE SLED & THOR, M50 \\
\hline S0249 & OBLIQUE & $20^{\circ}$ FAR SIDE OBLIQUE SLED & THOR, M50 \\
\hline S0250 & OBLIQUE & $20^{\circ} \mathrm{FAR}$ SIDE OBLIQUE SLED & THOR, M50 \\
\hline S0251 & OBLIQUE & $20^{\circ} \mathrm{FAR}$ SIDE OBLIQUE SLED & THOR, M50 \\
\hline S0156 & FRONTAL & UNIVERSAL / GOLD STANDARD 1 & THOR, M50 \\
\hline S0157 & FRONTAL & UNIVERSAL / GOLD STANDARD 1 & THOR, M50 \\
\hline S0158 & FRONTAL & UNIVERSAL / GOLD STANDARD 1 & THOR, M50 \\
\hline S0159 & FRONTAL & UNIVERSAL / GOLD STANDARD 2 & THOR, M50 \\
\hline S0160 & FRONTAL & UNIVERSAL / GOLD STANDARD 2 & THOR, M50 \\
\hline S0161 & FRONTAL & UNIVERSAL / GOLD STANDARD 2 & THOR, M50 \\
\hline S0163 & FRONTAL & UNIVERSAL / GOLD STANDARD DRIVER SIDE & THOR, M50 \\
\hline S0164 & FRONTAL & UNIVERSAL / GOLD STANDARD DRIVER SIDE & THOR, M50 \\
\hline S0166 & FRONTAL & UNIVERSAL / GOLD STANDARD DRIVER SIDE & THOR, M50 \\
\hline S0167 & FRONTAL & UNIVERSAL / GOLD STANDARD DRIVER SIDE & THOR, M50 \\
\hline S0169 & FRONTAL & UNIVERSAL / GOLD STANDARD DRIVER SIDE & H-III, M50 \\
\hline S0170 & FRONTAL & UNIVERSAL / GOLD STANDARD DRIVER SIDE & H-III, M50 \\
\hline S0171 & FRONTAL & UNIVERSAL / GOLD STANDARD 1 & H-III, M50 \\
\hline 1210 & FRONTAL & REAR SEAT FRONTAL SLED TEST & H-III, M50 \\
\hline 1211 & FRONTAL & REAR SEAT FRONTAL SLED TEST & H-III, M50 \\
\hline 1212 & FRONTAL & REAR SEAT FRONTAL SLED TEST & H-III, M50 \\
\hline 1215 & FRONTAL & REAR SEAT FRONTAL SLED TEST & H-III, M50 \\
\hline 1216 & FRONTAL & REAR SEAT FRONTAL SLED TEST & H-III, M50 \\
\hline 1217 & FRONTAL & REAR SEAT FRONTAL SLED TEST & H-III, M50 \\
\hline 1218 & FRONTAL & REAR SEAT FRONTAL SLED TEST & H-III, F05 \\
\hline 1219 & FRONTAL & REAR SEAT FRONTAL SLED TEST & H-III, F05 \\
\hline 1220 & FRONTAL & REAR SEAT FRONTAL SLED TEST & H-III, F05 \\
\hline 1221 & FRONTAL & REAR SEAT FRONTAL SLED TEST & H-III, F05 \\
\hline 1222 & FRONTAL & REAR SEAT FRONTAL SLED TEST & H-III, F05 \\
\hline 1223 & FRONTAL & REAR SEAT FRONTAL SLED TEST & H-III, F05 \\
\hline 1247 & FRONTAL & REAR SEAT FRONTAL SLED TEST & THOR, M50 \\
\hline 1248 & FRONTAL & REAR SEAT FRONTAL SLED TEST & THOR, M50 \\
\hline 1249 & FRONTAL & REAR SEAT FRONTAL SLED TEST & THOR, M50 \\
\hline 1250 & FRONTAL & REAR SEAT FRONTAL SLED TEST & THOR, M50 \\
\hline 1251 & FRONTAL & REAR SEAT FRONTAL SLED TEST & THOR, M50 \\
\hline 1252 & FRONTAL & REAR SEAT FRONTAL SLED TEST & THOR, M50 \\
\hline 1256 & FRONTAL & REAR SEAT FRONTAL SLED TEST & THOR, M50 \\
\hline 1257 & FRONTAL & REAR SEAT FRONTAL SLED TEST & THOR, M50 \\
\hline 1258 & FRONTAL & REAR SEAT FRONTAL SLED TEST & THOR, M50 \\
\hline 1259 & FRONTAL & REAR SEAT FRONTAL SLED TEST & THOR, M50 \\
\hline 1260 & FRONTAL & REAR SEAT FRONTAL SLED TEST & THOR, M50 \\
\hline 1261 & FRONTAL & REAR SEAT FRONTAL SLED TEST & THOR, M50 \\
\hline
\end{tabular}




\section{I.2 NHTSA Crash Tests}

\begin{tabular}{|c|c|c|c|c|}
\hline $\begin{array}{l}\text { Source } \\
\text { Test \# }\end{array}$ & $\begin{array}{l}\text { Occ. } \\
\text { Loc. }\end{array}$ & $\begin{array}{c}\text { Impact } \\
\text { Condition }\end{array}$ & Test Details & Surrogate \\
\hline 4303 & 01 & FRONTAL & VEHICLE INTO BARRIER $(0,0)$ & H-III, M50 \\
\hline 4242 & 01 & FRONTAL & VEHICLE INTO BARRIER $(0,0)$ & H-III, M50 \\
\hline 4205 & 02 & FRONTAL & VEHICLE INTO BARRIER $(0,0)$ & H-III, M50 \\
\hline 4273 & 01 & FRONTAL & VEHICLE INTO BARRIER $(0,0)$ & H-III, M50 \\
\hline 4198 & 01 & FRONTAL & VEHICLE INTO BARRIER $(0,0)$ & H-III, M50 \\
\hline 3897 & 01 & FRONTAL & VEHICLE INTO BARRIER $(0,0)$ & H-III, M50 \\
\hline 4266 & 02 & FRONTAL & VEHICLE INTO BARRIER $(0,0)$ & H-III, M50 \\
\hline 4247 & 01 & FRONTAL & VEHICLE INTO BARRIER $(0,0)$ & H-III, M50 \\
\hline 3916 & 01 & FRONTAL & VEHICLE INTO BARRIER $(0,0)$ & H-III, M50 \\
\hline 4264 & 02 & FRONTAL & VEHICLE INTO BARRIER $(0,0)$ & H-III, M50 \\
\hline 3901 & 02 & FRONTAL & VEHICLE INTO BARRIER $(0,0)$ & H-III, M50 \\
\hline 4250 & 01 & FRONTAL & VEHICLE INTO BARRIER $(0,0)$ & H-III, M50 \\
\hline 4251 & 01 & FRONTAL & VEHICLE INTO BARRIER $(0,0)$ & H-III, M50 \\
\hline 4215 & 02 & FRONTAL & VEHICLE INTO BARRIER $(0,0)$ & H-III, M50 \\
\hline 4237 & 02 & FRONTAL & VEHICLE INTO BARRIER $(0,0)$ & H-III, M50 \\
\hline 4080 & 01 & FRONTAL & VEHICLE INTO BARRIER $(0,0)$ & H-III, M50 \\
\hline 4090 & 01 & FRONTAL & VEHICLE INTO BARRIER $(0,0)$ & H-III, M50 \\
\hline 4264 & 01 & FRONTAL & VEHICLE INTO BARRIER $(0,0)$ & H-III, M50 \\
\hline 4090 & 02 & FRONTAL & VEHICLE INTO BARRIER $(0,0)$ & H-III, M50 \\
\hline 4223 & 02 & FRONTAL & VEHICLE INTO BARRIER $(0,0)$ & H-III, M50 \\
\hline 4267 & 02 & FRONTAL & VEHICLE INTO BARRIER $(0,0)$ & H-III, M50 \\
\hline 4215 & 01 & FRONTAL & VEHICLE INTO BARRIER $(0,0)$ & H-III, M50 \\
\hline 4242 & 02 & FRONTAL & VEHICLE INTO BARRIER $(0,0)$ & H-III, M50 \\
\hline 4259 & 01 & FRONTAL & VEHICLE INTO BARRIER $(0,0)$ & H-III, M50 \\
\hline 3987 & 01 & FRONTAL & VEHICLE INTO BARRIER $(0,0)$ & H-III, M50 \\
\hline 4255 & 02 & FRONTAL & VEHICLE INTO BARRIER $(0,0)$ & H-III, M50 \\
\hline 4235 & 01 & FRONTAL & VEHICLE INTO BARRIER $(0,0)$ & H-III, M50 \\
\hline 4235 & 02 & FRONTAL & VEHICLE INTO BARRIER $(0,0)$ & H-III, M50 \\
\hline 4265 & 02 & FRONTAL & VEHICLE INTO BARRIER $(0,0)$ & H-III, M50 \\
\hline 4249 & 02 & FRONTAL & VEHICLE INTO BARRIER $(0,0)$ & H-III, M50 \\
\hline 4240 & 01 & FRONTAL & VEHICLE INTO BARRIER $(0,0)$ & H-III, M50 \\
\hline 4237 & 01 & FRONTAL & VEHICLE INTO BARRIER $(0,0)$ & H-III, M50 \\
\hline 4259 & 02 & FRONTAL & VEHICLE INTO BARRIER $(0,0)$ & H-III, M50 \\
\hline 4198 & 02 & FRONTAL & VEHICLE INTO BARRIER $(0,0)$ & H-III, M50 \\
\hline 3915 & 02 & FRONTAL & VEHICLE INTO BARRIER $(0,0)$ & H-III, M50 \\
\hline 3901 & 01 & FRONTAL & VEHICLE INTO BARRIER $(0,0)$ & H-III, M50 \\
\hline 4241 & 01 & FRONTAL & VEHICLE INTO BARRIER $(0,0)$ & H-III, M50 \\
\hline 4252 & 01 & FRONTAL & VEHICLE INTO BARRIER $(0,0)$ & H-III, M50 \\
\hline 3952 & 02 & FRONTAL & VEHICLE INTO BARRIER $(0,0)$ & H-III, M50 \\
\hline 5287 & 01 & FRONTAL & VEHICLE INTO BARRIER $(0,0)$ & H-III, M50 \\
\hline 5301 & 02 & FRONTAL & VEHICLE INTO BARRIER $(0,0)$ & H-III, M50 \\
\hline 5567 & 01 & FRONTAL & VEHICLE INTO BARRIER $(0,0)$ & H-III, M50 \\
\hline 5594 & 02 & FRONTAL & VEHICLE INTO BARRIER $(0,0)$ & H-III, M50 \\
\hline 5595 & 01 & FRONTAL & VEHICLE INTO BARRIER $(0,0)$ & H-III, M50 \\
\hline 5595 & 02 & FRONTAL & VEHICLE INTO BARRIER $(0,0)$ & H-III, M50 \\
\hline 5609 & 01 & FRONTAL & VEHICLE INTO BARRIER $(0,0)$ & H-III, M50 \\
\hline 5613 & 01 & FRONTAL & VEHICLE INTO BARRIER $(0,0)$ & H-III, M50 \\
\hline 5711 & 01 & FRONTAL & VEHICLE INTO BARRIER $(0,0)$ & H-III, M50 \\
\hline 5715 & 01 & FRONTAL & VEHICLE INTO BARRIER $(0,0)$ & H-III, M50 \\
\hline 6370 & 01 & FRONTAL & VEHICLE INTO BARRIER $(0,50)$ & H-III, M50 \\
\hline 7966 & 01 & FRONTAL & VEHICLE INTO BARRIER $(0,0)$ & H-III, M50 \\
\hline 7977 & 01 & FRONTAL & VEHICLE INTO BARRIER $(0,0)$ & H-III, M50 \\
\hline 7978 & 01 & FRONTAL & VEHICLE INTO BARRIER $(0,0)$ & H-III, M50 \\
\hline 7989 & 01 & FRONTAL & VEHICLE INTO BARRIER $(0,0)$ & H-III, M50 \\
\hline 8000 & 01 & FRONTAL & VEHICLE INTO BARRIER $(0,0)$ & H-III, M50 \\
\hline 8024 & 01 & FRONTAL & VEHICLE INTO BARRIER $(0,0)$ & H-III, M50 \\
\hline 8035 & 01 & FRONTAL & VEHICLE INTO BARRIER $(0,0)$ & H-III, M50 \\
\hline
\end{tabular}




\begin{tabular}{|c|c|c|c|c|}
\hline 8045 & 01 & FRONTAL & VEHICLE INTO BARRIER $(0,0)$ & H-III, M50 \\
\hline 8048 & 01 & FRONTAL & VEHICLE INTO BARRIER $(0,0)$ & H-III, M50 \\
\hline 8055 & 01 & FRONTAL & VEHICLE INTO BARRIER $(0,0)$ & H-III, M50 \\
\hline 8064 & 01 & FRONTAL & VEHICLE INTO BARRIER $(0,0)$ & H-III, M50 \\
\hline 8068 & 01 & FRONTAL & VEHICLE INTO BARRIER $(0,0)$ & H-III, M50 \\
\hline 8071 & 01 & FRONTAL & VEHICLE INTO BARRIER $(0,0)$ & H-III, M50 \\
\hline 8077 & 01 & FRONTAL & VEHICLE INTO BARRIER $(0,0)$ & H-III, M50 \\
\hline 8081 & 01 & FRONTAL & VEHICLE INTO BARRIER $(0,0)$ & H-III, M50 \\
\hline 8091 & 01 & FRONTAL & VEHICLE INTO BARRIER $(0,0)$ & H-III, M50 \\
\hline 8106 & 01 & FRONTAL & VEHICLE INTO BARRIER $(0,0)$ & H-III, M50 \\
\hline 8153 & 01 & FRONTAL & VEHICLE INTO BARRIER $(0,0)$ & H-III, M50 \\
\hline 8151 & 01 & FRONTAL & VEHICLE INTO BARRIER $(0,0)$ & H-III, M50 \\
\hline 8156 & 01 & FRONTAL & VEHICLE INTO BARRIER $(0,0)$ & H-III, M50 \\
\hline 6830 & 01 & OBLIQUE & VEHICLE INTO VEHICLE $(15,50)$ & THOR, M50 \\
\hline 6831 & 01 & OBLIQUE & VEHICLE INTO VEHICLE $(15,50)$ & THOR, M50 \\
\hline 6852 & 01 & OBLIQUE & IMPACTOR INTO VEHICLE $(15,50)$ & THOR, M50 \\
\hline 6855 & 01 & OBLIQUE & IMPACTOR INTO VEHICLE $(15,18)$ & THOR, M50 \\
\hline 6872 & 01 & OBLIQUE & VEHICLE INTO IMPACTOR $(4,26)$ & THOR, M50 \\
\hline 6873 & 01 & OBLIQUE & VEHICLE INTO IMPACTOR $(6,26)$ & THOR, M50 \\
\hline 6937 & 01 & OBLIQUE & IMPACTOR INTO VEHICLE $(15,50)$ & THOR, M50 \\
\hline 7144 & 01 & OBLIQUE & IMPACTOR INTO VEHICLE $(9,50)$ & THOR, M50 \\
\hline 7145 & 01 & OBLIQUE & VEHICLE INTO POLE $(7,10)$ & THOR, M50 \\
\hline 7292 & 01 & OBLIQUE & VEHICLE INTO VEHICLE (7) & THOR, M50 \\
\hline 7293 & 01 & OBLIQUE & VEHICLE INTO VEHICLE (7) & THOR, M50 \\
\hline 7366 & 01 & OBLIQUE & IMPACTOR INTO VEHICLE $(15,35)$ & THOR, M50 \\
\hline 7368 & 01 & OBLIQUE & IMPACTOR INTO VEHICLE $(7,20)$ & THOR, M50 \\
\hline 7371 & 01 & OBLIQUE & VEHICLE INTO VEHICLE $(15,50)$ & THOR, M50 \\
\hline 7429 & 01 & OBLIQUE & IMPACTOR INTO VEHICLE $(15,35)$ & THOR, M50 \\
\hline 7433 & 01 & OBLIQUE & IMPACTOR INTO VEHICLE $(15,35)$ & THOR, M50 \\
\hline 7434 & 01 & OBLIQUE & IMPACTOR INTO VEHICLE $(7,20)$ & THOR, M50 \\
\hline 7428 & 01 & OBLIQUE & IMPACTOR INTO VEHICLE $(15,35)$ & THOR, M50 \\
\hline 7431 & 01 & OBLIQUE & IMPACTOR INTO VEHICLE $(15,35)$ & THOR, M50 \\
\hline 7427 & 01 & OBLIQUE & IMPACTOR INTO VEHICLE $(7,20)$ & THOR, M50 \\
\hline 7432 & 01 & OBLIQUE & IMPACTOR INTO VEHICLE $(7,20)$ & THOR, M50 \\
\hline 7430 & 01 & OBLIQUE & IMPACTOR INTO VEHICLE $(7,20)$ & THOR, M50 \\
\hline 7426 & 01 & OBLIQUE & IMPACTOR INTO VEHICLE $(7,20)$ & THOR, M50 \\
\hline 7458 & 01 & OBLIQUE & IMPACTOR INTO VEHICLE $(15,35)$ & THOR, M50 \\
\hline 7441 & 01 & OBLIQUE & IMPACTOR INTO VEHICLE $(15,35)$ & THOR, M50 \\
\hline 7457 & 01 & OBLIQUE & IMPACTOR INTO VEHICLE $(15,35)$ & THOR, M50 \\
\hline 7444 & 01 & OBLIQUE & IMPACTOR INTO VEHICLE $(7,20)$ & THOR, M50 \\
\hline $7456 \dagger$ & 01 & OBLIQUE & IMPACTOR INTO VEHICLE $(7,20)$ & THOR, M50 \\
\hline 7467 & 01 & OBLIQUE & IMPACTOR INTO VEHICLE $(15,35)$ & THOR, M50 \\
\hline 7468 & 01 & OBLIQUE & IMPACTOR INTO VEHICLE $(7,20)$ & THOR, M50 \\
\hline 7476 & 01 & OBLIQUE & IMPACTOR INTO VEHICLE $(15,35)$ & THOR, M50 \\
\hline 7967 & 01 & SIDE & IMPACTOR INTO VEHICLE $(90,0)$ & ES-2RE, M50 \\
\hline 7984 & 01 & SIDE & IMPACTOR INTO VEHICLE $(90,0)$ & ES-2RE, M50 \\
\hline 7990 & 01 & SIDE & IMPACTOR INTO VEHICLE $(90,0)$ & ES-2RE, M50 \\
\hline 7998 & 01 & SIDE & IMPACTOR INTO VEHICLE $(90,0)$ & ES-2RE, M50 \\
\hline 8033 & 01 & SIDE & IMPACTOR INTO VEHICLE $(90,0)$ & ES-2RE, M50 \\
\hline 8047 & 01 & SIDE & IMPACTOR INTO VEHICLE $(90,0)$ & ES-2RE, M50 \\
\hline 8053 & 01 & SIDE & IMPACTOR INTO VEHICLE $(90,0)$ & ES-2RE, M50 \\
\hline 8054 & 01 & SIDE & IMPACTOR INTO VEHICLE $(90,0)$ & ES-2RE, M50 \\
\hline 8069 & 01 & SIDE & IMPACTOR INTO VEHICLE $(90,0)$ & ES-2RE, M50 \\
\hline 8072 & 01 & SIDE & IMPACTOR INTO VEHICLE $(90,0)$ & ES-2RE, M50 \\
\hline 8078 & 01 & SIDE & IMPACTOR INTO VEHICLE $(90,0)$ & ES-2RE, M50 \\
\hline 8079 & 01 & SIDE & IMPACTOR INTO VEHICLE $(90,0)$ & ES-2RE, M50 \\
\hline 8082 & 01 & SIDE & IMPACTOR INTO VEHICLE $(90,0)$ & ES-2RE, M50 \\
\hline 8092 & 01 & SIDE & IMPACTOR INTO VEHICLE $(90,0)$ & ES-2RE, M50 \\
\hline 8102 & 01 & SIDE & IMPACTOR INTO VEHICLE $(90,0)$ & ES-2RE, M50 \\
\hline 8108 & 01 & SIDE & IMPACTOR INTO VEHICLE $(90,0)$ & ES-2RE, M50 \\
\hline 8149 & 01 & SIDE & IMPACTOR INTO VEHICLE $(90,0)$ & ES-2RE, M50 \\
\hline 8157 & 01 & SIDE & IMPACTOR INTO VEHICLE $(90,0)$ & ES-2RE, M50 \\
\hline
\end{tabular}




\begin{tabular}{|c|c|c|c|c|}
\hline 8150 & 01 & SIDE & IMPACTOR INTO VEHICLE $(90,0)$ & ES-2RE, M50 \\
\hline 3800 & 01 & SIDE & IMPACTOR INTO VEHICLE $(90,0)$ & ES-2RE, M50 \\
\hline 4551 & 01 & SIDE & IMPACTOR INTO VEHICLE $(90,0)$ & ES-2RE, M50 \\
\hline 3875 & 01 & SIDE & IMPACTOR INTO VEHICLE $(90,0)$ & ES-2RE, M50 \\
\hline 3899 & 01 & SIDE & IMPACTOR INTO VEHICLE $(90,0)$ & ES-2RE, M50 \\
\hline 3818 & 01 & SIDE & VEHICLE INTO BARRIER $(90,0)$ & ES-2RE, M50 \\
\hline 4547 & 01 & SIDE & IMPACTOR INTO VEHICLE $(90,0)$ & ES-2RE, M50 \\
\hline 4380 & 01 & SIDE & IMPACTOR INTO VEHICLE $(90,0)$ & ES-2RE, M50 \\
\hline 3845 & 01 & SIDE & VEHICLE INTO BARRIER $(90,0)$ & ES-2RE, M50 \\
\hline 4497 & 01 & SIDE & VEHICLE INTO BARRIER $(75,0)$ & ES-2RE, M50 \\
\hline 4547 & 04 & SIDE & IMPACTOR INTO VEHICLE $(90,0)$ & ES-2RE, M50 \\
\hline 3898 & 01 & SIDE & VEHICLE INTO BARRIER $(90,0)$ & ES-2RE, M50 \\
\hline 4551 & 04 & SIDE & IMPACTOR INTO VEHICLE $(90,0)$ & ES-2RE, M50 \\
\hline 3799 & 04 & SIDE & IMPACTOR INTO VEHICLE $(90,0)$ & ES-2RE, M50 \\
\hline 4380 & 04 & SIDE & IMPACTOR INTO VEHICLE $(90,0)$ & ES-2RE, M50 \\
\hline 3820 & 01 & SIDE & VEHICLE INTO BARRIER $(90,0)$ & ES-2RE, M50 \\
\hline 4456 & 04 & SIDE & IMPACTOR INTO VEHICLE $(90,0)$ & ES-2RE, M50 \\
\hline 3819 & 04 & SIDE & IMPACTOR INTO VEHICLE $(90,0)$ & ES-2RE, M50 \\
\hline 4456 & 01 & SIDE & IMPACTOR INTO VEHICLE $(90,0)$ & ES-2RE, M50 \\
\hline 3799 & 01 & SIDE & IMPACTOR INTO VEHICLE $(90,0)$ & ES-2RE, M50 \\
\hline 4378 & 01 & SIDE & VEHICLE INTO BARRIER $(75,0)$ & ES-2RE, M50 \\
\hline 4292 & 01 & SIDE & VEHICLE INTO VEHICLE $(90,0)$ & ES-2RE, M50 \\
\hline 4498 & 01 & SIDE & VEHICLE INTO BARRIER $(75,0)$ & ES-2RE, M50 \\
\hline 3803 & 04 & SIDE & IMPACTOR INTO VEHICLE $(90,0)$ & ES-2RE, M50 \\
\hline 3802 & 01 & SIDE & VEHICLE INTO BARRIER $(90,0)$ & ES-2RE, M50 \\
\hline 4292 & 04 & SIDE & VEHICLE INTO VEHICLE $(90,0)$ & ES-2RE, M50 \\
\hline 3803 & 01 & SIDE & IMPACTOR INTO VEHICLE $(90,0)$ & ES-2RE, M50 \\
\hline 4482 & 01 & SIDE & VEHICLE INTO VEHICLE $(90,0)$ & ES-2RE, M50 \\
\hline 3800 & 04 & SIDE & IMPACTOR INTO VEHICLE $(90,0)$ & ES-2RE, M50 \\
\hline 4471 & 01 & SIDE & VEHICLE INTO BARRIER $(75,0)$ & ES-2RE, M50 \\
\hline 4313 & 01 & SIDE & VEHICLE INTO BARRIER $(75,0)$ & ES-2RE, M50 \\
\hline 3819 & 01 & SIDE & IMPACTOR INTO VEHICLE $(90,0)$ & ES-2RE, M50 \\
\hline 4859 & 01 & SIDE & VEHICLE INTO BARRIER $(75,0)$ & ES-2RE, M50 \\
\hline 5296 & 01 & SIDE & VEHICLE INTO BARRIER $(75,0)$ & ES-2RE, M50 \\
\hline 5317 & 01 & SIDE & VEHICLE INTO BARRIER $(75,0)$ & ES-2RE, M50 \\
\hline 5406 & 01 & SIDE & VEHICLE INTO BARRIER $(75,0)$ & ES-2RE, M50 \\
\hline 5407 & 01 & SIDE & VEHICLE INTO BARRIER $(75,0)$ & ES-2RE, M50 \\
\hline 5408 & 01 & SIDE & VEHICLE INTO BARRIER $(75,0)$ & ES-2RE, M50 \\
\hline 5416 & 01 & SIDE & VEHICLE INTO BARRIER $(75,0)$ & ES-2RE, M50 \\
\hline 5461 & 01 & SIDE & IMPACTOR INTO VEHICLE $(90,0)$ & ES-2RE, M50 \\
\hline 5472 & 01 & SIDE & VEHICLE INTO BARRIER $(75,0)$ & ES-2RE, M50 \\
\hline *7977 & 02 & FRONTAL & VEHICLE INTO BARRIER $(0,0)$ & H-III, F05 \\
\hline *7989 & 02 & FRONTAL & VEHICLE INTO BARRIER $(0,0)$ & H-III, F05 \\
\hline$* 8024$ & 02 & FRONTAL & VEHICLE INTO BARRIER $(0,0)$ & H-III, F05 \\
\hline$* 8035$ & 02 & FRONTAL & VEHICLE INTO BARRIER $(0,0)$ & H-III, F05 \\
\hline$* 8045$ & 02 & FRONTAL & VEHICLE INTO BARRIER $(0,0)$ & H-III, F05 \\
\hline *8055 & 02 & FRONTAL & VEHICLE INTO BARRIER $(0,0)$ & H-III, F05 \\
\hline$* 8064$ & 02 & FRONTAL & VEHICLE INTO BARRIER $(0,0)$ & H-III, F05 \\
\hline$* \uparrow 8068$ & 02 & FRONTAL & VEHICLE INTO BARRIER $(0,0)$ & H-III, F05 \\
\hline$* 8080$ & 02 & FRONTAL & VEHICLE INTO BARRIER $(0,0)$ & H-III, F05 \\
\hline$* 8081$ & 02 & FRONTAL & VEHICLE INTO BARRIER $(0,0)$ & H-III, F05 \\
\hline *8104 & 02 & FRONTAL & VEHICLE INTO BARRIER $(0,0)$ & H-III, F05 \\
\hline *8106 & 02 & FRONTAL & VEHICLE INTO BARRIER $(0,0)$ & H-III, F05 \\
\hline$* 8153$ & 02 & FRONTAL & VEHICLE INTO BARRIER $(0,0)$ & H-III, F05 \\
\hline$* 8156$ & 02 & FRONTAL & VEHICLE INTO BARRIER $(0,0)$ & H-III, F05 \\
\hline 5713 & 03 & FRONTAL & VEHICLE INTO BARRIER $(0,0)$ & H-III, M50 \\
\hline 5714 & 03 & FRONTAL & VEHICLE INTO BARRIER $(0,0)$ & H-III, M50 \\
\hline 5715 & 03 & FRONTAL & VEHICLE INTO BARRIER $(0,0)$ & H-III, M50 \\
\hline$* 6852$ & 04 & OBLIQUE & IMPACTOR INTO VEHICLE $(15,50)$ & H-III, F05 \\
\hline *6855 & 04 & OBLIQUE & IMPACTOR INTO VEHICLE $(15,18)$ & H-III, F05 \\
\hline$* 6865$ & 04 & OBLIQUE & VEHICLE INTO VEHICLE $(15,50)$ & H-III, F05 \\
\hline *6925 & 04 & OBLIQUE & IMPACTOR INTO VEHICLE $(15,10)$ & H-III, F05 \\
\hline
\end{tabular}




\begin{tabular}{|c|c|c|c|c|}
\hline$* 6937$ & 04 & OBLIQUE & IMPACTOR INTO VEHICLE $(15,50)$ & H-III, F05 \\
\hline *7366 & 04 & OBLIQUE & IMPACTOR INTO VEHICLE $(15,35)$ & H-III, F05 \\
\hline *7368 & 04 & OBLIQUE & IMPACTOR INTO VEHICLE $(7,20)$ & H-III, F05 \\
\hline *7428 & 04 & OBLIQUE & IMPACTOR INTO VEHICLE $(15,35)$ & H-III, F05 \\
\hline$* 7441$ & 04 & OBLIQUE & IMPACTOR INTO VEHICLE $(15,35)$ & H-III, F05 \\
\hline *7457 & 04 & OBLIQUE & IMPACTOR INTO VEHICLE $(15,35)$ & H-III, F05 \\
\hline$* 7427$ & 04 & OBLIQUE & IMPACTOR INTO VEHICLE $(7,20)$ & H-III, F05 \\
\hline *7432 & 04 & OBLIQUE & IMPACTOR INTO VEHICLE $(7,20)$ & H-III, F05 \\
\hline$* 7444$ & 04 & OBLIQUE & IMPACTOR INTO VEHICLE $(7,20)$ & H-III, F05 \\
\hline *7426 & 04 & OBLIQUE & IMPACTOR INTO VEHICLE $(7,20)$ & H-III, F05 \\
\hline *7456 & 04 & OBLIQUE & IMPACTOR INTO VEHICLE $(7,20)$ & H-III, F05 \\
\hline *7429 & 04 & OBLIQUE & IMPACTOR INTO VEHICLE $(15,35)$ & H-III, F05 \\
\hline *7434 & 04 & OBLIQUE & IMPACTOR INTO VEHICLE $(7,20)$ & H-III, F05 \\
\hline *7433 & 04 & OBLIQUE & IMPACTOR INTO VEHICLE $(15,35)$ & H-III, F05 \\
\hline *6872 & 04 & OBLIQUE & VEHICLE INTO IMPACTOR $(4,26)$ & H-III, F05 \\
\hline *7431 & 04 & OBLIQUE & IMPACTOR INTO VEHICLE $(15,35)$ & H-III, F05 \\
\hline *7467 & 04 & OBLIQUE & IMPACTOR INTO VEHICLE $(15,35)$ & H-III, F05 \\
\hline *7468 & 04 & OBLIQUE & IMPACTOR INTO VEHICLE $(7,20)$ & H-III, F05 \\
\hline *7476 & 04 & OBLIQUE & IMPACTOR INTO VEHICLE $(15,35)$ & H-III, F05 \\
\hline *8069 & 04 & SIDE & IMPACTOR INTO VEHICLE $(90,0)$ & SID-IIS, F05 \\
\hline *8078 & 04 & SIDE & IMPACTOR INTO VEHICLE $(90,0)$ & SID-IIS, F05 \\
\hline *8092 & 04 & SIDE & IMPACTOR INTO VEHICLE $(90,0)$ & SID-IIS, F05 \\
\hline *7955 & 01 & SIDE & VEHICLE INTO POLE $(90,0)$ & SID-IIS, F05 \\
\hline *7979 & 01 & SIDE & VEHICLE INTO POLE $(90,0)$ & SID-IIS, F05 \\
\hline *7988 & 01 & SIDE & VEHICLE INTO POLE $(90,0)$ & SID-IIS, F05 \\
\hline *7997 & 01 & SIDE & VEHICLE INTO POLE $(90,0)$ & SID-IIS, F05 \\
\hline$* 8052$ & 01 & SIDE & VEHICLE INTO POLE $(90,0)$ & SID-IIS, F05 \\
\hline 7851 & 01 & OBLIQUE & MDB; IMPACTOR INTO VEHICLE $(15,35)$ & THOR, M50 \\
\hline 7852 & 01 & OBLIQUE & MDB; IMPACTOR INTO VEHICLE $(15,35)$ & THOR, M50 \\
\hline 8085 & 01 & OBLIQUE & MDB; IMPACTOR INTO VEHICLE $(15,35)$ & THOR, M50 \\
\hline b8085 & 02 & OBLIQUE & MDB; IMPACTOR INTO VEHICLE $(15,35)$ & THOR, M50 \\
\hline 8099 & 01 & OBLIQUE & MDB; IMPACTOR INTO VEHICLE $(15,35)$ & THOR, M50 \\
\hline 8099 & 02 & OBLIQUE & MDB; IMPACTOR INTO VEHICLE $(15,35)$ & THOR, M50 \\
\hline 8475 & 01 & OBLIQUE & MDB; IMPACTOR INTO VEHICLE $(15,35)$ & THOR, M50 \\
\hline 8476 & 01 & OBLIQUE & MDB; IMPACTOR INTO VEHICLE $(15,35)$ & THOR, M50 \\
\hline 8477 & 01 & OBLIQUE & MDB; IMPACTOR INTO VEHICLE $(15,35)$ & THOR, M50 \\
\hline 8478 & 01 & OBLIQUE & MDB; IMPACTOR INTO VEHICLE $(15,35)$ & THOR, M50 \\
\hline 8478 & 02 & OBLIQUE & MDB; IMPACTOR INTO VEHICLE $(15,35)$ & THOR, M50 \\
\hline 8488 & 01 & OBLIQUE & MDB; IMPACTOR INTO VEHICLE $(15,35)$ & THOR, M50 \\
\hline 8488 & 02 & OBLIQUE & MDB; IMPACTOR INTO VEHICLE $(15,35)$ & THOR, M50 \\
\hline 8510 & 01 & OBLIQUE & MDB; IMPACTOR INTO VEHICLE $(7,20)$ & THOR, M50 \\
\hline 8512 & 01 & OBLIQUE & MDB; IMPACTOR INTO VEHICLE $(15,35)$ & THOR, M50 \\
\hline 8591 & 01 & OBLIQUE & MDB; IMPACTOR INTO VEHICLE $(15,35)$ & THOR, M50 \\
\hline 8787 & 01 & OBLIQUE & MDB; IMPACTOR INTO VEHICLE $(15,35)$ & THOR, M50 \\
\hline 8787 & 02 & OBLIQUE & MDB; IMPACTOR INTO VEHICLE $(15,35)$ & THOR, M50 \\
\hline 8788 & 01 & OBLIQUE & MDB; IMPACTOR INTO VEHICLE $(15,35)$ & THOR, M50 \\
\hline 8788 & 02 & OBLIQUE & MDB; IMPACTOR INTO VEHICLE $(15,35)$ & THOR, M50 \\
\hline 8789 & 01 & OBLIQUE & MDB; IMPACTOR INTO VEHICLE $(15,35)$ & THOR, M50 \\
\hline 8789 & 02 & OBLIQUE & MDB; IMPACTOR INTO VEHICLE $(15,35)$ & THOR, M50 \\
\hline 8791 & 01 & OBLIQUE & MDB; IMPACTOR INTO VEHICLE $(15,35)$ & THOR, M50 \\
\hline 8791 & 02 & OBLIQUE & MDB; IMPACTOR INTO VEHICLE $(15,35)$ & THOR, M50 \\
\hline 8998 & 01 & OBLIQUE & MDB; IMPACTOR INTO VEHICLE $(15,35)$ & THOR, M50 \\
\hline 8998 & 02 & OBLIQUE & MDB; IMPACTOR INTO VEHICLE $(15,35)$ & THOR, M50 \\
\hline 8999 & 01 & OBLIQUE & MDB; IMPACTOR INTO VEHICLE $(15,35)$ & THOR, M50 \\
\hline 8999 & 02 & OBLIQUE & MDB; IMPACTOR INTO VEHICLE $(15,35)$ & THOR, M50 \\
\hline 9042 & 01 & OBLIQUE & MDB; IMPACTOR INTO VEHICLE $(15,35)$ & THOR, M50 \\
\hline 9042 & 02 & OBLIQUE & MDB; IMPACTOR INTO VEHICLE $(15,35)$ & THOR, M50 \\
\hline 9043 & 01 & OBLIQUE & MDB; IMPACTOR INTO VEHICLE $(15,35)$ & THOR, M50 \\
\hline 9110 & 01 & OBLIQUE & MDB; IMPACTOR INTO VEHICLE $(15,35)$ & THOR, M50 \\
\hline 9110 & 02 & OBLIQUE & MDB; IMPACTOR INTO VEHICLE $(15,35)$ & THOR, M50 \\
\hline 9122 & 01 & OBLIQUE & MDB; IMPACTOR INTO VEHICLE $(15,35)$ & THOR, M50 \\
\hline 9122 & 02 & OBLIQUE & MDB; IMPACTOR INTO VEHICLE $(15,35)$ & THOR, M50 \\
\hline
\end{tabular}




\begin{tabular}{|c|c|c|c|c|}
\hline 9123 & 01 & OBLIQUE & MDB; IMPACTOR INTO VEHICLE $(15,35)$ & THOR, M50 \\
\hline 9123 & 02 & OBLIQUE & MDB; IMPACTOR INTO VEHICLE $(15,35)$ & THOR, M50 \\
\hline 9124 & 01 & OBLIQUE & MDB; IMPACTOR INTO VEHICLE $(15,35)$ & THOR, M50 \\
\hline 9124 & 02 & OBLIQUE & MDB; IMPACTOR INTO VEHICLE $(15,35)$ & THOR, M50 \\
\hline 9125 & 01 & OBLIQUE & MDB; IMPACTOR INTO VEHICLE $(15,35)$ & THOR, M50 \\
\hline 9125 & 02 & OBLIQUE & MDB; IMPACTOR INTO VEHICLE $(15,35)$ & THOR, M50 \\
\hline 9126 & 01 & OBLIQUE & MDB; IMPACTOR INTO VEHICLE $(15,35)$ & THOR, M50 \\
\hline 9126 & 02 & OBLIQUE & MDB; IMPACTOR INTO VEHICLE $(15,35)$ & THOR, M50 \\
\hline 9127 & 01 & OBLIQUE & MDB; IMPACTOR INTO VEHICLE $(15,35)$ & THOR, M50 \\
\hline 9127 & 02 & OBLIQUE & MDB; IMPACTOR INTO VEHICLE $(15,35)$ & THOR, M50 \\
\hline c9135 & 02 & OBLIQUE & MDB; IMPACTOR INTO VEHICLE $(15,35)$ & THOR, M50 \\
\hline 9137 & 01 & OBLIQUE & MDB; IMPACTOR INTO VEHICLE $(15,35)$ & THOR, M50 \\
\hline 9137 & 02 & OBLIQUE & MDB; IMPACTOR INTO VEHICLE $(15,35)$ & THOR, M50 \\
\hline 9138 & 01 & OBLIQUE & MDB; IMPACTOR INTO VEHICLE $(15,35)$ & THOR, M50 \\
\hline 9138 & 02 & OBLIQUE & MDB; IMPACTOR INTO VEHICLE $(15,35)$ & THOR, M50 \\
\hline 9139 & 01 & OBLIQUE & MDB; IMPACTOR INTO VEHICLE $(15,35)$ & THOR, M50 \\
\hline 9139 & 02 & OBLIQUE & MDB; IMPACTOR INTO VEHICLE $(15,35)$ & THOR, M50 \\
\hline 9140 & 01 & OBLIQUE & MDB; IMPACTOR INTO VEHICLE $(15,35)$ & THOR, M50 \\
\hline 9140 & 02 & OBLIQUE & MDB; IMPACTOR INTO VEHICLE $(15,35)$ & THOR, M50 \\
\hline 9142 & 01 & OBLIQUE & MDB; IMPACTOR INTO VEHICLE $(15,35)$ & THOR, M50 \\
\hline 9143 & 01 & OBLIQUE & MDB; IMPACTOR INTO VEHICLE $(15,35)$ & THOR, M50 \\
\hline 9144 & 01 & OBLIQUE & MDB; IMPACTOR INTO VEHICLE $(15,35)$ & THOR, M50 \\
\hline 9144 & 02 & OBLIQUE & MDB; IMPACTOR INTO VEHICLE $(15,35)$ & THOR, M50 \\
\hline 9145 & 01 & OBLIQUE & MDB; IMPACTOR INTO VEHICLE $(15,35)$ & THOR, M50 \\
\hline 9146 & 01 & OBLIQUE & MDB; IMPACTOR INTO VEHICLE $(15,35)$ & THOR, M50 \\
\hline 9146 & 02 & OBLIQUE & MDB; IMPACTOR INTO VEHICLE $(15,35)$ & THOR, M50 \\
\hline 9147 & 02 & OBLIQUE & MDB; IMPACTOR INTO VEHICLE $(15,35)$ & THOR, M50 \\
\hline 9148 & 01 & OBLIQUE & MDB; IMPACTOR INTO VEHICLE $(15,35)$ & THOR, M50 \\
\hline 9148 & 02 & OBLIQUE & MDB; IMPACTOR INTO VEHICLE $(15,35)$ & THOR, M50 \\
\hline 9149 & 01 & OBLIQUE & MDB; IMPACTOR INTO VEHICLE $(15,35)$ & THOR, M50 \\
\hline 9149 & 02 & OBLIQUE & MDB; IMPACTOR INTO VEHICLE $(15,35)$ & THOR, M50 \\
\hline 9150 & 01 & OBLIQUE & MDB; IMPACTOR INTO VEHICLE $(15,35)$ & THOR, M50 \\
\hline 9150 & 02 & OBLIQUE & MDB; IMPACTOR INTO VEHICLE $(15,35)$ & THOR, M50 \\
\hline 9151 & 01 & OBLIQUE & MDB; IMPACTOR INTO VEHICLE $(15,35)$ & THOR, M50 \\
\hline 9151 & 02 & OBLIQUE & MDB; IMPACTOR INTO VEHICLE $(15,35)$ & THOR, M50 \\
\hline 9152 & 01 & OBLIQUE & MDB; IMPACTOR INTO VEHICLE $(15,35)$ & THOR, M50 \\
\hline 9152 & 02 & OBLIQUE & MDB; IMPACTOR INTO VEHICLE $(15,35)$ & THOR, M50 \\
\hline 9155 & 01 & OBLIQUE & MDB; IMPACTOR INTO VEHICLE $(15,35)$ & THOR, M50 \\
\hline 9155 & 02 & OBLIQUE & MDB; IMPACTOR INTO VEHICLE $(15,35)$ & THOR, M50 \\
\hline 9219 & 01 & OBLIQUE & MDB; IMPACTOR INTO VEHICLE $(7,20)$ & THOR, M50 \\
\hline 9220 & 01 & OBLIQUE & MDB; IMPACTOR INTO VEHICLE $(7,20)$ & THOR, M50 \\
\hline *9220 & 04 & OBLIQUE & MDB; IMPACTOR INTO VEHICLE $(7,20)$ & H-III, F05 \\
\hline 9221 & 01 & OBLIQUE & MDB; IMPACTOR INTO VEHICLE $(7,20)$ & THOR, M50 \\
\hline *9221 & 04 & OBLIQUE & MDB; IMPACTOR INTO VEHICLE $(7,20)$ & H-III, F05 \\
\hline 9222 & 01 & OBLIQUE & MDB; IMPACTOR INTO VEHICLE $(7,20)$ & THOR, M50 \\
\hline 9223 & 01 & OBLIQUE & MDB; IMPACTOR INTO VEHICLE $(15,35)$ & THOR, M50 \\
\hline a9223 & 02 & OBLIQUE & MDB; IMPACTOR INTO VEHICLE $(15,35)$ & THOR, M50 \\
\hline 9333 & 01 & FRONTAL & VEHICLE INTO BARRIER $(0,0)$ & THOR, M50 \\
\hline *9333 & 02 & FRONTAL & VEHICLE INTO BARRIER $(0,0)$ & H-III, F05 \\
\hline 9334 & 01 & FRONTAL & VEHICLE INTO BARRIER $(0,0)$ & THOR, M50 \\
\hline *9334 & 02 & FRONTAL & VEHICLE INTO BARRIER $(0,0)$ & H-III, F05 \\
\hline 9335 & 01 & FRONTAL & VEHICLE INTO BARRIER $(0,0)$ & THOR, M50 \\
\hline *9335 & 02 & FRONTAL & VEHICLE INTO BARRIER $(0,0)$ & H-III, F05 \\
\hline 9336 & 01 & FRONTAL & VEHICLE INTO BARRIER $(0,0)$ & THOR, M50 \\
\hline c*9336 & 02 & FRONTAL & VEHICLE INTO BARRIER $(0,0)$ & H-III, F05 \\
\hline 9337 & 01 & FRONTAL & VEHICLE INTO BARRIER $(0,0)$ & THOR, M50 \\
\hline *9337 & 02 & FRONTAL & VEHICLE INTO BARRIER $(0,0)$ & H-III, F05 \\
\hline 9354 & 01 & OBLIQUE & MDB; IMPACTOR INTO VEHICLE $(15,35)$ & THOR, M50 \\
\hline 9354 & 02 & OBLIQUE & MDB; IMPACTOR INTO VEHICLE $(15,35)$ & THOR, M50 \\
\hline 9476 & 01 & OBLIQUE & MDB; IMPACTOR INTO VEHICLE $(15,35)$ & THOR, M50 \\
\hline 9476 & 02 & OBLIQUE & MDB; IMPACTOR INTO VEHICLE $(15,35)$ & THOR, M50 \\
\hline 9477 & 01 & OBLIQUE & MDB; IMPACTOR INTO VEHICLE $(15,35)$ & THOR, M50 \\
\hline
\end{tabular}




\begin{tabular}{|c|c|c|c|c|}
\hline 9477 & 02 & OBLIQUE & MDB; IMPACTOR INTO VEHICLE $(15,35)$ & THOR, M50 \\
\hline 9478 & 01 & OBLIQUE & MDB; IMPACTOR INTO VEHICLE $(15,35)$ & THOR, M50 \\
\hline 9478 & 02 & OBLIQUE & MDB; IMPACTOR INTO VEHICLE $(15,35)$ & THOR, M50 \\
\hline 9479 & 01 & OBLIQUE & MDB; IMPACTOR INTO VEHICLE $(15,35)$ & THOR, M50 \\
\hline 9479 & 02 & OBLIQUE & MDB; IMPACTOR INTO VEHICLE $(15,35)$ & THOR, M50 \\
\hline 9480 & 01 & OBLIQUE & MDB; IMPACTOR INTO VEHICLE $(15,35)$ & THOR, M50 \\
\hline 9480 & 02 & OBLIQUE & MDB; IMPACTOR INTO VEHICLE $(15,35)$ & THOR, M50 \\
\hline 9481 & 01 & OBLIQUE & MDB; IMPACTOR INTO VEHICLE $(15,35)$ & THOR, M50 \\
\hline 9481 & 02 & OBLIQUE & MDB; IMPACTOR INTO VEHICLE $(15,35)$ & THOR, M50 \\
\hline 9482 & 01 & OBLIQUE & MDB; IMPACTOR INTO VEHICLE $(15,35)$ & THOR, M50 \\
\hline 9482 & 02 & OBLIQUE & MDB; IMPACTOR INTO VEHICLE $(15,35)$ & THOR, M50 \\
\hline 9483 & 01 & OBLIQUE & MDB; IMPACTOR INTO VEHICLE $(15,35)$ & THOR, M50 \\
\hline 9483 & 02 & OBLIQUE & MDB; IMPACTOR INTO VEHICLE $(15,35)$ & THOR, M50 \\
\hline 9499 & 01 & OBLIQUE & MDB; IMPACTOR INTO VEHICLE $(15,35)$ & THOR, M50 \\
\hline 9499 & 02 & OBLIQUE & MDB; IMPACTOR INTO VEHICLE $(15,35)$ & THOR, M50 \\
\hline 9500 & 01 & OBLIQUE & MDB; IMPACTOR INTO VEHICLE $(15,35)$ & THOR, M50 \\
\hline 9500 & 02 & OBLIQUE & MDB; IMPACTOR INTO VEHICLE $(15,35)$ & THOR, M50 \\
\hline 9501 & 01 & OBLIQUE & MDB; IMPACTOR INTO VEHICLE $(15,35)$ & THOR, M50 \\
\hline 9501 & 02 & OBLIQUE & MDB; IMPACTOR INTO VEHICLE $(15,35)$ & THOR, M50 \\
\hline 9566 & 01 & FRONTAL & VEHICLE INTO BARRIER $(0,0)$ & THOR, M50 \\
\hline c*9566 & 02 & FRONTAL & VEHICLE INTO BARRIER $(0,0)$ & H-III, F05 \\
\hline d9567 & 01 & FRONTAL & VEHICLE INTO BARRIER $(0,0)$ & THOR, M50 \\
\hline *9567 & 02 & FRONTAL & VEHICLE INTO BARRIER $(0,0)$ & H-III, F05 \\
\hline 9568 & 01 & FRONTAL & VEHICLE INTO BARRIER $(0,0)$ & THOR, M50 \\
\hline *9568 & 02 & FRONTAL & VEHICLE INTO BARRIER $(0,0)$ & H-III, F05 \\
\hline 9569 & 01 & FRONTAL & VEHICLE INTO BARRIER $(0,0)$ & THOR, M50 \\
\hline *9569 & 02 & FRONTAL & VEHICLE INTO BARRIER $(0,0)$ & H-III, F05 \\
\hline 9570 & 01 & FRONTAL & VEHICLE INTO BARRIER $(0,0)$ & THOR, M50 \\
\hline$* 9570$ & 02 & FRONTAL & VEHICLE INTO BARRIER $(0,0)$ & H-III, F05 \\
\hline 9571 & 01 & FRONTAL & VEHICLE INTO BARRIER $(0,0)$ & THOR, M50 \\
\hline *9571 & 02 & FRONTAL & VEHICLE INTO BARRIER $(0,0)$ & H-III, F05 \\
\hline 9572 & 01 & OBLIQUE & MDB; IMPACTOR INTO VEHICLE $(15,35)$ & THOR, M50 \\
\hline 9572 & 02 & OBLIQUE & MDB; IMPACTOR INTO VEHICLE $(15,35)$ & THOR, M50 \\
\hline c9573 & 01 & OBLIQUE & MDB; IMPACTOR INTO VEHICLE $(15,35)$ & THOR, M50 \\
\hline 9573 & 02 & OBLIQUE & MDB; IMPACTOR INTO VEHICLE $(15,35)$ & THOR, M50 \\
\hline 9574 & 01 & OBLIQUE & MDB; IMPACTOR INTO VEHICLE $(15,35)$ & THOR, M50 \\
\hline 9574 & 02 & OBLIQUE & MDB; IMPACTOR INTO VEHICLE $(15,35)$ & THOR, M50 \\
\hline 9585 & 01 & OBLIQUE & MDB; IMPACTOR INTO VEHICLE $(15,35)$ & THOR, M50 \\
\hline 9585 & 02 & OBLIQUE & MDB; IMPACTOR INTO VEHICLE $(15,35)$ & THOR, M50 \\
\hline 9586 & 01 & OBLIQUE & MDB; IMPACTOR INTO VEHICLE $(15,35)$ & THOR, M50 \\
\hline 9586 & 02 & OBLIQUE & MDB; IMPACTOR INTO VEHICLE $(15,35)$ & THOR, M50 \\
\hline 9587 & 01 & OBLIQUE & MDB; IMPACTOR INTO VEHICLE $(15,35)$ & THOR, M50 \\
\hline 9587 & 02 & OBLIQUE & MDB; IMPACTOR INTO VEHICLE $(15,35)$ & THOR, M50 \\
\hline 9699 & 01 & OBLIQUE & MDB; IMPACTOR INTO VEHICLE $(15,35)$ & THOR, M50 \\
\hline 9699 & 02 & OBLIQUE & MDB; IMPACTOR INTO VEHICLE $(15,35)$ & THOR, M50 \\
\hline 9726 & 01 & OBLIQUE & MDB; IMPACTOR INTO VEHICLE $(15,35)$ & THOR, M50 \\
\hline 9726 & 02 & OBLIQUE & MDB; IMPACTOR INTO VEHICLE $(15,35)$ & THOR, M50 \\
\hline 9727 & 01 & OBLIQUE & MDB; IMPACTOR INTO VEHICLE $(15,35)$ & THOR, M50 \\
\hline 9727 & 02 & OBLIQUE & MDB; IMPACTOR INTO VEHICLE $(15,35)$ & THOR, M50 \\
\hline 9802 & 01 & OBLIQUE & MDB; IMPACTOR INTO VEHICLE $(15,35)$ & THOR, M50 \\
\hline 9802 & 02 & OBLIQUE & MDB; IMPACTOR INTO VEHICLE $(15,35)$ & THOR, M50 \\
\hline 9804 & 01 & OBLIQUE & MDB; IMPACTOR INTO VEHICLE $(15,35)$ & THOR, M50 \\
\hline 9804 & 02 & OBLIQUE & MDB; IMPACTOR INTO VEHICLE $(15,35)$ & THOR, M50 \\
\hline 9806 & 01 & OBLIQUE & MDB; IMPACTOR INTO VEHICLE $(15,35)$ & THOR, M50 \\
\hline 9806 & 02 & OBLIQUE & MDB; IMPACTOR INTO VEHICLE $(15,35)$ & THOR, M50 \\
\hline
\end{tabular}

Occupant Seat Location (Occ. Loc.): 01 = left front, 02 = right front, $03=$ right rear, $04=$ left rear (\#\#, \#\#) indicates impact parameters (degree offset, percent overlap)

*Kinematics scaled to 50TH male using the equal-stress-equal-velocity scaling technique.

Data time histories were truncated to $175 \mathrm{~ms}(\dagger), 190 \mathrm{~ms}(\mathrm{a}), 200 \mathrm{~ms}(\mathrm{~b}), 250 \mathrm{~ms}(\mathrm{c}), 275 \mathrm{~ms}(\mathrm{~d})$ to avoid impacts that may have been developing. 


\section{I.3 NHTSA Pendulum Impactor Tests}

\begin{tabular}{|c|c|c|c|}
\hline Source ID & $\begin{array}{c}\text { Impact } \\
\text { Condition }\end{array}$ & Test Details & Surrogate \\
\hline ES2-1 & SIDE & UNPADDED IMPACTOR AT $90^{\circ}$ INTO HEAD CG & ES-2RE, M50 \\
\hline ES2-2 & SIDE & UNPADDED IMPACTOR AT $90^{\circ}$ INTO HEAD CG & ES-2RE, M50 \\
\hline ES2-3 & OBLIQUE & UNPADDED IMPACTOR AT $60^{\circ}$ INTO HEAD CG & ES-2RE, M50 \\
\hline ES2-4 & OBLIQUE & UNPADDED IMPACTOR AT $60^{\circ}$ INTO HEAD CG & ES-2RE, M50 \\
\hline ES2-5 & OBLIQUE & UNPADDED IMPACTOR AT $60^{\circ}$ INTO HEAD CG & ES-2RE, M50 \\
\hline ES2-6 & SIDE & PADDED IMPACTOR AT 90 INTO HEAD CG & ES-2RE, M50 \\
\hline ES2-7 & OBLIQUE & PADDED IMPACTOR AT $60^{\circ}$ INTO HEAD CG & ES-2RE, M50 \\
\hline ES2-8 & OBLIQUE & UNPADDED IMPACTOR AT $60^{\circ}$ INTO HEAD CG & ES-2RE, M50 \\
\hline ES2-9 & SIDE & PADDED IMPACTOR AT 90 INTO HEAD CG & ES-2RE, M50 \\
\hline ES2-10 & OBLIQUE & PADDED IMPACTOR AT $60^{\circ}$ INTO HEAD CG & ES-2RE, M50 \\
\hline ES2-11 & SIDE & UNPADDED IMPACTOR AT $90^{\circ}$ INTO HEAD CG & ES-2RE, M50 \\
\hline ES2-12 & SIDE & PADDED IMPACTOR AT $90^{\circ}$ INTO HEAD CG & ES-2RE, M50 \\
\hline ES2-13 & OBLIQUE & UNPADDED IMPACTOR AT $60^{\circ}$ INTO HEAD CG & ES-2RE, M50 \\
\hline ES2-14 & OBLIQUE & PADDED IMPACTOR AT $60^{\circ}$ INTO HEAD CG & ES-2RE, M50 \\
\hline ES2-15 & SIDE & UNPADDED IMPACTOR AT $90^{\circ}$ INTO HEAD CG & ES-2RE, M50 \\
\hline ES2-16 & SIDE & PADDED IMPACTOR AT $90^{\circ}$ INTO HEAD CG & ES-2RE, M50 \\
\hline ES2-17 & OBLIQUE & PADDED IMPACTOR AT $60^{\circ}$ INTO HEAD CG & ES-2RE, M50 \\
\hline ES2-18 & SIDE & UNPADDED IMPACTOR AT $90^{\circ}$ INTO HEAD CG & ES-2RE, M50 \\
\hline ES2-19 & SIDE & PADDED IMPACTOR AT $90^{\circ}$ INTO HEAD CG & ES-2RE, M50 \\
\hline ES2-20 & OBLIQUE & PADDED IMPACTOR AT $60^{\circ}$ INTO HEAD CG & ES-2RE, M50 \\
\hline $\mathrm{H} 3-5-1$ & FRONTAL & PADDED IMPACTOR AT $0^{\circ}$ INTO HEAD CG & H-III, F05 \\
\hline $\mathrm{H} 3-5-2$ & FRONTAL & PADDED IMPACTOR AT $0^{\circ}$ INTO HEAD CG & H-III, F05 \\
\hline $\mathrm{H} 3-5-3$ & FRONTAL & PADDED IMPACTOR AT $0^{\circ}$ INTO HEAD CG & H-III, F05 \\
\hline $\mathrm{H} 3-5-4$ & FRONTAL & PADDED IMPACTOR AT $0^{\circ}$ INTO HEAD CG & H-III, F05 \\
\hline $\mathrm{H} 3-5-5$ & FRONTAL & PADDED IMPACTOR AT $0^{\circ}$ INTO HEAD CG & H-III, F05 \\
\hline $\mathrm{H} 3-5-7$ & OBLIQUE & PADDED IMPACTOR AT $30^{\circ}$ INTO HEAD CG & H-III, F05 \\
\hline H3-5-8 & OBLIQUE & PADDED IMPACTOR AT $30^{\circ}$ INTO HEAD CG & H-III, F05 \\
\hline H3-5-6 & OBLIQUE & PADDED IMPACTOR AT $30^{\circ}$ INTO HEAD CG & H-III, F05 \\
\hline H3-5-9 & OBLIQUE & PADDED IMPACTOR AT $30^{\circ}$ INTO HEAD CG & H-III, F05 \\
\hline $\mathrm{H} 3-5-10$ & OBLIQUE & PADDED IMPACTOR AT 30 INTO HEAD CG & H-III, F05 \\
\hline H3-5-11 & FRONTAL & UNPADDED IMPACTOR AT $0^{\circ}$ INTO HEAD CG & H-III, F05 \\
\hline $\mathrm{H} 3-5-12$ & FRONTAL & UNPADDED IMPACTOR AT $0^{\circ}$ INTO HEAD CG & H-III, F05 \\
\hline $\mathrm{H} 3-5-13$ & FRONTAL & UNPADDED IMPACTOR AT $0^{\circ}$ INTO HEAD CG & H-III, F05 \\
\hline $\mathrm{H} 3-5-14$ & FRONTAL & UNPADDED IMPACTOR AT $0^{\circ}$ INTO HEAD CG & H-III, F05 \\
\hline H3-5-15 & FRONTAL & UNPADDED IMPACTOR AT $0^{\circ}$ INTO HEAD CG & H-III, F05 \\
\hline H3-5-16 & FRONTAL & UNPADDED IMPACTOR AT $0^{\circ}$ INTO HEAD CG & H-III, F05 \\
\hline H3-5-17 & OBLIQUE & UNPADDED IMPACTOR AT $30^{\circ}$ INTO HEAD CG & H-III, F05 \\
\hline H3-5-18 & OBLIQUE & UNPADDED IMPACTOR AT $30^{\circ}$ INTO HEAD CG & H-III, F05 \\
\hline H3-5-19 & OBLIQUE & UNPADDED IMPACTOR AT $30^{\circ}$ INTO HEAD CG & H-III, F05 \\
\hline $\mathrm{H} 3-5-20$ & OBLIQUE & UNPADDED IMPACTOR AT $30^{\circ}$ INTO HEAD CG & H-III, F05 \\
\hline $\mathrm{H} 3-5-21$ & OBLIQUE & UNPADDED IMPACTOR AT $30^{\circ}$ INTO HEAD CG & H-III, F05 \\
\hline $\mathrm{H} 3-5-22$ & FRONTAL & PADDED IMPACTOR AT $0^{\circ}$ INTO HEAD CG & H-III, F05 \\
\hline $\mathrm{H} 3-5-23$ & OBLIQUE & PADDED IMPACTOR AT 30 INTO HEAD CG & H-III, F05 \\
\hline SID2S-1 & SIDE & UNPADDED IMPACTOR AT $90^{\circ}$ INTO HEAD CG & SID-IIS, F05 \\
\hline SID2S-2 & SIDE & PADDED IMPACTOR AT $90^{\circ}$ INTO HEAD CG & SID-IIS, F05 \\
\hline SID2S-3 & OBLIQUE & UNPADDED IMPACTOR AT $60^{\circ}$ INTO HEAD CG & SID-IIS, F05 \\
\hline SID2S-4 & OBLIQUE & PADDED IMPACTOR AT $60^{\circ}$ INTO HEAD CG & SID-IIS, F05 \\
\hline SID2S-5 & SIDE & UNPADDED IMPACTOR AT $90^{\circ}$ INTO HEAD CG & SID-IIS, F05 \\
\hline SID2S-6 & SIDE & PADDED IMPACTOR AT 90 INTO HEAD CG & SID-IIS, F05 \\
\hline SID2S-7 & OBLIQUE & UNPADDED IMPACTOR AT $60^{\circ}$ INTO HEAD CG & SID-IIS, F05 \\
\hline SID2S-8 & SIDE & UNPADDED IMPACTOR AT $90^{\circ}$ INTO HEAD CG & SID-IIS, F05 \\
\hline SID2S-9 & OBLIQUE & UNPADDED IMPACTOR AT $60^{\circ}$ INTO HEAD CG & SID-IIS, F05 \\
\hline SID2S-10 & OBLIQUE & PADDED IMPACTOR AT $60^{\circ}$ INTO HEAD CG & SID-IIS, F05 \\
\hline SID2S-11 & SIDE & PADDED IMPACTOR AT $90^{\circ}$ INTO HEAD CG & SID-IIS, F05 \\
\hline SID2S-12 & OBLIQUE & PADDED IMPACTOR AT $60^{\circ}$ INTO HEAD CG & SID-IIS, F05 \\
\hline SID2S-13 & SIDE & UNPADDED IMPACTOR AT $90^{\circ}$ INTO HEAD CG & SID-IIS, F05 \\
\hline SID2S-14 & OBLIQUE & UNPADDED IMPACTOR AT $60^{\circ}$ INTO HEAD CG & SID-IIS, F05 \\
\hline
\end{tabular}




\begin{tabular}{|c|c|c|c|}
\hline SID2S-15 & SIDE & PADDED IMPACTOR AT $90^{\circ}$ INTO HEAD CG & SID-IIS, F05 \\
\hline SID2S-16 & SIDE & PADDED IMPACTOR AT $90^{\circ}$ INTO HEAD CG & SID-IIS, F05 \\
\hline SID2S-17 & OBLIQUE & PADDED IMPACTOR AT $60^{\circ}$ INTO HEAD CG & SID-IIS, F05 \\
\hline SID2S-18 & SIDE & UNPADDED IMPACTOR AT $90^{\circ}$ INTO HEAD CG & SID-IIS, F05 \\
\hline SID2S-19 & OBLIQUE & UNPADDED IMPACTOR AT $60^{\circ}$ INTO HEAD CG & SID-IIS, F05 \\
\hline SID2S-20 & OBLIQUE & PADDED IMPACTOR AT $60^{\circ}$ INTO HEAD CG & SID-IIS, F05 \\
\hline SID2S-21 & SIDE & PADDED IMPACTOR AT $90^{\circ}$ INTO HEAD CG & SID-IIS, F05 \\
\hline THOR-1 & FRONTAL & PADDED IMPACTOR AT $0^{\circ}$ INTO HEAD CG & THOR, M50 \\
\hline THOR-2 & FRONTAL & PADDED IMPACTOR AT $0^{\circ}$ INTO FOREHEAD & THOR, M50 \\
\hline THOR-3 & FRONTAL & PADDED IMPACTOR AT $0^{\circ}$ INTO FOREHEAD & THOR, M50 \\
\hline THOR-4 & FRONTAL & PADDED IMPACTOR AT $0^{\circ}$ INTO FOREHEAD & THOR, M50 \\
\hline THOR-5 & FRONTAL & PADDED IMPACTOR AT $0^{\circ}$ INTO FOREHEAD & THOR, M50 \\
\hline THOR-6 & FRONTAL & PADDED IMPACTOR AT $0^{\circ}$ INTO FOREHEAD & THOR, M50 \\
\hline THOR-7 & OBLIQUE & PADDED IMPACTOR AT $30^{\circ}$ INTO FOREHEAD & THOR, M50 \\
\hline THOR-8 & OBLIQUE & PADDED IMPACTOR AT $30^{\circ}$ INTO FOREHEAD & THOR, M50 \\
\hline THOR-9 & OBLIQUE & PADDED IMPACTOR AT $30^{\circ}$ INTO FOREHEAD & THOR, M50 \\
\hline THOR-10 & OBLIQUE & PADDED IMPACTOR AT $30^{\circ}$ INTO FOREHEAD & THOR, M50 \\
\hline THOR-11 & OBLIQUE & PADDED IMPACTOR AT $30^{\circ}$ INTO FOREHEAD & THOR, M50 \\
\hline THOR-12 & OBLIQUE & PADDED IMPACTOR AT $30^{\circ}$ INTO FOREHEAD & THOR, M50 \\
\hline THOR-13 & FRONTAL & PADDED IMPACTOR AT 0 INTO FOREHEAD & THOR, M50 \\
\hline THOR-14 & FRONTAL & UNPADDED IMPACTOR AT $0^{\circ}$ INTO FOREHEAD & THOR, M50 \\
\hline THOR-15 & OBLIQUE & UNPADDED IMPACTOR AT $30^{\circ}$ INTO FOREHEAD & THOR, M50 \\
\hline THOR-16 & FRONTAL & UNPADDED IMPACTOR AT $0^{\circ}$ INTO FOREHEAD & THOR, M50 \\
\hline THOR-17 & OBLIQUE & UNPADDED IMPACTOR AT $30^{\circ}$ INTO FOREHEAD & THOR, M50 \\
\hline THOR-18 & FRONTAL & UNPADDED IMPACTOR AT $0^{\circ}$ INTO FOREHEAD & THOR, M50 \\
\hline THOR-19 & OBLIQUE & UNPADDED IMPACTOR AT $30^{\circ}$ INTO FOREHEAD & THOR, M50 \\
\hline THOR-20 & FRONTAL & UNPADDED IMPACTOR AT $0^{\circ}$ INTO FOREHEAD & THOR, M50 \\
\hline THOR-21 & OBLIQUE & UNPADDED IMPACTOR AT $30^{\circ}$ INTO FOREHEAD & THOR, M50 \\
\hline THOR-22 & FRONTAL & UNPADDED IMPACTOR AT $0^{\circ}$ INTO FOREHEAD & THOR, M50 \\
\hline THOR-23 & OBLIQUE & UNPADDED IMPACTOR AT $30^{\circ}$ INTO FOREHEAD & THOR, M50 \\
\hline THOR-24 & FRONTAL & UNPADDED IMPACTOR AT $0^{\circ}$ INTO FOREHEAD & THOR, M50 \\
\hline THOR-25 & OBLIQUE & UNPADDED IMPACTOR AT $30^{\circ}$ INTO FOREHEAD & THOR, M50 \\
\hline WS5-1 & SIDE & UNPADDED IMPACTOR AT 90 INTO HEAD CG & WSID, F05 \\
\hline WS5-2 & SIDE & PADDED IMPACTOR AT 90 INTO HEAD CG & WSID, F05 \\
\hline WS5-3 & SIDE & UNPADDED IMPACTOR AT $90^{\circ}$ INTO HEAD CG & WSID, F05 \\
\hline WS5-4 & OBLIQUE & PADDED IMPACTOR AT $60^{\circ}$ INTO HEAD CG & WSID, F05 \\
\hline WS5-5 & OBLIQUE & UNPADDED IMPACTOR AT $60^{\circ}$ INTO HEAD CG & WSID, F05 \\
\hline WS5-6 & OBLIQUE & PADDED IMPACTOR AT $60^{\circ}$ INTO HEAD CG & WSID, F05 \\
\hline WS5-7 & OBLIQUE & UNPADDED IMPACTOR AT $60^{\circ}$ INTO HEAD CG & WSID, F05 \\
\hline WS5-8 & OBLIQUE & UNPADDED IMPACTOR AT $60^{\circ}$ INTO HEAD CG & WSID, F05 \\
\hline WS5-9 & SIDE & PADDED IMPACTOR AT $90^{\circ}$ INTO HEAD CG & WSID, F05 \\
\hline WS5-10 & SIDE & UNPADDED IMPACTOR AT $90^{\circ}$ INTO HEAD CG & WSID, F05 \\
\hline WS5-11 & SIDE & PADDED IMPACTOR AT $90^{\circ}$ INTO HEAD CG & WSID, F05 \\
\hline WS5-13 & OBLIQUE & PADDED IMPACTOR AT $60^{\circ}$ INTO HEAD CG & WSID, F05 \\
\hline WS5-14 & SIDE & UNPADDED IMPACTOR AT $90^{\circ}$ INTO HEAD CG & WSID, F05 \\
\hline WS5-15 & OBLIQUE & UNPADDED IMPACTOR AT $60^{\circ}$ INTO HEAD CG & WSID, F05 \\
\hline WS5-16 & SIDE & PADDED IMPACTOR AT 90 INTO HEAD CG & WSID, F05 \\
\hline WS5-17 & OBLIQUE & PADDED IMPACTOR AT $60^{\circ}$ INTO HEAD CG & WSID, F05 \\
\hline WS5-18 & SIDE & UNPADDED IMPACTOR AT $90^{\circ}$ INTO HEAD CG & WSID, F05 \\
\hline WS5-19 & OBLIQUE & UNPADDED IMPACTOR AT $60^{\circ}$ INTO HEAD CG & WSID, F05 \\
\hline WS5-20 & SIDE & PADDED IMPACTOR AT $90^{\circ}$ INTO HEAD CG & WSID, F05 \\
\hline WS5-21 & OBLIQUE & PADDED IMPACTOR AT $60^{\circ}$ INTO HEAD CG & WSID, F05 \\
\hline W50-1 & SIDE & PADDED IMPACTOR AT $90^{\circ}$ INTO HEAD CG & WSID, M50 \\
\hline W50-2 & SIDE & PADDED IMPACTOR AT $90^{\circ}$ INTO HEAD CG & WSID, M50 \\
\hline W50-3 & SIDE & PADDED IMPACTOR AT $90^{\circ}$ INTO HEAD CG & WSID, M50 \\
\hline W50-4 & SIDE & PADDED IMPACTOR AT $90^{\circ}$ INTO HEAD CG & WSID, M50 \\
\hline W50-5 & SIDE & PADDED IMPACTOR AT $90^{\circ}$ INTO HEAD CG & WSID, M50 \\
\hline W50-6 & OBLIQUE & PADDED IMPACTOR AT $60^{\circ}$ INTO HEAD CG & WSID, M50 \\
\hline W50-7 & OBLIQUE & PADDED IMPACTOR AT $60^{\circ}$ INTO HEAD CG & WSID, M50 \\
\hline W50-8 & OBLIQUE & PADDED IMPACTOR AT $60^{\circ}$ INTO HEAD CG & WSID, M50 \\
\hline W50-9 & OBLIQUE & PADDED IMPACTOR AT $60^{\circ}$ INTO HEAD CG & WSID, M50 \\
\hline W50-10 & OBLIQUE & PADDED IMPACTOR AT $60^{\circ}$ INTO HEAD CG & WSID, M50 \\
\hline
\end{tabular}




\begin{tabular}{|c|c|c|c|}
\hline W50-11 & SIDE & UNPADDED IMPACTOR AT 90 INTO HEAD CG & WSID, M50 \\
\hline W50-12 & SIDE & UNPADDED IMPACTOR AT $90^{\circ}$ INTO HEAD CG & WSID, M50 \\
\hline W50-13 & SIDE & UNPADDED IMPACTOR AT $90^{\circ}$ INTO HEAD CG & WSID, M50 \\
\hline W50-14 & SIDE & UNPADDED IMPACTOR AT $90^{\circ}$ INTO HEAD CG & WSID, M50 \\
\hline W50-15 & SIDE & UNPADDED IMPACTOR AT $90^{\circ}$ INTO HEAD CG & WSID, M50 \\
\hline W50-16 & OBLIQUE & UNPADDED IMPACTOR AT $60^{\circ}$ INTO HEAD CG & WSID, M50 \\
\hline W50-17 & OBLIQUE & UNPADDED IMPACTOR AT $60^{\circ}$ INTO HEAD CG & WSID, M50 \\
\hline W50-18 & OBLIQUE & UNPADDED IMPACTOR AT $60^{\circ}$ INTO HEAD CG & WSID, M50 \\
\hline W50-19 & OBLIQUE & UNPADDED IMPACTOR AT $60^{\circ}$ INTO HEAD CG & WSID, M50 \\
\hline W50-20 & OBLIQUE & UNPADDED IMPACTOR AT $60^{\circ}$ INTO HEAD CG & WSID, M50 \\
\hline W50-21 & OBLIQUE & UNPADDED IMPACTOR AT $60^{\circ}$ INTO HEAD CG & WSID, M50 \\
\hline H3-50-37 & FRONTAL & UNPADDED IMPACTOR AT $0^{\circ}$ INTO HEAD CG & H-III, M50 \\
\hline $\mathrm{H} 3-50-53$ & FRONTAL & UNPADDED IMPACTOR AT $0^{\circ}$ INTO HEAD CG & H-III, M50 \\
\hline H3-50-9 & FRONTAL & UNPADDED IMPACTOR AT $0^{\circ}$ INTO HEAD CG & H-III, M50 \\
\hline H3-50-21 & FRONTAL & UNPADDED IMPACTOR AT $0^{\circ}$ INTO HEAD CG & H-III, M50 \\
\hline $\mathrm{H} 3-50-49$ & FRONTAL & PADDED IMPACTOR AT $0^{\circ}$ INTO HEAD CG & H-III, M50 \\
\hline H3-50-5 & FRONTAL & PADDED IMPACTOR AT $0^{\circ}$ INTO HEAD CG & H-III, M50 \\
\hline H3-50-13 & FRONTAL & PADDED IMPACTOR AT $0^{\circ}$ INTO HEAD CG & H-III, M50 \\
\hline H3-50-41 & FRONTAL & PADDED IMPACTOR AT $0^{\circ}$ INTO HEAD CG & H-III, M50 \\
\hline H3-50-33 & FRONTAL & PADDED IMPACTOR AT $0^{\circ}$ INTO HEAD CG & H-III, M50 \\
\hline H3-50-61 & FRONTAL & UNPADDED IMPACTOR AT $0^{\circ}$ INTO FOREHEAD & H-III, M50 \\
\hline H3-50-57 & FRONTAL & UNPADDED IMPACTOR AT $0^{\circ}$ INTO FOREHEAD & H-III, M50 \\
\hline H3-50-25 & FRONTAL & UNPADDED IMPACTOR AT $0^{\circ}$ INTO FOREHEAD & H-III, M50 \\
\hline H3-50-45 & FRONTAL & PADDED IMPACTOR AT $0^{\circ}$ INTO FOREHEAD & H-III, M50 \\
\hline H3-50-1 & FRONTAL & PADDED IMPACTOR AT $0^{\circ}$ INTO FOREHEAD & H-III, M50 \\
\hline H3-50-17 & FRONTAL & PADDED IMPACTOR AT $0^{\circ}$ INTO FOREHEAD & H-III, M50 \\
\hline H3-50-29 & FRONTAL & PADDED IMPACTOR AT $0^{\circ}$ INTO FOREHEAD & H-III, M50 \\
\hline $\mathrm{H} 3-50-46$ & FRONTAL & UNPADDED IMPACTOR AT $0^{\circ}$ INTO HEAD CG & H-III, M50 \\
\hline H3-50-58 & FRONTAL & UNPADDED IMPACTOR AT $0^{\circ}$ INTO HEAD CG & H-III, M50 \\
\hline H3-50-6 & FRONTAL & UNPADDED IMPACTOR AT $0^{\circ}$ INTO HEAD CG & H-III, M50 \\
\hline H3-50-18 & FRONTAL & UNPADDED IMPACTOR AT $0^{\circ}$ INTO HEAD CG & H-III, M50 \\
\hline $\mathrm{H} 3-50-30$ & FRONTAL & UNPADDED IMPACTOR AT $0^{\circ}$ INTO HEAD CG & H-III, M50 \\
\hline H3-50-42 & FRONTAL & PADDED IMPACTOR AT 0 INTO HEAD CG & H-III, M50 \\
\hline H3-50-2 & FRONTAL & PADDED IMPACTOR AT $0^{\circ}$ INTO HEAD CG & H-III, M50 \\
\hline $\mathrm{H} 3-50-62$ & FRONTAL & PADDED IMPACTOR AT $0^{\circ}$ INTO HEAD CG & H-III, M50 \\
\hline H3-50-22 & FRONTAL & PADDED IMPACTOR AT $0^{\circ}$ INTO HEAD CG & H-III, M50 \\
\hline $\mathrm{H} 3-50-50$ & FRONTAL & UNPADDED IMPACTOR AT $0^{\circ}$ INTO FOREHEAD & H-III, M50 \\
\hline $\mathrm{H} 3-50-10$ & FRONTAL & UNPADDED IMPACTOR AT $0^{\circ}$ INTO FOREHEAD & H-III, M50 \\
\hline H3-50-34 & FRONTAL & UNPADDED IMPACTOR AT $0^{\circ}$ INTO FOREHEAD & H-III, M50 \\
\hline H3-50-54 & FRONTAL & PADDED IMPACTOR AT $0^{\circ}$ INTO FOREHEAD & H-III, M50 \\
\hline H3-50-14 & FRONTAL & PADDED IMPACTOR AT $0^{\circ}$ INTO FOREHEAD & H-III, M50 \\
\hline H3-50-26 & FRONTAL & PADDED IMPACTOR AT $0^{\circ}$ INTO FOREHEAD & H-III, M50 \\
\hline H3-50-38 & FRONTAL & PADDED IMPACTOR AT $0^{\circ}$ INTO FOREHEAD & H-III, M50 \\
\hline H3-50-35 & OBLIQUE & UNPADDED IMPACTOR AT $30^{\circ}$ INTO HEAD CG & H-III, M50 \\
\hline $\mathrm{H} 3-50-51$ & OBLIQUE & UNPADDED IMPACTOR AT $30^{\circ}$ INTO HEAD CG & H-III, M50 \\
\hline H3-50-11 & OBLIQUE & UNPADDED IMPACTOR AT $30^{\circ}$ INTO HEAD CG & H-III, M50 \\
\hline H3-50-19 & OBLIQUE & UNPADDED IMPACTOR AT $30^{\circ}$ INTO HEAD CG & H-III, M50 \\
\hline H3-50-3 & OBLIQUE & UNPADDED IMPACTOR AT $30^{\circ}$ INTO HEAD CG & H-III, M50 \\
\hline H3-50-27 & OBLIQUE & UNPADDED IMPACTOR AT $30^{\circ}$ INTO HEAD CG & H-III, M50 \\
\hline $\mathrm{H} 3-50-31$ & OBLIQUE & PADDED IMPACTOR AT $30^{\circ}$ INTO HEAD CG & H-III, M50 \\
\hline H3-50-39 & OBLIQUE & PADDED IMPACTOR AT $30^{\circ}$ INTO HEAD CG & H-III, M50 \\
\hline H3-50-55 & OBLIQUE & PADDED IMPACTOR AT $30^{\circ}$ INTO HEAD CG & H-III, M50 \\
\hline H3-50-43 & OBLIQUE & PADDED IMPACTOR AT $30^{\circ}$ INTO HEAD CG & H-III, M50 \\
\hline H3-50-47 & OBLIQUE & PADDED IMPACTOR AT $30^{\circ}$ INTO HEAD CG & H-III, M50 \\
\hline H3-50-15 & OBLIQUE & PADDED IMPACTOR AT $30^{\circ}$ INTO HEAD CG & H-III, M50 \\
\hline H3-50-7 & OBLIQUE & PADDED IMPACTOR AT $30^{\circ}$ INTO HEAD CG & H-III, M50 \\
\hline H3-50-23 & OBLIQUE & PADDED IMPACTOR AT 30 INTO HEAD CG & H-III, M50 \\
\hline H3-50-59 & FRONTAL & UNPADDED IMPACTOR AT $0^{\circ}$ INTO FOREHEAD & H-III, M50 \\
\hline H3-50-63 & FRONTAL & PADDED IMPACTOR AT 0 INTO FOREHEAD & H-III, M50 \\
\hline $\mathrm{H} 3-50-4$ & FRONTAL & UNPADDED IMPACTOR AT $0^{\circ}$ INTO FOREHEAD & H-III, M50 \\
\hline H3-50-16 & FRONTAL & UNPADDED IMPACTOR AT $0^{\circ}$ INTO FOREHEAD & H-III, M50 \\
\hline H3-50-8 & FRONTAL & UNPADDED IMPACTOR AT $0^{\circ}$ INTO FOREHEAD & H-III, M50 \\
\hline
\end{tabular}




\begin{tabular}{|c|c|c|c|}
\hline H3-50-12 & FRONTAL & UNPADDED IMPACTOR AT $0^{\circ}$ INTO FOREHEAD & H-III, M50 \\
\hline H3-50-20 & FRONTAL & UNPADDED IMPACTOR AT $0^{\circ}$ INTO FOREHEAD & H-III, M50 \\
\hline H3-50-24 & FRONTAL & UNPADDED IMPACTOR AT $0^{\circ}$ INTO FOREHEAD & H-III, M50 \\
\hline H3-50-28 & FRONTAL & UNPADDED IMPACTOR AT $0^{\circ}$ INTO FOREHEAD & H-III, M50 \\
\hline H3-50-40 & FRONTAL & UNPADDED IMPACTOR AT $0^{\circ}$ INTO FOREHEAD & H-III, M50 \\
\hline H3-50-32 & FRONTAL & UNPADDED IMPACTOR AT $0^{\circ}$ INTO FOREHEAD & H-III, M50 \\
\hline H3-50-36 & FRONTAL & UNPADDED IMPACTOR AT $0^{\circ}$ INTO FOREHEAD & H-III, M50 \\
\hline H3-50-52 & FRONTAL & UNPADDED IMPACTOR AT $0^{\circ}$ INTO FOREHEAD & H-III, M50 \\
\hline H3-50-44 & FRONTAL & UNPADDED IMPACTOR AT $0^{\circ}$ INTO FOREHEAD & H-III, M50 \\
\hline H3-50-48 & FRONTAL & UNPADDED IMPACTOR AT $0^{\circ}$ INTO FOREHEAD & H-III, M50 \\
\hline H3-50-56 & FRONTAL & UNPADDED IMPACTOR AT $0^{\circ}$ INTO FOREHEAD & H-III, M50 \\
\hline $\mathrm{H} 3-50-60$ & FRONTAL & PADDED IMPACTOR AT $0^{\circ}$ INTO FOREHEAD & H-III, M50 \\
\hline
\end{tabular}

\section{J.4 IIHS Crash Tests}

\begin{tabular}{|c|c|c|c|c|}
\hline $\begin{array}{c}\text { Source } \\
\text { test \# }\end{array}$ & $\begin{array}{l}\text { Occ. } \\
\text { Loc. }\end{array}$ & $\begin{array}{c}\text { Impact } \\
\text { condition }\end{array}$ & Test details & Surrogate \\
\hline CEN1219 & 01 & FRONTAL & VEHICLE INTO BARRIER $(0,25)$, SO & H-III, M50 \\
\hline CEN1220 & 01 & FRONTAL & VEHICLE INTO BARRIER $(0,25)$, SO & H-III, M50 \\
\hline CEN1221 & 01 & FRONTAL & VEHICLE INTO BARRIER $(0,25)$, SO & H-III, M50 \\
\hline CEN1222 & 01 & FRONTAL & VEHICLE INTO BARRIER $(0,25)$, SO & H-III, M50 \\
\hline CEN1223 & 01 & FRONTAL & VEHICLE INTO BARRIER $(0,25)$, SO & H-III, M50 \\
\hline CEN1224 & 01 & FRONTAL & VEHICLE INTO BARRIER $(0,25)$, SO & H-III, M50 \\
\hline CEN1225 & 01 & FRONTAL & VEHICLE INTO BARRIER $(0,25)$, SO & H-III, M50 \\
\hline CEN1226 & 01 & FRONTAL & VEHICLE INTO BARRIER $(0,25)$, SO & H-III, M50 \\
\hline CEN1227 & 01 & FRONTAL & VEHICLE INTO BARRIER $(0,25)$, SO & H-III, M50 \\
\hline CEN1228 & 01 & FRONTAL & VEHICLE INTO BARRIER $(0,25)$, SO & H-III, M50 \\
\hline CEN1229 & 01 & FRONTAL & VEHICLE INTO BARRIER $(0,25)$, SO & H-III, M50 \\
\hline CEN1230 & 01 & FRONTAL & VEHICLE INTO BARRIER $(0,25)$, SO & H-III, M50 \\
\hline CEN1231 & 01 & FRONTAL & VEHICLE INTO BARRIER $(0,25)$, SO & H-III, M50 \\
\hline CEN1232 & 01 & FRONTAL & VEHICLE INTO BARRIER $(0,25)$, SO & H-III, M50 \\
\hline CEN1233 & 01 & FRONTAL & VEHICLE INTO BARRIER $(0,25)$, SO & H-III, M50 \\
\hline CEN1234 & 01 & FRONTAL & VEHICLE INTO BARRIER $(0,25)$, SO & H-III, M50 \\
\hline CEN1235 & 01 & FRONTAL & VEHICLE INTO BARRIER $(0,25)$, SO & H-III, M50 \\
\hline CEN1236 & 01 & FRONTAL & VEHICLE INTO BARRIER $(0,25)$, SO & H-III, M50 \\
\hline CEN1237 & 01 & FRONTAL & VEHICLE INTO BARRIER $(0,25)$, SO & H-III, M50 \\
\hline CEN1301 & 01 & FRONTAL & VEHICLE INTO BARRIER $(0,25)$, SO & H-III, M50 \\
\hline CEN1302 & 01 & FRONTAL & VEHICLE INTO BARRIER $(0,25)$, SO & H-III, M50 \\
\hline CEN1303 & 01 & FRONTAL & VEHICLE INTO BARRIER $(0,25)$, SO & H-III, M50 \\
\hline CEN1304 & 01 & FRONTAL & VEHICLE INTO BARRIER $(0,25)$, SO & H-III, M50 \\
\hline CEN1305 & 01 & FRONTAL & VEHICLE INTO BARRIER $(0,25)$, SO & H-III, M50 \\
\hline CEN1306 & 01 & FRONTAL & VEHICLE INTO BARRIER $(0,25)$, SO & H-III, M50 \\
\hline CEN1307 & 01 & FRONTAL & VEHICLE INTO BARRIER $(0,25)$, SO & H-III, M50 \\
\hline CEN1308 & 01 & FRONTAL & VEHICLE INTO BARRIER $(0,25)$, SO & H-III, M50 \\
\hline CEN1309 & 01 & FRONTAL & VEHICLE INTO BARRIER $(0,25)$, SO & H-III, M50 \\
\hline CEN1310 & 01 & FRONTAL & VEHICLE INTO BARRIER $(0,25)$, SO & H-III, M50 \\
\hline CEN1311 & 01 & FRONTAL & VEHICLE INTO BARRIER $(0,25)$, SO & H-III, M50 \\
\hline CEN1312 & 01 & FRONTAL & VEHICLE INTO BARRIER $(0,25)$, SO & H-III, M50 \\
\hline CEN1313 & 01 & FRONTAL & VEHICLE INTO BARRIER $(0,25)$, SO & H-III, M50 \\
\hline CEN1314 & 01 & FRONTAL & VEHICLE INTO BARRIER $(0,25)$, SO & H-III, M50 \\
\hline CEN1315 & 01 & FRONTAL & VEHICLE INTO BARRIER $(0,25)$, SO & H- III, M50 \\
\hline CEN1316 & 01 & FRONTAL & VEHICLE INTO BARRIER $(0,25)$, SO & H- III, M50 \\
\hline CEN1317 & 01 & FRONTAL & VEHICLE INTO BARRIER $(0,25)$, SO & H- III, M50 \\
\hline CEN1318 & 01 & FRONTAL & VEHICLE INTO BARRIER $(0,25)$, SO & H- III, M50 \\
\hline CEN1319 & 01 & FRONTAL & VEHICLE INTO BARRIER $(0,25)$, SO & H- III, M50 \\
\hline CEN1320 & 01 & FRONTAL & VEHICLE INTO BARRIER $(0,25)$, SO & H- III, M50 \\
\hline CEN1321 & 01 & FRONTAL & VEHICLE INTO BARRIER $(0,25)$, SO & H- III, M50 \\
\hline CEN1322 & 01 & FRONTAL & VEHICLE INTO BARRIER $(0,25)$, SO & H- III, M50 \\
\hline
\end{tabular}




\begin{tabular}{|c|c|c|c|c|}
\hline CEN1323 & 01 & FRONTAL & VEHICLE INTO BARRIER $(0,25)$, SO & H- III, M50 \\
\hline CEN1324 & 01 & FRONTAL & VEHICLE INTO BARRIER $(0,25)$, SO & H- III, M50 \\
\hline CEN1325 & 01 & FRONTAL & VEHICLE INTO BARRIER $(0,25)$, SO & H- III, M50 \\
\hline CEN1326 & 01 & FRONTAL & VEHICLE INTO BARRIER $(0,25)$, SO & H- III, M50 \\
\hline CEN1327 & 01 & FRONTAL & VEHICLE INTO BARRIER $(0,25)$, SO & H- III, M50 \\
\hline CEN1328 & 01 & FRONTAL & VEHICLE INTO BARRIER $(0,25)$, SO & H- III, M50 \\
\hline CEN1329 & 01 & FRONTAL & VEHICLE INTO BARRIER $(0,25)$, SO & H- III, M50 \\
\hline CEN1330 & 01 & FRONTAL & VEHICLE INTO BARRIER $(0,25)$, SO & H- III, M50 \\
\hline CEN1331 & 01 & FRONTAL & VEHICLE INTO BARRIER $(0,25)$, SO & H- III, M50 \\
\hline CEN1332 & 01 & FRONTAL & VEHICLE INTO BARRIER $(0,25)$, SO & H- III, M50 \\
\hline CEN1333 & 01 & FRONTAL & VEHICLE INTO BARRIER $(0,25)$, SO & H- III, M50 \\
\hline CEN1334 & 01 & FRONTAL & VEHICLE INTO BARRIER $(0,25)$, SO & H- III, M50 \\
\hline CEN1335 & 01 & FRONTAL & VEHICLE INTO BARRIER $(0,25)$, SO & H- III, M50 \\
\hline CEN1336 & 01 & FRONTAL & VEHICLE INTO BARRIER $(0,25)$, SO & H- III, M50 \\
\hline CEN1337 & 01 & FRONTAL & VEHICLE INTO BARRIER $(0,25)$, SO & H- III, M50 \\
\hline CEN1338 & 01 & FRONTAL & VEHICLE INTO BARRIER $(0,25)$, SO & H- III, M50 \\
\hline CEN1339 & 01 & FRONTAL & VEHICLE INTO BARRIER $(0,25)$, SO & H- III, M50 \\
\hline CEN1340 & 01 & FRONTAL & VEHICLE INTO BARRIER $(0,25)$, SO & H- III, M50 \\
\hline CEN1341 & 01 & FRONTAL & VEHICLE INTO BARRIER $(0,25)$, SO & H- III, M50 \\
\hline CEN1342 & 01 & FRONTAL & VEHICLE INTO BARRIER $(0,25)$, SO & H- III, M50 \\
\hline CEN1343 & 01 & FRONTAL & VEHICLE INTO BARRIER $(0,25)$, SO & H- III, M50 \\
\hline CEN1344 & 01 & FRONTAL & VEHICLE INTO BARRIER $(0,25)$, SO & H- III, M50 \\
\hline CEN1345 & 01 & FRONTAL & VEHICLE INTO BARRIER $(0,25)$, SO & H- III, M50 \\
\hline CEN1346 & 01 & FRONTAL & VEHICLE INTO BARRIER $(0,25)$, SO & H- III, M50 \\
\hline CEN1347 & 01 & FRONTAL & VEHICLE INTO BARRIER $(0,25)$, SO & H- III, M50 \\
\hline CEN1348 & 01 & FRONTAL & VEHICLE INTO BARRIER $(0,25)$, SO & H- III, M50 \\
\hline CEN1349 & 01 & FRONTAL & VEHICLE INTO BARRIER $(0,25)$, SO & H- III, M50 \\
\hline CEN1401 & 01 & FRONTAL & VEHICLE INTO BARRIER $(0,25)$, SO & H- III, M50 \\
\hline CEN1402 & 01 & FRONTAL & VEHICLE INTO BARRIER $(0,25)$, SO & H- III, M50 \\
\hline CEN1403 & 01 & FRONTAL & VEHICLE INTO BARRIER $(0,25)$, SO & H- III, M50 \\
\hline CEN1404 & 01 & FRONTAL & VEHICLE INTO BARRIER $(0,25)$, SO & H- III, M50 \\
\hline CEN1405 & 01 & FRONTAL & VEHICLE INTO BARRIER $(0,25)$, SO & H- III, M50 \\
\hline CEN1406 & 01 & FRONTAL & VEHICLE INTO BARRIER $(0,25)$, SO & H- III, M50 \\
\hline CEN1407 & 01 & FRONTAL & VEHICLE INTO BARRIER $(0,25)$, SO & H- III, M50 \\
\hline CEN1408 & 01 & FRONTAL & VEHICLE INTO BARRIER $(0,25)$, SO & H- III, M50 \\
\hline CEN1409 & 01 & FRONTAL & VEHICLE INTO BARRIER $(0,25)$, SO & H- III, M50 \\
\hline CEN1410 & 01 & FRONTAL & VEHICLE INTO BARRIER $(0,25)$, SO & H- III, M50 \\
\hline CEN1411 & 01 & FRONTAL & VEHICLE INTO BARRIER $(0,25)$, SO & H- III, M50 \\
\hline CEN1412 & 01 & FRONTAL & VEHICLE INTO BARRIER $(0,25)$, SO & H- III, M50 \\
\hline CEN1413 & 01 & FRONTAL & VEHICLE INTO BARRIER $(0,25)$, SO & H- III, M50 \\
\hline CEN1414 & 01 & FRONTAL & VEHICLE INTO BARRIER $(0,25)$, SO & H- III, M50 \\
\hline CEN1415 & 01 & FRONTAL & VEHICLE INTO BARRIER $(0,25)$, SO & H- III, M50 \\
\hline CEN1416 & 01 & FRONTAL & VEHICLE INTO BARRIER $(0,25)$, SO & H- III, M50 \\
\hline CEN1418 & 01 & FRONTAL & VEHICLE INTO BARRIER $(0,25)$, SO & H- III, M50 \\
\hline CEN1419 & 01 & FRONTAL & VEHICLE INTO BARRIER $(0,25)$, SO & H- III, M50 \\
\hline CEN1420 & 01 & FRONTAL & VEHICLE INTO BARRIER $(0,25)$, SO & H- III, M50 \\
\hline CEN1421 & 01 & FRONTAL & VEHICLE INTO BARRIER $(0,25)$, SO & H- III, M50 \\
\hline CEN1422 & 01 & FRONTAL & VEHICLE INTO BARRIER $(0,25)$, SO & H- III, M50 \\
\hline CEN1423 & 01 & FRONTAL & VEHICLE INTO BARRIER $(0,25)$, SO & H- III, M50 \\
\hline CEN1424 & 01 & FRONTAL & VEHICLE INTO BARRIER $(0,25)$, SO & H- III, M50 \\
\hline CEN1425 & 01 & FRONTAL & VEHICLE INTO BARRIER $(0,25)$, SO & H- III, M50 \\
\hline CEN1426 & 01 & FRONTAL & VEHICLE INTO BARRIER $(0,25)$, SO & H- III, M50 \\
\hline CEN1427 & 01 & FRONTAL & VEHICLE INTO BARRIER $(0,25)$, SO & H- III, M50 \\
\hline CEN1428 & 01 & FRONTAL & VEHICLE INTO BARRIER $(0,25)$, SO & H- III, M50 \\
\hline CEN1430 & 01 & FRONTAL & VEHICLE INTO BARRIER $(0,25)$, SO & H- III, M50 \\
\hline CEN1432 & 01 & FRONTAL & VEHICLE INTO BARRIER $(0,25)$, SO & H- III, M50 \\
\hline CEN1433 & 01 & FRONTAL & VEHICLE INTO BARRIER $(0,25)$, SO & H- III, M50 \\
\hline CEN1429 & 01 & FRONTAL & VEHICLE INTO BARRIER $(0,25)$, SO & H- III, M50 \\
\hline CEN1431 & 01 & FRONTAL & VEHICLE INTO BARRIER $(0,25)$, SO & H- III, M50 \\
\hline CEN1434 & 01 & FRONTAL & VEHICLE INTO BARRIER $(0,25)$, SO & H- III, M50 \\
\hline CEN1435 & 01 & FRONTAL & VEHICLE INTO BARRIER $(0,25)$, SO & H- III, M50 \\
\hline CEN1436 & 01 & FRONTAL & VEHICLE INTO BARRIER $(0,25)$, SO & H- III, M50 \\
\hline
\end{tabular}




\begin{tabular}{|c|c|c|c|c|}
\hline CEN1437 & 01 & FRONTAL & VEHICLE INTO BARRIER $(0,25)$, SO & H- III, M50 \\
\hline CEN1438 & 01 & FRONTAL & VEHICLE INTO BARRIER $(0,25)$, SO & H- III, M50 \\
\hline CEN1439 & 01 & FRONTAL & VEHICLE INTO BARRIER $(0,25)$, SO & H- III, M50 \\
\hline CEN1440 & 01 & FRONTAL & VEHICLE INTO BARRIER $(0,25)$, SO & H- III, M50 \\
\hline CEN1442 & 01 & FRONTAL & VEHICLE INTO BARRIER $(0,25)$, SO & H- III, M50 \\
\hline CEN1443 & 01 & FRONTAL & VEHICLE INTO BARRIER $(0,25)$, SO & H- III, M50 \\
\hline CEN1444 & 01 & FRONTAL & VEHICLE INTO BARRIER $(0,25)$, SO & H- III, M50 \\
\hline CEN1445 & 01 & FRONTAL & VEHICLE INTO BARRIER $(0,25)$, SO & H- III, M50 \\
\hline CEN1446 & 01 & FRONTAL & VEHICLE INTO BARRIER $(0,25)$, SO & H- III, M50 \\
\hline CEN1447 & 01 & FRONTAL & VEHICLE INTO BARRIER $(0,25)$, SO & H- III, M50 \\
\hline CEN1448 & 01 & FRONTAL & VEHICLE INTO BARRIER $(0,25)$, SO & H- III, M50 \\
\hline CEN1449 & 01 & FRONTAL & VEHICLE INTO BARRIER $(0,25)$, SO & H- III, M50 \\
\hline CEN1450 & 01 & FRONTAL & VEHICLE INTO BARRIER $(0,25)$, SO & H- III, M50 \\
\hline CEN1452 & 01 & FRONTAL & VEHICLE INTO BARRIER $(0,25)$, SO & H- III, M50 \\
\hline CEN1453 & 01 & FRONTAL & VEHICLE INTO BARRIER $(0,25)$, SO & H- III, M50 \\
\hline CEN1501 & 01 & FRONTAL & VEHICLE INTO BARRIER $(0,25)$, SO & H- III, M50 \\
\hline CEN1502 & 01 & FRONTAL & VEHICLE INTO BARRIER $(0,25)$, SO & H- III, M50 \\
\hline CEN1503 & 01 & FRONTAL & VEHICLE INTO BARRIER $(0,25)$, SO & H- III, M50 \\
\hline CEN1504 & 01 & FRONTAL & VEHICLE INTO BARRIER $(0,25)$, SO & H- III, M50 \\
\hline CEN1505 & 01 & FRONTAL & VEHICLE INTO BARRIER $(0,25)$, SO & H- III, M50 \\
\hline CEN1506 & 01 & FRONTAL & VEHICLE INTO BARRIER $(0,25)$, SO & H- III, M50 \\
\hline CEN1507 & 01 & FRONTAL & VEHICLE INTO BARRIER $(0,25)$, SO & H- III, M50 \\
\hline CEN1508 & 01 & FRONTAL & VEHICLE INTO BARRIER $(0,25)$, SO & H- III, M50 \\
\hline CEN1509 & 01 & FRONTAL & VEHICLE INTO BARRIER $(0,25)$, SO & H- III, M50 \\
\hline CEN1510 & 01 & FRONTAL & VEHICLE INTO BARRIER $(0,25)$, SO & H- III, M50 \\
\hline CEN1511 & 01 & FRONTAL & VEHICLE INTO BARRIER $(0,25)$, SO & H- III, M50 \\
\hline CEN1512 & 01 & FRONTAL & VEHICLE INTO BARRIER $(0,25)$, SO & H- III, M50 \\
\hline CEN1514 & 01 & FRONTAL & VEHICLE INTO BARRIER $(0,25)$, SO & H- III, M50 \\
\hline CEN1515 & 01 & FRONTAL & VEHICLE INTO BARRIER $(0,25)$, SO & H- III, M50 \\
\hline CEN1518 & 01 & FRONTAL & VEHICLE INTO BARRIER $(0,25)$, SO & H- III, M50 \\
\hline CEN1539 & 01 & FRONTAL & VEHICLE INTO BARRIER $(0,25)$, SO & H- III, M50 \\
\hline CEF1206 & 01 & FRONTAL & VEHICLE INTO BARRIER $(0,40)$, MO & H- III, M50 \\
\hline CEF1207 & 01 & FRONTAL & VEHICLE INTO BARRIER $(0,40)$, MO & H- III, M50 \\
\hline CEF1208 & 01 & FRONTAL & VEHICLE INTO BARRIER $(0,40)$, MO & H- III, M50 \\
\hline CEF1301 & 01 & FRONTAL & VEHICLE INTO BARRIER $(0,40)$, MO & H- III, M50 \\
\hline CEF1302 & 01 & FRONTAL & VEHICLE INTO BARRIER $(0,40)$, MO & H- III, M50 \\
\hline CEF1303 & 01 & FRONTAL & VEHICLE INTO BARRIER $(0,40)$, MO & H- III, M50 \\
\hline CEF1304 & 01 & FRONTAL & VEHICLE INTO BARRIER $(0,40)$, MO & H- III, M50 \\
\hline CEF1305 & 01 & FRONTAL & VEHICLE INTO BARRIER $(0,40)$, MO & H- III, M50 \\
\hline CEF1306 & 01 & FRONTAL & VEHICLE INTO BARRIER $(0,40)$, MO & H- III, M50 \\
\hline CEF1307 & 01 & FRONTAL & VEHICLE INTO BARRIER $(0,40)$, MO & H- III, M50 \\
\hline CEF1308 & 01 & FRONTAL & VEHICLE INTO BARRIER $(0,40)$, MO & H- III, M50 \\
\hline CEF1402 & 01 & FRONTAL & VEHICLE INTO BARRIER $(0,40)$, MO & H- III, M50 \\
\hline CEF1403 & 01 & FRONTAL & VEHICLE INTO BARRIER $(0,40)$, MO & H- III, M50 \\
\hline CEF1404 & 01 & FRONTAL & VEHICLE INTO BARRIER $(0,40)$, MO & H- III, M50 \\
\hline CEF1405 & 01 & FRONTAL & VEHICLE INTO BARRIER $(0,40)$, MO & H- III, M50 \\
\hline CEF1406 & 01 & FRONTAL & VEHICLE INTO BARRIER $(0,40)$, MO & H- III, M50 \\
\hline CEF1501 & 01 & FRONTAL & VEHICLE INTO BARRIER $(0,40)$, MO & H- III, M50 \\
\hline *CES1308 & 01 & SIDE & SIDE IMPACT, 1500 KG MDB & SID-IIS, F05 \\
\hline *CES1309 & 01 & SIDE & SIDE IMPACT, 1500 KG MDB & SID-IIS, F05 \\
\hline *CES1310 & 01 & SIDE & SIDE IMPACT, $1500 \mathrm{KG}$ MDB & SID-IIS, F05 \\
\hline *CES1311 & 01 & SIDE & SIDE IMPACT, $1500 \mathrm{KG}$ MDB & SID-IIS, F05 \\
\hline *CES1401 & 01 & SIDE & SIDE IMPACT, 1500 KG MDB & SID-IIS, F05 \\
\hline *CES1402 & 01 & SIDE & SIDE IMPACT, 1500 KG MDB & SID-IIS, F05 \\
\hline *CES1403 & 01 & SIDE & SIDE IMPACT, $1500 \mathrm{KG}$ MDB & SID-IIS, F05 \\
\hline *CES1404 & 01 & SIDE & SIDE IMPACT, $1500 \mathrm{KG}$ MDB & SID-IIS, F05 \\
\hline *CES1405 & 01 & SIDE & SIDE IMPACT, $1500 \mathrm{KG}$ MDB & SID-IIS, F05 \\
\hline *CES1406 & 01 & SIDE & SIDE IMPACT, 1500 KG MDB & SID-IIS, F05 \\
\hline *CES1502 & 01 & SIDE & SIDE IMPACT, $1500 \mathrm{KG}$ MDB & SID-IIS, F05 \\
\hline *CES1308 & 04 & SIDE & SIDE IMPACT, 1500 KG MDB & SID-IIS, F05 \\
\hline *CES1309 & 04 & SIDE & SIDE IMPACT, 1500 KG MDB & SID-IIS, F05 \\
\hline *CES1310 & 04 & SIDE & SIDE IMPACT, $1500 \mathrm{KG}$ MDB & SID-IIS, F05 \\
\hline
\end{tabular}




\begin{tabular}{cccll}
$*$ CES1311 & 04 & SIDE & SIDE IMPACT, 1500 KG MDB & SID-IIS, F05 \\
$*$ CES1401 & 04 & SIDE & SIDE IMPACT, 1500 KG MDB & SID-IIS, F05 \\
$*$ CES1402 & 04 & SIDE & SIDE IMPACT, 1500 KG MDB & SID-IIS, F05 \\
$*$ CES1403 & 04 & SIDE & SIDE IMPACT, 1500 KG MDB & SID-IIS, F05 \\
$*$ CES1404 & 04 & SIDE & SIDE IMPACT, 1500 KG MDB & SID-IIS, F05 \\
$*$ CES1406 & 04 & SIDE & SIDE IMPACT, 1500 KG MDB & SID-IIS, F05 \\
$*$ CES1502 & 04 & SIDE & SIDE IMPACT, 1500 KG MDB & SID-IIS, F05 \\
\hline
\end{tabular}

Occupant Seat Location (Occ. Loc.): 01 = left front, $02=$ right front, 03 = right rear, $04=$ left rear

(\#\#, \#\#) indicates impact parameters (degree offset, percent overlap)

*Kinematics scaled to 50TH male using the equal-stress-equal-velocity scaling technique.

\section{J.5 Biokinetics Helmet Impactor Tests}

\begin{tabular}{|c|c|c|c|}
\hline Source ID & $\begin{array}{c}\text { Impact } \\
\text { Condition }\end{array}$ & Test Details & Surrogate \\
\hline H01-55-A & OBLIQUE & PADDED IMPACT, OBLIQUE FACEMASK & H-III, M50 \\
\hline H01-55-AP & FRONT & PADDED IMPACT, LOWER CENTRAL FACEMASK & H-III, M50 \\
\hline H01-55-B & OBLIQUE & PADDED IMPACT, UPPER OBLIQUE FACEMASK & H-III, M50 \\
\hline $\mathrm{H} 01-55-\mathrm{C}$ & SIDE & PADDED IMPACT, SIDE OF SHELL, OBLIQUE & H-III, M50 \\
\hline H01-55-D & OBLIQUE & PADDED IMPACT, REAR BOSS OF SHELL & H-III, M50 \\
\hline $\mathrm{H} 01-55-\mathrm{F}$ & FRONT & PADDED IMPACT, FRONT OF SHELL & H-III, M50 \\
\hline $\mathrm{H} 01-55-\mathrm{R}$ & REAR & PADDED IMPACT, REAR OF SHELL & H-III, M50 \\
\hline H01-55-UT & OBLIQUE & PADDED IMPACT, SIDE OF FACEMASK & H-III, M50 \\
\hline H01-74-A & OBLIQUE & PADDED IMPACT, OBLIQUE FACEMASK & H-III, M50 \\
\hline H01-74-AP & FRONT & PADDED IMPACT, LOWER CENTRAL FACEMASK & H-III, M50 \\
\hline H01-74-B & OBLIQUE & PADDED IMPACT, UPPER OBLIQUE FACEMASK & H-III, M50 \\
\hline $\mathrm{H} 01-74-\mathrm{C}$ & SIDE & PADDED IMPACT, SIDE OF SHELL, OBLIQUE & H-III, M50 \\
\hline H01-74-D & OBLIQUE & PADDED IMPACT, REAR BOSS OF SHELL & H-III, M50 \\
\hline $\mathrm{H} 01-74-\mathrm{F}$ & FRONT & PADDED IMPACT, FRONT OF SHELL & H-III, M50 \\
\hline H01-74-R & REAR & PADDED IMPACT, REAR OF SHELL & H-III, M50 \\
\hline H01-74-UT & OBLIQUE & PADDED IMPACT, SIDE OF FACEMASK & H-III, M50 \\
\hline H01-93-A & OBLIQUE & PADDED IMPACT, OBLIQUE FACEMASK & H-III, M50 \\
\hline H01-93-AP & FRONT & PADDED IMPACT, LOWER CENTRAL FACEMASK & H-III, M50 \\
\hline H01-93-B & OBLIQUE & PADDED IMPACT, UPPER OBLIQUE FACEMASK & H-III, M50 \\
\hline $\mathrm{H} 01-93-\mathrm{C}$ & SIDE & PADDED IMPACT, SIDE OF SHELL, OBLIQUE & H-III, M50 \\
\hline H01-93-D & OBLIQUE & PADDED IMPACT, REAR BOSS OF SHELL & H-III, M50 \\
\hline H01-93-F & FRONT & PADDED IMPACT, FRONT OF SHELL & H-III, M50 \\
\hline H01-93-R & REAR & PADDED IMPACT, REAR OF SHELL & H-III, M50 \\
\hline H01-93-UT & OBLIQUE & PADDED IMPACT, SIDE OF FACEMASK & H-III, M50 \\
\hline $\mathrm{H} 02-55-\mathrm{A}$ & OBLIQUE & PADDED IMPACT, OBLIQUE FACEMASK & H-III, M50 \\
\hline H02-55-AP & FRONT & PADDED IMPACT, LOWER CENTRAL FACEMASK & H-III, M50 \\
\hline H02-55-B & OBLIQUE & PADDED IMPACT, UPPER OBLIQUE FACEMASK & H-III, M50 \\
\hline $\mathrm{H} 02-55-\mathrm{C}$ & SIDE & PADDED IMPACT, SIDE OF SHELL, OBLIQUE & H-III, M50 \\
\hline H02-55-D & OBLIQUE & PADDED IMPACT, REAR BOSS OF SHELL & H-III, M50 \\
\hline $\mathrm{H} 02-55-\mathrm{F}$ & FRONT & PADDED IMPACT, FRONT OF SHELL & H-III, M50 \\
\hline $\mathrm{H} 02-55-\mathrm{R}$ & REAR & PADDED IMPACT, REAR OF SHELL & H-III, M50 \\
\hline H02-55-UT & OBLIQUE & PADDED IMPACT, SIDE OF FACEMASK & H-III, M50 \\
\hline $\mathrm{H} 02-74-\mathrm{A}$ & OBLIQUE & PADDED IMPACT, OBLIQUE FACEMASK & H-III, M50 \\
\hline H02-74-AP & FRONT & PADDED IMPACT, LOWER CENTRAL FACEMASK & H-III, M50 \\
\hline H02-74-B & OBLIQUE & PADDED IMPACT, UPPER OBLIQUE FACEMASK & H-III, M50 \\
\hline $\mathrm{H} 02-74-\mathrm{C}$ & SIDE & PADDED IMPACT, SIDE OF SHELL, OBLIQUE & H-III, M50 \\
\hline H02-74-D & OBLIQUE & PADDED IMPACT, REAR BOSS OF SHELL & H-III, M50 \\
\hline $\mathrm{H} 02-74-\mathrm{F}$ & FRONT & PADDED IMPACT, FRONT OF SHELL & H-III, M50 \\
\hline H02-74-R & REAR & PADDED IMPACT, REAR OF SHELL & H-III, M50 \\
\hline H02-74-UT & OBLIQUE & PADDED IMPACT, SIDE OF FACEMASK & H-III, M50 \\
\hline $\mathrm{H} 02-93-\mathrm{A}$ & OBLIQUE & PADDED IMPACT, OBLIQUE FACEMASK & H-III, M50 \\
\hline H02-93-AP & FRONT & PADDED IMPACT, LOWER CENTRAL FACEMASK & H-III, M50 \\
\hline H02-93-B & OBLIQUE & PADDED IMPACT, UPPER OBLIQUE FACEMASK & H-III, M50 \\
\hline
\end{tabular}




\begin{tabular}{|c|c|c|c|}
\hline $\mathrm{H} 02-93-\mathrm{C}$ & SIDE & PADDED IMPACT, SIDE OF SHELL, OBLIQUE & H-III, M50 \\
\hline H02-93-D & OBLIQUE & PADDED IMPACT, REAR BOSS OF SHELL & H-III, M50 \\
\hline H02-93-F & FRONT & PADDED IMPACT, FRONT OF SHELL & H-III, M50 \\
\hline H02-93-R & REAR & PADDED IMPACT, REAR OF SHELL & H-III, M50 \\
\hline H02-93-UT & OBLIQUE & PADDED IMPACT, SIDE OF FACEMASK & H-III, M50 \\
\hline H03-55-A & OBLIQUE & PADDED IMPACT, OBLIQUE FACEMASK & H-III, M50 \\
\hline H03-55-AP & FRONT & PADDED IMPACT, LOWER CENTRAL FACEMASK & H-III, M50 \\
\hline H03-55-B & OBLIQUE & PADDED IMPACT, UPPER OBLIQUE FACEMASK & H-III, M50 \\
\hline $\mathrm{H} 03-55-\mathrm{C}$ & SIDE & PADDED IMPACT, SIDE OF SHELL, OBLIQUE & H-III, M50 \\
\hline H03-55-D & OBLIQUE & PADDED IMPACT, REAR BOSS OF SHELL & H-III, M50 \\
\hline $\mathrm{H} 03-55-\mathrm{F}$ & FRONT & PADDED IMPACT, FRONT OF SHELL & H-III, M50 \\
\hline H03-55-R & REAR & PADDED IMPACT, REAR OF SHELL & H-III, M50 \\
\hline H03-55-UT & OBLIQUE & PADDED IMPACT, SIDE OF FACEMASK & H-III, M50 \\
\hline H03-74-A & OBLIQUE & PADDED IMPACT, OBLIQUE FACEMASK & H-III, M50 \\
\hline H03-74-AP & FRONT & PADDED IMPACT, LOWER CENTRAL FACEMASK & H-III, M50 \\
\hline H03-74-B & OBLIQUE & PADDED IMPACT, UPPER OBLIQUE FACEMASK & H-III, M50 \\
\hline $\mathrm{H} 03-74-\mathrm{C}$ & SIDE & PADDED IMPACT, SIDE OF SHELL, OBLIQUE & H-III, M50 \\
\hline H03-74-D & OBLIQUE & PADDED IMPACT, REAR BOSS OF SHELL & H-III, M50 \\
\hline H03-74-F & FRONT & PADDED IMPACT, FRONT OF SHELL & H-III, M50 \\
\hline H03-74-R & REAR & PADDED IMPACT, REAR OF SHELL & H-III, M50 \\
\hline H03-74-UT & OBLIQUE & PADDED IMPACT, SIDE OF FACEMASK & H-III, M50 \\
\hline H03-93-A & OBLIQUE & PADDED IMPACT, OBLIQUE FACEMASK & H-III, M50 \\
\hline H03-93-AP & FRONT & PADDED IMPACT, LOWER CENTRAL FACEMASK & H-III, M50 \\
\hline H03-93-B & OBLIQUE & PADDED IMPACT, UPPER OBLIQUE FACEMASK & H-III, M50 \\
\hline $\mathrm{H} 03-93-\mathrm{C}$ & SIDE & PADDED IMPACT, SIDE OF SHELL, OBLIQUE & H-III, M50 \\
\hline H03-93-D & OBLIQUE & PADDED IMPACT, REAR BOSS OF SHELL & H-III, M50 \\
\hline H03-93-F & FRONT & PADDED IMPACT, FRONT OF SHELL & H-III, M50 \\
\hline H03-93-R & REAR & PADDED IMPACT, REAR OF SHELL & H-III, M50 \\
\hline H03-93-UT & OBLIQUE & PADDED IMPACT, SIDE OF FACEMASK & H-III, M50 \\
\hline H04-55-A & OBLIQUE & PADDED IMPACT, OBLIQUE FACEMASK & H-III, M50 \\
\hline H04-55-AP & FRONT & PADDED IMPACT, LOWER CENTRAL FACEMASK & H-III, M50 \\
\hline H04-55-B & OBLIQUE & PADDED IMPACT, UPPER OBLIQUE FACEMASK & H-III, M50 \\
\hline $\mathrm{H} 04-55-\mathrm{C}$ & SIDE & PADDED IMPACT, SIDE OF SHELL, OBLIQUE & H-III, M50 \\
\hline H04-55-D & OBLIQUE & PADDED IMPACT, REAR BOSS OF SHELL & H-III, M50 \\
\hline $\mathrm{H} 04-55-\mathrm{F}$ & FRONT & PADDED IMPACT, FRONT OF SHELL & H-III, M50 \\
\hline H04-55-R & REAR & PADDED IMPACT, REAR OF SHELL & H-III, M50 \\
\hline H04-55-UT & OBLIQUE & PADDED IMPACT, SIDE OF FACEMASK & H-III, M50 \\
\hline H04-74-A & OBLIQUE & PADDED IMPACT, OBLIQUE FACEMASK & H-III, M50 \\
\hline H04-74-AP & FRONT & PADDED IMPACT, LOWER CENTRAL FACEMASK & H-III, M50 \\
\hline H04-74-B & OBLIQUE & PADDED IMPACT, UPPER OBLIQUE FACEMASK & H-III, M50 \\
\hline $\mathrm{H} 04-74-\mathrm{C}$ & SIDE & PADDED IMPACT, SIDE OF SHELL, OBLIQUE & H-III, M50 \\
\hline H04-74-D & OBLIQUE & PADDED IMPACT, REAR BOSS OF SHELL & H-III, M50 \\
\hline $\mathrm{H} 04-74-\mathrm{F}$ & FRONT & PADDED IMPACT, FRONT OF SHELL & H-III, M50 \\
\hline H04-74-R & REAR & PADDED IMPACT, REAR OF SHELL & H-III, M50 \\
\hline H04-74-UT & OBLIQUE & PADDED IMPACT, SIDE OF FACEMASK & H-III, M50 \\
\hline H04-93-A & OBLIQUE & PADDED IMPACT, OBLIQUE FACEMASK & H-III, M50 \\
\hline H04-93-AP & FRONT & PADDED IMPACT, LOWER CENTRAL FACEMASK & H-III, M50 \\
\hline H04-93-B & OBLIQUE & PADDED IMPACT, UPPER OBLIQUE FACEMASK & H-III, M50 \\
\hline $\mathrm{H} 04-93-\mathrm{C}$ & SIDE & PADDED IMPACT, SIDE OF SHELL, OBLIQUE & H-III, M50 \\
\hline H04-93-D & OBLIQUE & PADDED IMPACT, REAR BOSS OF SHELL & H-III, M50 \\
\hline H04-93-F & FRONT & PADDED IMPACT, FRONT OF SHELL & H-III, M50 \\
\hline H04-93-R & REAR & PADDED IMPACT, REAR OF SHELL & H-III, M50 \\
\hline H04-93-UT & OBLIQUE & PADDED IMPACT, SIDE OF FACEMASK & H-III, M50 \\
\hline H05-55-A & OBLIQUE & PADDED IMPACT, OBLIQUE FACEMASK & H-III, M50 \\
\hline H05-55-AP & FRONT & PADDED IMPACT, LOWER CENTRAL FACEMASK & H-III, M50 \\
\hline H05-55-B & OBLIQUE & PADDED IMPACT, UPPER OBLIQUE FACEMASK & H-III, M50 \\
\hline $\mathrm{H} 05-55-\mathrm{C}$ & SIDE & PADDED IMPACT, SIDE OF SHELL, OBLIQUE & H-III, M50 \\
\hline H05-55-D & OBLIQUE & PADDED IMPACT, REAR BOSS OF SHELL & H-III, M50 \\
\hline $\mathrm{H} 05-55-\mathrm{F}$ & FRONT & PADDED IMPACT, FRONT OF SHELL & H-III, M50 \\
\hline H05-55-R & REAR & PADDED IMPACT, REAR OF SHELL & H-III, M50 \\
\hline H05-55-UT & OBLIQUE & PADDED IMPACT, SIDE OF FACEMASK & H-III, M50 \\
\hline H05-74-A & OBLIQUE & PADDED IMPACT, OBLIQUE FACEMASK & H-III, M50 \\
\hline
\end{tabular}




\begin{tabular}{|c|c|c|c|}
\hline H05-74-AP & FRONT & PADDED IMPACT, LOWER CENTRAL FACEMASK & H-III, M50 \\
\hline H05-74-B & OBLIQUE & PADDED IMPACT, UPPER OBLIQUE FACEMASK & H-III, M50 \\
\hline $\mathrm{H} 05-74-\mathrm{C}$ & SIDE & PADDED IMPACT, SIDE OF SHELL, OBLIQUE & H-III, M50 \\
\hline H05-74-D & OBLIQUE & PADDED IMPACT, REAR BOSS OF SHELL & H-III, M50 \\
\hline H05-74-F & FRONT & PADDED IMPACT, FRONT OF SHELL & H-III, M50 \\
\hline H05-74-R & REAR & PADDED IMPACT, REAR OF SHELL & H-III, M50 \\
\hline H05-74-UT & OBLIQUE & PADDED IMPACT, SIDE OF FACEMASK & H-III, M50 \\
\hline H05-93-A & OBLIQUE & PADDED IMPACT, OBLIQUE FACEMASK & H-III, M50 \\
\hline H05-93-AP & FRONT & PADDED IMPACT, LOWER CENTRAL FACEMASK & H-III, M50 \\
\hline H05-93-B & OBLIQUE & PADDED IMPACT, UPPER OBLIQUE FACEMASK & H-III, M50 \\
\hline $\mathrm{H} 05-93-\mathrm{C}$ & SIDE & PADDED IMPACT, SIDE OF SHELL, OBLIQUE & H-III, M50 \\
\hline H05-93-D & OBLIQUE & PADDED IMPACT, REAR BOSS OF SHELL & H-III, M50 \\
\hline H05-93-F & FRONT & PADDED IMPACT, FRONT OF SHELL & H-III, M50 \\
\hline H05-93-R & REAR & PADDED IMPACT, REAR OF SHELL & H-III, M50 \\
\hline H05-93-UT & OBLIQUE & PADDED IMPACT, SIDE OF FACEMASK & H-III, M50 \\
\hline H06-55-A & OBLIQUE & PADDED IMPACT, OBLIQUE FACEMASK & H-III, M50 \\
\hline H06-55-AP & FRONT & PADDED IMPACT, LOWER CENTRAL FACEMASK & H-III, M50 \\
\hline H06-55-B & OBLIQUE & PADDED IMPACT, UPPER OBLIQUE FACEMASK & H-III, M50 \\
\hline H06-55-C & SIDE & PADDED IMPACT, SIDE OF SHELL, OBLIQUE & H-III, M50 \\
\hline H06-55-D & OBLIQUE & PADDED IMPACT, REAR BOSS OF SHELL & H-III, M50 \\
\hline $\mathrm{H} 06-55-\mathrm{F}$ & FRONT & PADDED IMPACT, FRONT OF SHELL & H-III, M50 \\
\hline H06-55-R & REAR & PADDED IMPACT, REAR OF SHELL & H-III, M50 \\
\hline H06-55-UT & OBLIQUE & PADDED IMPACT, SIDE OF FACEMASK & H-III, M50 \\
\hline H06-74-A & OBLIQUE & PADDED IMPACT, OBLIQUE FACEMASK & H-III, M50 \\
\hline H06-74-AP & FRONT & PADDED IMPACT, LOWER CENTRAL FACEMASK & H-III, M50 \\
\hline H06-74-B & OBLIQUE & PADDED IMPACT, UPPER OBLIQUE FACEMASK & H-III, M50 \\
\hline H06-74-C & SIDE & PADDED IMPACT, SIDE OF SHELL, OBLIQUE & H-III, M50 \\
\hline H06-74-D & OBLIQUE & PADDED IMPACT, REAR BOSS OF SHELL & H-III, M50 \\
\hline H06-74-F & FRONT & PADDED IMPACT, FRONT OF SHELL & H-III, M50 \\
\hline H06-74-R & REAR & PADDED IMPACT, REAR OF SHELL & H-III, M50 \\
\hline H06-74-UT & OBLIQUE & PADDED IMPACT, SIDE OF FACEMASK & H-III, M50 \\
\hline H06-93-A & OBLIQUE & PADDED IMPACT, OBLIQUE FACEMASK & H-III, M50 \\
\hline H06-93-AP & FRONT & PADDED IMPACT, LOWER CENTRAL FACEMASK & H-III, M50 \\
\hline H06-93-B & OBLIQUE & PADDED IMPACT, UPPER OBLIQUE FACEMASK & H-III, M50 \\
\hline H06-93-C & SIDE & PADDED IMPACT, SIDE OF SHELL, OBLIQUE & H-III, M50 \\
\hline H06-93-D & OBLIQUE & PADDED IMPACT, REAR BOSS OF SHELL & H-III, M50 \\
\hline H06-93-F & FRONT & PADDED IMPACT, FRONT OF SHELL & H-III, M50 \\
\hline H06-93-R & REAR & PADDED IMPACT, REAR OF SHELL & H-III, M50 \\
\hline H06-93-UT & OBLIQUE & PADDED IMPACT, SIDE OF FACEMASK & H-III, M50 \\
\hline H07-55-A & OBLIQUE & PADDED IMPACT, OBLIQUE FACEMASK & H-III, M50 \\
\hline H07-55-AP & FRONT & PADDED IMPACT, LOWER CENTRAL FACEMASK & H-III, M50 \\
\hline H07-55-B & OBLIQUE & PADDED IMPACT, UPPER OBLIQUE FACEMASK & H-III, M50 \\
\hline $\mathrm{H} 07-55-\mathrm{C}$ & SIDE & PADDED IMPACT, SIDE OF SHELL, OBLIQUE & H-III, M50 \\
\hline H07-55-D & OBLIQUE & PADDED IMPACT, REAR BOSS OF SHELL & H-III, M50 \\
\hline $\mathrm{H} 07-55-\mathrm{F}$ & FRONT & PADDED IMPACT, FRONT OF SHELL & H-III, M50 \\
\hline $\mathrm{H} 07-55-\mathrm{R}$ & REAR & PADDED IMPACT, REAR OF SHELL & H-III, M50 \\
\hline H07-55-UT & OBLIQUE & PADDED IMPACT, SIDE OF FACEMASK & H-III, M50 \\
\hline H07-74-A & OBLIQUE & PADDED IMPACT, OBLIQUE FACEMASK & H-III, M50 \\
\hline H07-74-AP & FRONT & PADDED IMPACT, LOWER CENTRAL FACEMASK & H-III, M50 \\
\hline H07-74-B & OBLIQUE & PADDED IMPACT, UPPER OBLIQUE FACEMASK & H-III, M50 \\
\hline $\mathrm{H} 07-74-\mathrm{C}$ & SIDE & PADDED IMPACT, SIDE OF SHELL, OBLIQUE & H-III, M50 \\
\hline H07-74-D & OBLIQUE & PADDED IMPACT, REAR BOSS OF SHELL & H-III, M50 \\
\hline $\mathrm{H} 07-74-\mathrm{F}$ & FRONT & PADDED IMPACT, FRONT OF SHELL & H-III, M50 \\
\hline H07-74-R & REAR & PADDED IMPACT, REAR OF SHELL & H-III, M50 \\
\hline H07-74-UT & OBLIQUE & PADDED IMPACT, SIDE OF FACEMASK & H-III, M50 \\
\hline H07-93-A & OBLIQUE & PADDED IMPACT, OBLIQUE FACEMASK & H-III, M50 \\
\hline H07-93-AP & FRONT & PADDED IMPACT, LOWER CENTRAL FACEMASK & H-III, M50 \\
\hline H07-93-B & OBLIQUE & PADDED IMPACT, UPPER OBLIQUE FACEMASK & H-III, M50 \\
\hline $\mathrm{H} 07-93-\mathrm{C}$ & SIDE & PADDED IMPACT, SIDE OF SHELL, OBLIQUE & H-III, M50 \\
\hline H07-93-D & OBLIQUE & PADDED IMPACT, REAR BOSS OF SHELL & H-III, M50 \\
\hline H07-93-F & FRONT & PADDED IMPACT, FRONT OF SHELL & H-III, M50 \\
\hline H07-93-R & REAR & PADDED IMPACT, REAR OF SHELL & H-III, M50 \\
\hline
\end{tabular}




\begin{tabular}{|c|c|c|c|}
\hline H07-93-UT & OBLIQUE & PADDED IMPACT, SIDE OF FACEMASK & H-III, M50 \\
\hline H08-55-A & OBLIQUE & PADDED IMPACT, OBLIQUE FACEMASK & H-III, M50 \\
\hline H08-55-AP & FRONT & PADDED IMPACT, LOWER CENTRAL FACEMASK & H-III, M50 \\
\hline H08-55-B & OBLIQUE & PADDED IMPACT, UPPER OBLIQUE FACEMASK & H-III, M50 \\
\hline $\mathrm{H} 08-55-\mathrm{C}$ & SIDE & PADDED IMPACT, SIDE OF SHELL, OBLIQUE & H-III, M50 \\
\hline H08-55-D & OBLIQUE & PADDED IMPACT, REAR BOSS OF SHELL & H-III, M50 \\
\hline $\mathrm{H} 08-55-\mathrm{F}$ & FRONT & PADDED IMPACT, FRONT OF SHELL & H-III, M50 \\
\hline H08-55-R & REAR & PADDED IMPACT, REAR OF SHELL & H-III, M50 \\
\hline H08-55-UT & OBLIQUE & PADDED IMPACT, SIDE OF FACEMASK & H-III, M50 \\
\hline H08-74-A & OBLIQUE & PADDED IMPACT, OBLIQUE FACEMASK & H-III, M50 \\
\hline H08-74-AP & FRONT & PADDED IMPACT, LOWER CENTRAL FACEMASK & H-III, M50 \\
\hline H08-74-B & OBLIQUE & PADDED IMPACT, UPPER OBLIQUE FACEMASK & H-III, M50 \\
\hline $\mathrm{H} 08-74-\mathrm{C}$ & SIDE & PADDED IMPACT, SIDE OF SHELL, OBLIQUE & H-III, M50 \\
\hline H08-74-D & OBLIQUE & PADDED IMPACT, REAR BOSS OF SHELL & H-III, M50 \\
\hline H08-74-F & FRONT & PADDED IMPACT, FRONT OF SHELL & H-III, M50 \\
\hline H08-74-R & REAR & PADDED IMPACT, REAR OF SHELL & H-III, M50 \\
\hline H08-74-UT & OBLIQUE & PADDED IMPACT, SIDE OF FACEMASK & H-III, M50 \\
\hline H08-93-A & OBLIQUE & PADDED IMPACT, OBLIQUE FACEMASK & H-III, M50 \\
\hline H08-93-AP & FRONT & PADDED IMPACT, LOWER CENTRAL FACEMASK & H-III, M50 \\
\hline H08-93-B & OBLIQUE & PADDED IMPACT, UPPER OBLIQUE FACEMASK & H-III, M50 \\
\hline $\mathrm{H} 08-93-\mathrm{C}$ & SIDE & PADDED IMPACT, SIDE OF SHELL, OBLIQUE & H-III, M50 \\
\hline H08-93-D & OBLIQUE & PADDED IMPACT, REAR BOSS OF SHELL & H-III, M50 \\
\hline H08-93-F & FRONT & PADDED IMPACT, FRONT OF SHELL & H-III, M50 \\
\hline H08-93-R & REAR & PADDED IMPACT, REAR OF SHELL & H-III, M50 \\
\hline H08-93-UT & OBLIQUE & PADDED IMPACT, SIDE OF FACEMASK & H-III, M50 \\
\hline H09-55-A & OBLIQUE & PADDED IMPACT, OBLIQUE FACEMASK & H-III, M50 \\
\hline H09-55-AP & FRONT & PADDED IMPACT, LOWER CENTRAL FACEMASK & H-III, M50 \\
\hline H09-55-B & OBLIQUE & PADDED IMPACT, UPPER OBLIQUE FACEMASK & H-III, M50 \\
\hline $\mathrm{H} 09-55-\mathrm{C}$ & SIDE & PADDED IMPACT, SIDE OF SHELL, OBLIQUE & H-III, M50 \\
\hline H09-55-D & OBLIQUE & PADDED IMPACT, REAR BOSS OF SHELL & H-III, M50 \\
\hline $\mathrm{H} 09-55-\mathrm{F}$ & FRONT & PADDED IMPACT, FRONT OF SHELL & H-III, M50 \\
\hline H09-55-R & REAR & PADDED IMPACT, REAR OF SHELL & H-III, M50 \\
\hline H09-55-UT & OBLIQUE & PADDED IMPACT, SIDE OF FACEMASK & H-III, M50 \\
\hline H09-74-A & OBLIQUE & PADDED IMPACT, OBLIQUE FACEMASK & H-III, M50 \\
\hline H09-74-AP & FRONT & PADDED IMPACT, LOWER CENTRAL FACEMASK & H-III, M50 \\
\hline H09-74-B & OBLIQUE & PADDED IMPACT, UPPER OBLIQUE FACEMASK & H-III, M50 \\
\hline $\mathrm{H} 09-74-\mathrm{C}$ & SIDE & PADDED IMPACT, SIDE OF SHELL, OBLIQUE & H-III, M50 \\
\hline H09-74-D & OBLIQUE & PADDED IMPACT, REAR BOSS OF SHELL & H-III, M50 \\
\hline H09-74-F & FRONT & PADDED IMPACT, FRONT OF SHELL & H-III, M50 \\
\hline H09-74-R & REAR & PADDED IMPACT, REAR OF SHELL & H-III, M50 \\
\hline H09-74-UT & OBLIQUE & PADDED IMPACT, SIDE OF FACEMASK & H-III, M50 \\
\hline H09-93-A & OBLIQUE & PADDED IMPACT, OBLIQUE FACEMASK & H-III, M50 \\
\hline H09-93-AP & FRONT & PADDED IMPACT, LOWER CENTRAL FACEMASK & H-III, M50 \\
\hline H09-93-B & OBLIQUE & PADDED IMPACT, UPPER OBLIQUE FACEMASK & H-III, M50 \\
\hline $\mathrm{H} 09-93-\mathrm{C}$ & SIDE & PADDED IMPACT, SIDE OF SHELL, OBLIQUE & H-III, M50 \\
\hline H09-93-D & OBLIQUE & PADDED IMPACT, REAR BOSS OF SHELL & H-III, M50 \\
\hline H09-93-F & FRONT & PADDED IMPACT, FRONT OF SHELL & H-III, M50 \\
\hline H09-93-R & REAR & PADDED IMPACT, REAR OF SHELL & H-III, M50 \\
\hline H09-93-UT & OBLIQUE & PADDED IMPACT, SIDE OF FACEMASK & H-III, M50 \\
\hline H10-55-A & OBLIQUE & PADDED IMPACT, OBLIQUE FACEMASK & H-III, M50 \\
\hline H10-55-AP & FRONT & PADDED IMPACT, LOWER CENTRAL FACEMASK & H-III, M50 \\
\hline H10-55-B & OBLIQUE & PADDED IMPACT, UPPER OBLIQUE FACEMASK & H-III, M50 \\
\hline $\mathrm{H} 10-55-\mathrm{C}$ & SIDE & PADDED IMPACT, SIDE OF SHELL, OBLIQUE & H-III, M50 \\
\hline H10-55-D & OBLIQUE & PADDED IMPACT, REAR BOSS OF SHELL & H-III, M50 \\
\hline $\mathrm{H} 10-55-\mathrm{F}$ & FRONT & PADDED IMPACT, FRONT OF SHELL & H-III, M50 \\
\hline $\mathrm{H} 10-55-\mathrm{R}$ & REAR & PADDED IMPACT, REAR OF SHELL & H-III, M50 \\
\hline H10-55-UT & OBLIQUE & PADDED IMPACT, SIDE OF FACEMASK & H-III, M50 \\
\hline H10-74-A & OBLIQUE & PADDED IMPACT, OBLIQUE FACEMASK & H-III, M50 \\
\hline H10-74-AP & FRONT & PADDED IMPACT, LOWER CENTRAL FACEMASK & H-III, M50 \\
\hline H10-74-B & OBLIQUE & PADDED IMPACT, UPPER OBLIQUE FACEMASK & H-III, M50 \\
\hline H10-74-C & SIDE & PADDED IMPACT, SIDE OF SHELL, OBLIQUE & H-III, M50 \\
\hline H10-74-D & OBLIQUE & PADDED IMPACT, REAR BOSS OF SHELL & H-III, M50 \\
\hline
\end{tabular}




\begin{tabular}{|c|c|c|c|}
\hline H10-74-F & FRONT & PADDED IMPACT, FRONT OF SHELL & H-III, M50 \\
\hline H10-74-R & REAR & PADDED IMPACT, REAR OF SHELL & H-III, M50 \\
\hline H10-74-UT & OBLIQUE & PADDED IMPACT, SIDE OF FACEMASK & H-III, M50 \\
\hline H10-93-A & OBLIQUE & PADDED IMPACT, OBLIQUE FACEMASK & H-III, M50 \\
\hline H10-93-AP & FRONT & PADDED IMPACT, LOWER CENTRAL FACEMASK & H-III, M50 \\
\hline H10-93-B & OBLIQUE & PADDED IMPACT, UPPER OBLIQUE FACEMASK & H-III, M50 \\
\hline H10-93-C & SIDE & PADDED IMPACT, SIDE OF SHELL, OBLIQUE & H-III, M50 \\
\hline H10-93-D & OBLIQUE & PADDED IMPACT, REAR BOSS OF SHELL & H-III, M50 \\
\hline $\mathrm{H} 10-93-\mathrm{F}$ & FRONT & PADDED IMPACT, FRONT OF SHELL & H-III, M50 \\
\hline H10-93-R & REAR & PADDED IMPACT, REAR OF SHELL & H-III, M50 \\
\hline H10-93-UT & OBLIQUE & PADDED IMPACT, SIDE OF FACEMASK & H-III, M50 \\
\hline H11-55-A & OBLIQUE & PADDED IMPACT, OBLIQUE FACEMASK & H-III, M50 \\
\hline H11-55-AP & FRONT & PADDED IMPACT, LOWER CENTRAL FACEMASK & H-III, M50 \\
\hline H11-55-B & OBLIQUE & PADDED IMPACT, UPPER OBLIQUE FACEMASK & H-III, M50 \\
\hline H11-55-C & SIDE & PADDED IMPACT, SIDE OF SHELL, OBLIQUE & H-III, M50 \\
\hline H11-55-D & OBLIQUE & PADDED IMPACT, REAR BOSS OF SHELL & H-III, M50 \\
\hline H11-55-F & FRONT & PADDED IMPACT, FRONT OF SHELL & H-III, M50 \\
\hline H11-55-R & REAR & PADDED IMPACT, REAR OF SHELL & H-III, M50 \\
\hline H11-55-UT & OBLIQUE & PADDED IMPACT, SIDE OF FACEMASK & H-III, M50 \\
\hline H11-74-A & OBLIQUE & PADDED IMPACT, OBLIQUE FACEMASK & H-III, M50 \\
\hline H11-74-AP & FRONT & PADDED IMPACT, LOWER CENTRAL FACEMASK & H-III, M50 \\
\hline H11-74-B & OBLIQUE & PADDED IMPACT, UPPER OBLIQUE FACEMASK & H-III, M50 \\
\hline H11-74-C & SIDE & PADDED IMPACT, SIDE OF SHELL, OBLIQUE & H-III, M50 \\
\hline H11-74-D & OBLIQUE & PADDED IMPACT, REAR BOSS OF SHELL & H-III, M50 \\
\hline H11-74-F & FRONT & PADDED IMPACT, FRONT OF SHELL & H-III, M50 \\
\hline H11-74-R & REAR & PADDED IMPACT, REAR OF SHELL & H-III, M50 \\
\hline H11-74-UT & OBLIQUE & PADDED IMPACT, SIDE OF FACEMASK & H-III, M50 \\
\hline H11-93-A & OBLIQUE & PADDED IMPACT, OBLIQUE FACEMASK & H-III, M50 \\
\hline H11-93-AP & FRONT & PADDED IMPACT, LOWER CENTRAL FACEMASK & H-III, M50 \\
\hline H11-93-B & OBLIQUE & PADDED IMPACT, UPPER OBLIQUE FACEMASK & H-III, M50 \\
\hline $\mathrm{H} 11-93-\mathrm{C}$ & SIDE & PADDED IMPACT, SIDE OF SHELL, OBLIQUE & H-III, M50 \\
\hline H11-93-D & OBLIQUE & PADDED IMPACT, REAR BOSS OF SHELL & H-III, M50 \\
\hline H11-93-F & FRONT & PADDED IMPACT, FRONT OF SHELL & H-III, M50 \\
\hline H11-93-R & REAR & PADDED IMPACT, REAR OF SHELL & H-III, M50 \\
\hline H11-93-UT & OBLIQUE & PADDED IMPACT, SIDE OF FACEMASK & H-III, M50 \\
\hline H12-55-A & OBLIQUE & PADDED IMPACT, OBLIQUE FACEMASK & H-III, M50 \\
\hline H12-55-AP & FRONT & PADDED IMPACT, LOWER CENTRAL FACEMASK & H-III, M50 \\
\hline H12-55-B & OBLIQUE & PADDED IMPACT, UPPER OBLIQUE FACEMASK & H-III, M50 \\
\hline $\mathrm{H} 12-55-\mathrm{C}$ & SIDE & PADDED IMPACT, SIDE OF SHELL, OBLIQUE & H-III, M50 \\
\hline H12-55-D & OBLIQUE & PADDED IMPACT, REAR BOSS OF SHELL & H-III, M50 \\
\hline $\mathrm{H} 12-55-\mathrm{F}$ & FRONT & PADDED IMPACT, FRONT OF SHELL & H-III, M50 \\
\hline H12-55-R & REAR & PADDED IMPACT, REAR OF SHELL & H-III, M50 \\
\hline H12-55-UT & OBLIQUE & PADDED IMPACT, SIDE OF FACEMASK & H-III, M50 \\
\hline H12-74-A & OBLIQUE & PADDED IMPACT, OBLIQUE FACEMASK & H-III, M50 \\
\hline H12-74-AP & FRONT & PADDED IMPACT, LOWER CENTRAL FACEMASK & H-III, M50 \\
\hline H12-74-B & OBLIQUE & PADDED IMPACT, UPPER OBLIQUE FACEMASK & H-III, M50 \\
\hline $\mathrm{H} 12-74-\mathrm{C}$ & SIDE & PADDED IMPACT, SIDE OF SHELL, OBLIQUE & H-III, M50 \\
\hline H12-74-D & OBLIQUE & PADDED IMPACT, REAR BOSS OF SHELL & H-III, M50 \\
\hline H12-74-F & FRONT & PADDED IMPACT, FRONT OF SHELL & H-III, M50 \\
\hline H12-74-R & REAR & PADDED IMPACT, REAR OF SHELL & H-III, M50 \\
\hline H12-74-UT & OBLIQUE & PADDED IMPACT, SIDE OF FACEMASK & H-III, M50 \\
\hline H12-93-A & OBLIQUE & PADDED IMPACT, OBLIQUE FACEMASK & H-III, M50 \\
\hline H12-93-AP & FRONT & PADDED IMPACT, LOWER CENTRAL FACEMASK & H-III, M50 \\
\hline H12-93-B & OBLIQUE & PADDED IMPACT, UPPER OBLIQUE FACEMASK & H-III, M50 \\
\hline $\mathrm{H} 12-93-\mathrm{C}$ & SIDE & PADDED IMPACT, SIDE OF SHELL, OBLIQUE & H-III, M50 \\
\hline H12-93-D & OBLIQUE & PADDED IMPACT, REAR BOSS OF SHELL & H-III, M50 \\
\hline H12-93-F & FRONT & PADDED IMPACT, FRONT OF SHELL & H-III, M50 \\
\hline H12-93-R & REAR & PADDED IMPACT, REAR OF SHELL & H-III, M50 \\
\hline H12-93-UT & OBLIQUE & PADDED IMPACT, SIDE OF FACEMASK & H-III, M50 \\
\hline H13-55-A & OBLIQUE & PADDED IMPACT, OBLIQUE FACEMASK & H-III, M50 \\
\hline H13-55-AP & FRONT & PADDED IMPACT, LOWER CENTRAL FACEMASK & H-III, M50 \\
\hline H13-55-B & OBLIQUE & PADDED IMPACT, UPPER OBLIQUE FACEMASK & H-III, M50 \\
\hline
\end{tabular}




\begin{tabular}{|c|c|c|c|}
\hline $\mathrm{H} 13-55-\mathrm{C}$ & SIDE & PADDED IMPACT, SIDE OF SHELL, OBLIQUE & H-III, M50 \\
\hline H13-55-D & OBLIQUE & PADDED IMPACT, REAR BOSS OF SHELL & H-III, M50 \\
\hline $\mathrm{H} 13-55-\mathrm{F}$ & FRONT & PADDED IMPACT, FRONT OF SHELL & H-III, M50 \\
\hline H13-55-R & REAR & PADDED IMPACT, REAR OF SHELL & H-III, M50 \\
\hline H13-55-UT & OBLIQUE & PADDED IMPACT, SIDE OF FACEMASK & H-III, M50 \\
\hline H13-74-A & OBLIQUE & PADDED IMPACT, OBLIQUE FACEMASK & H-III, M50 \\
\hline H13-74-AP & FRONT & PADDED IMPACT, LOWER CENTRAL FACEMASK & H-III, M50 \\
\hline H13-74-B & OBLIQUE & PADDED IMPACT, UPPER OBLIQUE FACEMASK & H-III, M50 \\
\hline H13-74-C & SIDE & PADDED IMPACT, SIDE OF SHELL, OBLIQUE & H-III, M50 \\
\hline H13-74-D & OBLIQUE & PADDED IMPACT, REAR BOSS OF SHELL & H-III, M50 \\
\hline H13-74-F & FRONT & PADDED IMPACT, FRONT OF SHELL & H-III, M50 \\
\hline H13-74-R & REAR & PADDED IMPACT, REAR OF SHELL & H-III, M50 \\
\hline H13-74-UT & OBLIQUE & PADDED IMPACT, SIDE OF FACEMASK & H-III, M50 \\
\hline H13-93-A & OBLIQUE & PADDED IMPACT, OBLIQUE FACEMASK & H-III, M50 \\
\hline H13-93-AP & FRONT & PADDED IMPACT, LOWER CENTRAL FACEMASK & H-III, M50 \\
\hline H13-93-B & OBLIQUE & PADDED IMPACT, UPPER OBLIQUE FACEMASK & H-III, M50 \\
\hline H13-93-C & SIDE & PADDED IMPACT, SIDE OF SHELL, OBLIQUE & H-III, M50 \\
\hline H13-93-D & OBLIQUE & PADDED IMPACT, REAR BOSS OF SHELL & H-III, M50 \\
\hline H13-93-F & FRONT & PADDED IMPACT, FRONT OF SHELL & H-III, M50 \\
\hline H13-93-R & REAR & PADDED IMPACT, REAR OF SHELL & H-III, M50 \\
\hline H13-93-UT & OBLIQUE & PADDED IMPACT, SIDE OF FACEMASK & H-III, M50 \\
\hline H14-55-A & OBLIQUE & PADDED IMPACT, OBLIQUE FACEMASK & H-III, M50 \\
\hline H14-55-AP & FRONT & PADDED IMPACT, LOWER CENTRAL FACEMASK & H-III, M50 \\
\hline H14-55-B & OBLIQUE & PADDED IMPACT, UPPER OBLIQUE FACEMASK & H-III, M50 \\
\hline $\mathrm{H} 14-55-\mathrm{C}$ & SIDE & PADDED IMPACT, SIDE OF SHELL, OBLIQUE & H-III, M50 \\
\hline H14-55-D & OBLIQUE & PADDED IMPACT, REAR BOSS OF SHELL & H-III, M50 \\
\hline $\mathrm{H} 14-55-\mathrm{F}$ & FRONT & PADDED IMPACT, FRONT OF SHELL & H-III, M50 \\
\hline H14-55-R & REAR & PADDED IMPACT, REAR OF SHELL & H-III, M50 \\
\hline H14-55-UT & OBLIQUE & PADDED IMPACT, SIDE OF FACEMASK & H-III, M50 \\
\hline H14-74-A & OBLIQUE & PADDED IMPACT, OBLIQUE FACEMASK & H-III, M50 \\
\hline H14-74-AP & FRONT & PADDED IMPACT, LOWER CENTRAL FACEMASK & H-III, M50 \\
\hline H14-74-B & OBLIQUE & PADDED IMPACT, UPPER OBLIQUE FACEMASK & H-III, M50 \\
\hline $\mathrm{H} 14-74-\mathrm{C}$ & SIDE & PADDED IMPACT, SIDE OF SHELL, OBLIQUE & H-III, M50 \\
\hline H14-74-D & OBLIQUE & PADDED IMPACT, REAR BOSS OF SHELL & H-III, M50 \\
\hline H14-74-F & FRONT & PADDED IMPACT, FRONT OF SHELL & H-III, M50 \\
\hline H14-74-R & REAR & PADDED IMPACT, REAR OF SHELL & H-III, M50 \\
\hline H14-74-UT & OBLIQUE & PADDED IMPACT, SIDE OF FACEMASK & H-III, M50 \\
\hline H14-93-A & OBLIQUE & PADDED IMPACT, OBLIQUE FACEMASK & H-III, M50 \\
\hline H14-93-AP & FRONT & PADDED IMPACT, LOWER CENTRAL FACEMASK & H-III, M50 \\
\hline H14-93-B & OBLIQUE & PADDED IMPACT, UPPER OBLIQUE FACEMASK & H-III, M50 \\
\hline H14-93-C & SIDE & PADDED IMPACT, SIDE OF SHELL, OBLIQUE & H-III, M50 \\
\hline H14-93-D & OBLIQUE & PADDED IMPACT, REAR BOSS OF SHELL & H-III, M50 \\
\hline $\mathrm{H} 14-93-\mathrm{F}$ & FRONT & PADDED IMPACT, FRONT OF SHELL & H-III, M50 \\
\hline H14-93-R & REAR & PADDED IMPACT, REAR OF SHELL & H-III, M50 \\
\hline H14-93-UT & OBLIQUE & PADDED IMPACT, SIDE OF FACEMASK & H-III, M50 \\
\hline H15-55-A & OBLIQUE & PADDED IMPACT, OBLIQUE FACEMASK & H-III, M50 \\
\hline H15-55-AP & FRONT & PADDED IMPACT, LOWER CENTRAL FACEMASK & H-III, M50 \\
\hline H15-55-B & OBLIQUE & PADDED IMPACT, UPPER OBLIQUE FACEMASK & H-III, M50 \\
\hline $\mathrm{H} 15-55-\mathrm{C}$ & SIDE & PADDED IMPACT, SIDE OF SHELL, OBLIQUE & H-III, M50 \\
\hline H15-55-D & OBLIQUE & PADDED IMPACT, REAR BOSS OF SHELL & H-III, M50 \\
\hline $\mathrm{H} 15-55-\mathrm{F}$ & FRONT & PADDED IMPACT, FRONT OF SHELL & H-III, M50 \\
\hline H15-55-R & REAR & PADDED IMPACT, REAR OF SHELL & H-III, M50 \\
\hline H15-55-UT & OBLIQUE & PADDED IMPACT, SIDE OF FACEMASK & H-III, M50 \\
\hline H15-74-A & OBLIQUE & PADDED IMPACT, OBLIQUE FACEMASK & H-III, M50 \\
\hline H15-74-AP & FRONT & PADDED IMPACT, LOWER CENTRAL FACEMASK & H-III, M50 \\
\hline H15-74-B & OBLIQUE & PADDED IMPACT, UPPER OBLIQUE FACEMASK & H-III, M50 \\
\hline $\mathrm{H} 15-74-\mathrm{C}$ & SIDE & PADDED IMPACT, SIDE OF SHELL, OBLIQUE & H-III, M50 \\
\hline H15-74-D & OBLIQUE & PADDED IMPACT, REAR BOSS OF SHELL & H-III, M50 \\
\hline H15-74-F & FRONT & PADDED IMPACT, FRONT OF SHELL & H-III, M50 \\
\hline H15-74-R & REAR & PADDED IMPACT, REAR OF SHELL & H-III, M50 \\
\hline H15-74-UT & OBLIQUE & PADDED IMPACT, SIDE OF FACEMASK & H-III, M50 \\
\hline H15-93-A & OBLIQUE & PADDED IMPACT, OBLIQUE FACEMASK & H-III, M50 \\
\hline
\end{tabular}




\begin{tabular}{|c|c|c|c|}
\hline H15-93-AP & FRONT & PADDED IMPACT, LOWER CENTRAL FACEMASK & H-III, M50 \\
\hline H15-93-B & OBLIQUE & PADDED IMPACT, UPPER OBLIQUE FACEMASK & H-III, M50 \\
\hline $\mathrm{H} 15-93-\mathrm{C}$ & SIDE & PADDED IMPACT, SIDE OF SHELL, OBLIQUE & H-III, M50 \\
\hline H15-93-D & OBLIQUE & PADDED IMPACT, REAR BOSS OF SHELL & H-III, M50 \\
\hline H15-93-F & FRONT & PADDED IMPACT, FRONT OF SHELL & H-III, M50 \\
\hline H15-93-R & REAR & PADDED IMPACT, REAR OF SHELL & H-III, M50 \\
\hline H15-93-UT & OBLIQUE & PADDED IMPACT, SIDE OF FACEMASK & H-III, M50 \\
\hline H16-55-A & OBLIQUE & PADDED IMPACT, OBLIQUE FACEMASK & H-III, M50 \\
\hline H16-55-AP & FRONT & PADDED IMPACT, LOWER CENTRAL FACEMASK & H-III, M50 \\
\hline H16-55-B & OBLIQUE & PADDED IMPACT, UPPER OBLIQUE FACEMASK & H-III, M50 \\
\hline H16-55-C & SIDE & PADDED IMPACT, SIDE OF SHELL, OBLIQUE & H-III, M50 \\
\hline H16-55-D & OBLIQUE & PADDED IMPACT, REAR BOSS OF SHELL & H-III, M50 \\
\hline H16-55-F & FRONT & PADDED IMPACT, FRONT OF SHELL & H-III, M50 \\
\hline H16-55-R & REAR & PADDED IMPACT, REAR OF SHELL & H-III, M50 \\
\hline H16-55-UT & OBLIQUE & PADDED IMPACT, SIDE OF FACEMASK & H-III, M50 \\
\hline H16-74-A & OBLIQUE & PADDED IMPACT, OBLIQUE FACEMASK & H-III, M50 \\
\hline H16-74-AP & FRONT & PADDED IMPACT, LOWER CENTRAL FACEMASK & H-III, M50 \\
\hline H16-74-B & OBLIQUE & PADDED IMPACT, UPPER OBLIQUE FACEMASK & H-III, M50 \\
\hline H16-74-C & SIDE & PADDED IMPACT, SIDE OF SHELL, OBLIQUE & H-III, M50 \\
\hline H16-74-D & OBLIQUE & PADDED IMPACT, REAR BOSS OF SHELL & H-III, M50 \\
\hline H16-74-F & FRONT & PADDED IMPACT, FRONT OF SHELL & H-III, M50 \\
\hline H16-74-R & REAR & PADDED IMPACT, REAR OF SHELL & H-III, M50 \\
\hline H16-74-UT & OBLIQUE & PADDED IMPACT, SIDE OF FACEMASK & H-III, M50 \\
\hline H16-93-A & OBLIQUE & PADDED IMPACT, OBLIQUE FACEMASK & H-III, M50 \\
\hline H16-93-AP & FRONT & PADDED IMPACT, LOWER CENTRAL FACEMASK & H-III, M50 \\
\hline H16-93-B & OBLIQUE & PADDED IMPACT, UPPER OBLIQUE FACEMASK & H-III, M50 \\
\hline H16-93-C & SIDE & PADDED IMPACT, SIDE OF SHELL, OBLIQUE & H-III, M50 \\
\hline H16-93-D & OBLIQUE & PADDED IMPACT, REAR BOSS OF SHELL & H-III, M50 \\
\hline H16-93-F & FRONT & PADDED IMPACT, FRONT OF SHELL & H-III, M50 \\
\hline H16-93-R & REAR & PADDED IMPACT, REAR OF SHELL & H-III, M50 \\
\hline H16-93-UT & OBLIQUE & PADDED IMPACT, SIDE OF FACEMASK & H-III, M50 \\
\hline H17-55-A & OBLIQUE & PADDED IMPACT, OBLIQUE FACEMASK & H-III, M50 \\
\hline H17-55-AP & FRONT & PADDED IMPACT, LOWER CENTRAL FACEMASK & H-III, M50 \\
\hline H17-55-B & OBLIQUE & PADDED IMPACT, UPPER OBLIQUE FACEMASK & H-III, M50 \\
\hline $\mathrm{H} 17-55-\mathrm{C}$ & SIDE & PADDED IMPACT, SIDE OF SHELL, OBLIQUE & H-III, M50 \\
\hline H17-55-D & OBLIQUE & PADDED IMPACT, REAR BOSS OF SHELL & H-III, M50 \\
\hline H17-55-F & FRONT & PADDED IMPACT, FRONT OF SHELL & H-III, M50 \\
\hline H17-55-R & REAR & PADDED IMPACT, REAR OF SHELL & H-III, M50 \\
\hline H17-55-UT & OBLIQUE & PADDED IMPACT, SIDE OF FACEMASK & H-III, M50 \\
\hline H17-74-A & OBLIQUE & PADDED IMPACT, OBLIQUE FACEMASK & H-III, M50 \\
\hline H17-74-AP & FRONT & PADDED IMPACT, LOWER CENTRAL FACEMASK & H-III, M50 \\
\hline H17-74-B & OBLIQUE & PADDED IMPACT, UPPER OBLIQUE FACEMASK & H-III, M50 \\
\hline $\mathrm{H} 17-74-\mathrm{C}$ & SIDE & PADDED IMPACT, SIDE OF SHELL, OBLIQUE & H-III, M50 \\
\hline H17-74-D & OBLIQUE & PADDED IMPACT, REAR BOSS OF SHELL & H-III, M50 \\
\hline H17-74-F & FRONT & PADDED IMPACT, FRONT OF SHELL & H-III, M50 \\
\hline H17-74-R & REAR & PADDED IMPACT, REAR OF SHELL & H-III, M50 \\
\hline H17-74-UT & OBLIQUE & PADDED IMPACT, SIDE OF FACEMASK & H-III, M50 \\
\hline H17-93-A & OBLIQUE & PADDED IMPACT, OBLIQUE FACEMASK & H-III, M50 \\
\hline H17-93-AP & FRONT & PADDED IMPACT, LOWER CENTRAL FACEMASK & H-III, M50 \\
\hline H17-93-B & OBLIQUE & PADDED IMPACT, UPPER OBLIQUE FACEMASK & H-III, M50 \\
\hline $\mathrm{H} 17-93-\mathrm{C}$ & SIDE & PADDED IMPACT, SIDE OF SHELL, OBLIQUE & H-III, M50 \\
\hline H17-93-D & OBLIQUE & PADDED IMPACT, REAR BOSS OF SHELL & H-III, M50 \\
\hline H17-93-F & FRONT & PADDED IMPACT, FRONT OF SHELL & H-III, M50 \\
\hline H17-93-R & REAR & PADDED IMPACT, REAR OF SHELL & H-III, M50 \\
\hline H17-93-UT & OBLIQUE & PADDED IMPACT, SIDE OF FACEMASK & H-III, M50 \\
\hline H18-55-A & OBLIQUE & PADDED IMPACT, OBLIQUE FACEMASK & H-III, M50 \\
\hline H18-55-AP & FRONT & PADDED IMPACT, LOWER CENTRAL FACEMASK & H-III, M50 \\
\hline H18-55-B & OBLIQUE & PADDED IMPACT, UPPER OBLIQUE FACEMASK & H-III, M50 \\
\hline $\mathrm{H} 18-55-\mathrm{C}$ & SIDE & PADDED IMPACT, SIDE OF SHELL, OBLIQUE & H-III, M50 \\
\hline H18-55-D & OBLIQUE & PADDED IMPACT, REAR BOSS OF SHELL & H-III, M50 \\
\hline H18-55-F & FRONT & PADDED IMPACT, FRONT OF SHELL & H-III, M50 \\
\hline H18-55-R & REAR & PADDED IMPACT, REAR OF SHELL & H-III, M50 \\
\hline
\end{tabular}




\begin{tabular}{|c|c|c|c|}
\hline H18-55-UT & OBLIQUE & PADDED IMPACT, SIDE OF FACEMASK & H-III, M50 \\
\hline H18-74-A & OBLIQUE & PADDED IMPACT, OBLIQUE FACEMASK & H-III, M50 \\
\hline H18-74-AP & FRONT & PADDED IMPACT, LOWER CENTRAL FACEMASK & H-III, M50 \\
\hline H18-74-B & OBLIQUE & PADDED IMPACT, UPPER OBLIQUE FACEMASK & H-III, M50 \\
\hline H18-74-C & SIDE & PADDED IMPACT, SIDE OF SHELL, OBLIQUE & H-III, M50 \\
\hline H18-74-D & OBLIQUE & PADDED IMPACT, REAR BOSS OF SHELL & H-III, M50 \\
\hline H18-74-F & FRONT & PADDED IMPACT, FRONT OF SHELL & H-III, M50 \\
\hline H18-74-R & REAR & PADDED IMPACT, REAR OF SHELL & H-III, M50 \\
\hline H18-74-UT & OBLIQUE & PADDED IMPACT, SIDE OF FACEMASK & H-III, M50 \\
\hline H18-93-A & OBLIQUE & PADDED IMPACT, OBLIQUE FACEMASK & H-III, M50 \\
\hline H18-93-AP & FRONT & PADDED IMPACT, LOWER CENTRAL FACEMASK & H-III, M50 \\
\hline H18-93-B & OBLIQUE & PADDED IMPACT, UPPER OBLIQUE FACEMASK & H-III, M50 \\
\hline $\mathrm{H} 18-93-\mathrm{C}$ & SIDE & PADDED IMPACT, SIDE OF SHELL, OBLIQUE & H-III, M50 \\
\hline H18-93-D & OBLIQUE & PADDED IMPACT, REAR BOSS OF SHELL & H-III, M50 \\
\hline H18-93-F & FRONT & PADDED IMPACT, FRONT OF SHELL & H-III, M50 \\
\hline H18-93-R & REAR & PADDED IMPACT, REAR OF SHELL & H-III, M50 \\
\hline H18-93-UT & OBLIQUE & PADDED IMPACT, SIDE OF FACEMASK & H-III, M50 \\
\hline H19-55-A & OBLIQUE & PADDED IMPACT, OBLIQUE FACEMASK & H-III, M50 \\
\hline H19-55-AP & FRONT & PADDED IMPACT, LOWER CENTRAL FACEMASK & H-III, M50 \\
\hline H19-55-B & OBLIQUE & PADDED IMPACT, UPPER OBLIQUE FACEMASK & H-III, M50 \\
\hline $\mathrm{H} 19-55-\mathrm{C}$ & SIDE & PADDED IMPACT, SIDE OF SHELL, OBLIQUE & H-III, M50 \\
\hline H19-55-D & OBLIQUE & PADDED IMPACT, REAR BOSS OF SHELL & H-III, M50 \\
\hline H19-55-F & FRONT & PADDED IMPACT, FRONT OF SHELL & H-III, M50 \\
\hline H19-55-R & REAR & PADDED IMPACT, REAR OF SHELL & H-III, M50 \\
\hline H19-55-UT & OBLIQUE & PADDED IMPACT, SIDE OF FACEMASK & H-III, M50 \\
\hline H19-74-A & OBLIQUE & PADDED IMPACT, OBLIQUE FACEMASK & H-III, M50 \\
\hline H19-74-AP & FRONT & PADDED IMPACT, LOWER CENTRAL FACEMASK & H-III, M50 \\
\hline H19-74-B & OBLIQUE & PADDED IMPACT, UPPER OBLIQUE FACEMASK & H-III, M50 \\
\hline H19-74-C & SIDE & PADDED IMPACT, SIDE OF SHELL, OBLIQUE & H-III, M50 \\
\hline H19-74-D & OBLIQUE & PADDED IMPACT, REAR BOSS OF SHELL & H-III, M50 \\
\hline H19-74-F & FRONT & PADDED IMPACT, FRONT OF SHELL & H-III, M50 \\
\hline H19-74-R & REAR & PADDED IMPACT, REAR OF SHELL & H-III, M50 \\
\hline H19-74-UT & OBLIQUE & PADDED IMPACT, SIDE OF FACEMASK & H-III, M50 \\
\hline H19-93-A & OBLIQUE & PADDED IMPACT, OBLIQUE FACEMASK & H-III, M50 \\
\hline H19-93-AP & FRONT & PADDED IMPACT, LOWER CENTRAL FACEMASK & H-III, M50 \\
\hline H19-93-B & OBLIQUE & PADDED IMPACT, UPPER OBLIQUE FACEMASK & H-III, M50 \\
\hline H19-93-C & SIDE & PADDED IMPACT, SIDE OF SHELL, OBLIQUE & H-III, M50 \\
\hline H19-93-D & OBLIQUE & PADDED IMPACT, REAR BOSS OF SHELL & H-III, M50 \\
\hline H19-93-F & FRONT & PADDED IMPACT, FRONT OF SHELL & H-III, M50 \\
\hline H19-93-R & REAR & PADDED IMPACT, REAR OF SHELL & H-III, M50 \\
\hline H19-93-UT & OBLIQUE & PADDED IMPACT, SIDE OF FACEMASK & H-III, M50 \\
\hline H20-55-A-R1 & OBLIQUE & PADDED IMPACT, OBLIQUE FACEMASK & H-III, M50 \\
\hline H20-55-AP-R1 & FRONT & PADDED IMPACT, LOWER CENTRAL FACEMASK & H-III, M50 \\
\hline H20-55-B-R1 & OBLIQUE & PADDED IMPACT, UPPER OBLIQUE FACEMASK & H-III, M50 \\
\hline $\mathrm{H} 20-55-\mathrm{C}-\mathrm{R} 1$ & SIDE & PADDED IMPACT, SIDE OF SHELL, OBLIQUE & H-III, M50 \\
\hline H20-55-D-R1 & OBLIQUE & PADDED IMPACT, REAR BOSS OF SHELL & H-III, M50 \\
\hline H20-55-F-R1 & FRONT & PADDED IMPACT, FRONT OF SHELL & H-III, M50 \\
\hline H20-55-R-R1 & REAR & PADDED IMPACT, REAR OF SHELL & H-III, M50 \\
\hline H20-55-UT-R1 & OBLIQUE & PADDED IMPACT, SIDE OF FACEMASK & H-III, M50 \\
\hline H20-74-A-R1 & OBLIQUE & PADDED IMPACT, OBLIQUE FACEMASK & H-III, M50 \\
\hline H20-74-AP-R1 & FRONT & PADDED IMPACT, LOWER CENTRAL FACEMASK & H-III, M50 \\
\hline $\mathrm{H} 20-74-\mathrm{B}-\mathrm{R} 1$ & OBLIQUE & PADDED IMPACT, UPPER OBLIQUE FACEMASK & H-III, M50 \\
\hline H20-74-C-R1 & SIDE & PADDED IMPACT, SIDE OF SHELL, OBLIQUE & H-III, M50 \\
\hline H20-74-D-R1 & OBLIQUE & PADDED IMPACT, REAR BOSS OF SHELL & H-III, M50 \\
\hline H20-74-F-R1 & FRONT & PADDED IMPACT, FRONT OF SHELL & H-III, M50 \\
\hline H20-74-R-R1 & REAR & PADDED IMPACT, REAR OF SHELL & H-III, M50 \\
\hline H20-74-UT-R1 & OBLIQUE & PADDED IMPACT, SIDE OF FACEMASK & H-III, M50 \\
\hline H20-93-A-R1 & OBLIQUE & PADDED IMPACT, OBLIQUE FACEMASK & H-III, M50 \\
\hline H20-93-AP-R1 & FRONT & PADDED IMPACT, LOWER CENTRAL FACEMASK & H-III, M50 \\
\hline H20-93-B-R1 & OBLIQUE & PADDED IMPACT, UPPER OBLIQUE FACEMASK & H-III, M50 \\
\hline H20-93-C-R1 & SIDE & PADDED IMPACT, SIDE OF SHELL, OBLIQUE & H-III, M50 \\
\hline H20-93-D-R1 & OBLIQUE & PADDED IMPACT, REAR BOSS OF SHELL & H-III, M50 \\
\hline
\end{tabular}




\begin{tabular}{|c|c|c|c|}
\hline H20-93-F-R1 & FRONT & PADDED IMPACT, FRONT OF SHELL & H-III, M50 \\
\hline H20-93-R-R1 & REAR & PADDED IMPACT, REAR OF SHELL & H-III, M50 \\
\hline H20-93-UT-R1 & OBLIQUE & PADDED IMPACT, SIDE OF FACEMASK & H-III, M50 \\
\hline H21-55-A-R1 & OBLIQUE & PADDED IMPACT, OBLIQUE FACEMASK & H-III, M50 \\
\hline H21-55-AP-R1 & FRONT & PADDED IMPACT, LOWER CENTRAL FACEMASK & H-III, M50 \\
\hline H21-55-B-R1 & OBLIQUE & PADDED IMPACT, UPPER OBLIQUE FACEMASK & H-III, M50 \\
\hline H21-55-C-R1 & SIDE & PADDED IMPACT, SIDE OF SHELL, OBLIQUE & H-III, M50 \\
\hline H21-55-D-R1 & OBLIQUE & PADDED IMPACT, REAR BOSS OF SHELL & H-III, M50 \\
\hline H21-55-F-R1 & FRONT & PADDED IMPACT, FRONT OF SHELL & H-III, M50 \\
\hline H21-55-R-R1 & REAR & PADDED IMPACT, REAR OF SHELL & H-III, M50 \\
\hline H21-55-UT-R1 & OBLIQUE & PADDED IMPACT, SIDE OF FACEMASK & H-III, M50 \\
\hline H21-74-A-R1 & OBLIQUE & PADDED IMPACT, OBLIQUE FACEMASK & H-III, M50 \\
\hline H21-74-AP-R1 & FRONT & PADDED IMPACT, LOWER CENTRAL FACEMASK & H-III, M50 \\
\hline H21-74-B-R1 & OBLIQUE & PADDED IMPACT, UPPER OBLIQUE FACEMASK & H-III, M50 \\
\hline H21-74-C-R1 & SIDE & PADDED IMPACT, SIDE OF SHELL, OBLIQUE & H-III, M50 \\
\hline H21-74-D-R1 & OBLIQUE & PADDED IMPACT, REAR BOSS OF SHELL & H-III, M50 \\
\hline H21-74-F-R1 & FRONT & PADDED IMPACT, FRONT OF SHELL & H-III, M50 \\
\hline H21-74-R-R1 & REAR & PADDED IMPACT, REAR OF SHELL & H-III, M50 \\
\hline H21-74-UT-R1 & OBLIQUE & PADDED IMPACT, SIDE OF FACEMASK & H-III, M50 \\
\hline H21-93-A-R1 & OBLIQUE & PADDED IMPACT, OBLIQUE FACEMASK & H-III, M50 \\
\hline H21-93-AP-R1 & FRONT & PADDED IMPACT, LOWER CENTRAL FACEMASK & H-III, M50 \\
\hline H21-93-B-R1 & OBLIQUE & PADDED IMPACT, UPPER OBLIQUE FACEMASK & H-III, M50 \\
\hline H21-93-C-R1 & SIDE & PADDED IMPACT, SIDE OF SHELL, OBLIQUE & H-III, M50 \\
\hline H21-93-D-R1 & OBLIQUE & PADDED IMPACT, REAR BOSS OF SHELL & H-III, M50 \\
\hline H21-93-F-R1 & FRONT & PADDED IMPACT, FRONT OF SHELL & H-III, M50 \\
\hline H21-93-R-R1 & REAR & PADDED IMPACT, REAR OF SHELL & H-III, M50 \\
\hline H21-93-UT-R1 & OBLIQUE & PADDED IMPACT, SIDE OF FACEMASK & H-III, M50 \\
\hline H22-55-A-R1 & OBLIQUE & PADDED IMPACT, OBLIQUE FACEMASK & H-III, M50 \\
\hline H22-55-AP-R1 & FRONT & PADDED IMPACT, LOWER CENTRAL FACEMASK & H-III, M50 \\
\hline H22-55-B-R1 & OBLIQUE & PADDED IMPACT, UPPER OBLIQUE FACEMASK & H-III, M50 \\
\hline $\mathrm{H} 22-55-\mathrm{C}-\mathrm{R} 1$ & SIDE & PADDED IMPACT, SIDE OF SHELL, OBLIQUE & H-III, M50 \\
\hline H22-55-D-R1 & OBLIQUE & PADDED IMPACT, REAR BOSS OF SHELL & H-III, M50 \\
\hline H22-55-F-R1 & FRONT & PADDED IMPACT, FRONT OF SHELL & H-III, M50 \\
\hline H22-55-R-R1 & REAR & PADDED IMPACT, REAR OF SHELL & H-III, M50 \\
\hline H22-55-UT-R1 & OBLIQUE & PADDED IMPACT, SIDE OF FACEMASK & H-III, M50 \\
\hline H22-74-A-R1 & OBLIQUE & PADDED IMPACT, OBLIQUE FACEMASK & H-III, M50 \\
\hline H22-74-AP-R1 & FRONT & PADDED IMPACT, LOWER CENTRAL FACEMASK & H-III, M50 \\
\hline H22-74-B-R1 & OBLIQUE & PADDED IMPACT, UPPER OBLIQUE FACEMASK & H-III, M50 \\
\hline H22-74-C-R1 & SIDE & PADDED IMPACT, SIDE OF SHELL, OBLIQUE & H-III, M50 \\
\hline H22-74-D-R1 & OBLIQUE & PADDED IMPACT, REAR BOSS OF SHELL & H-III, M50 \\
\hline H22-74-F-R1 & FRONT & PADDED IMPACT, FRONT OF SHELL & H-III, M50 \\
\hline H22-74-R-R1 & REAR & PADDED IMPACT, REAR OF SHELL & H-III, M50 \\
\hline H22-74-UT-R1 & OBLIQUE & PADDED IMPACT, SIDE OF FACEMASK & H-III, M50 \\
\hline H22-93-A-R1 & OBLIQUE & PADDED IMPACT, OBLIQUE FACEMASK & H-III, M50 \\
\hline H22-93-AP-R1 & FRONT & PADDED IMPACT, LOWER CENTRAL FACEMASK & H-III, M50 \\
\hline H22-93-B-R1 & OBLIQUE & PADDED IMPACT, UPPER OBLIQUE FACEMASK & H-III, M50 \\
\hline H22-93-C-R1 & SIDE & PADDED IMPACT, SIDE OF SHELL, OBLIQUE & H-III, M50 \\
\hline H22-93-D-R1 & OBLIQUE & PADDED IMPACT, REAR BOSS OF SHELL & H-III, M50 \\
\hline H22-93-F-R1 & FRONT & PADDED IMPACT, FRONT OF SHELL & H-III, M50 \\
\hline H22-93-R-R1 & REAR & PADDED IMPACT, REAR OF SHELL & H-III, M50 \\
\hline H22-93-UT-R1 & OBLIQUE & PADDED IMPACT, SIDE OF FACEMASK & H-III, M50 \\
\hline H23-55-A-R1 & OBLIQUE & PADDED IMPACT, OBLIQUE FACEMASK & H-III, M50 \\
\hline H23-55-AP-R1 & FRONT & PADDED IMPACT, LOWER CENTRAL FACEMASK & H-III, M50 \\
\hline H23-55-B-R1 & OBLIQUE & PADDED IMPACT, UPPER OBLIQUE FACEMASK & H-III, M50 \\
\hline $\mathrm{H} 23-55-\mathrm{C}-\mathrm{R} 1$ & SIDE & PADDED IMPACT, SIDE OF SHELL, OBLIQUE & H-III, M50 \\
\hline H23-55-D-R1 & OBLIQUE & PADDED IMPACT, REAR BOSS OF SHELL & H-III, M50 \\
\hline $\mathrm{H} 23-55-\mathrm{F}-\mathrm{R} 1$ & FRONT & PADDED IMPACT, FRONT OF SHELL & H-III, M50 \\
\hline H23-55-R-R1 & REAR & PADDED IMPACT, REAR OF SHELL & H-III, M50 \\
\hline H23-55-UT-R1 & OBLIQUE & PADDED IMPACT, SIDE OF FACEMASK & H-III, M50 \\
\hline H23-74-A-R1 & OBLIQUE & PADDED IMPACT, OBLIQUE FACEMASK & H-III, M50 \\
\hline H23-74-AP-R1 & FRONT & PADDED IMPACT, LOWER CENTRAL FACEMASK & H-III, M50 \\
\hline H23-74-B-R1 & OBLIQUE & PADDED IMPACT, UPPER OBLIQUE FACEMASK & H-III, M50 \\
\hline
\end{tabular}




\begin{tabular}{|c|c|c|c|}
\hline H23-74-C-R1 & SIDE & PADDED IMPACT, SIDE OF SHELL, OBLIQUE & H-III, M50 \\
\hline H23-74-D-R1 & OBLIQUE & PADDED IMPACT, REAR BOSS OF SHELL & H-III, M50 \\
\hline H23-74-F-R1 & FRONT & PADDED IMPACT, FRONT OF SHELL & H-III, M50 \\
\hline H23-74-R-R1 & REAR & PADDED IMPACT, REAR OF SHELL & H-III, M50 \\
\hline H23-74-UT-R1 & OBLIQUE & PADDED IMPACT, SIDE OF FACEMASK & H-III, M50 \\
\hline H23-93-A-R1 & OBLIQUE & PADDED IMPACT, OBLIQUE FACEMASK & H-III, M50 \\
\hline H23-93-AP-R1 & FRONT & PADDED IMPACT, LOWER CENTRAL FACEMASK & H-III, M50 \\
\hline H23-93-B-R1 & OBLIQUE & PADDED IMPACT, UPPER OBLIQUE FACEMASK & H-III, M50 \\
\hline H23-93-C-R1 & SIDE & PADDED IMPACT, SIDE OF SHELL, OBLIQUE & H-III, M50 \\
\hline H23-93-D-R1 & OBLIQUE & PADDED IMPACT, REAR BOSS OF SHELL & H-III, M50 \\
\hline H23-93-F-R1 & FRONT & PADDED IMPACT, FRONT OF SHELL & H-III, M50 \\
\hline H23-93-R-R1 & REAR & PADDED IMPACT, REAR OF SHELL & H-III, M50 \\
\hline H23-93-UT-R1 & OBLIQUE & PADDED IMPACT, SIDE OF FACEMASK & H-III, M50 \\
\hline H24-55-A-R1 & OBLIQUE & PADDED IMPACT, OBLIQUE FACEMASK & H-III, M50 \\
\hline H24-55-AP-R1 & FRONT & PADDED IMPACT, LOWER CENTRAL FACEMASK & H-III, M50 \\
\hline H24-55-B-R1 & OBLIQUE & PADDED IMPACT, UPPER OBLIQUE FACEMASK & H-III, M50 \\
\hline H24-55-C-R1 & SIDE & PADDED IMPACT, SIDE OF SHELL, OBLIQUE & H-III, M50 \\
\hline H24-55-D-R1 & OBLIQUE & PADDED IMPACT, REAR BOSS OF SHELL & H-III, M50 \\
\hline H24-55-F-R1 & FRONT & PADDED IMPACT, FRONT OF SHELL & H-III, M50 \\
\hline H24-55-R-R1 & REAR & PADDED IMPACT, REAR OF SHELL & H-III, M50 \\
\hline H24-55-UT-R1 & OBLIQUE & PADDED IMPACT, SIDE OF FACEMASK & H-III, M50 \\
\hline H24-74-A-R1 & OBLIQUE & PADDED IMPACT, OBLIQUE FACEMASK & H-III, M50 \\
\hline H24-74-AP-R1 & FRONT & PADDED IMPACT, LOWER CENTRAL FACEMASK & H-III, M50 \\
\hline H24-74-B-R1 & OBLIQUE & PADDED IMPACT, UPPER OBLIQUE FACEMASK & H-III, M50 \\
\hline H24-74-C-R1 & SIDE & PADDED IMPACT, SIDE OF SHELL, OBLIQUE & H-III, M50 \\
\hline H24-74-D-R1 & OBLIQUE & PADDED IMPACT, REAR BOSS OF SHELL & H-III, M50 \\
\hline H24-74-F-R1 & FRONT & PADDED IMPACT, FRONT OF SHELL & H-III, M50 \\
\hline H24-74-R-R1 & REAR & PADDED IMPACT, REAR OF SHELL & H-III, M50 \\
\hline H24-74-UT-R1 & OBLIQUE & PADDED IMPACT, SIDE OF FACEMASK & H-III, M50 \\
\hline H24-93-A-R1 & OBLIQUE & PADDED IMPACT, OBLIQUE FACEMASK & H-III, M50 \\
\hline H24-93-AP-R1 & FRONT & PADDED IMPACT, LOWER CENTRAL FACEMASK & H-III, M50 \\
\hline H24-93-B-R1 & OBLIQUE & PADDED IMPACT, UPPER OBLIQUE FACEMASK & H-III, M50 \\
\hline H24-93-C-R1 & SIDE & PADDED IMPACT, SIDE OF SHELL, OBLIQUE & H-III, M50 \\
\hline H24-93-D-R1 & OBLIQUE & PADDED IMPACT, REAR BOSS OF SHELL & H-III, M50 \\
\hline H24-93-F-R1 & FRONT & PADDED IMPACT, FRONT OF SHELL & H-III, M50 \\
\hline H24-93-R-R1 & REAR & PADDED IMPACT, REAR OF SHELL & H-III, M50 \\
\hline H24-93-UT-R1 & OBLIQUE & PADDED IMPACT, SIDE OF FACEMASK & H-III, M50 \\
\hline
\end{tabular}

\section{J.6 NBDL Human Volunteer Sled Tests}

\begin{tabular}{clcc}
\hline Source ID & $\begin{array}{c}\text { Impact } \\
\text { Condition }\end{array}$ & Test Details & Surrogate \\
\hline LX3779 & FRONTAL & $0^{\circ}$ REARWARD (-GX) & HUMAN VOLUNTEER, 120, M \\
LX3780 & FRONTAL & $0^{\circ}$ REARWARD (-GX) & HUMAN VOLUNTEER, 127, M \\
LX3782 & FRONTAL & $0^{\circ}$ REARWARD (-GX) & HUMAN VOLUNTEER, 118, M \\
LX3783 & FRONTAL & $0^{\circ}$ REARWARD (-GX) & HUMAN VOLUNTEER, 131, M \\
LX3785 & FRONTAL & $0^{\circ}$ REARWARD (-GX) & HUMAN VOLUNTEER, 119, M \\
LX3786 & FRONTAL & $0^{\circ}$ REARWARD (-GX) & HUMAN VOLUNTEER, 134, M \\
LX3788 & FRONTAL & $0^{\circ}$ REARWARD (-GX) & HUMAN VOLUNTEER, 132, M \\
LX3789 & FRONTAL & $0^{\circ}$ REARWARD (-GX) & HUMAN VOLUNTEER, 130, M \\
LX3791 & FRONTAL & $0^{\circ}$ REARWARD (-GX) & HUMAN VOLUNTEER, 133, M \\
LX3793 & FRONTAL & $0^{\circ}$ REARWARD (-GX) & HUMAN VOLUNTEER, 120, M \\
LX3794 & FRONTAL & $0^{\circ}$ REARWARD (-GX) & HUMAN VOLUNTEER, 127, M \\
LX3796 & FRONTAL & $0^{\circ}$ REARWARD (-GX) & HUMAN VOLUNTEER, 118, M \\
LX3797 & FRONTAL & $0^{\circ}$ REARWARD (-GX) & HUMAN VOLUNTEER, 119, M \\
LX3798 & FRONTAL & $0^{\circ}$ REARWARD (-GX) & HUMAN VOLUNTEER, 133, M \\
LX3800 & FRONTAL & $0^{\circ}$ REARWARD (-GX) & HUMAN VOLUNTEER, 135, M \\
LX3801 & FRONTAL & $0^{\circ}$ REARWARD (-GX) & HUMAN VOLUNTEER, 136, M
\end{tabular}




\begin{tabular}{|c|c|c|c|}
\hline LX3803 & FRONTAL & $0^{\circ}$ REARWARD (-GX) & HUMAN VOLUNTEER, 130, M \\
\hline LX3804 & FRONTAL & $0^{\circ}$ REARWARD (-GX) & HUMAN VOLUNTEER, 131, M \\
\hline LX3805 & FRONTAL & $0^{\circ}$ REARWARD (-GX) & HUMAN VOLUNTEER, $132, \mathrm{M}$ \\
\hline LX3807 & FRONTAL & $0^{\circ}$ REARWARD (-GX) & HUMAN VOLUNTEER, 134, M \\
\hline LX3808 & FRONTAL & $0^{\circ}$ REARWARD (-GX) & HUMAN VOLUNTEER, 135, M \\
\hline LX3809 & FRONTAL & $0^{\circ}$ REARWARD (-GX) & HUMAN VOLUNTEER, 136, M \\
\hline LX3812 & FRONTAL & $0^{\circ}$ REARWARD (-GX) & HUMAN VOLUNTEER, 127, M \\
\hline LX3814 & FRONTAL & $0^{\circ}$ REARWARD (-GX) & HUMAN VOLUNTEER, 120, M \\
\hline LX3817 & FRONTAL & $0^{\circ}$ REARWARD (-GX) & HUMAN VOLUNTEER, 131, M \\
\hline LX3819 & FRONTAL & $0^{\circ}$ REARWARD (-GX) & HUMAN VOLUNTEER, 133, M \\
\hline LX3821 & FRONTAL & $0^{\circ}$ REARWARD (-GX) & HUMAN VOLUNTEER, 119, M \\
\hline LX3822 & FRONTAL & $0^{\circ}$ REARWARD (-GX) & HUMAN VOLUNTEER, 134, M \\
\hline LX3824 & FRONTAL & $0^{\circ}$ REARWARD (-GX) & HUMAN VOLUNTEER, $136, \mathrm{M}$ \\
\hline LX3833 & FRONTAL & $0^{\circ}$ REARWARD (-GX) & HUMAN VOLUNTEER, 118, M \\
\hline LX3837 & FRONTAL & $0^{\circ}$ REARWARD (-GX) & HUMAN VOLUNTEER, $118, \mathrm{M}$ \\
\hline LX3839 & FRONTAL & $0^{\circ}$ REARWARD (-GX) & HUMAN VOLUNTEER, 130, M \\
\hline LX3840 & FRONTAL & $0^{\circ}$ REARWARD (-GX) & HUMAN VOLUNTEER, 131, M \\
\hline LX3841 & FRONTAL & $0^{\circ}$ REARWARD (-GX) & HUMAN VOLUNTEER, 133, M \\
\hline LX3842 & FRONTAL & $0^{\circ}$ REARWARD (-GX) & HUMAN VOLUNTEER, 134, M \\
\hline LX3851 & FRONTAL & $0^{\circ}$ REARWARD (-GX) & HUMAN VOLUNTEER, 120, M \\
\hline LX3852 & FRONTAL & $0^{\circ}$ REARWARD (-GX) & HUMAN VOLUNTEER, 127, M \\
\hline LX3854 & FRONTAL & $0^{\circ}$ REARWARD (-GX) & HUMAN VOLUNTEER, $130, \mathrm{M}$ \\
\hline LX3856 & FRONTAL & $0^{\circ}$ REARWARD (-GX) & HUMAN VOLUNTEER, $118, \mathrm{M}$ \\
\hline LX3857 & FRONTAL & $0^{\circ}$ REARWARD (-GX) & HUMAN VOLUNTEER, 131, M \\
\hline LX3858 & FRONTAL & $0^{\circ}$ REARWARD (-GX) & HUMAN VOLUNTEER, 132, M \\
\hline LX3869 & FRONTAL & $0^{\circ}$ REARWARD (-GX) & HUMAN VOLUNTEER, 133, M \\
\hline LX3870 & FRONTAL & $0^{\circ}$ REARWARD (-GX) & HUMAN VOLUNTEER, $134, \mathrm{M}$ \\
\hline LX3871 & FRONTAL & $0^{\circ}$ REARWARD (-GX) & HUMAN VOLUNTEER, 135, M \\
\hline LX3872 & FRONTAL & $0^{\circ}$ REARWARD (-GX) & HUMAN VOLUNTEER, 136, M \\
\hline LX3875 & FRONTAL & $0^{\circ}$ REARWARD (-GX) & HUMAN VOLUNTEER, $118, \mathrm{M}$ \\
\hline LX3876 & FRONTAL & $0^{\circ}$ REARWARD (-GX) & HUMAN VOLUNTEER, 130, M \\
\hline LX3878 & FRONTAL & $0^{\circ}$ REARWARD (-GX) & HUMAN VOLUNTEER, $120, \mathrm{M}$ \\
\hline LX3880 & FRONTAL & $0^{\circ}$ REARWARD (-GX) & HUMAN VOLUNTEER, 118, M \\
\hline LX3882 & FRONTAL & $0^{\circ}$ REARWARD (-GX) & HUMAN VOLUNTEER, 120, M \\
\hline LX3883 & FRONTAL & $0^{\circ}$ REARWARD (-GX) & HUMAN VOLUNTEER, $127, \mathrm{M}$ \\
\hline LX3885 & FRONTAL & $0^{\circ}$ REARWARD (-GX) & HUMAN VOLUNTEER, 131, M \\
\hline LX3886 & FRONTAL & $0^{\circ}$ REARWARD (-GX) & HUMAN VOLUNTEER, $118, \mathrm{M}$ \\
\hline LX3887 & FRONTAL & $0^{\circ}$ REARWARD (-GX) & HUMAN VOLUNTEER, 132, M \\
\hline LX3889 & FRONTAL & $0^{\circ}$ REARWARD (-GX) & HUMAN VOLUNTEER, 130, M \\
\hline LX3890 & FRONTAL & $0^{\circ}$ REARWARD (-GX) & HUMAN VOLUNTEER, $134, \mathrm{M}$ \\
\hline LX3893 & FRONTAL & $0^{\circ}$ REARWARD (-GX) & HUMAN VOLUNTEER, 127, M \\
\hline LX3894 & FRONTAL & $0^{\circ}$ REARWARD (-GX) & HUMAN VOLUNTEER, $131, \mathrm{M}$ \\
\hline LX3895 & FRONTAL & $0^{\circ}$ REARWARD (-GX) & HUMAN VOLUNTEER, 133, M \\
\hline LX3898 & FRONTAL & $0^{\circ}$ REARWARD (-GX) & HUMAN VOLUNTEER, 135, M \\
\hline LX3900 & FRONTAL & $0^{\circ}$ REARWARD (-GX) & HUMAN VOLUNTEER, $132, \mathrm{M}$ \\
\hline LX3901 & FRONTAL & $0^{\circ}$ REARWARD (-GX) & HUMAN VOLUNTEER, 136, M \\
\hline LX3903 & FRONTAL & $0^{\circ}$ REARWARD (-GX) & HUMAN VOLUNTEER, $118, \mathrm{M}$ \\
\hline LX3904 & FRONTAL & $0^{\circ}$ REARWARD (-GX) & HUMAN VOLUNTEER, 127, M \\
\hline LX3906 & FRONTAL & $0^{\circ}$ REARWARD (-GX) & HUMAN VOLUNTEER, 120, M \\
\hline LX3908 & FRONTAL & $0^{\circ}$ REARWARD (-GX) & HUMAN VOLUNTEER, 131, M \\
\hline LX3909 & FRONTAL & $0^{\circ}$ REARWARD (-GX) & HUMAN VOLUNTEER, 132, M \\
\hline LX3913 & FRONTAL & $0^{\circ}$ REARWARD (-GX) & HUMAN VOLUNTEER, $133, \mathrm{M}$ \\
\hline LX3914 & FRONTAL & $0^{\circ}$ REARWARD (-GX) & HUMAN VOLUNTEER, 134, M \\
\hline LX3916 & FRONTAL & $0^{\circ}$ REARWARD (-GX) & HUMAN VOLUNTEER, 135, M \\
\hline LX3918 & FRONTAL & $0^{\circ}$ REARWARD (-GX) & HUMAN VOLUNTEER, 136, M \\
\hline LX3920 & FRONTAL & $0^{\circ}$ REARWARD (-GX) & HUMAN VOLUNTEER, 118, M \\
\hline LX3921 & FRONTAL & $0^{\circ}$ REARWARD (-GX) & HUMAN VOLUNTEER, $120, \mathrm{M}$ \\
\hline LX3924 & FRONTAL & $0^{\circ}$ REARWARD (-GX) & HUMAN VOLUNTEER, 127, M \\
\hline LX3926 & FRONTAL & $0^{\circ}$ REARWARD (-GX) & HUMAN VOLUNTEER, 131, M \\
\hline LX3927 & FRONTAL & $0^{\circ}$ REARWARD (-GX) & HUMAN VOLUNTEER, 132, M \\
\hline LX3928 & FRONTAL & $0^{\circ}$ REARWARD (-GX) & HUMAN VOLUNTEER, 130, M \\
\hline LX3939 & FRONTAL & $0^{\circ}$ REARWARD (-GX) & HUMAN VOLUNTEER, 133, M \\
\hline
\end{tabular}




\begin{tabular}{|c|c|c|c|}
\hline LX3940 & FRONTAL & $0^{\circ}$ REARWARD (-GX) & HUMAN VOLUNTEER, 134, M \\
\hline LX3941 & FRONTAL & $0^{\circ}$ REARWARD (-GX) & HUMAN VOLUNTEER, 135, M \\
\hline LX3942 & FRONTAL & $0^{\circ}$ REARWARD (-GX) & HUMAN VOLUNTEER, 136, M \\
\hline LX3944 & FRONTAL & $0^{\circ}$ REARWARD (-GX) & HUMAN VOLUNTEER, 130, M \\
\hline LX3945 & FRONTAL & $0^{\circ}$ REARWARD (-GX) & HUMAN VOLUNTEER, $118, \mathrm{M}$ \\
\hline LX3946 & FRONTAL & $0^{\circ}$ REARWARD (-GX) & HUMAN VOLUNTEER, $120, \mathrm{M}$ \\
\hline LX3948 & FRONTAL & $0^{\circ}$ REARWARD (-GX) & HUMAN VOLUNTEER, 131, M \\
\hline LX3949 & FRONTAL & $0^{\circ}$ REARWARD (-GX) & HUMAN VOLUNTEER, 127, M \\
\hline LX3950 & FRONTAL & $0^{\circ}$ REARWARD (-GX) & HUMAN VOLUNTEER, $132, \mathrm{M}$ \\
\hline LX3951 & FRONTAL & $0^{\circ}$ REARWARD (-GX) & HUMAN VOLUNTEER, 133, M \\
\hline LX3953 & FRONTAL & $0^{\circ}$ REARWARD (-GX) & HUMAN VOLUNTEER, $136, \mathrm{M}$ \\
\hline LX3954 & FRONTAL & $0^{\circ}$ REARWARD (-GX) & HUMAN VOLUNTEER, 120, M \\
\hline LX3955 & FRONTAL & $0^{\circ}$ REARWARD (-GX) & HUMAN VOLUNTEER, $135, \mathrm{M}$ \\
\hline LX3957 & FRONTAL & $0^{\circ}$ REARWARD (-GX) & HUMAN VOLUNTEER, 132, M \\
\hline LX3958 & FRONTAL & $0^{\circ}$ REARWARD (-GX) & HUMAN VOLUNTEER, $118, \mathrm{M}$ \\
\hline LX3959 & FRONTAL & $0^{\circ}$ REARWARD (-GX) & HUMAN VOLUNTEER, $127, \mathrm{M}$ \\
\hline LX3961 & FRONTAL & $0^{\circ}$ REARWARD (-GX) & HUMAN VOLUNTEER, $134, \mathrm{M}$ \\
\hline LX3962 & FRONTAL & $0^{\circ}$ REARWARD (-GX) & HUMAN VOLUNTEER, 136, M \\
\hline LX3963 & FRONTAL & $0^{\circ}$ REARWARD (-GX) & HUMAN VOLUNTEER, 133, M \\
\hline LX3965 & FRONTAL & $0^{\circ}$ REARWARD (-GX) & HUMAN VOLUNTEER, 135, M \\
\hline LX3968 & FRONTAL & $0^{\circ}$ REARWARD (-GX) & HUMAN VOLUNTEER, 134, M \\
\hline LX3969 & FRONTAL & $0^{\circ}$ REARWARD (-GX) & HUMAN VOLUNTEER, $118, \mathrm{M}$ \\
\hline LX3970 & FRONTAL & $0^{\circ}$ REARWARD (-GX) & HUMAN VOLUNTEER, $135, \mathrm{M}$ \\
\hline LX3972 & FRONTAL & $0^{\circ}$ REARWARD (-GX) & HUMAN VOLUNTEER, 120, M \\
\hline LX3982 & FRONTAL & $0^{\circ}$ REARWARD (-GX) & HUMAN VOLUNTEER, $132, \mathrm{M}$ \\
\hline LX3983 & FRONTAL & $0^{\circ}$ REARWARD (-GX) & HUMAN VOLUNTEER, $134, \mathrm{M}$ \\
\hline LX3985 & FRONTAL & $0^{\circ}$ REARWARD (-GX) & HUMAN VOLUNTEER, $118, \mathrm{M}$ \\
\hline LX3986 & FRONTAL & $0^{\circ}$ REARWARD (-GX) & HUMAN VOLUNTEER, $133, \mathrm{M}$ \\
\hline LX3987 & FRONTAL & $0^{\circ}$ REARWARD (-GX) & HUMAN VOLUNTEER, 131, M \\
\hline LX3989 & FRONTAL & $0^{\circ}$ REARWARD (-GX) & HUMAN VOLUNTEER, $132, \mathrm{M}$ \\
\hline LX3990 & FRONTAL & $0^{\circ}$ REARWARD (-GX) & HUMAN VOLUNTEER, 131, M \\
\hline LX3991 & FRONTAL & $0^{\circ}$ REARWARD (-GX) & HUMAN VOLUNTEER, 130, M \\
\hline LX3993 & FRONTAL & $0^{\circ}$ REARWARD (-GX) & HUMAN VOLUNTEER, $134, \mathrm{M}$ \\
\hline LX3994 & FRONTAL & $0^{\circ}$ REARWARD (-GX) & HUMAN VOLUNTEER, 135, M \\
\hline LX3995 & FRONTAL & $0^{\circ}$ REARWARD (-GX) & HUMAN VOLUNTEER, $120, \mathrm{M}$ \\
\hline LX3997 & FRONTAL & $0^{\circ}$ REARWARD (-GX) & HUMAN VOLUNTEER, 132, M \\
\hline LX3998 & FRONTAL & $0^{\circ}$ REARWARD (-GX) & HUMAN VOLUNTEER, $133, \mathrm{M}$ \\
\hline LX3999 & FRONTAL & $0^{\circ}$ REARWARD (-GX) & HUMAN VOLUNTEER, $131, \mathrm{M}$ \\
\hline LX4050 & SIDE & $90^{\circ}$ LATERAL $(+\mathrm{GY})$ & HUMAN VOLUNTEER, 130, M \\
\hline LX4052 & SIDE & $90^{\circ}$ LATERAL $(+\mathrm{GY})$ & HUMAN VOLUNTEER, 131, M \\
\hline LX4053 & SIDE & $90^{\circ}$ LATERAL (+GY) & HUMAN VOLUNTEER, 132, M \\
\hline LX4054 & SIDE & $90^{\circ}$ LATERAL $(+\mathrm{GY})$ & HUMAN VOLUNTEER, 134, M \\
\hline LX4055 & SIDE & $90^{\circ}$ LATERAL $(+\mathrm{GY})$ & HUMAN VOLUNTEER, $135, \mathrm{M}$ \\
\hline LX4057 & SIDE & $90^{\circ}$ LATERAL $(+\mathrm{GY})$ & HUMAN VOLUNTEER, 133, M \\
\hline LX4058 & SIDE & $90^{\circ}$ LATERAL $(+\mathrm{GY})$ & HUMAN VOLUNTEER, $136, \mathrm{M}$ \\
\hline LX4059 & SIDE & $90^{\circ}$ LATERAL $(+\mathrm{GY})$ & HUMAN VOLUNTEER, 138, M \\
\hline LX4060 & SIDE & $90^{\circ}$ LATERAL $(+\mathrm{GY})$ & HUMAN VOLUNTEER, $140, \mathrm{M}$ \\
\hline LX4068 & SIDE & $90^{\circ}$ LATERAL $(+\mathrm{GY})$ & HUMAN VOLUNTEER, $141, \mathrm{M}$ \\
\hline LX4069 & SIDE & $90^{\circ}$ LATERAL $(+\mathrm{GY})$ & HUMAN VOLUNTEER, 139, M \\
\hline LX4070 & SIDE & $90^{\circ}$ LATERAL $(+\mathrm{GY})$ & HUMAN VOLUNTEER, $130, \mathrm{M}$ \\
\hline LX4071 & SIDE & $90^{\circ}$ LATERAL $(+\mathrm{GY})$ & HUMAN VOLUNTEER, 131, M \\
\hline LX4073 & SIDE & $90^{\circ}$ LATERAL $(+\mathrm{GY})$ & HUMAN VOLUNTEER, $142, \mathrm{M}$ \\
\hline LX4074 & SIDE & $90^{\circ}$ LATERAL $(+\mathrm{GY})$ & HUMAN VOLUNTEER, 132, M \\
\hline LX4075 & SIDE & $90^{\circ}$ LATERAL $(+\mathrm{GY})$ & HUMAN VOLUNTEER, 133, M \\
\hline LX4076 & SIDE & $90^{\circ}$ LATERAL $(+\mathrm{GY})$ & HUMAN VOLUNTEER, $134, \mathrm{M}$ \\
\hline LX4078 & SIDE & $90^{\circ}$ LATERAL $(+\mathrm{GY})$ & HUMAN VOLUNTEER, 135, M \\
\hline LX4079 & SIDE & $90^{\circ}$ LATERAL $(+\mathrm{GY})$ & HUMAN VOLUNTEER, $136, \mathrm{M}$ \\
\hline LX4080 & SIDE & $90^{\circ}$ LATERAL $(+\mathrm{GY})$ & HUMAN VOLUNTEER, 138, M \\
\hline LX4081 & SIDE & $90^{\circ}$ LATERAL $(+\mathrm{GY})$ & HUMAN VOLUNTEER, $140, \mathrm{M}$ \\
\hline LX4083 & SIDE & $90^{\circ}$ LATERAL $(+\mathrm{GY})$ & HUMAN VOLUNTEER, $141, \mathrm{M}$ \\
\hline LX4084 & SIDE & $90^{\circ}$ LATERAL (+GY) & HUMAN VOLUNTEER, 142, M \\
\hline LX4085 & SIDE & $90^{\circ}$ LATERAL $(+\mathrm{GY})$ & HUMAN VOLUNTEER, $139, \mathrm{M}$ \\
\hline
\end{tabular}




\begin{tabular}{|c|c|c|c|}
\hline LX4088 & SIDE & $90^{\circ}$ LATERAL $(+\mathrm{GY})$ & HUMAN VOLUNTEER, 130, M \\
\hline LX4089 & SIDE & 90 LATERAL (+GY) & HUMAN VOLUNTEER, 131, M \\
\hline LX4090 & SIDE & $90^{\circ}$ LATERAL $(+\mathrm{GY})$ & HUMAN VOLUNTEER, 132, M \\
\hline LX4092 & SIDE & $90^{\circ}$ LATERAL $(+\mathrm{GY})$ & HUMAN VOLUNTEER, 138, M \\
\hline LX4093 & SIDE & $90^{\circ}$ LATERAL $(+\mathrm{GY})$ & HUMAN VOLUNTEER, 133, M \\
\hline LX4094 & SIDE & $90^{\circ}$ LATERAL $(+\mathrm{GY})$ & HUMAN VOLUNTEER, 141, M \\
\hline LX4095 & SIDE & $90^{\circ}$ LATERAL $(+\mathrm{GY})$ & HUMAN VOLUNTEER, 135, M \\
\hline LX4097 & SIDE & $90^{\circ}$ LATERAL $(+\mathrm{GY})$ & HUMAN VOLUNTEER, 134, M \\
\hline LX4098 & SIDE & $90^{\circ}$ LATERAL $(+\mathrm{GY})$ & HUMAN VOLUNTEER, $136, \mathrm{M}$ \\
\hline LX4099 & SIDE & $90^{\circ}$ LATERAL $(+\mathrm{GY})$ & HUMAN VOLUNTEER, 140, M \\
\hline LX4100 & SIDE & $90^{\circ}$ LATERAL $(+\mathrm{GY})$ & HUMAN VOLUNTEER, $139, \mathrm{M}$ \\
\hline LX4104 & SIDE & $90^{\circ}$ LATERAL $(+\mathrm{GY})$ & HUMAN VOLUNTEER, 142, M \\
\hline LX4107 & SIDE & $90^{\circ}$ LATERAL $(+\mathrm{GY})$ & HUMAN VOLUNTEER, 130, M \\
\hline LX4109 & SIDE & $90^{\circ}$ LATERAL $(+\mathrm{GY})$ & HUMAN VOLUNTEER, 131, M \\
\hline LX4110 & SIDE & $90^{\circ}$ LATERAL $(+\mathrm{GY})$ & HUMAN VOLUNTEER, 132, M \\
\hline LX4111 & SIDE & $90^{\circ}$ LATERAL $(+\mathrm{GY})$ & HUMAN VOLUNTEER, $133, \mathrm{M}$ \\
\hline LX4112 & SIDE & $90^{\circ}$ LATERAL $(+\mathrm{GY})$ & HUMAN VOLUNTEER, 134, M \\
\hline LX4114 & SIDE & $90^{\circ}$ LATERAL $(+\mathrm{GY})$ & HUMAN VOLUNTEER, 135, M \\
\hline LX4115 & SIDE & $90^{\circ}$ LATERAL $(+\mathrm{GY})$ & HUMAN VOLUNTEER, $138, \mathrm{M}$ \\
\hline LX4116 & SIDE & $90^{\circ}$ LATERAL $(+\mathrm{GY})$ & HUMAN VOLUNTEER, 140, M \\
\hline LX4118 & SIDE & $90^{\circ}$ LATERAL $(+\mathrm{GY})$ & HUMAN VOLUNTEER, $139, \mathrm{M}$ \\
\hline LX4120 & SIDE & $90^{\circ}$ LATERAL $(+\mathrm{GY})$ & HUMAN VOLUNTEER, 142, M \\
\hline LX4123 & SIDE & $90^{\circ}$ LATERAL $(+\mathrm{GY})$ & HUMAN VOLUNTEER, 130, M \\
\hline LX4124 & SIDE & $90^{\circ}$ LATERAL $(+\mathrm{GY})$ & HUMAN VOLUNTEER, 131, M \\
\hline LX4125 & SIDE & $90^{\circ}$ LATERAL $(+\mathrm{GY})$ & HUMAN VOLUNTEER, $133, \mathrm{M}$ \\
\hline LX4126 & SIDE & $90^{\circ}$ LATERAL $(+\mathrm{GY})$ & HUMAN VOLUNTEER, $134, \mathrm{M}$ \\
\hline LX4128 & SIDE & $90^{\circ}$ LATERAL $(+\mathrm{GY})$ & HUMAN VOLUNTEER, 132, M \\
\hline LX4129 & SIDE & $90^{\circ}$ LATERAL $(+\mathrm{GY})$ & HUMAN VOLUNTEER, $138, \mathrm{M}$ \\
\hline LX4130 & SIDE & $90^{\circ}$ LATERAL $(+\mathrm{GY})$ & HUMAN VOLUNTEER, 140, M \\
\hline LX4131 & SIDE & $90^{\circ}$ LATERAL $(+\mathrm{GY})$ & HUMAN VOLUNTEER, 135, M \\
\hline LX4133 & SIDE & $90^{\circ}$ LATERAL $(+\mathrm{GY})$ & HUMAN VOLUNTEER, 139, M \\
\hline LX4134 & SIDE & $90^{\circ}$ LATERAL $(+\mathrm{GY})$ & HUMAN VOLUNTEER, 141, M \\
\hline LX4135 & SIDE & $90^{\circ}$ LATERAL $(+\mathrm{GY})$ & HUMAN VOLUNTEER, $142, \mathrm{M}$ \\
\hline LX4137 & SIDE & $90^{\circ}$ LATERAL $(+\mathrm{GY})$ & HUMAN VOLUNTEER, 130, M \\
\hline LX4138 & SIDE & $90^{\circ}$ LATERAL $(+\mathrm{GY})$ & HUMAN VOLUNTEER, $131, \mathrm{M}$ \\
\hline LX4139 & SIDE & $90^{\circ}$ LATERAL (+GY) & HUMAN VOLUNTEER, 134, M \\
\hline LX4140 & SIDE & $90^{\circ}$ LATERAL $(+\mathrm{GY})$ & HUMAN VOLUNTEER, 135, M \\
\hline LX4142 & SIDE & $90^{\circ}$ LATERAL $(+\mathrm{GY})$ & HUMAN VOLUNTEER, $136, \mathrm{M}$ \\
\hline LX4143 & SIDE & $90^{\circ}$ LATERAL $(+\mathrm{GY})$ & HUMAN VOLUNTEER, 132, M \\
\hline LX4144 & SIDE & $90^{\circ}$ LATERAL $(+\mathrm{GY})$ & HUMAN VOLUNTEER, 139, M \\
\hline LX4145 & SIDE & $90^{\circ}$ LATERAL (+GY) & HUMAN VOLUNTEER, 140, M \\
\hline LX4147 & SIDE & $90^{\circ}$ LATERAL $(+\mathrm{GY})$ & HUMAN VOLUNTEER, 138, M \\
\hline LX4148 & SIDE & $90^{\circ}$ LATERAL $(+\mathrm{GY})$ & HUMAN VOLUNTEER, $141, \mathrm{M}$ \\
\hline LX4149 & SIDE & $90^{\circ}$ LATERAL $(+\mathrm{GY})$ & HUMAN VOLUNTEER, 142, M \\
\hline LX4151 & SIDE & $90^{\circ}$ LATERAL $(+\mathrm{GY})$ & HUMAN VOLUNTEER, $133, \mathrm{M}$ \\
\hline LX4153 & SIDE & $90^{\circ}$ LATERAL $(+\mathrm{GY})$ & HUMAN VOLUNTEER, 136, M \\
\hline LX4155 & SIDE & $90^{\circ}$ LATERAL (+GY) & HUMAN VOLUNTEER, 132, M \\
\hline LX4159 & OBLIQUE & $45^{\circ}$ OBLIQUE $(-\mathrm{GX},+\mathrm{GY})$ & HUMAN VOLUNTEER, $130, \mathrm{M}$ \\
\hline LX4161 & OBLIQUE & $45^{\circ}$ OBLIQUE (-GX, +GY) & HUMAN VOLUNTEER, 131, M \\
\hline LX4162 & OBLIQUE & $45^{\circ}$ OBLIQUE $(-\mathrm{GX},+\mathrm{GY})$ & HUMAN VOLUNTEER, $132, \mathrm{M}$ \\
\hline LX4163 & OBLIQUE & $45^{\circ}$ OBLIQUE $(-\mathrm{GX},+\mathrm{GY})$ & HUMAN VOLUNTEER, 133, M \\
\hline LX4164 & OBLIQUE & $45^{\circ}$ OBLIQUE (-GX, +GY) & HUMAN VOLUNTEER, 134, M \\
\hline LX4166 & OBLIQUE & $45^{\circ}$ OBLIQUE $(-\mathrm{GX},+\mathrm{GY})$ & HUMAN VOLUNTEER, $135, \mathrm{M}$ \\
\hline LX4167 & OBLIQUE & $45^{\circ}$ OBLIQUE (-GX, +GY) & HUMAN VOLUNTEER, 136, M \\
\hline LX4168 & OBLIQUE & $45^{\circ}$ OBLIQUE $(-\mathrm{GX},+\mathrm{GY})$ & HUMAN VOLUNTEER, $138, \mathrm{M}$ \\
\hline LX4170 & OBLIQUE & $45^{\circ}$ OBLIQUE (-GX, +GY) & HUMAN VOLUNTEER, 139, M \\
\hline LX4171 & OBLIQUE & $45^{\circ}$ OBLIQUE $(-\mathrm{GX},+\mathrm{GY})$ & HUMAN VOLUNTEER, $141, \mathrm{M}$ \\
\hline LX4172 & OBLIQUE & $45^{\circ}$ OBLIQUE (-GX, +GY) & HUMAN VOLUNTEER, 142, M \\
\hline LX4234 & OBLIQUE & $45^{\circ}$ OBLIQUE (-GX, +GY) & HUMAN VOLUNTEER, 140, M \\
\hline LX4235 & OBLIQUE & $45^{\circ}$ OBLIQUE $(-\mathrm{GX},+\mathrm{GY})$ & HUMAN VOLUNTEER, $130, \mathrm{M}$ \\
\hline LX4236 & OBLIQUE & $45^{\circ}$ OBLIQUE (-GX, +GY) & HUMAN VOLUNTEER, 133, M \\
\hline LX4237 & OBLIQUE & $45^{\circ}$ OBLIQUE (-GX, +GY) & HUMAN VOLUNTEER, 134, M \\
\hline
\end{tabular}




\begin{tabular}{|c|c|c|c|}
\hline LX4238 & OBLIQUE & $45^{\circ}$ OBLIQUE $(-\mathrm{GX},+\mathrm{GY})$ & HUMAN VOLUNTEER, 135, M \\
\hline LX4240 & OBLIQUE & $45^{\circ}$ OBLIQUE $(-\mathrm{GX},+\mathrm{GY})$ & HUMAN VOLUNTEER, 133, M \\
\hline LX4241 & OBLIQUE & $45^{\circ}$ OBLIQUE $(-\mathrm{GX},+\mathrm{GY})$ & HUMAN VOLUNTEER, $138, \mathrm{M}$ \\
\hline LX4242 & OBLIQUE & $45^{\circ}$ OBLIQUE (-GX, +GY) & HUMAN VOLUNTEER, 131, M \\
\hline LX4243 & OBLIQUE & $45^{\circ}$ OBLIQUE $(-\mathrm{GX},+\mathrm{GY})$ & HUMAN VOLUNTEER, $139, \mathrm{M}$ \\
\hline LX4244 & OBLIQUE & $45^{\circ}$ OBLIQUE (-GX, +GY) & HUMAN VOLUNTEER, 132, M \\
\hline LX4246 & OBLIQUE & $45^{\circ}$ OBLIQUE $(-\mathrm{GX},+\mathrm{GY})$ & HUMAN VOLUNTEER, $131, \mathrm{M}$ \\
\hline LX4247 & OBLIQUE & $45^{\circ}$ OBLIQUE $(-\mathrm{GX},+\mathrm{GY})$ & HUMAN VOLUNTEER, 136, M \\
\hline LX4248 & OBLIQUE & $45^{\circ}$ OBLIQUE $(-\mathrm{GX},+\mathrm{GY})$ & HUMAN VOLUNTEER, $141, \mathrm{M}$ \\
\hline LX4249 & OBLIQUE & $45^{\circ}$ OBLIQUE $(-\mathrm{GX},+\mathrm{GY})$ & HUMAN VOLUNTEER, 142, M \\
\hline LX4251 & OBLIQUE & $45^{\circ}$ OBLIQUE $(-\mathrm{GX},+\mathrm{GY})$ & HUMAN VOLUNTEER, $131, \mathrm{M}$ \\
\hline LX4259 & OBLIQUE & $45^{\circ}$ OBLIQUE $(-\mathrm{GX},+\mathrm{GY})$ & HUMAN VOLUNTEER, $140, \mathrm{M}$ \\
\hline LX4260 & OBLIQUE & $45^{\circ}$ OBLIQUE $(-\mathrm{GX},+\mathrm{GY})$ & HUMAN VOLUNTEER, $130, \mathrm{M}$ \\
\hline LX4261 & OBLIQUE & $45^{\circ}$ OBLIQUE (-GX, +GY) & HUMAN VOLUNTEER, $132, \mathrm{M}$ \\
\hline LX4263 & OBLIQUE & $45^{\circ}$ OBLIQUE $(-\mathrm{GX},+\mathrm{GY})$ & HUMAN VOLUNTEER, $136, \mathrm{M}$ \\
\hline LX4264 & OBLIQUE & $45^{\circ}$ OBLIQUE (-GX, +GY) & HUMAN VOLUNTEER, $134, \mathrm{M}$ \\
\hline LX4265 & OBLIQUE & $45^{\circ}$ OBLIQUE $(-\mathrm{GX},+\mathrm{GY})$ & HUMAN VOLUNTEER, $138, \mathrm{M}$ \\
\hline LX4266 & OBLIQUE & $45^{\circ}$ OBLIQUE (-GX, +GY) & HUMAN VOLUNTEER, 135, M \\
\hline LX4268 & OBLIQUE & $45^{\circ}$ OBLIQUE $(-\mathrm{GX},+\mathrm{GY})$ & HUMAN VOLUNTEER, $139, \mathrm{M}$ \\
\hline LX4269 & OBLIQUE & $45^{\circ}$ OBLIQUE $(-\mathrm{GX},+\mathrm{GY})$ & HUMAN VOLUNTEER, 140, M \\
\hline LX4270 & OBLIQUE & $45^{\circ}$ OBLIQUE $(-\mathrm{GX},+\mathrm{GY})$ & HUMAN VOLUNTEER, $141, \mathrm{M}$ \\
\hline LX4271 & OBLIQUE & $45^{\circ}$ OBLIQUE $(-\mathrm{GX},+\mathrm{GY})$ & HUMAN VOLUNTEER, $142, \mathrm{M}$ \\
\hline LX4281 & OBLIQUE & $45^{\circ}$ OBLIQUE $(-\mathrm{GX},+\mathrm{GY})$ & HUMAN VOLUNTEER, $140, \mathrm{M}$ \\
\hline LX4282 & OBLIQUE & $45^{\circ}$ OBLIQUE $(-\mathrm{GX},+\mathrm{GY})$ & HUMAN VOLUNTEER, 141, M \\
\hline LX4284 & OBLIQUE & $45^{\circ}$ OBLIQUE $(-\mathrm{GX},+\mathrm{GY})$ & HUMAN VOLUNTEER, $138, \mathrm{M}$ \\
\hline LX4286 & OBLIQUE & $45^{\circ}$ OBLIQUE $(-\mathrm{GX},+\mathrm{GY})$ & HUMAN VOLUNTEER, $130, \mathrm{M}$ \\
\hline LX4287 & OBLIQUE & $45^{\circ}$ OBLIQUE $(-\mathrm{GX},+\mathrm{GY})$ & HUMAN VOLUNTEER, $132, \mathrm{M}$ \\
\hline LX4288 & OBLIQUE & $45^{\circ}$ OBLIQUE (-GX, +GY) & HUMAN VOLUNTEER, 142, M \\
\hline LX4290 & OBLIQUE & $45^{\circ}$ OBLIQUE $(-\mathrm{GX},+\mathrm{GY})$ & HUMAN VOLUNTEER, $134, \mathrm{M}$ \\
\hline LX4291 & OBLIQUE & $45^{\circ}$ OBLIQUE $(-\mathrm{GX},+\mathrm{GY})$ & HUMAN VOLUNTEER, 139, M \\
\hline LX4292 & OBLIQUE & $45^{\circ}$ OBLIQUE $(-\mathrm{GX},+\mathrm{GY})$ & HUMAN VOLUNTEER, $141, \mathrm{M}$ \\
\hline LX4293 & OBLIQUE & $45^{\circ}$ OBLIQUE $(-\mathrm{GX},+\mathrm{GY})$ & HUMAN VOLUNTEER, $140, \mathrm{M}$ \\
\hline LX4295 & OBLIQUE & $45^{\circ}$ OBLIQUE $(-\mathrm{GX},+\mathrm{GY})$ & HUMAN VOLUNTEER, $142, \mathrm{M}$ \\
\hline LX4296 & OBLIQUE & $45^{\circ}$ OBLIQUE $(-\mathrm{GX},+\mathrm{GY})$ & HUMAN VOLUNTEER, $138, \mathrm{M}$ \\
\hline LX4297 & OBLIQUE & $45^{\circ}$ OBLIQUE $(-\mathrm{GX},+\mathrm{GY})$ & HUMAN VOLUNTEER, $132, \mathrm{M}$ \\
\hline LX4298 & OBLIQUE & $45^{\circ}$ OBLIQUE $(-\mathrm{GX},+\mathrm{GY})$ & HUMAN VOLUNTEER, $134, \mathrm{M}$ \\
\hline LX4301 & OBLIQUE & $45^{\circ}$ OBLIQUE $(-\mathrm{GX},+\mathrm{GY})$ & HUMAN VOLUNTEER, $130, \mathrm{M}$ \\
\hline LX4302 & OBLIQUE & $45^{\circ}$ OBLIQUE (-GX, +GY) & HUMAN VOLUNTEER, $140, \mathrm{M}$ \\
\hline LX4303 & OBLIQUE & $45^{\circ}$ OBLIQUE $(-\mathrm{GX},+\mathrm{GY})$ & HUMAN VOLUNTEER, $139, \mathrm{M}$ \\
\hline LX4305 & OBLIQUE & $45^{\circ}$ OBLIQUE $(-\mathrm{GX},+\mathrm{GY})$ & HUMAN VOLUNTEER, 138, M \\
\hline LX4306 & OBLIQUE & $45^{\circ}$ OBLIQUE $(-\mathrm{GX},+\mathrm{GY})$ & HUMAN VOLUNTEER, $132, \mathrm{M}$ \\
\hline LX4307 & OBLIQUE & $45^{\circ}$ OBLIQUE $(-\mathrm{GX},+\mathrm{GY})$ & HUMAN VOLUNTEER, 134, M \\
\hline LX4309 & OBLIQUE & $45^{\circ}$ OBLIQUE $(-\mathrm{GX},+\mathrm{GY})$ & HUMAN VOLUNTEER, $130, \mathrm{M}$ \\
\hline LX4310 & OBLIQUE & $45^{\circ}$ OBLIQUE $(-\mathrm{GX},+\mathrm{GY})$ & HUMAN VOLUNTEER, $140, \mathrm{M}$ \\
\hline LX4313 & OBLIQUE & $45^{\circ}$ OBLIQUE $(-\mathrm{GX},+\mathrm{GY})$ & HUMAN VOLUNTEER, $139, \mathrm{M}$ \\
\hline LX4314 & OBLIQUE & $45^{\circ}$ OBLIQUE $(-\mathrm{GX},+\mathrm{GY})$ & HUMAN VOLUNTEER, $135, \mathrm{M}$ \\
\hline LX4316 & OBLIQUE & $45^{\circ}$ OBLIQUE $(-\mathrm{GX},+\mathrm{GY})$ & HUMAN VOLUNTEER, $135, \mathrm{M}$ \\
\hline
\end{tabular}




\section{References}

Abel, J.M., Gennarelli, T.A., \& Segawa, H. (1978). Incidence and severity of cerebral concussion in the rhesus monkey following sagittal plane angular acceleration (No. 780886). SAE Technical Paper.

Allison, M.A., Kang, Y.S., Bolte, J.H., Maltese, M.R., Arbogast, K.B. (2014). Validation of a helmet-based system to measure head impact biomechanics in ice hockey. Medicine and Science in Sports and Exercise, 46(1), 115-123.

Allison, M.A., Kang, Y.S., Maltese, M.R., Bolte IV, J.H., \& Arbogast, K.B. (2015). Measurement of Hybrid III head impact kinematics using an accelerometer and gyroscope system in ice hockey helmets. Annals of Biomedical Engineering, 43(8), 1896-1906.

Alshareef, A., Giudice, J.S., Forman, J., Salzar, R.S., \& Panzer, M.B. (2017). A novel method for quantifying human in situ whole brain deformation under rotational loading using sonomicrometry. Journal of Neurotrauma, (ja).

Antona-Makoshi, J. (2013). Reanalysis of primate head impact experiments to clarify mild traumatic brain injury kinematics and thresholds. Ph.D. Thesis. ISSN 1652-8565.

Arbogast, K.B., \& Margulies, S.S. (1998). Material characterization of the brainstem from oscillatory shear tests. Journal of Biomechanics, 31(9), 801-807.

Azevedo, F.A.C., Carvalho, L.R.B., Grinberg, L.T., Farfel, J.M., Ferretti, R.E.L., Leite, R.E.P., Filho, W.J., Lent, R., \& Herculano-Houzel, S. (2009). Equal numbers of neuronal and nonneuronal cells make the human brain an isometrically scaled-up primate brain. Journal of Comparative Neurology, 513(5), 532-541.

Bain, A.C., \& Meaney, D.F. (2000). Tissue-level thresholds for axonal damage in an experimental model of central nervous system white matter injury. Journal of Biomechanical Engineering, 122(6), 615-622.

Bandak, F.A. (1995). On the mechanics of impact neurotrauma: a review and critical synthesis. Journal of Neurotrauma, 12(4), 635-649.

Bandak, F.A., Zhang, A.X., Tannous, R.E., DiMasi, F., Masiello, P., \& Eppinger, R.H. (2001). Simon: a simulated injury monitor; application to head injury assessment. In $17^{\text {th }}$ Technical Conference on the Enhanced Safety of Vehicles.

Bartsch, A., Samorezov, S., Benzel, E., Miele, V., \& Brett, D. (2014). Validation of an "intelligent mouthguard" single event head impact dosimeter. Stapp Car Crash J., 58, 1.

Brinkley, J.W., \& Shaffer, J.T. (1971). Dynamic simulation techniques for the design of escape systems: current applications and future air force requirements (No. AMRL-TR-7129-PAPER-2). Air Force Aerospace Medical Research Lab, Wright-Patterson AFB, OH. 
Brinn, J., \& Staffeld, S.E. (1970). Evaluation of impact test accelerations: a damage index for the head and torso (No. 700902). SAE Technical Paper.

Camarillo, D.B., Shull, P.B., Mattson, J., Shultz, R., \& Garza, D. (2013). An instrumented mouthguard for measuring linear and angular head impact kinematics in American football. Annals of Biomedical Engineering, 41(9), 1939-1949.

Cater, H.L., Sundstrom, L.E., \& Morrison, B. (2006). Temporal development of hippocampal cell death is dependent on tissue strain but not strain rate. Journal of Biomechanics, 39(15), 2810-2818.

Coronado, V.G., McGuire, L.C., Sarmiento, K., Bell, J., Lionbarger, M.R., Jones, C.D., Geller, A.I., Khoury, N., \& Xu, L. (2012). Trends in traumatic brain injury in the US and the public health response: 1995-2009. Journal of Safety Research, 43(4), 299-307.

Crandall, J. (2013). Injury criteria development: THOR metric SD-3 shoulder advanced frontal crash test dummy. NHTSA Biomechanics Test Database.

Crandall, J. (2014). ATD thoracic response test development: $20^{\circ}$ far side oblique frontal, front passenger position. NHTSA Biomechanics Test Database.

Cullen, D.K., Simon, C.M., \& LaPlaca, M.C. (2007). Strain rate-dependent induction of reactive astrogliosis and cell death in three-dimensional neuronal-astrocytic co-cultures. Brain Research, 1158, 103-115.

Daneshvar, D.H., Nowinski, C.J., McKee, A., \& Cantu, R.C. (2011). The epidemiology of sport-related concussion. Clinics in Sports Medicine, 30(1), 1-17.

Darvish, K.K., \& Crandall, J.R. (2001). Nonlinear viscoelastic effects in oscillatory shear deformation of brain tissue. Medical Engineering \& Physics, 23(9), 633-645.

Deck, C., \& Willinger, R. (2009). The current state of the human head finite element modelling. International Journal of Vehicle Safety, 4(2), 85-112.

Denny-Brown, D., \& Russell, W.R. (1941). Experimental cerebral concussion. Brain, 64(2-3), 93-164.

Department of Transportation NHTSA Docket Number 69-7, Notice 19 Occupant Crash Protection - Head Injury Criterion, S6.2 of MVSS 208.

Duma, S.M., Manoogian, S.J., Bussone, W.R., Brolinson, P.G., Goforth, M.W., Donnenwerth, J.J., Greenwald, R.M., Chu, J.J., \& Crisco, J.J. (2005). Analysis of real-time head accelerations in collegiate football players. Clinical J. Sport Medicine, 15(1), 3-8.

Eigen, A.M., \& Martin, P.G. (2005). Identification of real world injury patterns in aid of dummy development. In $19^{\text {th }}$ Technical Conference on the Enhanced Safety of Vehicles. 
Elkin, B.S., \& Morrison III, B. (2007). Region-specific tolerance criteria for the living brain. Stapp Car Crash Journal, 51, 127-138.

Elkin, B.S., Elliott, J.M., \& Siegmund, G.P. (2016). Whiplash injury or concussion? A possible biomechanical explanation for concussion symptoms in some individuals following a rear-end collision. J. Orth. \& Sports Physical Therapy, 46(10), 874-885.

Elliott, M.R., Margulies, S.S., Maltese, M.R., \& Arbogast, K.B. (2015). Accounting for sampling variability, injury under-reporting, and sensor error in concussion injury risk curves. Journal of Biomechanics, 48(12), 3059-3065.

Ewing, C.L., \& Thomas, D.J. (1972). Human head and neck response to impact acceleration (No. NAMRL-MONOGRAPH-21). Naval Aerospace Medical Research Lab, Pensacola, FL.

Fan, W.R. (1971). Internal head injury assessment (No. 710870). SAE Technical Paper.

Finan, J.D., Sundaresh, S.N., Elkin, B.S., McKhann, G.M., \& Morrison, B. (2017). Regional mechanical properties of human brain tissue for computational models of traumatic brain injury. Acta Biomaterialia, 55, 333-339.

Forman J.L., Lopez-Valdes F., Lessley D.J., Riley P., Sochor M., Heltzel S., Ash J., Perz R., Kent R.W., Seacrist, T. \& Arbogast, K.B. (2013). Occupant kinematics and shoulder belt retention in far-side lateral and oblique collisions: a parametric study. Stapp Car Crash Journal, 57, 343-385.

Fountoulakis, M., Hardmeier, R., Höger, H., \& Lubec, G. (2001). Postmortem changes in the level of brain proteins. Experimental Neurology, 167(1), 86-94.

Gabler, L.F., Crandall, J.R., \& Panzer, M.B. (2016). Investigating brain injury tolerance in the sagittal plane using a finite element model of the human head. International Journal of Automotive Engineering, 7(1), 37-43.

Gadd, C.W. (1966). Use of a weighted-impulse criterion for estimating injury hazard (No. 660793). SAE Technical Paper.

Gefen, A., Gefen, N., Zhu, Q., Raghupathi, R., \& Margulies, S.S. (2003). Age-dependent changes in material properties of the brain and braincase of the rat. Journal of Neurotrauma, 20(11), 1163-1177.

Gennarelli, T.A. (1993). Cerebral concussion and diffuse brain injuries. Head Injury, 2, $108-124$.

Gennarelli, T.A., Thibault, L.E., \& Omaya, A.K. (1971). Comparison of translational and rotational head motions in experimental cerebral concussion. In $15^{\text {th }}$ Stapp Car Crash Conference. 
Gennarelli, T.A., Abel, J.M., Adams, H., \& Graham, D. (1979). Differential tolerance of frontal and temporal lobes to contusion induced by angular acceleration (No. 791022). SAE Technical Paper.

Gennarelli, T.A., Thibault, L.E., Adams, J.H., Graham, D.I., Thompson, C.J., \& Marcincin, R.P. (1982). Diffuse axonal injury and traumatic coma in the primate. Annals of Neurology, 12(6), 564-574.

Gennarelli, T.A., Thibault, L.E., \& Wilkins, R.H. (1985). Biomechanics of head injury. Neurosurgery, 2, 1531-1536.

Gennarelli, T.A., Thibault, L.E., \& Graham, D.I. (1998). Diffuse axonal injury: an important form of traumatic brain damage. The Neuroscientist, 4(3), 202-215.

Gennarelli, T.A. (2015). What do we know about angular head motions (AHM)? Presented at the IRCOBI-NOCSAE-Snell-PDB TBI Workshop. Lyon, France.

Gierke, von H.E. (1964). Transient acceleration, vibration and noise problems in space flight. Bioastronautics. New York. Macmillan, 27175.

Goggio, A.F. (1941). The mechanism of contre-coup injury. Journal of Neurology and Psychiatry, 4(1), 11.

Goldsmith, W. (1972). Biomechanics of head injury. Biomechanics: Its foundation and objectives, 585-634.

Greenwald, R.M., Gwin, J.T., Chu, J.J., \& Crisco, J.J. (2008). Head impact severity measures for evaluating mild traumatic brain injury risk exposure. Neurosurgery, 62(4), 789-798.

Hardy, W.N., Foster, C.D., Mason, M.J., Yang, K.H., King, A.I., \& Tashman, S. (2001). Investigation of head injury mechanisms using neutral density technology and high-speed biplanar x-ray. Stapp Car Crash Journal, 45, 337-368.

Hardy, W.N., Mason, M.J., Foster, C.D., Shah, C.S., Kopacz, J.M., Yang, K.H., King, A.I., Bishop, J., Bey, M., Anderst, W., \& Tashman, S. (2007). A study of the response of the human cadaver head to impact. Stapp Car Crash Journal, 51, 17.

Harmon, K.G., Drezner, J.A., Gammons, M., Guskiewicz, K.M., Halstead, M., Herring, S.A., Kutcher, J.S., Pana, A., Putukian, M., \& Roberts, W.O. (2013). American medical society for sports medicine position statement: concussion in sport. British Journal of Sports Medicine, 47(1), 15-26.

Hasija, V., Takhounts, E.G., \& Ridella, S.A. (2007). Computational analysis of real world crashes: a basis for accident reconstruction methodology. In $20^{\text {th }}$ International Technical Conference on the Enhanced Safety of Vehicles. 
Henderson, K., Bailey, A.M., Christopher, J., Brozoski, F., \& Salzar, R. (2013). Biomechanical response of the lower leg under high rate loading. In Proceedings of IRCOBI Conference.

Hernandez, F., Wu, L.C., Yip, M.C., Laksari, K., Hoffman, A.R., Lopez, J.R., Grant, G.A., Kleiven, S., \& Camarillo, D.B. (2015). Six degree-of-freedom measurements of human mild traumatic brain injury. Annals of Biomedical Engineering, 43(8), 1918-1934.

Hewitt, P. (1861). A system of surgery. Ed. by T. Holmes, 2, London.

Holbourn, A.H.S. (1943). Mechanics of head injuries. The Lancet, 242(6267), 438-441.

Hrapko, M., van Dommelen, J. a. W., Peters, G.W.M., \& Wismans, J.S.H.M. (2008). The influence of test conditions on characterization of the mechanical properties of brain tissue. Journal of Biomechanical Engineering, 130(3), 031003.

IIHS TechData. Insurance Institute for Highway Safety (techdata.iihs.org).

Ji, S., \& Zhao, W. (2015). A pre-computed brain response atlas for instantaneous strain estimation in contact sports. Annals of Biomedical Engineering, 43(8), 1877-1895.

Ji, S., Ghadyani, H., Bolander, R.P., Beckwith, J.G., Ford, J.C., McAllister, T.W., Flashman, L.A., Paulsen, K.D., Ernstrom, K., Jain, S., \& Raman, R. (2014). Parametric comparisons of intracranial mechanical responses from three validated finite element models of the human head. Annals of Biomedical Engineering, 42(1), 11-24.

Kameyama, M., Karibe, H., Onuma, T., \& Tominaga, T. (2008). Epidemiological study of head injury in Miyagi Neurotrauma Data Bank: Age, cause of injury, pathophysiology and outcome. Neurotraumatology, 31, 49-56.

Kang, Y.-S., Moorhouse, K., \& Bolte, J.H. (2011). Measurement of six degrees of freedom head kinematics in impact conditions employing six accelerometers and three angular rate sensors (6aw configuration). Journal of Biomechanical Engineering, 133(11), 111007.

Kerrigan, J., Arregui-Dalmases, C., \& Crandall, J. (2012). Assessment of pedestrian head impact dynamics in small sedan and large SUV collisions. International Journal of Crashworthiness, 17(3), 243-258.

Kerrigan, J.R., Crandall, J.R., \& Deng, B. (2008). A comparative analysis of the pedestrian injury risk predicted by mechanical impactors and post mortem human surrogates. Stapp Car Crash Journal, 52, 527-567.

Kim, T., Shin, J., Ye, X., Crandall, J., Knospe, C., \& Funk, J. (2013). Evaluation of methods for the development of representative responses and corridors from biomechanical data using mechanical models. International Journal of Crashworthiness, 18(6), 633-646.

Kimpara, H., \& Iwamoto, M. (2012). Mild Traumatic Brain Injury Predictors Based on Angular Accelerations During Impacts. Annals of Biomedical Eng., 40(1), 114-126. 
Kimpara, H., Nakahira, Y., Iwamoto, M., Miki, K., Ichihara, K., Kawano, S., \& Taguchi, T. (2006). Investigation of anteroposterior head-neck responses during severe frontal impacts using a brain-spinal cord complex FE model (No. 2006-22-0019). SAE Paper.

Kimpara, H., Nakahira, Y., Iwamoto, M., Rowson, S., \& Duma, S. (2011). Head injury prediction methods based on 6 degree of freedom head acceleration measurements during impact. International Journal of Automotive Engineering, 2(2), 13-19.

King, A.I., Yang, K.H., Zhang, L., Hardy, W., \& Viano, D.C. (2003). Is head injury caused by linear or angular acceleration. In Proceedings of IRCOBI Conference.

Kleiven, S. (2007). Predictors for traumatic brain injuries evaluated through accident reconstructions. Stapp Car Crash Journal, 51, 81-114.

Kornhauser, M., \& Lawton, R.W. (1961). Impact tolerance of mammals. Planetary and Space Science, 7, 386-394.

Kucera, K.L., Klossner, D., Colgate, B., \& Cantu, R.C. (2013). Annual survey of football injury research. National Center for Catastrophic Sport Injury Research.

Laituri, T.R., El-Jawahri, R.E., Henry, S., \& Sullivan, K. (2015). Field-based assessments of various AIS2+ head risk curves for frontal impact (No. 2015-01-1437). SAE Paper.

Laituri, T.R., Henry, S., Pline, K., Li, G., Frankstein, M., \& Weerappuli, P. (2016). New risk curves for NHTSA's Brain Injury Criterion (BrIC): derivations and assessments. Stapp Car Crash Journal, 60, 301-362.

Laksari, K., Wu, L.C., Kurt, M., Kuo, C., \& Camarillo, D.C. (2015). Resonance of human brain under head acceleration. Journal of The Royal Society Interface, 12(108), 20150331.

Langlois, J.A., Rutland-Brown, W., \& Wald, M.M. (2006). The epidemiology and impact of traumatic brain injury: a brief overview. J. Head Trauma Rehabil, 21(5), 375-378.

Lobdell, T.E., Kroell, C.K., Schneider, D.C., Hering, W.E., \& Nahum, A.M. (1973). Impact response of the human thorax. Human Impact Response, Springer, 201-245.

Lobo, B., Lin, R., Brown, D., Kim, T., \& Panzer, M. (2015). Predicting pedestrian injury metrics based on vehicle front-end design. International Conference on Internet of Vehicles. Springer, 114-126.

Mao, H., Zhang, L., Jiang, B., Genthikatti, V.V., Jin, X., Zhu, F., Makwana, R., Gill, A., Jandir, G., Singh, A., \& Yang, K.H. (2013). Development of a finite element human head model partially validated with thirty five experimental cases. Journal of Biomechanical Engineering, 135(1), 111002.

Margulies, S.S., \& Thibault, L.E. (1992). A proposed tolerance criterion for diffuse axonal injury in man. Journal of Biomechanics, 25(8), 917-923. 
Marjoux, D., Baumgartner, D., Deck, C., \& Willinger, R. (2008). Head injury prediction capability of the HIC, HIP, SIMon and ULP criteria. Accident Analysis \& Prevention, 40(3), 1135-1148.

McCrea, M., Kelly, J.P., Randolph, C., Kluge, J., Bartolic, E., Finn, G., \& Baxter, B. (1998). Standardized Assessment of Concussion (SAC): on-site mental status evaluation of the athlete. The Journal of Head Trauma Rehabilitation, 13(2), 27-35.

McCrea, M., Hammeke, T., Olsen, G., Leo, P., \& Guskiewicz, K. (2004). Unreported concussion in high school football players: implications for prevention. Clinical Journal of Sport Medicine, 14(1), 13-17.

McCrory, P.R., \& Berkovic, S.F. (2001). Concussion: The history of clinical and pathophysiological concepts and misconceptions. Neurology, 57(12), 2283-2289.

McCrory, P., Meeuwisse, W., Dvorak, J., Aubry, M., Bailes, J., Broglio, S., Cantu, R.C., Cassidy, D., Echemendia, R.J., \& Castellani, R.J. (2017). Consensus statement on concussion in sport - the $5^{\text {th }}$ international conference on concussion in sport held in Berlin, October 2016, British Journal of Sports Medicine.

McElhaney, J.H., Roberts, V.L., \& Hilyard, J.F. (1976). Handbook of human tolerance. Japan Automobile Research Institute, Incorporated (JARI).

Meaney, D.F., Smith, D., Ross, D.T., \& Gennarelli, T.A. (1993). Diffuse axonal injury in the miniature pig: biomechanical development and injury threshold. Crashworthiness and Occupant Protection in Transportation Systems, 25, 169-175.

Meaney, D.F., Smith, D.H., Shreiber, D.I., Bain, A.C., Miller, R.T., Ross, D.T., \& Gennarelli, T.A. (1995). Biomechanical analysis of experimental diffuse axonal injury. Journal of Neurotrauma, 12(4), 689-694.

Meaney, D.F., Morrison, B., \& Bass, C.D. (2014). The mechanics of traumatic brain injury: a review of what we know and what we need to know for reducing its societal burden. Journal of Biomechanical Engineering, 136(2), 021008.

Mertz, H.J., Irwin, A.L., \& Prasad, P. (2003). Biomechanical and scaling bases for frontal and side impact injury assessment reference values. Stapp Car Crash J, 47, 155-188.

Miller, L.E., Urban, J.E., \& Stitzel, J.D. (2017). Validation performance comparison for finite element models of the human brain. Computer Methods in Biomechanics and Biomedical Engineering, 20(12), 1273-1288.

Milton, S.J., \& Arnold, J.C. (2002). Introduction to probability and statistics principles and applications for engineering and the computing sciences. McGraw-Hill, Inc.

Morrison, B., Cater, H.L., Wang, C.C.-B., Thomas, F.C., Hung, C.T., Ateshian, G.A., \& Sundstrom, L.E. (2003). A tissue level tolerance criterion for living brain developed with an in vitro model of traumatic mechanical loading. Stapp Car Crash Journal, 47, 93-105. 
Mueller, B., MacAlister, A., Nolan, J., \& Zuby, D. (2015). Comparison of HIC and BRIC head injury risk in IIHS frontal crash tests to real-world head injuries. In $24^{\text {th }}$ International Technical Conference on the Enhanced Safety of Vehicles.

Nahum, A.M., Smith, R., \& Ward, C.C. (1977). Intracranial pressure dynamics during head impact (No. 770922). SAE Technical Paper.

National Operating Committee on the Standards for Athletic Equipment (NOCSAE) (2012). Standard Test Method and Equipment Used in Evaluating the Performance Characteristics of Protective Headgear/Equipment.

Newman, J. (1986). A generalized acceleration model for brain injury threshold (GAMBIT). In Proceedings of IRCOBI Conference.

Newman, J.A., Shewchenko, N., \& Welbourne, E. (2000). A proposed new biomechanical head injury assessment function - the maximum power index. Stapp Car Crash Journal, $44,215-247$.

NHTSA NVS | Vehicle Crash Test Database (nhtsa.gov).

NHTSA NVS | Biomechanics Test Database (nhtsa.gov).

NHTSA (2014). Traffic Safety Facts. National Highway Traffic Safety Administration.

NHTSA (2015). New Car Assessment Program. Docket No. NHTSA-2015-0119.

NHTSA Final economic assessment, FMVSS No. 201, upper interior head protection, NHTSA docket 1996-1762-0003.

Nusholtz, G.S., Lux, P., Kaiker, P., \& Janicki, M.A. (1984). Head impact response—Skull deformation and angular accelerations (No. 841657). SAE Technical Paper.

Ommaya, A.K. (1984). The head: kinematics and brain injury mechanisms. The Biomechanics of Impact Trauma, 117-125.

Ommaya, A.K., \& Gennarelli, T.A. (1974). Cerebral concussion and traumatic unconsciousness. Correlation of experimental and clinical observations of blunt head injuries. Brain, 97(4), 633-654.

Ommaya, A.K., \& Hirsch, A.E. (1971). Tolerances for cerebral concussion from head impact and whiplash in primates. Journal of Biomechanics, 4(1), 13-21.

Ommaya, A.K., Rockoff, S.D., Baldwin, M., \& Friauf, W.S. (1964). Experimental concussion: a first report. Journal of Neurosurgery, 21(4), 249-265.

Ommaya, A.K., Hirsch, A.E., Yarnell, P., \& Harris, E.H. (1967). Scaling of experimental data on cerebral concussion in sub-human primates to concussion threshold for man (No. 670906). SAE Technical Paper. 
Ono, K., Kikuchi, A., Nakamura, M., Kobayashi, H., \& Nakamura, N. (1980). Human head tolerance to sagittal impact reliable estimation deduced from experimental head injury using subhuman primates and human cadaver skulls (No. 801303). SAE Technical Paper.

Padgaonkar, A.J., Krieger, K.W., \& King, A.I. (1975). Measurement of angular acceleration of a rigid body using linear accelerometers. Journal of Applied Mechanics, 42(3): 552-556.

Panzer, M.B., Myers, B.S., Capehart, B.P., \& Bass, C.R. (2012). Development of a finite element model for blast brain injury and the effects of CSF cavitation. Annals of Biomedical Engineering, 40(7), 1530-1544.

Panzer, M.B., Wood, G.W., \& Bass, C.R. (2014). Scaling in neurotrauma: how do we apply animal experiments to people? Experimental Neurology, 261, 120-126.

Pare, A. (1579), Oeuvres complets. Ed. by J. F. Malgaigne, Paris, 1840, 2, 23.

Pascrell, C.B. (2009). Introduction to the Report of the International Conference on Behavioral Health and Traumatic Brain Injury. The Clinical Neuropsy., 23(8), 1281-1290.

Patrick, L.M., Lissner, H.R., \& Gurdjian, E.S. (1963). Survival by design: Head protection. In Proceedings: American Association for Automotive Medicine Annual Conference (Vol. 7, pp. 483-499). Association for the Advancement of Automotive Medicine.

Payne, P.R. (1962). An analog computer which determines human tolerance to acceleration. In Impact Acceleration Stress. National Academy of Sciences-National Research Council, Washington.

Prange, M.T., \& Margulies, S.S. (2002). Regional, directional, and age-dependent properties of the brain undergoing large deformation. Journal of Biomechanical Engineering, 124(2), 244-252.

Prasad, P., Dalmotas, D., \& German, A. (2014). The field relevance of NHTSA's oblique research moving deformable barrier tests. Stapp Car Crash Journal, 58, 175-195.

Pudenz, R.H., \& Shelden, C.H. (1946). The Lucite Calvarium-A Method for Direct Observation of the Brain: II. Cranial trauma and brain movement. Journal of Neurosurgery, $3(6), 487-505$.

Rao, S.S., \& Yap, F.F. (1995). Mechanical vibrations (Vol. 4). Reading: Addison-Wesley.

Resch, J.E., Rach, A., Walton, S., \& Broshek, D.K. (2017). Sport Concussion and the Female Athlete. Clinics in sports medicine, 36(4), 717-739.

Robbins, D.H. (1983). Anthropometric specifications for mid sized male dummy (Vol. 2). No. UMTRI-83-53-2. 
Rowson, S., \& Duma, S.M. (2011). Development of the STAR evaluation system for football helmets: integrating player head impact exposure and risk of concussion. Annals of Biomedical Engineering, 39(8), 2130-2140.

Rowson, S., \& Duma, S.M. (2013). Brain injury prediction: Assessing the combined probability of concussion using linear and rotational head acceleration. Annals of Biomedical Engineering, 41(5), 873-882.

Rowson, S., Duma, S.M., Beckwith, J.G., Chu, J.J., Greenwald, R.M., Crisco, J.J., Brolinson, P.G., Duhaime, A.-C., McAllister, T.W., \& Maerlender, A.C. (2012). Rotational head kinematics in football impacts: An injury risk function for concussion. Annals of Biomedical Engineering, 40(1), 1-13.

Sack, I., Beierbach, B., Wuerfel, J., Klatt, D., Hamhaber, U., Papazoglou, S., Martus, P., $\&$ Braun, J. (2009). The impact of aging and gender on brain viscoelasticity. Neuroimage, $46(3), 652-657$.

SAE. J211/1: Instrumentation for impact test part electronic instrumentation. Technical Report, SAE International, 1995.

Sanchez, E.J., Gabler, L.F., McGhee, J.S., Olszko, A.V., Chancey, V.C., Crandall, J., \& Panzer, M.B. (2017). Evaluation of head and brain injury risk functions using sub-injurious human volunteer data. Journal of Neurotrauma, (ja).

Santiago, L.A., Oh, B.C., Dash, P.K., Holcomb, J.B., \& Wade, C.E. (2012). A clinical comparison of penetrating and blunt traumatic brain injuries. Brain Inj, 26(2), 107-125.

Sato, K., Taki, Y., Fukuda, H., \& Kawashima, R. (2003). Neuroanatomical database of normal Japanese brains. Neural Networks, 16(9), 1301-1310.

Saunders, J., Parent, D., \& Ames, E. (2015). NHTSA oblique crash test results: vehicle performance and occupant injury risk assessment in vehicles with small overlap countermeasures. In $24^{\text {th }}$ International Conference on the Enhanced Safety of Vehicles.

Ellenbogen, R.G., Abdulrauf, S.I., \& Sekhar, L.N. (2012). Principles of Neurological Surgery E-Book: Expert Consult-Online. Elsevier Health Sciences.

Slattenschek, A., \& Tauffkirchen, W. (1970). Critical evaluation of assessment methods for head impact applied in appraisal of brain injury hazard, in particular in head impact on windshields (No. 700426). SAE Technical Paper.

Smith, D.H., Meaney, D.F., \& Shull, W.H. (2003). Diffuse axonal injury in head trauma. The Journal of Head Trauma Rehabilitation, 18(4), 307-316.

Sowell, E.R., Peterson, B.S., Thompson, P.M., Welcome, S.E., Henkenius, A.L., \& Toga, A.W. (2003). Mapping cortical change across the human life span. Natural Neuroscience, 6(3), 309-315. 
Stalnaker, R.L., Fogle, J.L., \& McElhaney, J.H. (1971). Driving point impedance characteristics of the head. Journal of Biomechanics, 4(2), 127-139.

Stalnaker, R.L., Alem, N.M., \& Benson, J.B. (1977). Validation studies for head impact injury model. No. DOT-HS-802566 Final Report.

Subit, D., Kerrigan, J., Crandall, J.R., Fukuyama, K., Yamazaki, K., Kamiji, K., \& Yasuki, T. (2008). Pedestrian-vehicle interaction: kinematics and injury analysis of four full scale tests. In Proceedings of IRCOBI Conference.

Sullivan, S., Eucker, S.A., Gabrieli, D., Bradfield, C., Coats, B., Maltese, M.R., Lee, J., Smith, C., \& Margulies, S.S. (2015). White matter tract-oriented deformation predicts traumatic axonal brain injury and reveals rotational direction-specific vulnerabilities. Biomechanics and Modeling in Mechanobiology, 14(4), 877-896.

Takahashi, Y., \& Yanaoka, T. (2017). A study of injury criteria for brain injuries in traffic accidents. In $25^{\text {th }}$ International Technical Conference on the Enhanced Safety of Vehicles.

Takhounts, E.G., Crandall, J.R., \& Darvish, K. (2003a). On the importance of nonlinearity of brain tissue under large deformations. Stapp Car Crash Journal, 47, 79-92.

Takhounts, E.G., Eppinger, R.H., Campbell, J.Q., Tannous, R.E., \& others (2003b). On the development of the SIMon finite element head model. Stapp Car Crash J., 47, 107.

Takhounts, E.G., Ridella, S.A., Hasija, V., Tannous, R.E., Campbell, J.Q., Malone, D., Danelson, K., Stitzel, J., Rowson, S., \& Duma, S. (2008). Investigation of traumatic brain injuries using the next generation of simulated injury monitor (SIMon) finite element head model. Stapp Car Crash Journal, 52, 1.

Takhounts, E.G., Hasija, V., \& Eppinger, R.H. (2009). Analysis of 3D rigid body motion using the nine accelerometer array and the randomoly distributed in-plane accelerometer systems. In $21^{\text {st }}$ International Technical Conference on the Enhanced Safety of Vehicles.

Takhounts, E.G., Hasija, V., Ridella, S.A., Rowson, S., \& Duma, S.M. (2011). Kinematic rotational brain injury criterion (BRIC). In $22^{\text {nd }}$ International Technical Conference on the Enhanced Safety of Vehicles.

Takhounts, E.G., Craig, M.J., Moorhouse, K., McFadden, J., \& Hasija, V. (2013). Development of brain injury criteria (BrIC). Stapp Car Crash Journal, 57, 243-266.

Tanielian, T., Haycox, L.H., Schell, T.L., Marshall, G.N., Burnam, M.A., Eibner, C., Karney, B.R., Meredith, L.S., Ringel, J.S., \& Vaiana, M.E. (2008). Invisible wounds of war. Summary and recommendations for addressing psychological and cognitive injuries. Rand Corp Santa Monica, CA.

Taylor, C.A. (2017). Traumatic brain injury-related emergency department visits, hospitalizations, and deaths — United States, 2007 and 2013. MMWR. Surveillance Summaries, 66. 
Teasdale, G., \& Jennett, B. (1974). Assessment of coma and impaired consciousness: a practical scale. The Lancet, 304(7872), 81-84.

Thibault, K.L., \& Margulies, S.S. (1998). Age-dependent material properties of the porcine cerebrum: effect on pediatric inertial head injury criteria. Journal of Biomechanics, 31(12), 1119-1126.

Thibault, L.E., Gennarelli, T.A., Margulies, S.S., Marcus, J., \& Eppinger, R. (1990). The strain dependent pathophysiological consequences of inertial loading on central nervous system tissue. In Proceedings of IRCOBI Conference.

Thornton, S., \& Marion, J. (2004). Classical Mechanics of Particles and Systems (5 ${ }^{\text {th }}$ ed).

Trosseille, X., Tarriere, C., Lavaste, F., Guillon, F., \& Domont, A. (1992). Development of a FEM of the human head according to a specific test protocol (No. 922527). SAE Technical Paper.

Versace, J. (1971). A Review of the severity index (No. 710881). SAE Technical Paper.

Viano, D.C., Withnall, C., \& Halstead, D. (2012). Impact performance of modern football helmets. Annals of Biomedical Engineering, 40(1), 160-174.

Wu, L.C., Nangia, V., Bui, K., Hammoor, B., Kurt, M., Hernandez, F., Kuo, C., \& Camarillo, D.B. (2016). In vivo evaluation of wearable head impact sensors. Annals of Biomedical Engineering, 44(4), 1234-1245.

Yanaoka, T., Dokko, Y., \& Takahashi, Y. (2015). Investigation on an Injury Criterion Related to Traumatic Brain Injury Primarily Induced by Head Rotation (No. 2015-011439). SAE Technical Paper.

Yoganandan, N., Arun, M.W., \& Pintar, F.A. (2014). Normalizing and scaling of data to derive human response corridors from impact tests. J. Biomechanics, 47(8), 1749-1756.

Zhang, L., Yang, K.H., \& King, A.I. (2004). A Proposed Injury Threshold for Mild Traumatic Brain Injury. Journal of Biomechanical Engineering, 126(2), 226-236.

Zhao, W., \& Ji, S. (2016a). Real-time, whole-brain, temporally resolved pressure responses in translational head impact. Interface Focus, 6(1), 20150091.

Zhao, W., \& Ji, S. (2016b). Brain strain uncertainty due to shape variation in and simplification of head angular velocity profiles. Biomechanics and Modeling in Mechanobiology, 16(2), 449-461.

Zhao, W., Cai, Y., Li, Z., \& Ji, S. (2017). Injury prediction and vulnerability assessment using strain and susceptibility measures of the deep white matter. Biomechanics and Modeling in Mechanobiology, 1-19. 


\section{List of Published Work and Contribution}

Paper I: Gabler, L.F., Crandall, J.R., \& Panzer, M.B. (2016) Investigating Brain Injury Tolerance in the Sagittal Plane Using a Finite Element Model of the Human Head. International Journal of Automotive Engineering, 7(1), 37-43.

Division of work between authors: All authors contributed to the design of the study. The study was performed by Gabler. The paper was written by Gabler and edited by Panzer. Panzer and Crandall provided oversight and scientific guidance.

Paper II: Gabler, L.F., Crandall, J.R., \& Panzer, M.B. (2016) Assessment of Kinematic Brain Injury Metrics for Predicting Strain Responses in Diverse Automotive Impact Conditions. Annals of Biomedical Engineering, 44(12), 3705-3718. DOI: 10.1007/s10439016-1697-0.

Division of work between authors: Gabler designed the study under the supervision of Panzer. Collection and processing of head kinematics, injury criteria literature review and calculation, FE model simulations, and analysis were performed by Gabler. The paper was written by Gabler and edited by Panzer. Crandall provided scientific guidance.

Paper III: Gabler LF, Joodaki H, Crandall JR, \& Panzer MB (2016) Development of a Single-Degree-of-Freedom Mechanical Model for Predicting Strain-based Brain Injury Responses. Journal of Biomechanical Engineering, DOI:10.1115/1.4038357.

Division of work between authors: Gabler designed the study under the supervision of Panzer. Analytical and FE modeling, analysis and paper writing was performed by Gabler. The paper was edited by Panzer and Joodaki. Crandall provided scientific guidance.

Paper IV: Gabler, L.F., Crandall. J.R., \& Panzer, M.B. (2016) Development of an Injury Metric for Predicting Brain Strain Responses using Head Kinematics. Annals of Biomedical Engineering. (in review)

Division of work between authors: Gabler designed the study under the supervision of Panzer. Gabler developed the UBrIC metric and performed all model fits and analysis. The paper was written by Gabler and edited by Panzer. Crandall provided scientific guidance.

\section{Other related publications (non-journal)}

Paper V: Gabler, L.F., Crandall, J.R., \& Panzer, M.B. (2014) On the Application of BrIC to the Biomechanics of Various Automotive Impact Scenarios. 42 ${ }^{\text {nd }}$ International Workshop on Human Subjects for Biomechanical Research, San Diego, CA. (paper) 
Paper VI: Panzer, M.B., Gabler, L.F., Rodenberger, E.J., Joodaki, H., \& Crandall, J.R. (2016). Assessment of Brain Injury Criterion (BrIC). In response to the National Highway Traffic Safety Administration's Request for public comment on the New Car Assessment Program [Docket No. NHTSA-2015-0119] at: www.regulations.gov. (public comment)

Paper VII: Gabler, L.F., Joodaki, H., Crandall, J.R., \& Panzer, M.B. (2016) Toward Development of a Single-Degree-of-Freedom Mechanical Model for Predicting Brain Injury. In Proceedings of IRCOBI Conference (Asia), Seoul, South Korea. (abstract)

Paper VIII: Gabler, L.F., Rodenberger, E.J., Crandall, J.R., \& Panzer, M.B. (2016) Predicting Brain Injury Using Head Kinematics. National Neurotrauma Symposium, Lexington, KY. (poster)

Paper IX: Gabler, L.F., Crandall, J.R., Panzer, M.B., Praxl, N., \& Wernicke, P. (2017) Development of Improved Brain Injury Predictors for Diverse Impacts. $61^{\text {st }}$ Stapp Car Crash Conference, Charleston, SC. (short communication) 